\begin{abstract}
Title of Dissertation: $\quad$ DYNAMIC DECISION MAKING FOR LESS-THAN-TRUCKLOAD TRUCKING OPERATIONS

Behrang Hejazi, Doctor of Philosophy, 2009

Directed By: $\quad$ Professor Ali Haghani

Department of Civil and Environmental Engineering
\end{abstract}

On a typical day, more than 53 million tons of goods valued at about $\$ 36$ million are moved on the US multimodal transportation network. An efficient freight transportation industry is the key in facilitating the required movement of raw materials and finished products. Among different modes of transportation, trucking remains the shipping choice for many businesses and is increasing its market share. Less-than-truckload (LTL) trucking companies provide a transportation service in which several customers are served simultaneously by using the same truck and shipments need to be consolidated at some terminals to build economical loads.

Intelligent transportation system (ITS) technologies increase the flow of available data, and offer opportunities to control the transportation operations in realtime. Some research efforts have considered real-time acceptance/rejection of shipping requests, but they are mostly focused on truckload trucking operations. This study tries to use real-time information in decision making for LTL carriers in a dynamically changing environment.

The dissertation begins with an introduction of LTL trucking operations and different levels of planning for this type of motor carriers, followed by the review of 
literature that are related to tactical and operational planning. Following a brief discussion on multi commodity network flow problems and their solution algorithm, a mathematical model is proposed to deal with the combined shipment and routing problem.

Furthermore, a decision making procedure as well as a decision support application are developed and are presented in this dissertation. The main step in the decision making procedure is to solve the proposed mathematical problem. Three heuristic solution algorithms are proposed and the quality of the solutions is evaluated using a set of benchmark solutions.

Three levels of numerical experiments are conducted considering an auto carrier that operates on a hub-and-spoke network. The accuracy of the mathematical model and the behavior of the system under different demand/supply situations are examined. Also, the performance of the solutions provided by the proposed heuristic algorithms is compared and the best solution method is selected. The study suggests that significant reductions in operational costs are expected as the result of using the proposed decision making procedure. 


\section{DYNAMIC DECISION MAKING \\ FOR LESS-THAN-TRUCKLOAD TRUCKING OPERATIONS}

By

Behrang Hejazi

Dissertation submitted to the Faculty of the Graduate School of the University of Maryland, College Park, in partial fulfillment of the requirements for the degree of

Doctor of Philosophy

2009

Advisory Committee:

Professor Ali Haghani, Supervisor

Professor Paul M. Schonfeld

Professor Gang-Len Chang

Professor Martin Dresner

Professor Philip T. Evers 
CCopyright by

Behrang Hejazi

2009 


\section{Acknowledgments}

I would like to express my gratitude to my advisor, Prof. Ali Haghani who offered invaluable assistance, support and guidance. Special thanks to the members of the supervisory committee, Prof. Paul Schonfeld, Prof. Hani Mahmassani, Prof. Gang-Len Chang, Prof. Martin Dresner and Prof. Philip Evers for all their support during this research project. I would also like to express my thanks to my wife, my parents and my brother for the support they provided me. 


\section{Table of Contents}

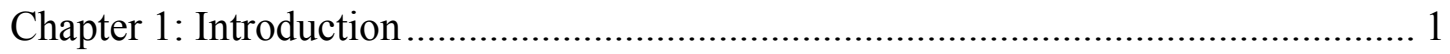

Freight Transportation ............................................................................... 1

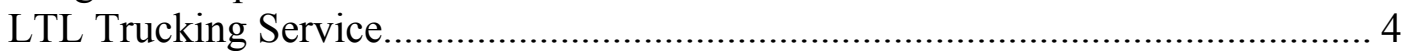

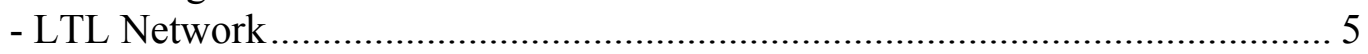

- Line Operations Network ……………………………………………….... 5

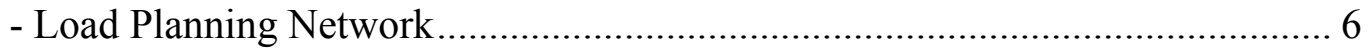

- An Illustration of LTL Trucking Operations.................................................... 6

Market Segmentation ................................................................................. 10

- Long-haul Carriers...................................................................................... 10

- Superregional Carriers .............................................................................. 10

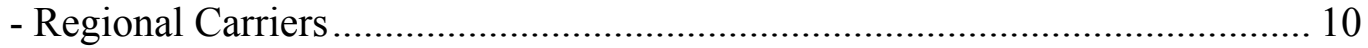

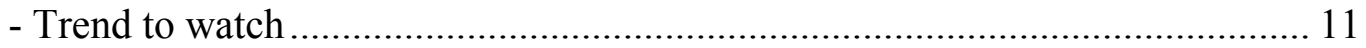

- New Technologies and LTL Operations …………………........................... 12

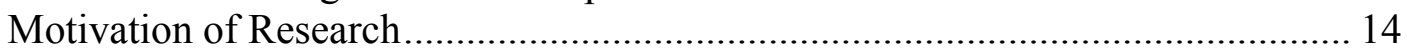

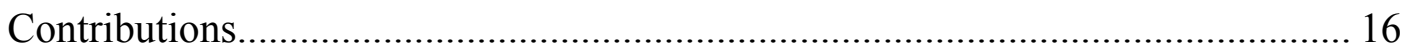

Organization of the Dissertation ...................................................................... 17

Chapter 2: Literature Review ............................................................................... 19

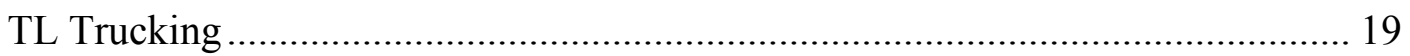

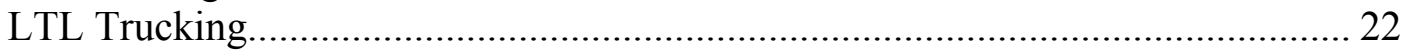

- Strategic (log-term) Planning ...................................................................... 23

- Tactical (medium-term) Planning ..................................................................... 23

- Operational (short-term) Planning................................................................ 23

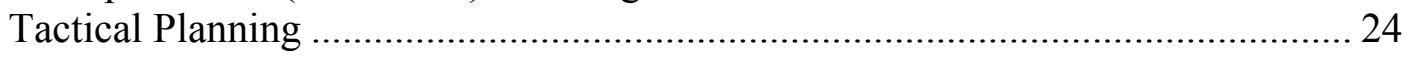

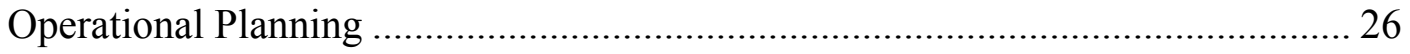

MCNF problem and solution algorithms ………………............................. 28

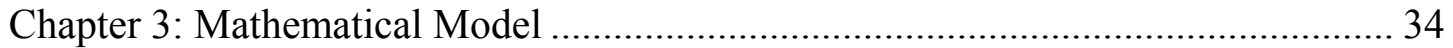

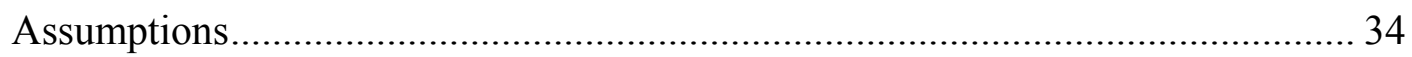

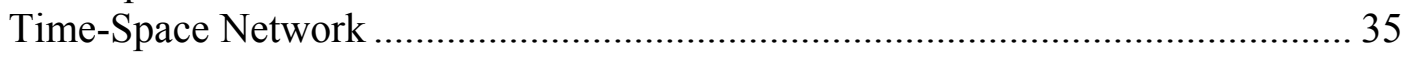

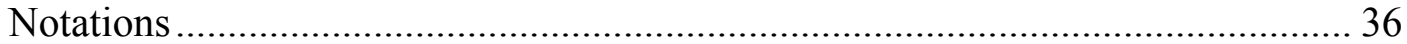

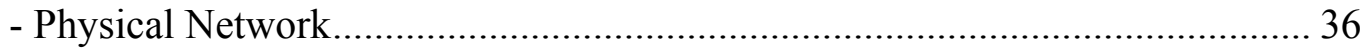

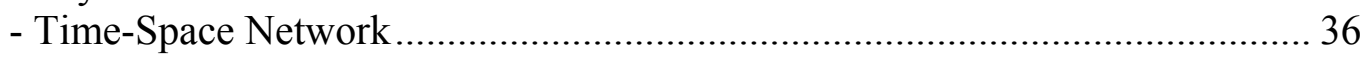

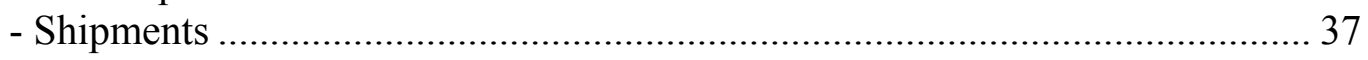

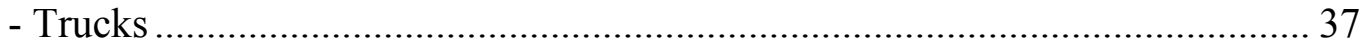

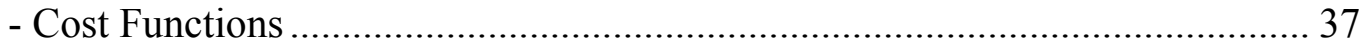

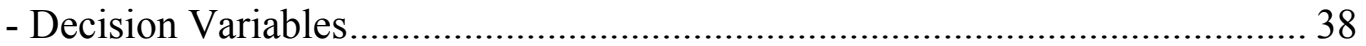

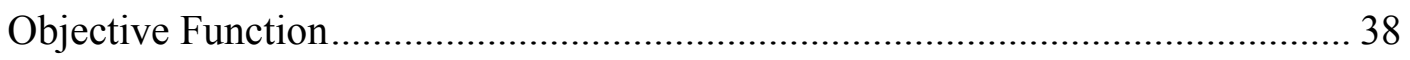

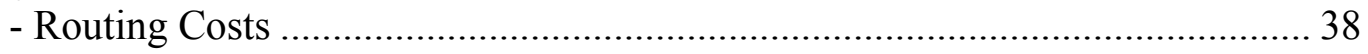

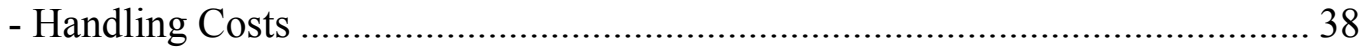

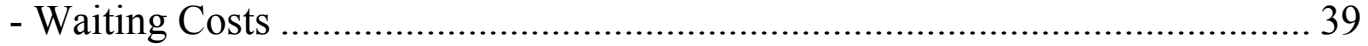

- No-Delivery Penalty Costs ........................................................................... 39

- Late-Delivery Penalty Costs ............................................................................ 39

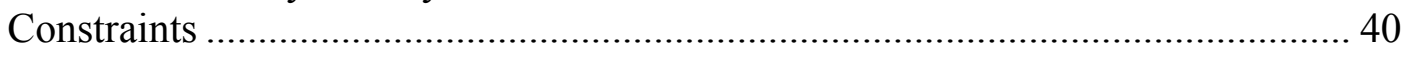

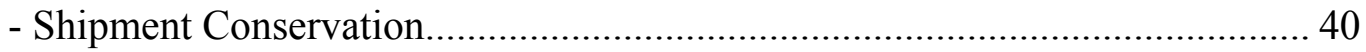

- Shipment-Loaded Truck Connection: .............................................................. 42 


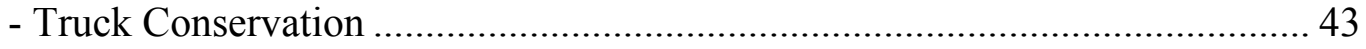

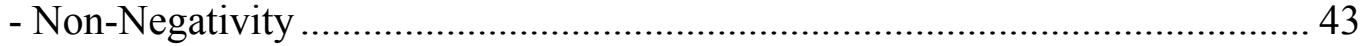

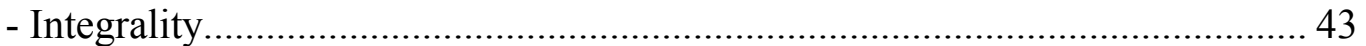

Mathematical Formulation............................................................................ 44

Extension 1: Non-homogeneous Fleet.............................................................. 45

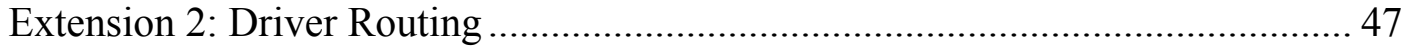

Extension 3: Early Delivery Costs ..................................................................... 50

Chapter 4: Decision Making Procedures ………………......................................... 52

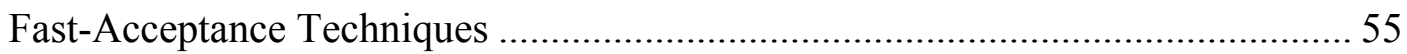

- Technique 1 (Fixed Truck Routing) ………………………………………..... 55

- Technique 2 (Adaptive Truck Routing).......................................................... 56

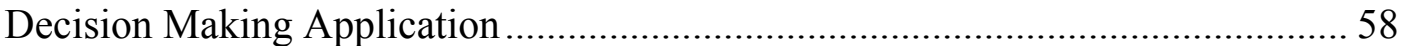

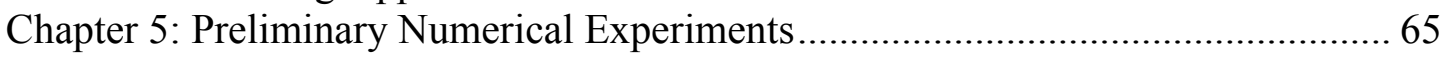

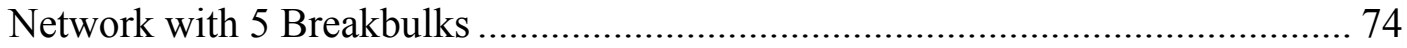

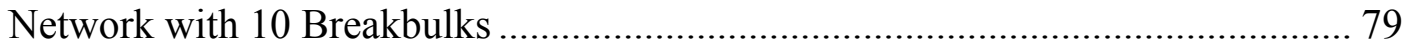

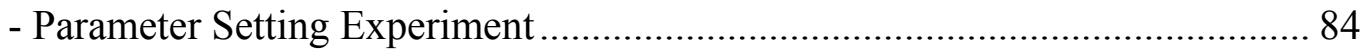

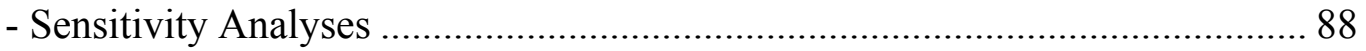

- Driver Routing ……………………………………………………. 116

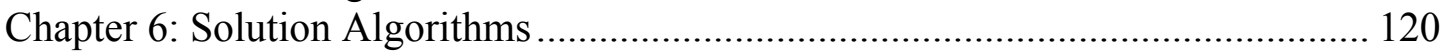

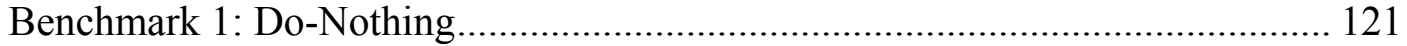

Benchmark 2: Literature-Reported Operation ..................................................... 121

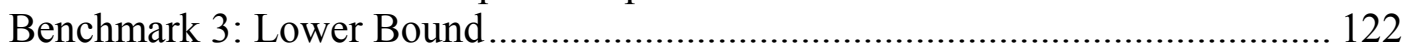

Algorithm 1: Path-Based Heuristic Search ...................................................... 125

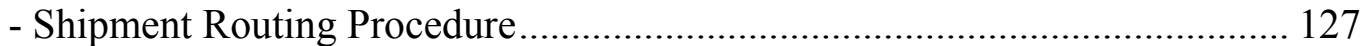

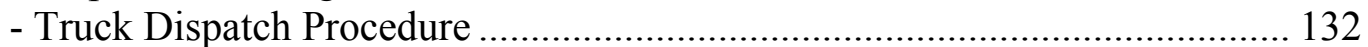

- Objective Function Calculation .................................................................... 132

- Solution Improvements................................................................................. 133

Algorithm 2: Path-Based Heuristic Search with Simulated Annealing ................. 134

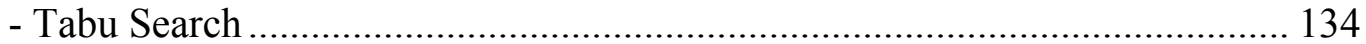

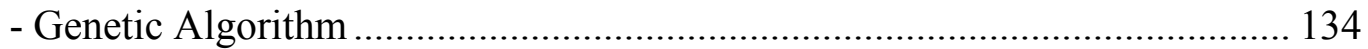

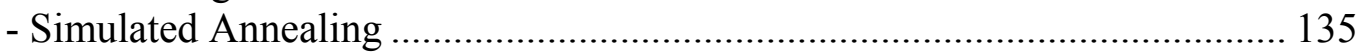

Algorithm 3: Path-Based Network Partitioning .................................................. 140

Chapter 7: Numerical Experiments.................................................................... 149

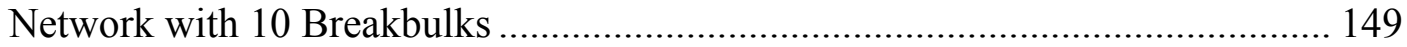

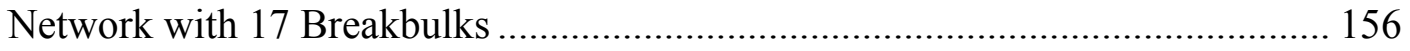

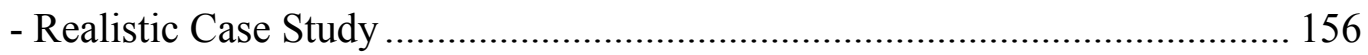

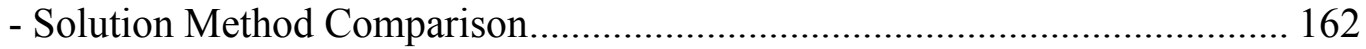

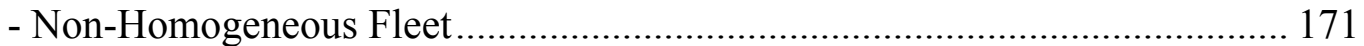

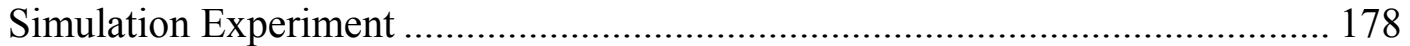

Chapter 8: Summary, Conclusions and Recommendations for Future Research .... 188

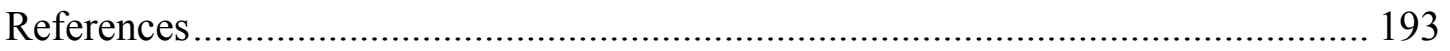




\section{Chapter 1: Introduction}

\section{Freight Transportation}

According to an estimate by the Bureau of Transportation Statistics (BTS) of the US DOT's Research and Innovative Technology Administration (RITA) and FHWA, over 19 billion tons of freight valued at $\$ 13$ trillion was transported in the United States in 2002. This means that on a typical day about 53 million tons of goods valued at about $\$ 36$ million moved on the US multimodal transportation network [1].

An efficient freight transportation industry is the key in facilitating the required movement of raw materials and finished goods. Maintaining the availability of raw materials and providing fast and reliable delivery of final product, support production, trade, and consumption activities. Freight transportation is a major element of the economy and needs to adapt to the current rapidly changing economic trends such as just-in-time production and distribution, internet-based electronic businesses (e-commerce), business-to-business, and business-to-customers environments where distributors and retailers are being eliminated.

Carriers provide transportation services. Railways, shipping lines, intermodal containers, and trucking companies are different types of carriers. Despite the recent advances in the US freight transportation system, some previous freight trends continue. Among the modes of transportation, trucking industry's market share is increasing and it is still the best choice for many businesses. According to [2], in 2002, trucking industry's share was estimated to be 74 percent of the total value, 67 percent of the weight, and 40 percent of the overall ton-miles (Table 1). In 2002, the total ton-miles for this mode of freight transportation had increased more than $40 \%$ compared to1993. Since 1980, overall truck vehicle miles have doubled from 108 billion to 216 billion in 2003. Figure 1 shows the estimated average annual daily truck traffic for 1998 and 2020 [1]. 
Table 1 - Commercial freight activity in the U.S. by mode of transportation [2]

\begin{tabular}{|c|c|c|c|c|c|c|c|c|c|}
\hline Mode of Transportation & $\begin{array}{c}\text { Value } \\
1993 \\
\text { (billion \$) }\end{array}$ & $\begin{array}{c}\text { Value } \\
2002 \\
\text { (billion \$) }\end{array}$ & $\begin{array}{c}\text { Value } \\
\text { Change } \\
(\%)\end{array}$ & $\begin{array}{c}\text { Tons } \\
1993 \\
\text { (millions) }\end{array}$ & $\begin{array}{c}\text { Tons } \\
\mathbf{2 0 0 2} \\
\text { (millions) }\end{array}$ & $\begin{array}{c}\text { Tons } \\
\text { Change } \\
(\%)\end{array}$ & $\begin{array}{c}\text { Ton-miles } \\
1993 \\
\text { (billions) }\end{array}$ & $\begin{array}{c}\text { Ton-miles } \\
2002 \\
\text { (billions) }\end{array}$ & $\begin{array}{c}\text { Ton-miles } \\
\text { Change } \\
(\%)\end{array}$ \\
\hline All Modes & $5,846.3$ & $8,397.2$ & 43.6 & $9,688.5$ & $11,667.9$ & 20.4 & $2,420.9$ & $3,137.9$ & 29.6 \\
\hline Total Single Modes & $4,941.5$ & $7,049.4$ & 42.7 & $8,922.3$ & $11,086.7$ & 24.3 & $2,136.9$ & $2,867.9$ & 34.2 \\
\hline Truck & $4,403.5$ & $6,235.0$ & 41.6 & $6,385.9$ & $7,842.8$ & 22.8 & 869.5 & $1,255.9$ & 44.4 \\
\hline For-hire truck & $2,625.1$ & $3,757.1$ & 43.1 & $2,808.3$ & $3,657.3$ & 30.2 & 629.0 & 959.6 & 52.6 \\
\hline Private truck & $1,755.8$ & $2,445.3$ & 39.3 & $3,543.5$ & $4,149.7$ & 17.1 & 235.9 & 291.1 & 23.4 \\
\hline Rail & 247.4 & 310.9 & 25.7 & $1,544.1$ & $1,873.9$ & 21.4 & 942.6 & $1,261.6$ & 33.8 \\
\hline Water & 61.6 & 89.3 & 45.0 & 505.4 & 681.2 & 34.8 & 272.0 & 282.6 & 3.9 \\
\hline Shallow draft & 40.7 & 57.5 & 41.2 & 362.5 & 458.6 & 26.5 & 164.4 & 211.5 & 28.7 \\
\hline Great Lakes & NA & 0.8 & NA & 33.0 & 38.0 & 15.1 & 12.4 & 13.8 & 11.4 \\
\hline Deep draft & 19.7 & 31.0 & 57.1 & 109.9 & 184.6 & 67.9 & 95.2 & 57.4 & -39.8 \\
\hline Air & 139.1 & 265.0 & 90.5 & 3.1 & 3.8 & 19.8 & 4.0 & 5.8 & 45.5 \\
\hline Pipeline & 89.8 & 149.2 & 66.1 & 483.6 & 685.0 & 41.6 & NA & NA & NA \\
\hline Total Multiple Modes & 662.6 & $1,079.2$ & 67.7 & 225.7 & 216.7 & -4.0 & 191.5 & 225.7 & 17.9 \\
\hline $\begin{array}{l}\text { Parcel, U.S. Postal } \\
\text { Service or courier }\end{array}$ & 563.3 & 987.7 & 75.4 & 18.9 & 25.5 & 35.0 & 13.2 & 19.0 & 44.5 \\
\hline Truck and rail & 83.1 & 69.9 & -15.8 & 40.6 & 43 & 5.8 & 37.7 & 45.5 & 20.8 \\
\hline Truck and water & 9.4 & 14.4 & 52.9 & 68.0 & 23.3 & -65.7 & 40.6 & 32.4 & -20.2 \\
\hline Rail and water & 3.6 & 3.3 & -8.4 & 79.2 & 105 & 32.7 & 70.2 & 115.0 & 63.8 \\
\hline Other multiple modes & 3.2 & 3.8 & 18.8 & 18.9 & 19.8 & 4.4 & NA & 13.8 & NA \\
\hline Total Unknown Modes & 242.3 & 268.6 & 10.9 & 540.5 & 364.6 & -32.6 & 92.6 & 44.2 & -52.2 \\
\hline
\end{tabular}




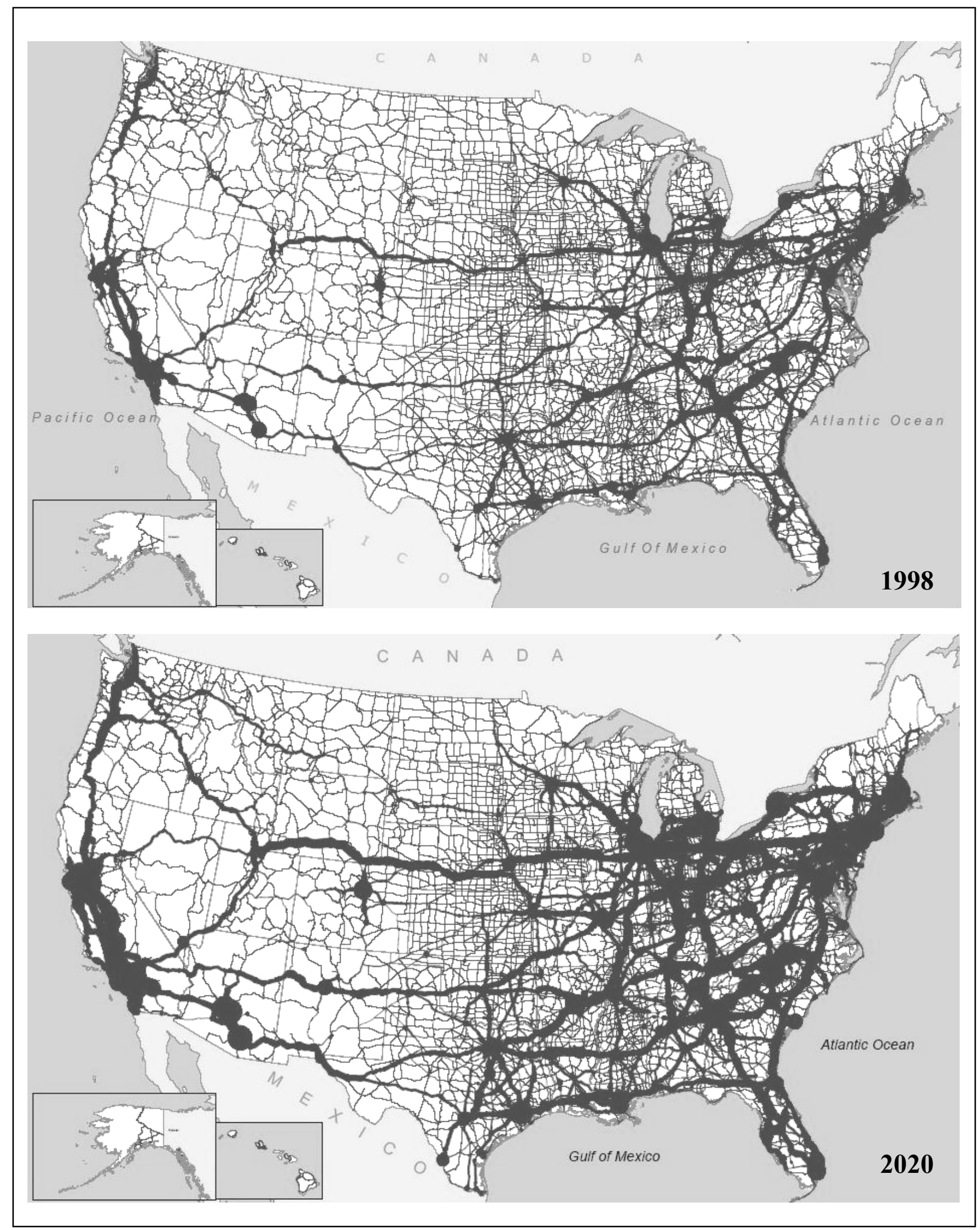

Figure 1 - Estimated average annual daily truck traffic for 1998, and 2020 
From an operational point of view, trucking services are classified as "truckload" (TL), and "less-than-truckload" (LTL). Truckload trucking offers a typical example of door-to-door transportation, where a truck is assigned to each customer. When a customer request for pickup and delivery of a load, the carrier decides whether to accept or reject the request. If the carrier accepts the load, a truck moves empty to its origin to pick it up and move it to its destination. After unloading, the truck is ready for a new assignment. The carrier may assign a new load to the truck, move the truck empty to a new location to handle future demands, or keep it idle at the same location. Unlike truckload operations, in less-than-truckload trucking several customers are served simultaneously by using the same truck. The main focus of this study is LTL trucking services, which will be discussed in the following sections.

\section{$\underline{\text { LTL Trucking Service }}$}

Considering a trucking company with a fleet of trucks, there is usually a sequence of delivery requests within a region either by phone, fax, or Internet. A pickup-delivery (TL) trucking service moves the loads to the origin terminal. Through LTL network, shipments are moved to the destination terminal using highway trailers (long-haul movement). At the end, loads are delivered to the final destination, again using TL trucking services. LTL motor carriers haul shipments weighing from 100 to 10,000 pounds that are less than a full truckload, and the majority of them are less than 1000 pounds. Since trailers hold 30,000 to 50,000 pounds, a truck can carry an average of 20 to 30 shipments that may have different origins and destinations. Thus,

shipments need to be consolidated at some terminals to build economical loads and that is why this operation is called "consolidation transportation". 


\section{- LTL Network}

An LTL network consists of two types of terminals (Figure 2):

- End-of-line: It is the origin and destination of freights. Note that, the local pickup and delivery problem is not solved in this study. Hence, for each endof-line terminal the closest hub is considered as the origin/destination of the loads.

- Breakbulk (hub): It handles the unloading, sorting, and reloading of the freight from one truck to another. Each end-of-line is typically associated with one "primary break" which is usually the closest breakbulk with respect to the direction of the delivery. The end-of-lines connected to a given primary breakbulk are called the "satellites" of that breakbulk.

\section{- Line Operations Network}

Because of the restrictions on driving time, the movement of trucks between terminals must follow a line operations network. Figure 3-1 shows an example. The length of any link in this network cannot exceed what a driver can cover in 11 hours driving time. The nodes of the line operations network are points at which drivers are often changed and consist of all terminals as well as additional relay points.

When the volume of shipment between two terminals is high, the carrier is said to offer a "direct service" between two terminals and as a rule, trucks are loaded at one terminal and completely unloaded at the other. Offering direct service between two terminals generally implies a regular operation with trailers leaving at least two or three times per week. At lower frequencies, some shipments may experience unacceptably long delays waiting for the trailer to leave. 
- Load Planning Network

It is important to determine to which pairs of terminals the carrier should offer direct service. Since a direct service between two terminals may comprise several links of the line operations network, the load-planning network is introduced. Figure 3-2 illustrates an example. In principle, the load-planning network might consist of links that connect every terminal to every other terminal. For large national networks, however, direct end-of-line to end-of-line movements are rare and for discussion purposes can be ignored. Thus, the set of load planning links can be viewed as all links that begin or end at a breakbulk. Of this total remaining set of load planning links, only about 10 to $20 \%$ will be used for direct service. The problem in strategic and tactical level of planning is to determine which ones to use.

Carriers generally manage level of service by requiring that the frequency of service over each link satisfy a specified minimum. In most cases, direct service will not be offered between two terminals unless it is possible to fill at least the minimum number of trailers per week, with the exception of movements between satellites and primary breaks where departures occur regardless of the amount of freight on the trailer. Typical weekly minimums for breakbulk to breakbulk moves or from an endof-line and non-primary break are 3-5 trailers per week.

- An Illustration of LTL Trucking Operations

Figure 4 illustrates an LTL trucking operations. The LTL network consists of 12 end-of-lines and 5 breakbulks (hubs). A total of 36 loads must be shipped to their destinations using a fleet of 11 trucks. The capacity of each truck is 3 loads. Different colors have been used to define the relationship between loads, and their final destinations. Each load must be shipped to the associated end-of-line with the similar color. As an example, it is shown how three "green" loads from different origins are shipped through their primary breaks to an intermediate hub, where the consolidation takes place, and then loads are delivered to the final destination. 


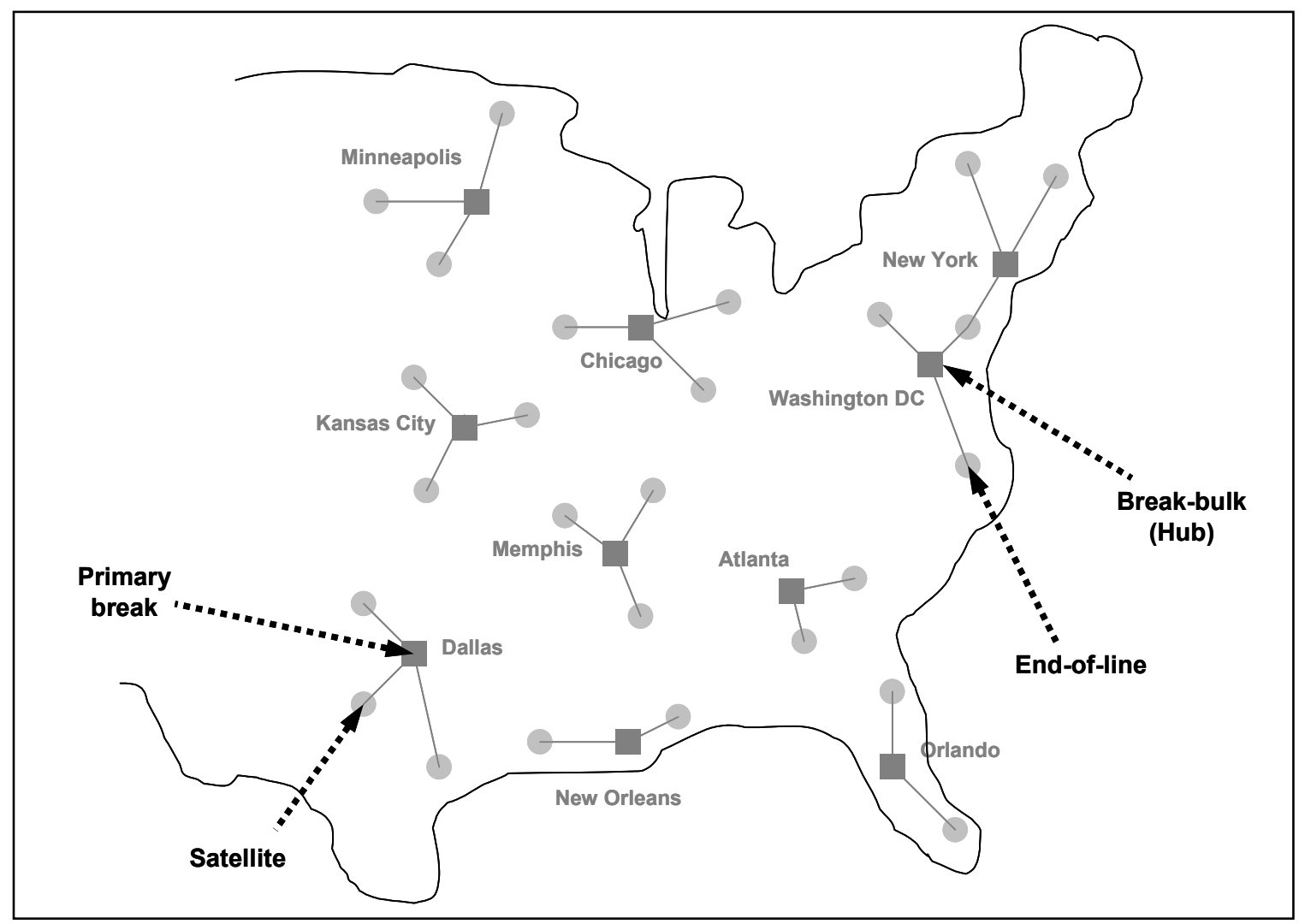

Figure 2 - Elements of LTL trucking network 

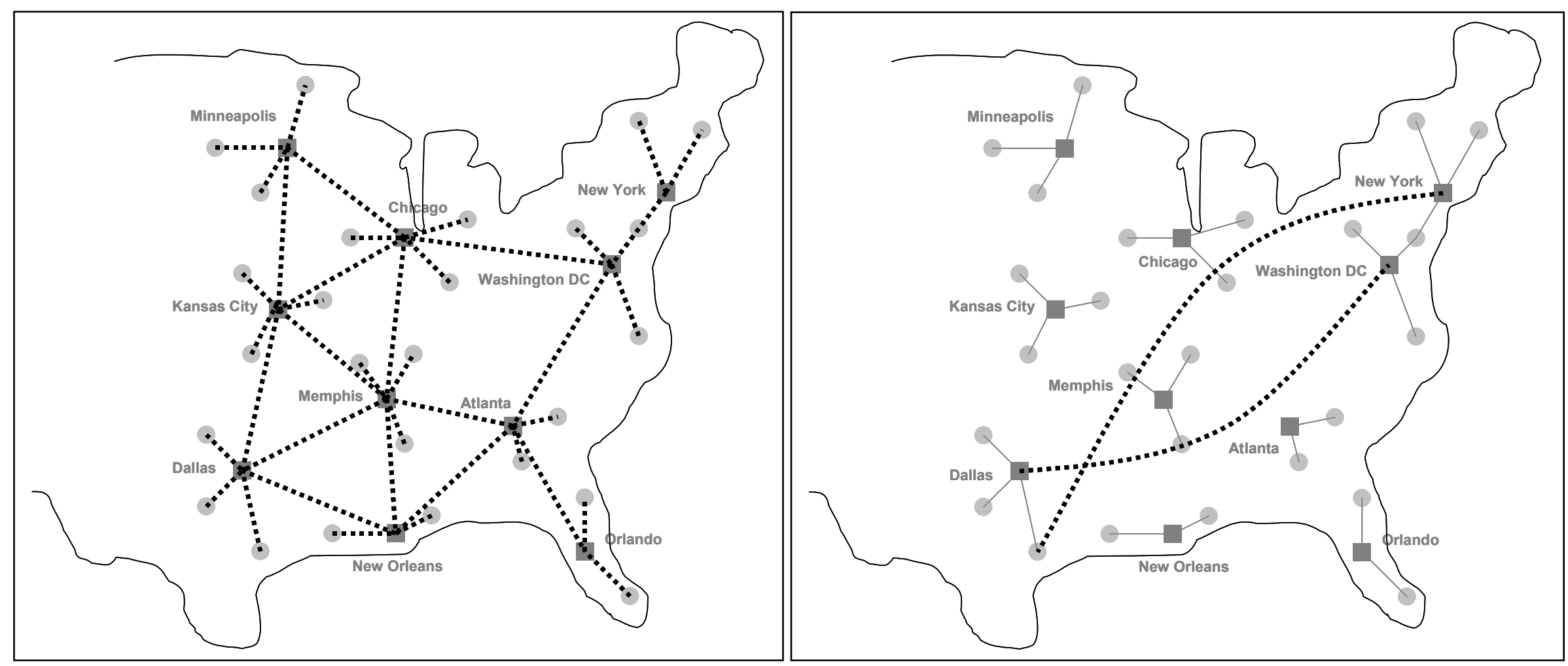

Figure 3 - Line Operation Network (1), and Load Planning Networks (2) 

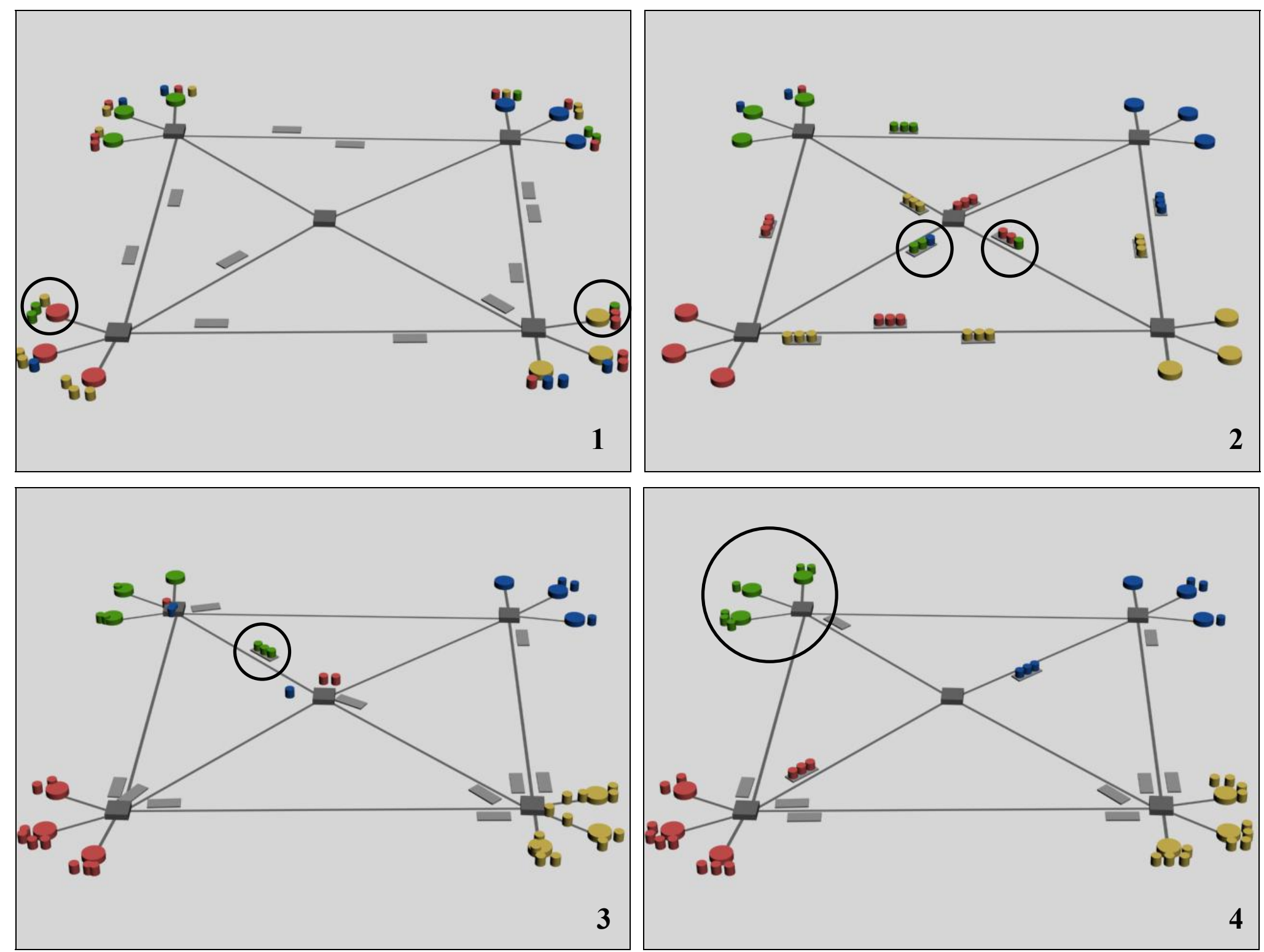

Figure 4 - An Illustration of LTL Trucking Operations (12 end-of-lines, 5 hubs, 36 loads, and 11 trucks) 


\section{$\underline{\text { Market Segmentation }}$}

The LTL market is often segmented between national and regional carriers for ease of discussion. However, this is a simplistic segmentation, and American Trucking Association Foundation (ATA) proposes a better segmentation [3]:

- Long-haul Carriers

The long haul carriers are usually referred to as national LTL carriers, and generally offer full coverage of the United States. Carriers in this long-haul group use union labor. Their average length of haul is approximately 1200 miles, and transit times run 3 to 5 days, with some 2-day and some 6-day lanes. National LTL carriers are operating extensive hub-and-spoke terminal networks, and generate approximately 38 percent of total LTL sector revenues.

\section{- Superregional Carriers}

These carriers account for roughly 30 percent of LTL sector revenues. Like the long-haul carriers, the superregionals operate hub-and-spoke networks. In contrast however, they focus mostly on 2-day lanes with significant overnight and 3-day lanes as well. Their average length of haul runs from 400 to 750 miles. Many private LTL carriers also qualify as superregionals.

\section{- Regional Carriers}

These carriers focus on overnight freight movements, with some specific 2day lanes. In contrast to carriers with hub-and-spoke networks, this group focuses on direct movements of freight between terminals with no intermediate sort at a hub. The average length of haul is generally less than 300 miles. Most regional carriers are nonunion. Regionals generate roughly 27 percent of LTL sector revenues. 
- Trend to watch

When gas price was still at a dollar a gallon, LTL carriers could afford a little more inefficiency. But with gas prices and other costs going up in recent years, errors in daily operations could hurt the profit of LTL trucking business significantly. YRC Worldwide Inc. and Old Dominion freight Line Inc., two of the US largest LTL carriers both reported 2007 third-quarter earnings that missed Wall Street's expectations [4]. FedEx freight, a leading regional next-day and second-day LTL carrier announced more than 5 percent rate increase in January 2008 [5].

In recent years, Superregional carriers have been expanding their service areas and as a result the long-haul LTL carriers have faced a loss of market share to superregional and regional LTL carriers [3]. The superregional carriers have been expanding their geographic coverage areas for two reasons:

- As shippers use fewer trucking companies it makes sense to offer a broader geography to retain the business of larger accounts. Their wider scope therefore puts superregional carriers in a better competitive position.

- It is easier for the superregional carriers to compete with long-haul carriers than with pure regional carriers. The long-haul carriers, with their labor forces and overbuilt terminal networks, are high cost carriers. The superregional carriers can often offer faster and/or cheaper transportation. Furthermore, the 1994 Teamster strike against the long-haul carriers encouraged shippers to deal with nonunion carriers to avoid service disruptions.

The national LTL carriers are working hard to meet the competitive challenges. Their strategy is to:

- $\quad$ Try to give the customers more of what they want, such as faster transit times

- Increase their market share with pricing

Carriers try to reduce the number of times the freight is handled. The goal is to cut transit times throughout the network by at least a day and to reduce handling and claims costs. The approach is slightly different from carrier to carrier, but most are closing a significant number of end-of-line terminals and consolidating the number of 
hubs in their systems. They are all moving to scheduled departures for their line-haul moves. The lower-cost superregional LTL carriers are likely to continue to gain market share from the unionized long-haul carriers in the 2- and 3-day lane markets, although it could be a long-term process.

- New Technologies and LTL Operations

As a part of this research study, a limited survey was conducted to investigate the type of technologies, in forms of hardware or software, which is being used by LTL carriers for fleet management purposes. To perform this survey, the top LTL carriers in United States are selected using the 2003 financial and operating statistics of auto carriers [6]. For the purpose of this survey, a generic email was prepared and sent to key contact persons or departments within each LTL company. The main goal of the research was discussed briefly. The journal paper [7] that was published based on this research was introduced as a reference to show that this is just a request for information that does not intend to advertise any product. The information regarding the technologies and the software that the company uses to manage and optimize the daily operations were requested. Fortunately, a number of responses were received from top players in LTL industry.

LTL carriers have been installing data communication units in their pickupand-delivery trucks to improve the efficiency and productivity of their operations. The volume and destination of shipments are estimated based on past trends, but typically are not confirmed until the pickup-and-delivery trucks return. However, there is considerable variability from day to day; for example, a shipper may call in an estimated shipment of 2000 pounds but the load's actual size may be 6000 pounds. In addition the decision time window for load planning is very short. Therefore having confirmed information on a shipment even one or two hours before the pickup-and-delivery truck returns to the terminal is enough to optimize the loading and dispatching of outbound trailers. Carriers are using data terminals (either in the truck or hand-held) to capture changes in shipment size and destination. These data can be sent immediately to the terminal, where they can be used to plan terminal operation and dispatching the outbound traffic. 
Some of the top LTL carriers (e.g. Con-Way Transportation Services, Inc.) do not acquire a commercial software package to manage their day to day operations. They have a software development center at their corporate IT department through which they develop their own dispatch, load allocation and routing management system to meet the very specific and unique needs of their operating environment. Other LTL carriers use commercial software to help them manage their fleet management process. Using online search and also based on the information received from LTL carriers a number of companies are selected that develop freight management solution to answer the needs of LTL industry. Here are two major features of the fleet management products that are provided by these companies:

1- Hardware: They develop a wireless fleet management system that provides real-time, two-way data communication between trucks and dispatching center. Additional features include vehicle tracking and vehicle position reporting using Global Positioning System (GPS). Using these state-of-the-art information networks, trucking companies move data as much as they move packages in order to reduce their overall fleet miles and improve their efficiency.

2- Software: They design applications that utilize the data provided by the information network to improve productivity and customer service. These products handle the operations as well as financial and administrative requirements of trucking companies to reduce their paperwork and chance of data entry errors. Using computerized mappings, dispatchers can access the detailed information on trucks and shipments locations in real time. Some of these commercial softwares are also being advertised as a tool to optimize the operational plan for LTL carrier. However, they do not provide any detail on their optimization process. Based on the limited survey that is conducted for the purpose of this research, it is not clear if there is any mathematical formulation that is being used to model the optimization problem. Most of these commercial applications seem to be powerful tools that help the dispatching centers to handle the job by providing more information and decreasing operator errors. 


\section{Motivation of Research}

Real-time decision making problems are playing an increasingly important role in the economy due to advances in communication and information technologies that now allow real-time information to be quickly obtained and processed. The intelligent transportation system (ITS) technologies increase the flow of available data and offer opportunities to control the transportation in real-time. Some research efforts have considered the real-time acceptance/rejection of the requests for service, dispatching, assignments, routing and rerouting of the vehicle (Regan, Mahmassani, and Jaillet [8,9,10], Yang, Jaillet and Mahmassani [11,12], Jung [13], Mahmassani, Kim, and Jaillet [14], Gendreau et al. [15,16,17], and Kim, Mahmassani and Jaillet $[18,19])$. All of these studies focused on truckload trucking operations and very little work has been done to take advantage of the real-time information in decision making for LTL carriers in a dynamically changing environment.

LTL motor carriers generally accept as many loads as they can, and rely on the human dispatchers to make the appropriate decisions on the basis of experience and the available real-time information. If they use tactical planning strategies and create the load plan, their operation would be under go-when-filled strategy subject to a minimum frequency constraint (typically 3-5 trailers/week) to manage the level of service. For the LTL carrier the main objective is to minimize the operating cost, so they try not to dispatch half full trucks. That is why a typical shipment spends more than $50 \%$ of its total travel time at terminals for loading which causes customer dissatisfaction.

Auto carriers are one class of motor carriers that work on hub-and-spoke networks. They don't guarantee pickup and delivery dates (after booking the request, they provide an estimate transit time that the shipment will take). Customers can check the status of the progress of shipment and track them either online or by calling the carrier. There are a large number of online complaints that have been posted by dissatisfied customers, mostly because of delays in delivery. Badbusinessbureau.com is one of many websites that allow individuals to post their opinions and comments against companies and businesses that have treated them unfairly. On this website, there are hundreds of complaints about auto carriers that transport vehicles 
throughout the United Sates and operate on hub-and-spoke networks. The followings are some of the recent complaints that have been posted online, and show how the companies failed in making real-time decisions for their operations [20]:

Posted on: $10 / 24 / 2005$

Company: AAAdvantage Auto Transport (Arizona)

"My uncle purchased a car for me and the only thing left for me to do was get it delivered from Pittsburgh, PA to California. After checking out a number of auto transporting companies, and searching for the one, which charged the least amount, I discovered AAAdvantage auto transport. I was told that the car would be picked up in Pittsburgh within 3 days and delivered within 14-16 days. I gave this company \$1,046 on Sept 21. On Oct. 21, I found out that my car had never been picked up and it was still at the dealership. I spoke with another customer service rep that notified me that my car should be arriving by Nov.15..."

Posted on $8 / 13 / 2005$

Company: Major Transport (New York)

"My car was scheduled for pick up in New York to be delivered to Live Oak, TX. I called on $8 / 1$ and was told $8 / 3$ would be the pick up date. They stated that the estimated delivery date would be in about 5-7 business days. On 8/9 the dispatcher called me back to state the driver was in Virginia picking up another car and I would be the next stop made. On 8/12 after my vehicle was still not delivered I contacted them once again and was told that it will arrive by $8 / 25$ or within a total of 21 business days. Nowhere in the contract that I did not sign, states that it could take up to 21 business days for my vehicle to reach the final destination point..."

Posted on: 7/10/2005

Company: A-1 Auto Transport (Nevada)

"On March 4, 2003, I contracted A1 Auto Transport of Carson City, Nevada to transport a vehicle from Indianapolis, IN to Pacific, WA. I received quotes ranging from $\$ 850$ to $\$ 1100$. Al offered to ship the vehicle for $\$ 1090$. They assured me they could pick up and ship my vehicle within 3-5 days, and put a latest pickup date of March 10, 2003 on the contract. On March 10th I had not heard a word from Al, so I contacted the company. I received an e-mail back saying the car would not be picked up until Friday, March 14th? This pushed their 3-5 day window to 10 days. I could've contracted through any other company for a less rate and been assured 10 days..." 


\section{$\underline{\text { Contributions }}$}

The majority of studies related to LTL networks are related to load planning, and service network design. Using tactical planning only that part of demand can be satisfied which has a high degree of certainty (which is $60-80 \%$ of total demand). The rest of the demand is revealed dynamically. Decision makers (dispatchers) must be able to answer these questions in real-time:

- How to accept/reject a request?

- How to route a shipment through the LTL network?

- How to assign the shipments to trucks?

- How to route the trucks?

- How to schedule the trucks (what is the best policy for trailer closing? What is the best choice of fill-rate requirement?)

- How to assign drivers to trucks?

These issues can be addressed using an operational planning practice, which is the focus of this study. The main contributions of this research are:

- To develop a mixed integer programming (MIP) model to optimize the combined dynamic shipments routing and dynamic trucks routing and scheduling for LTL trucking operations

- To introduce a heuristic algorithm to solve the MIP problem

- To find a lower bound for the MIP problem, and check the quality of the solution provided by the heuristic algorithm

- To propose a decision making procedure to handle the requests for LTL shipments in an environment that changes dynamically

- To develop a set of simulation experiments to evaluate the effect of decision making techniques on LTL operations, and compare them to what the carriers do in practice (as the benchmark) 


\section{Organization of the Dissertation}

Chapter 2 begins with a brief presentation of different levels of planning for LTL trucking. It is followed by the review of literature that is related to tactical and operational planning. In each part previous studies have been grouped based on the problems they try to solve. For each group of studies, a review is presented in chronological order.

Chapter 3 presents a mathematical formulation for the proposed model. It starts with a short introduction that highlights the difference between rail operations and LTL trucking operations. The mathematical model assumptions, notations, decision variables, objective function, and constraints are presented as well as 3 extensions to the original formulation to capture the non-homogeneous fleet, the driver routing and the additional waiting costs.

In Chapter 4 a procedure is proposed to make acceptance/rejection decisions for LTL motor carriers. Fast-acceptance techniques are proposed that can be used under high demand condition to increase the ability of handling the requests when solving the optimization problem is computationally expensive. The discussion is followed by presentation of a decision support application that is developed based on the proposed decision making procedure.

Chapter 5 presents the sensitivity analyses results based on 2 sets of numerical experiments. The main goal of performing the first set of numerical experiments is to check the accuracy of the mathematical formulation and 3 computer programs that are discussed in Chapter 4. The second set of numerical experiments is conducted on a 10-terminal network to study the system behavior and sensitivity of the solution with respect to changes in the contributing factors.

One major step in all decision making procedures is to solve the shipment and truck routing problem repeatedly. In Chapter 6 three heuristic solution algorithms are proposed to solve the MIP problem. The first 2 approaches are based on a search algorithm, which tries to find the best paths to route the shipments. The last approach uses a graphic partitioning to reduce the size of the problem which is solved using CPLEX. In order to evaluate the effectiveness of the solution algorithms, three benchmark solutions are proposed; "do-nothing", "literature-reported operation" and 
the "lower bound". The performance of the proposed solution methods are analyzed in the next Chapter.

Chapter 7 reports the results of numerical experiments on medium and largesize networks. The first set of numerical experiments is conducted on a 10-terminal network to check the quality of the solution provided by the proposed algorithm to solve the MIP problem. In the second numerical experiment the performance of the proposed methods are analyzed using a more realistic large-size 17-terminal network. Besides, a simulation framework is introduced and the effectiveness of the proposed decision making procedure is evaluated.

Chapter 8 summarizes the research objectives, contributions and achievements. Other areas for research on LTL trucking operation are addressed and a list of recommended future research is provided in this Chapter. 


\section{Chapter 2: Literature Review}

This Chapter discusses the major research efforts and studies that have been conducted on truckload trucking, less-than-truckload-trucking, tactical and operational planning for LTL trucking operations and finally the multi-commodity network flow problem and solution algorithms.

\section{$\underline{\text { TL Trucking }}$}

As discussed earlier, trucking services are classified as truckload (TL) and less-than-truckload (LTL) based on the type of operations. In truckload trucking one truck is assigned to each customer. When a customer requests for pickup and delivery of a load, the carrier makes the decision to accept or reject the request. If the load is accepted, a truck moves empty to pick it up and moves the load to its destination. After unloading, the truck is ready for a new assignment. The truck can be assigned to a new load, or can be moved empty to a new location, or can be kept idle at the same location. During the past decade many studies focused on real-time dispatching, assignment, routing and re-routing of vehicles for TL trucking operations. Due to similarities between TL and LTL trucking operations the literatures on TL trucking are also discussed in this section. The ideas that have been introduced for TL trucking operations can be helpful in development of decision support tools for other transportation systems, including LTL trucking.

Regan et al. [8] identified the potential uses of real-time information for the efficient management of truckload carrier operation. They proposed and analyzed the en-route diversion strategies in response to unknown customer demand. They used single-vehicle simulation experiments to compare diversion strategies versus two base cases (assignment in the exact order of arrival, and assignment after resequencing the demands waiting for service). Their findings suggested that 
meaningful potential exists for reducing travel distances and improving efficiency by using diversion strategies.

In 2 other studies Regan et al. [9,10] developed a simulation framework to evaluate the performance of alternative load acceptance and assignment strategies using real-time information. They considered different types of geographic region, demand arrival patterns and number of vehicles as the elements of the simulation framework. A number of load acceptance and assignment strategies were analyzed. The results for single-vehicle simulation showed that less restrictive acceptance rules are more effective than restrictive ones. The diversion strategies performed well especially when time windows for acceptance are short. The results also showed that under more realistic demand and larger fleet size the flexible assignment strategies work well, which means that company can provide much better service to customers and at the same time remain profitable. So they suggested that a hybrid system which would choose the assignment strategy based on current congestion level of system may result in increased efficiency.

Yang et al. [11] studied the on-line algorithm for truck fleet assignment and scheduling under real-time information. They introduced a mathematical formulation of truckload pick-up and delivery problem with time windows. The problem was formulated as an off-line assignment problem. An on-line strategy (which was called "optimal") solves the off-line problem in a rolling horizon framework. The problem is solved using CPLEX solver each time a new request is received. A set of simulation experiments were conducted to compare the heuristic strategies and the optimal strategy. The results showed that the strategies with re-sequencing loads outperform strategies without re-sequencing. Optimal strategy outperformed all the others, but required the most time to execute.

Mahmassani et al. [14] presented a hybrid strategy to solve dynamic commercial fleet management problem. In the first stage the incoming load is assigned to a vehicle based on some heuristic rules. The computation is fast, so the operator can respond the customer. In the second stage, after initial assignment, the operator has enough time to reconsider the vehicles' routes and schedules before a new load request is received, so the reassignment is done using optimization methods. 
In this study, some strategies were suggested to control the size of the problem, such as merging the close demands. Simulation experiments were performed to test these strategies. The results showed that the merging of close demands reduces the computing time in large problems.

Kim et al. [18] studied the dynamic truckload truck routing and scheduling in oversaturated demand situations (when the demand exceeds the system's average capacity). Three assignment techniques were developed and applied to the problem (all use a mixed integer programming model). To maximize the computation capability within a given time, a dynamic adaptive dispatching strategy was proposed to arrange the application of three types of assignments. A simulation framework was developed to evaluate the performance of the algorithm. They showed that the dynamic adaptive dispatching strategy significantly improves profit and reduces response time. The results suggested that, in oversaturated demand environment, keeping the number of waiting jobs in the queue below the holding capacity is more beneficial than accepting and holding as many demand as possible.

Yang et al. [12] focused on Real-time multi-vehicle truckload pick-up and delivery problems. They used a mixed integer programming formulation for the offline version of the problem. They proposed a new strategy which assumes some knowledge about the probability law of future job pickup (and delivery) location. They compared this new strategy and 4 other rolling horizon strategies (previous studies) under varying traffic intensities, degrees of advance information and varying degrees of flexibility for job rejection decision. The new re-optimization policy is shown to systematically outperform the other heuristic strategies that work based on simple local rules.

Jung [13] used a genetic algorithm approach to solve the vehicle routing problem with time-dependent travel times. The author considered multiple vehicles with different capacities, real-time service requests, and real-time variations in travel time. The problem was formulated as a mixed integer programming problem. A heuristic method (GA) was proposed to solve the MIP problem along with a methodology to find a lower bound. Optimal solutions, lower bound solutions, and genetic algorithm solutions were compared on randomly generated test problem. The 
author also developed a time dependent shortest path algorithm and used a simulation framework to compare the deterministic, and time-dependent routing plan.

In one of the latest studies on dynamic truckload routing, scheduling and load acceptance, Kim et al. [19] considered high priority demands. The delivery service is classified into two types (Priority, and Regular service). A previously presented mixed integer programming model is developed for two-class demand situation. They proposed a dynamic acceptance/rejection policy. Simulation experiments were conducted to evaluate the performance of proposed strategy. The results showed that the new policy significantly improves the total profit and reduces the delay compared to benchmark policies. They suggested a future research direction would be to consider multiple pickups/deliveries for less-than-truckload delivery service considering the truck capacity constraints.

This section discussed some of the most significant research efforts and different approaches to control the truckload trucking operations using real-time information. In these studies the real-time acceptance/rejection of the requests, dispatching, assignments, routing and rerouting of the vehicle were discussed. Despite the differences between the truckload and less-than-truck operations, they have some similar components. Both use a group of drivers, a fleet of trucks, a set of terminals and a dispatching center to respond to the demand which changes in a dynamic fashion. Therefore, the ideas that have been introduced for TL trucking operations can be helpful in development of decision support tools for LTL trucking.

\section{$\underline{\text { LTL Trucking }}$}

During the past two decades, several approaches have been introduced to tackle the management problems for less-than-truckload motor carriers. Delmore et al. [21], Golden and Assad [22], Crainic and Laporte [23,24], and Roy [25] reviewed the optimization-based operations research methodologies that have been proposed in the literature and identified the major trends, challenges and developments. From a planning point of view one can classify this research into 3 levels: strategic, tactical 
and operational. The goals, rules, policies and guidelines are defined at higher levels and move from strategic planning toward operational planning. Data and information that is required for decision-making is moving backward from the lowest level (operational level) to strategic level.

- Strategic (log-term) Planning

This is the highest level of management and typically concerns the design of transportation system. It usually needs a large investment over a long-term horizon, and must be revised periodically to capture the changes in the environment. Strategic plans aim to:

- Identify the fundamental elements of a transportation system, such as demand, and supply

- Design the physical network (i.e. line operations network), and design the location of major facilities (i.e. end-of-line, and hubs)

- Tactical (medium-term) Planning

The main goal at this level is to determine the required medium-term activities to achieve the best possible performance of the system. The tactical plans aim to:

- Adjust the system capacity based on demand forecast

- Design the service network

- Determine the routes, service schedules, and vehicle/shipment routing

- Operational (short-term) Planning

This level of planning concerns very short-term day-to-day operations. It is performed by local management, and dispatchers in a highly dynamic environment. Based on the transportation plans developed at the tactical level carriers assign drivers and vehicles and update transportation schedules in response to daily variations in demand, and in resource availability. The operational plans aim to:

- Dynamically decide to accept or reject the requests for service 
- Dynamically handle the demand, and allocate the resources (i.e. route the shipments, assign the vehicles to the loads, and assign the drivers to the vehicles)

- Dynamically adjust the schedules for maintenance activities

\section{$\underline{\text { Tactical Planning }}$}

Literatures related to the LTL trucking operations at the tactical level can be divided into 2 separate groups. Some researchers focused on solving the load planning problem while others analyzed the routing of freight shipment through many-to-many logistic networks. This part summarizes both groups of studies.

The load planning model for LTL motor carriers introduced by Powell and Sheffi [26,27], and Powell [28] uses a frequency service network design formulation where level of service constraints are represented through a set of minimum frequencies on links. In these studies the load-planning problem is formulated as a large-scale mathematical model. They decompose the problem hierarchically into a network design problem, and a series of subproblems determining the routing of LTL shipments, the routing of TL shipments and the routing of empty trailers to balance the network. They solve the network design problem as a structured local improvement heuristic that successively adds and drops links to and from the network in search of opportunities to reduce costs. The model and the solution method are at the core of an interactive decision support system dubbed APOLLO, and have been implemented at a major US less-than-truckload trucking company. Impressive results are reported with respect to the impact of the system both on load planning operations and strategic studies of potential terminal location.

In a research by Powell and Koskosidis [29] the service network is taken as fixed, and they focus instead on the shipment routing subproblem to minimize the total transportation and handling costs, subject to two key constraints: Minimum frequency for service between two terminals and the tree constraint. The latter is derived from real world consideration, which requires that instructions to the field be 
of the form "freight at terminal $\mathrm{X}$ headed for destination $\mathrm{Y}$ must move next to terminal Z". A solution approach is developed using a shortest path based formulation and a local improvement heuristic is presented to solve the problem. They also developed a separate set of primal-dual algorithms which provide both upper and lower bounds. The effectiveness of the algorithm has been evaluated through a set of numerical experiments.

Crainic and Roy [30] studied the design of driver routes for the LTL motor carrier industry. Forecasts must be made for that part of the demand for transportation services which is considered to be stable over a given planning horizon. These market forecasts are then used as inputs to the tactical planning process that produces a load plan for the stable part of demand for LTL services. Such a plan can be established, for example, by using the interactive optimization system, APOLLO, based on ideas proposed by Powell and Sheffi [28], or the NETPLAN software developed from a tactical planning model proposed by Roy and Delmore [31] and based on a methodology by Crainic and Rousseau [32]. The next step is to design the regular driver routes. The results indicate that the proposed methodology can provide assistance to motor carriers in making better use of their resources and improve their productivity.

Daganzo [33] examined the structure of many-to-many logistics networks. Using as little data as possible this study attempts to answer macroscopic questions such as: how many terminals should be used? Should they be used at all? What should be the frequency of service? They show that near-optimal network structures can be characterized by two dimensionless constants, which can be determined from the data. They also highlight the difference between many-to-many and one-to-many (or many-to-one) networks, the role breakbulk terminals play in many-to-many logistic networks and how using transshipments can reduce cost.

A study by Hall [34] used two measures of consolidation (number of terminals and number of links) to compare 4 strategies for routing shipments through hub-andspoke network. They showed that decreasing the number of links and the number of terminals leads to increased consolidation and reduced operating and shipping costs. They discussed the best application for each strategy. One strategy is two-terminal 
routing, which means each shipment passes through two terminals. This strategy is attractive when the number of origins and the number of destinations is large, and it is a logical strategy for LTL carriers.

In the routing schemes developed by Daganzo [33], and Hall [34] the shipments are consolidated either at the origin of the freight or at the origin terminal. The methodology proposed by Akyilmaz [35] allows the consolidation of the shipments at intermediate breakbulk terminals and it explicitly specifies the routing of the shipments. Solution results of the numerical experiments show that the algorithm offers an efficient and reasonably accurate method for the routing of LTL shipments via the intermediate terminals.

\section{Operational Planning}

Manufacturing, service and transportation companies try not only to cut their logistics costs, but also to compete on service differentiation. Not surprisingly, a fast growing body of research focused on time constrained routing and scheduling (Desrosiers et al. [36]). The time dimension has been considered in these problems in the form of customer-imposed time window constraints. This part discusses the literature related to planning at the operational level, for which the time dimension is a major element.

Multi-commodity network flow problems (MCNF) can be used to model many real-world problems, including the freight distribution problem of LTL motor carriers. MCNF problem with linear cost is simply a linear program but when it is used to model real-world problems the mathematical programming problem is so large that cannot be solved using the revised simplex method. The MCNF problem and its solution algorithms have been extensively studied in the past. Assad [37] and Kennington [38] provide reviews on these solution algorithms. A summery on the MCNF problem and solution algorithms are provided in the last section of this Chapter. 
Haghani [39] used MCNF to model the combined train routing and makeup, and empty car distribution problem. The proposed formulation results in a large-scale mixed-integer programming problem with non-linear objective function and linear constraints. A heuristic decomposition technique is developed to solve the model. This solution procedure decomposes the problem to smaller sub-problems based on the type of decision variables.

Barnhart and Sheffi [40] presented a primal-dual heuristic solution approach for MCNF problems. To demonstrate the effectiveness of the solution strategy, a large-scale freight assignment problem in LTL trucking industry is formulated as a MCNF problem. Two linear programming based exact solution strategies are unable to achieve even an initial solution for the problem (because of excessive memory requirements). The proposed heuristic, however, determines a solution. They used a smaller test problem to compare the performance of the proposed heuristic with that of the exact procedures.

Similar to the previous work, Farvolden et al. [41] present a new solution approach for the MCNF based on both primal partitioning and a decomposition technique which simplifies the computations required by the simplex method. This solution was developed specifically for problems with the characteristics of the LTL shipment routing problem. This work was followed by the study done by Farvolden and Powell [42], which provides a combined formulation of the service network design problem and shipment routing problem in a dynamic setting. They developed local-improvement heuristics to solve the resulting MCNF problem. The heuristics are based on subgradients derived from the optimal dual variables of the shipment routing subproblems. The empty balancing is considered as an independent subproblem and is not addressed in this study.

Kleywegt and Papastavrou [43] studied the acceptance and dispatching policies for LTL carriers. This paper formulates a dynamic and stochastic distribution problem (DSDP) that combines acceptance and dispatching policies for LTL distribution operations over a network. They developed a Markov decision process model and proposed an algorithm that utilizes the structure of the problem. One important assumption was that after loads have been consolidated at their origin 
terminals they are shipped directly to their destinations. Therefore their model does not consider intermediate cross-docking besides that at origin and destination terminals.

The LTL carriers operate on a hub-and-spoke freight transportation network and simulation is a powerful too that can be used to study the impact of dynamic decision making on these type of systems. In a work done by Cheung and Muralidharan [44], a comprehensive simulation model is developed to capture the relationship between the LTL network configuration, load planning, work rules and trailer closing policies. Their simulation results suggest that changing the shipment routes and the trailer closing rules in a dynamic fashion has a large impact on the level of service. Based on the results they also formulate the trailer closing policy for one OD pair as a dynamic programming model.

Cheung and Muralidharan [45] considered a shipment routing strategy for the priority shipments on an LTL network. By using a network formulation, this strategy can be approximated by finding a dynamic shortest path over a stochastic network. They show that the expected travel times from all nodes to a destination node can be computed very quickly via a dynamic programming algorithm off-line. These expected travel times capture the ability to change shipment routes when some of the uncertainty is revealed over time. Their numerical experiments indicate that this adaptive routing strategy allows the priority shipments to reach their destination faster for the shipments that have long loading times and that are most likely to miss their due date.

\section{MCNF problem and solution algorithms}

The multi-commodity network flow (MCNF) problem is defined for a network where more than one commodity needs to be transported. Unlike SCNF problem, in multi-commodity flow problems no commodity can be transformed into another commodity, so each has its own flow conservation constraints. All 
commodities use the same network and resources that are represented by arc capacities. There are three different types of MCNF problems [46]:

- Max MCNF problem, which maximizes the sum of flows for all commodities between their origin and destinations.

- Max-concurrent flow problem, which maximizes the fraction of satisfied demands for all commodities.

- Min-cost MCNF problem, which finds the flow which satisfies the demands of all commodities with minimum cost (considering the capacity constraint on all arcs). In this research the minimum cost MCNF problem has been used.

Due to the existence of bundle constraints that limit the total flow of all commodities to arc capacities, the MCNF problem is much more difficult than the SCNF problem. Solving the integer MCNF problem is NP-complete [47, 48].

In the last four decades the MCNF problem was the main motivation for many operations research studies. For example, the Dantzig-Wolfe decomposition algorithm [49] was originated from the study done by Ford and Fulkerson [50] on Maximal multi-commodity network flow problem. The MCNF mathematical model was involved in many real-world OR applications. The most recent applications of MCNF models are in telecommunication network routing [51-54], routing and scheduling in transportation and logistics [55-59], production scheduling and planning [60, 61], VLSI design [62-65], traffic equilibrium [66, 67], graph partitioning [68-72], and network design [73-77]. Wang [46] provides reviews on some of these applications.

The MCNF problem has been presented using two different formulations. Both formulations will be referenced in this dissertation. In this section simple notations are used to present both forms along with the discussion of their major differences.

Given a network, which consists of a set of nodes $(N)$ and a set of $\operatorname{arcs}(A)$, a set of commodities $(k \in K)$ must be shipped from their origin to their destinations. It is assumed that there is a cost $C L(k, i j)$ associated with shipping commodity k on arc 
$i j \in A$. Capacity of arc $i j$ is assumed to be equal to $U(i j)$ that limits the total flow on arc $i j \in A$. Associated with each commodity $k \in K$ at each origin/destination node $i \in N$, there is a parameter $B(k, i)$ that represents the demand/supply of commodity $k$ at that node. The decision variable is $X L(k, i j)$, which is the flow of commodity $k \in K$ on arc $i j \in A$. Given the above notations and definitions the "Node-Arc" (link-based) formulation of MCNF is as follows:

Minimize

$\sum_{k \in K} \sum_{i j \in A}[X L(k, i j) . C L(k, i j)]$

Subject to:

$$
\begin{array}{lr}
\sum_{i j \in A} X L(k, i j)-\sum_{j i \in A} X L(k, j i)=B(k, i) & \forall i j \in A, \forall k \in K \\
\sum_{k \in K} X L(k, i j) \leq U(i j) & \forall i j \in A \\
X L(k, i j) \geq 0 & \forall k \in K, \forall i j \in A \\
X L(k, i j)=0,1,2, \ldots & \forall k \in K, \forall i j \in A
\end{array}
$$

Parameter $B(k, i)$ is positive when $i$ is an origin node. It is negative when $i$ is a destination node, and it is equal to zero when it is neither origin nor destination node. The objective is to minimize the total cost (1), while the flow conservation constraints (2) and bundle constraints (3) are satisfied. Constraints (4) and (5) represent the nonnegativity and integrality.

Tomlin [78] proposed an alternative form of the min-cost MCNF problem. This form, which is called "Arc-Path" (Path-based) formulation, can be obtained by extending the flow decomposition theorem [79] to multi-commodity flows as follows [80]:

“Flow Decomposition Theorem:

Any non-negative feasible flow can be decomposed to the sum of cycle flows and path flows. Path flows originates from one supply node and destines to a demand node. The decomposition is not necessarily unique. 
By assuming that all unit cost are non-negative, there will be no cycles with negative cost, and the optimal solution meets the following proposition:

Proposition: An instance of the integer multicommodity problem in which all cycles have non-negative cost and the set of feasible solutions is non-empty has an optimal solution such that all cycle flow are equal to zero. The same can be stated about the optimal solution of the linear relaxation of the problem.

Proof: In any solution, the cycle flow can take any positive value without violating the flow conservation constraints. On the other hand, as bundle constraints are of type " $\leq$ ", any positive cycle flow can be driven to zero in a way such that all constraints are still satisfied and the objective function decreases monotonically, because all cycle variables have a non-negative coefficient in the objective function."

$P(k)$ is assumed to be the set of all paths between the OD pairs of commodity $k$. Cost $C P(k, p)$ is associated with shipping commodity $k$ on path $p \in P(k)$. If arc $i j$ belongs to path $p$ of commodity $k$, then $D(p, k, i j)$ is equal to 1 (and zero, otherwise). The decision variable is $X P(k, p)$, which is the flow of commodity $k \in K$ on path $p \in P(k)$. Given the above notations and definitions the Arc-Path formulation of $\mathrm{MCNF}$ is as follows:

$\sum_{k \in K} \sum_{p \in P(k)}^{\text {Minimize }}[X P(k, p) \cdot C P(k, p)]$

Subject to:

$$
\begin{array}{lr}
\sum_{p \in P(k)} X P(k, p)=1 & \forall k \in K \text { (7) } \\
\sum_{k \in K} \sum_{p \in P(k)} D(p, k, i j) . X P(k, p) \leq U(i j) & \forall i j \in A \text { (8) } \\
X P(k, p) \geq 0 & \forall k \in K, \forall p \in P(k) \text { (9) } \\
X P(k, p)=0,1,2, \ldots & \forall k \in K, \forall p \in P(k) \text { (10) }
\end{array}
$$

The objective is to minimize the total cost (6), while the flow conservation constraints (7) and bundle constraints (8) are satisfied. Constraints (9) and (10) represent the non-negativity and integrality. 
The node-arc formulation has $|K||A|$ variables and $|N||K|+|A|$ constraints. In the worst case, $|K|$ is $O\left(|N|^{2}\right)$ and the node-arc form will have $O\left(|N|^{3}\right)$ constraints. This makes the memory management inefficient and the computations would be more difficult compared to arc-path formulation. On the other hand, the arc-path formulation does not have more than $O\left(|N|^{2}\right)$ constraints, but the number of variables grows exponentially with the size of the network. Usually column generation techniques are used to tackle such problems with large number of decision variables.

The MCNF problem has a block-angular structure, for which there are many solution methods that have been suggested in the literature. In this study, due to the characteristics of the commodities and the special connections between them, the problem cannot be treated as a general MCNF problem. On the other hand an efficient problem-specific solution algorithm is needed to be implemented for practical use in dynamically changed environment.

Basis partitioning methods are solution algorithms based on which the simplex basis matrix is partitioned and the network is exploited in order to make the inversion of the basis more efficient. In resource-directive methods, a capacity is assigned to each arc for each commodity and the original problem becomes equivalent to a resource allocation problem, for which there are a number of solution algorithm in the literature.

Lagrange relaxation and Dantzig-Wolfe decomposition are among the pricedirective methods where by associating the bundle constraints with a penalty function the MCNF problem would be decomposed into a series of easy SCNF problems.

Primal-dual method starts with a feasible dual solution and then uses the complementary slackness to build the primal feasibility subproblem, iteratively. Using the optimal dual solution of the subproblem it improves the current dual solution and continues until there is no primal infeasibility.

Interior point methods are suitable algorithms to tackle very large linear programs such as MCNF problems. This algorithm, which has been inspired by Karmarkar's algorithm [81], is a fast algorithm that searches in the interior of the feasible set rather than on the boundary (like the simplex methods). 
There are a number of comprehensive survey papers that have been published during the past 3 decades and focused on solution techniques and computational results for MCNF problems (Assad [37] and Kennington [38]). Previous computational experiments suggested that the price-directive methods are better techniques, in general, compared to the resource-directive and basis-partitioning methods. During the past decade, there has been also a significant improvement in the quality of solution provided by solver packages (i.e. CPLEX) by implementing some of the solution techniques (i.e. interior point methods) and impressive results have been reported in the literature.

This Chapter discussed some of the most significant research efforts focused on truckload trucking, less-than-truckload-trucking, tactical and operational planning for LTL trucking operations and finally the multi-commodity network flow problem and solution algorithms. The intelligent transportation system (ITS) technologies increase the availability of data and offer opportunities to control the transportation operations in real-time. As discussed earlier in this Chapter some efforts have considered real-time acceptance/rejection of shipping requests, but mostly focused on truckload trucking operations. This study tries to use real-time information in decision making for LTL carriers in a dynamically changing environment. In the next Chapter, based on the general structure of MCNF problems, a mathematical model is developed to capture the dynamic shipment and vehicle scheduling problem in LTL trucking operations. 


\section{Chapter 3: Mathematical Model}

The problem is formulated as a capacitated Multi Commodity Network Flow problem (MCNF). The capacity of a link is the product of the number of loaded trucks on each link and the capacity of each truck.

The operation of commercial airlines and trains are similar to that of LTL trucking. These are different transportation modes, but they all operate on hub-andspoke networks and take advantage of shipment/customer consolidation. One major difference between trucking operation and airline or train operation is that trucks have more flexibility in terms of the routing path/link and schedules.

As discussed in the previous section, Haghani [39] developed a MCNF model for the rail operations which is a type of consolidation transportation. That study has been taken as the main reference to develop the mathematical model for the LTL trucking operations. Despite the similarities between the rail operations and the LTL trucking operations, there are some major differences between the two. The main components of those systems and the relationship between them are different. Decision making for rail operations includes assigning the blocks of freight cars to trains, routing trains, and redistribution of empty freight cars but the decision making problem for the LTL trucking operations consists of routing the shipments, assigning the shipments to trucks, routing the loaded trucks, and redistribution of the empty trucks.

This chapter presents the mathematical formulation for the dynamic shipment and vehicle routing and scheduling problem for LTL trucking operation. The discussion follows by presenting 3 extensions to the original formulation to capture the non-homogeneous fleet, the driver routing and the additional waiting costs.

\section{Assumptions}

At the arrival time of a request the company is given the pick-up location, the delivery location, the earliest pick-up time, and the latest delivery time for each 
shipment. The company can either accept or reject a service request within a small amount of time. The revenue generated from the delivery of each load is proportional to the distance between its pick-up and delivery locations. There will be a penalty for completion beyond the latest delivery time. The penalty is proportional to the delivery distance and amount of delay. For serving the sequence of requests, there are some additional operating costs proportional to the loaded/empty distance traveled by trucks in order to serve the accepted request. Note that in this study, both terms "full truck" and "loaded truck" refer to a "non-empty truck". Here is the list of assumptions:

- Number and location of end-of-line and break-bulk terminals are known

- Service network is known (Tactical planning is already done)

- End-of-line terminals are taken as the points of origination and termination (The local pickup/delivery problem is not solved in this study)

- Travel times are fixed (since LTL companies operate on the intercity network)

- There is a time window for each demand within which the demand should be served, otherwise there will be a penalty (soft time windows)

- Demand info (origin, destination, time windows) are revealed dynamically

- Number of vehicles (fleet size) is known

\section{Time-Space Network}

In this research a time-space network is used to represent the model. Timespace network is an effective modeling framework for scheduling and routing problems. These models, which are also known as dynamic network models, were developed in the 1950's. Due to the logical setup of the time-space networks one can clearly describe the structure of the problem using these models.

In this research, the space is the region for which the carrier provides the LTL service and is represented in one dimension and time lies in the other dimension. 
Each node within the network shows an event taking place in a specific terminal at a specific time. Each arc represents the linkage among different nodes. The next section presents the notations as well as the definitions of different elements in both physical as well as time-space network.

\section{$\underline{\text { Notations }}$}

- Physical Network

The physical network consists of a set of nodes (terminals) and a set of links as shown in Figure 5.

$$
\begin{array}{ll}
N & =\text { Set of all nodes (end-of-line, and breakbulk terminals) } n \in N \\
L & =\text { Set of all links } l \in L \\
T T(l) & =\text { Travel Time on link } l \in L
\end{array}
$$

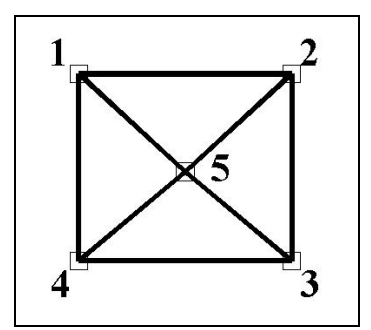

Figure 5 - Physical network

\section{- Time-Space Network}

The time-space network consists of a set of nodes (terminals) for each time period, a set of routing links for all connected terminals for each time period, and finally a set of waiting links for each terminal between each two consecutive time periods as shown in Figure 6.

$$
\begin{array}{ll}
t & =\text { Time Period } \\
T & =\text { End of time horizon } \\
L R(t) & =\text { Set of all routing links that end at time period } t \in[0, T] \\
L W(t) & =\text { Set of all waiting links that end at time period } t \in[0, T]
\end{array}
$$


$L E(n, t)=$ Set of all links that end at node $n \in N$, at time period $t \in[0, T]$

$L B(n, t)=$ Set of all links that begin at node $n \in N$, at time period $t \in[0, T]$

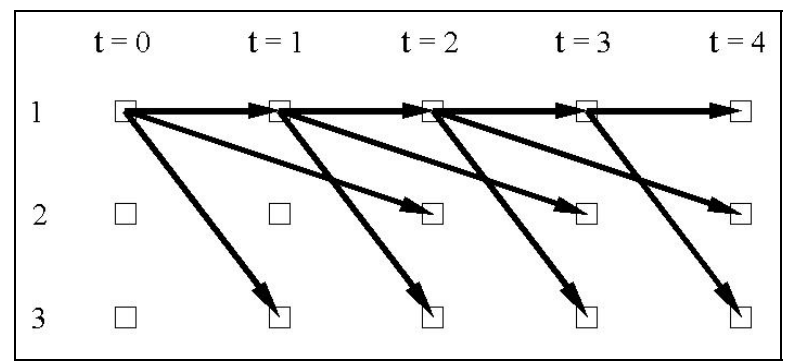

Figure 6 - Time-space network (3-terminal network)

- Shipments

$$
\begin{array}{ll}
S & =\text { Set of all shipments } \\
W(s) & =\text { Weight (size) of shipment } s \in S \\
O(s) & =\text { Origin of shipment } s \in S \\
D(s) & =\text { Destination of shipment } s \in S \\
T E(s) & =\text { Earliest pickup time for shipment } s \in S \\
T L(s) & =\text { Latest delivery time for shipment } s \in S
\end{array}
$$

- Trucks

$W F \quad=$ Capacity of a truck

$W E \quad=$ Minimum fill rate of a truck

$S E(n, t)=$ Supply of empty trucks on node $n \in N$ at time period $t \in[0, T]$

- Cost Functions

$C F(l) \quad=$ Full (loaded) truck routing cost over link $l \in L R(t)$

$C E(l) \quad=$ Empty truck routing cost over link $l \in L R(t)$

$\mathrm{CH}(s) \quad=$ Handling cost for shipment $s \in S$

$C W(s) \quad=$ Waiting cost for shipment $s \in S$ 
$C N(s, n)=$ Penalty cost for delivery failure by the end of the time horizon (for shipment $s \in S$ ends up being at node $n \neq D(s)$, at $t=T$ )

$C L(s) \quad=$ Penalty cost for late delivery for shipment $s \in S$

- Decision Variables

$X S(s, l)=$ Flow of shipment $s \in S$ on link $l \in L R(t)$

$X F(l) \quad=$ Flow of full (loaded) trucks on link $l \in L R(t)$

$X E(l) \quad=$ Flow of empty trucks on link $l \in L R(t)$

\section{Objective Function}

- Routing Costs

Routing costs are equal to the total flow of full (loaded) and empty trucks over a routing link multiplied by the associated cost over link $l(C F(l)$, and $C E(l))$ summed over all routing links in all periods of the time-space network.

$\sum_{t=0}^{T}\left[\sum_{l \in L R(t)}[X F(l) \cdot C F(l)]+\sum_{l \in L R(t)}[X E(l) \cdot C E(l)]\right]$

\section{- Handling Costs}

Handling costs are equal to the total flow of shipments $s$ over a routing link multiplied by the associated cost over link $l(C H(s))$, summed over all routing links in all periods of the time-space network.

$\sum_{t=0}^{T} \sum_{l \in L R(t)} \sum_{s \in S}[X S(s, l) . C H(s)]$ 


\section{- Waiting Costs}

Waiting costs are equal to the total flow of shipments $s$ over a waiting link multiplied by the associated cost over link $l(C W(s))$, summed over all waiting links in all periods of the time-space network.

$\sum_{t=0}^{T} \sum_{l \in L W} \sum_{s \in S}[X S(s, l) . C W(s)]$

\section{- No-Delivery Penalty Costs}

No-delivery penalty costs are equal to the total flow of shipments $s$ over all links that end at node $n$ at the end of the time horizon, multiplied by the associated cost ( $C N(s$ , n) ), summed over all nodes other than the destination for $s(N-\{D(s)\})$, for all shipments.

$$
\sum_{s \in S} \sum_{n \in N-\{D(s)\}}\left[\left(\sum_{l \in L E(n, T)} X S(s, l)\right) \cdot C N(s, n)\right]
$$

\section{- Late-Delivery Penalty Costs}

Late-delivery penalty costs are equal to the total flow of shipments $s$ over all links that end at this destination nodes $(D(s))$ at time period $t$, multiplied by the difference between the actual delivery time and the latest delivery time $(t-T L(s))$, multiplied by the associated cost ( $C L(s))$, summed over all time periods later than the latest delivery time for shipments $s$.

$$
\sum_{s \in S}\left[\sum_{t=T L(s)+1}^{T}\left(\left[\sum_{l \in L E(D(s), t)} X S(s, l)\right] \cdot(t-T L(s)) \cdot C L(s)\right)\right]
$$




\section{Constraints}

- Shipment Conservation

Shipment Origin:

The flow of the shipments s that departs from $O(s)$ at the earliest pickup time is equal to the total size of the shipments s (Figure 7). The flow of shipments s that arrive at $O(s)$ at all other time other than the shipment's earliest pickup time is equal to zero (Figure 8).

$$
\sum_{l \in L B(O(s), T E(s))} X S(s, l)=W S(s) \quad \forall s \in S
$$

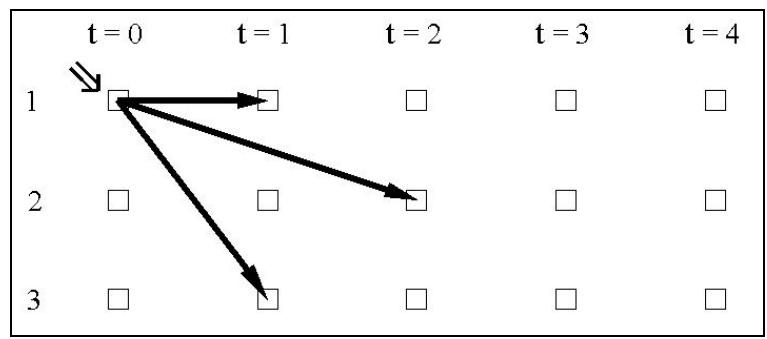

Figure 7 - Shipment conservation at shipment origin (constraint 16)

$$
\sum_{t=0}^{T} \sum_{l \in L E(O(s), t) \cap L R(t)} X S(s, l)=0 \quad \forall s \in S
$$

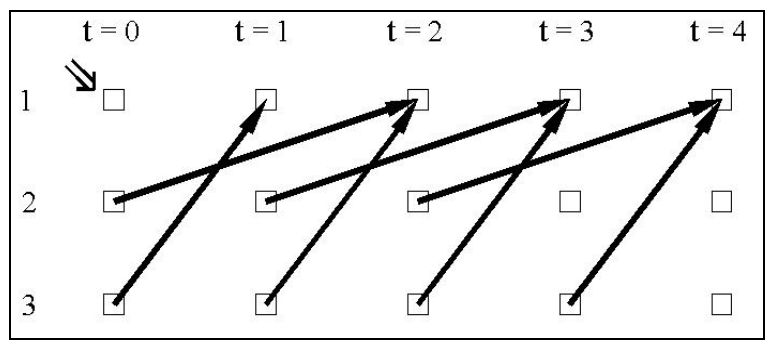

Figure 8 - Shipment conservation at shipment origin (constraint 17) 


\section{Shipment Destination:}

The Flow of the shipments $\mathrm{s}$ that arrive at $D(s)$ at all time periods is less than or equal to the total size of the shipments s (Figure 9). The flow of shipments $s$ that departs from $D(s)$ at all time periods is equal to zero (Figure 10).

$$
\sum_{t=0}^{T} \sum_{l \in L E(D(s), t)} X S(s, l) \leq W S(s) \quad \forall s \in S
$$

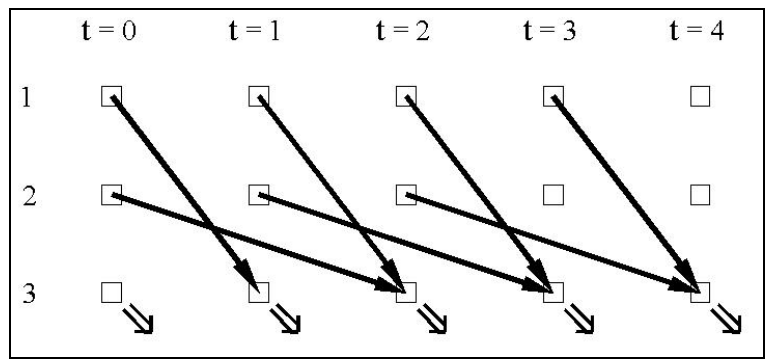

Figure 9 - Shipment conservation at shipment destination (constraint 18)

$$
\sum_{t=0}^{T} \sum_{l \in L B(D(s), t)} X S(s, l)=0 \quad \forall s \in S
$$

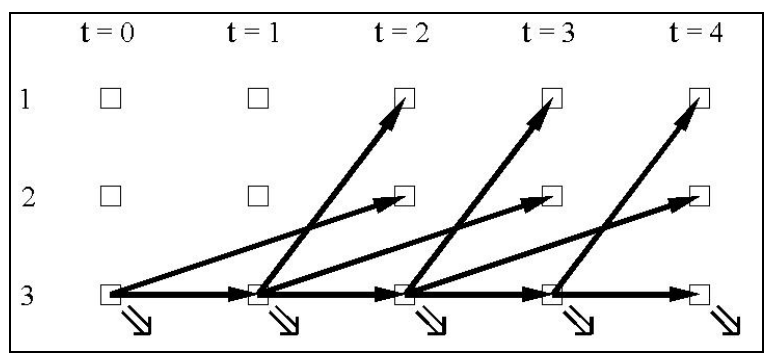

Figure 10 - Shipment conservation at shipment destination (constraint 19)

Other Nodes:

The flow of the shipments $s$ that arrives at every node in the time-space network is equal to the flow of the shipments $s$ that departs from that node (Figure 11).

$$
\begin{aligned}
& \sum_{l \in L E(n, t)} X S(s, l)=\sum_{l \in L B(n, t)} X S(s, l) \\
& \qquad \forall s \in S, \forall t \in[0, T], \forall n \in N-\{O(s), D(s)\}
\end{aligned}
$$




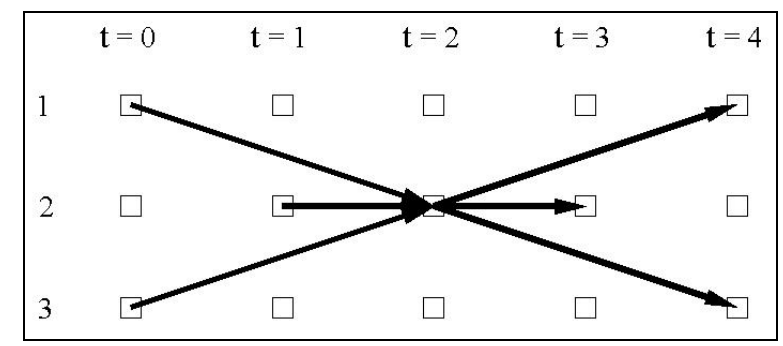

Figure 11 - Shipment conservation at all nodes (constraint 20)

- Shipment-Loaded Truck Connection:

The flow of all shipments over a link in the time-space network is less than or equal to the total truck capacity and greater than or equal to the total minimum fill rate on that link (Figure 12).

Truck Capacity:

$$
\sum_{s \in S} X S(s, l) \leq X F(l) . W F \quad \forall l \in L R(t), \forall t \in[0, T]
$$

Truck Minimum Fill Rate:

Note that, the following constraint is in form of "greater than or equal to" and is included in the mathematical formulation only to capture those instances where the dispatcher wants to impose some limitations on the minimum number of loads per each loaded truck. The constraint is added for the purpose of conducting some numerical experiments. Since this is a minimization problem, this constraint might even have a negative impact on the optimal solution.

$$
\sum_{s \in S} X S(s, l) \geq X F(l) . W E \quad \forall l \in L R(t), \forall t \in[0, T]
$$

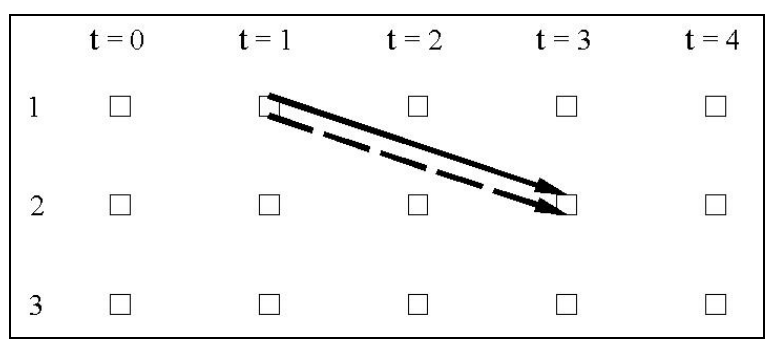

Figure 12 - Shipment-full (loaded) truck connection (constraints 21 and 22) 


\section{- Truck Conservation}

The total number of loaded and empty trucks that arrive at every node in the time-space network is equal to the total number of trucks that depart from that node (Figure 13).

$$
\begin{aligned}
S E(n, t)+\sum_{l \in L E(n, t)}[X F(l)+X E(l)]=\sum_{l \in L B(n, t)}[X F(l)+X E(l)] & \\
& \forall n \in N, \forall t \in[0, T]
\end{aligned}
$$

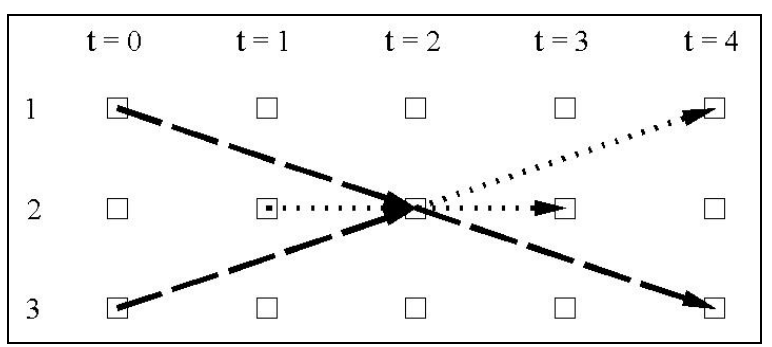

Figure 13 - Truck conservation (constraint 23)

- Non-Negativity

$X S(s, l) \geq 0$

$$
\forall s \in S, \forall l \in L R(t) \cup L W(t), \forall t \in[0, T]
$$

- Integrality

$X F(l)=0,1,2, \ldots$

$$
\begin{array}{r}
\forall l \in L R(t), \forall t \in[0, T] \\
\forall l \in L R(t) \cup L W(t), \forall t \in[0, T]
\end{array}
$$

$X E(l)=0,1,2, \ldots$ 


\section{Mathematical Formulation}

The final formulation of the model, which deals with the routing of shipments, loaded trucks, and empty trucks will have the following form:

$$
\begin{aligned}
& \text { Minimize } \\
& \sum_{t=0}^{T}\left[\sum_{l \in L R(t)}[X F(l) \cdot C F(l)]+\sum_{l \in L R(t)}[X E(l) \cdot C E(l)]\right]+\sum_{t=0}^{T} \sum_{l \in L R(t)} \sum_{s \in S}[X S(s, l) \cdot C H(s)]+ \\
& \left.\sum_{t=0}^{T} \sum_{l \in L W(t)} \sum_{s \in S}[X S(s, l) \cdot C W(s)]+\sum_{s \in S} \sum_{n \in N-\{D(s)\}}\left[\int_{l \in L E(n, T)} X S(s, l)\right) \cdot C N(s, n)\right]+ \\
& \sum_{s \in S}\left[\sum_{t=T L(s)+1}^{T}\left(\left[\sum_{l \in L E(D(s), t)} X S(s, l)\right] \cdot(t-T L(s)) \cdot C L(s)\right)\right]
\end{aligned}
$$

Subject to:

$$
\begin{array}{lc}
\sum_{l \in L B(O(s), T E(s))} X S(s, l)=W S(s) & \forall s \in S \\
\sum_{t=0}^{T} \sum_{l \in L E(O(s), t) \cap L R(t)} X S(s, l)=0 & \forall s \in S \\
\sum_{t=0}^{T} \sum_{l \in L E(D(s), t)} X S(s, l) \leq W S(s) & \forall s \in S \\
\sum_{t=0}^{T} \sum_{l \in L B(D(s), t)} X S(s, l)=0 & \forall s \in S \\
\sum_{l \in L E(n, t)} X S(s, l)=\sum_{l \in L B(n, t)} X S(s, l) & \forall s \in S, \forall t \in[0, T], \forall n \in N-\{O(s), D(s)\} \\
\sum_{s \in S} X S(s, l) \leq X F(l) . W F & \forall l \in L R(t), \forall t \in[0, T] \\
S E(n, t)+\sum_{l \in L E(n, t)}[X F(l)+X E(l)]=\sum_{l \in L B(n, t)}[X F(l)+X E(l)] \quad \forall n \in N, \forall t \in[0, T] & \forall s \in S, \forall l \in L R(t) \cup L W(t), \forall t \in[0, T] \\
X S(s, l) \geq 0 & \forall l \in L R(t), \forall t \in[0, T] \\
X F(l)=0,1,2, \ldots & \forall l \in L R(t) \cup L W(t), \forall t \in[0, T] \\
X E(l)=0,1,2, \ldots & \forall l \in
\end{array}
$$




\section{Extension 1: Non-homogeneous Fleet}

In this study, fleet can be treated as a commodity in the MCNF model. In the previous sections, the mathematical model was developed with the assumption of having a homogeneous fleet. As the first extension to the original mathematical model, fleet is assumed to be non-homogeneous. To capture this assumption, one commodity is added to the system for each type of trucks. This section presents the minor modifications that are required in order to deal with the non-homogeneous fleet assumption:

Trucks

$$
\begin{array}{ll}
K & =\text { Set of all types of trucks } k \in K \\
W F(k) & =\text { Capacity of a type } k \text { truck } \\
W E(k) & =\text { Minimum fill rate of a type } k \text { truck } \\
S E(k, n, t) & =\text { Supply of empty type } k \text { trucks on node } n \in N \text { at time period } t
\end{array}
$$

Cost Functions

$$
\begin{aligned}
& C F(k, l) \quad=\text { Full (loaded) type } k \text { truck routing cost over link } l \in L R(t) \\
& C E(k, l) \quad=\text { Empty type } k \text { truck routing cost over link } l \in L R(t)
\end{aligned}
$$

Decision Variables

$$
\begin{aligned}
& X F(k, l)=\text { Flow of full (loaded) type } k \text { trucks on link } l \in L R(t) \\
& X E(k, l)=\text { Flow of empty type } k \text { trucks on link } l \in L R(t)
\end{aligned}
$$

\section{Routing Costs}

$$
\sum_{k \in K} \sum_{t=0}^{T}\left[\sum_{l \in L R(t)}[X F(k, l) . C F(k, l)]+\sum_{l \in L R(t)}[X E(k, l) \cdot C E(k, l)]\right]
$$

Truck Capacity

$$
\sum_{s \in S} X S(s, l) \leq \sum_{k \in K} X F(k, l) . W F(k) \quad \forall l \in L R(t), \forall t \in[0, T]
$$


Truck Minimum Fill Rate

$$
\sum_{s \in S} X S(s, l) \geq \sum_{k \in K} X F(k, l) . W E(k) \quad \forall l \in L R(t), \forall t \in[0, T]
$$

Truck Conservation

$$
\begin{array}{r}
S E(k, n, t)+\sum_{l \in L E(n, t)}[X F(k, l)+X E(k, l)]=\sum_{l \in L B(n, t)}[X F(k, l)+X E(k, l)] \\
\forall n \in N, \forall t \in[0, T], \forall k \in K \quad(30)
\end{array}
$$

Integrality

$X F(k, l)=0,1,2, \ldots \quad \forall k \in K, \forall l \in L R(t), \forall t \in[0, T]$

$X E(k, l)=0,1,2, \ldots \quad \forall k \in K, \forall l \in L R(t) \cup L W(t), \forall t \in[0, T]$

In summary, the routing costs of the homogeneous problem are replaced with the routing costs of the non-homogeneous fleet. Truck capacity, minimum fill rate, conservation and integrality constraints are also modified to capture the nonhomogeneous fleet assumption. All the other components of the objective function and the constraints that are associated with shipments remain unchanged. The final formulation of the mathematical model considering the non-homogeneous fleet assumption is as follows:

Minimize (27) + (12) + (13) + (14) + (15)

Subject to constraints (16) to (20), (24) and (28) to (32) 


\section{Extension 2: Driver Routing}

An important dimension of the LTL trucking operations is driver management. This is the second extension to the original mathematical model. The original model is modified by considering the driver routing and scheduling problem, combined with truck/shipment routing and scheduling problem.

Driver work rules limit the length of time a driver can work. There are limitations on the number of work hours during a shift. Also, drivers cannot be on the road without having a rest period between each two shifts. To incorporate these constraints it is assumed that the LTL network is designed in a way that the length of each link is not longer than what a driver can driver during a work shift.

Drivers in LTL trucking may spend a couple of weeks away from home (their base terminal). Most carriers try to return a driver home earlier. In order to take this important factor into account, a penalty cost is defined that is associated with keeping drivers away from home for a long period of time.

This section introduces the required additions to assumptions, notations, objective function and constraints of the original mathematical formulation in order to model the combined driver/truck/shipment routing and scheduling problem. Note that, the proposed extension would increase the complexity of the problem and solving the resulting problem is beyond the focus of this study. However, limited numerical experiments were conducted considering the driver routing extension and the results are reported in Chapter 5.

By adding the following assumptions, notations, additional cost functions and constrains, the characteristics of the driver routing problem will be captured:

Assumption

$T T(l)<$ Maximum driver duty time per day

Drivers

$D \quad=$ Set of all drivers $d \in D$

$H(d)=$ Base (home) terminal for driver $d \in D$

$T(d)=$ Maximum number of duty time periods for driver $d \in D$ 
$S D(d, n, t)=1$ when driver $d$ is at terminal $n$ at time $t, 0$ otherwise

Cost Function

$C D(d, n)=$ Penalty cost associated with keeping driver $d$ at terminal $n$ (any terminal other than home) at the end of horizon

Decision Variable

$X D(d, l)=1$ if driver $d$ works/rests on link $l$, and 0 otherwise

Routing Costs

$\left.\sum_{d \in D} \sum_{n \in N-\{H(d)\}}\left[\left[\sum_{l \in L E(n, T)} X D(d, l)\right] \cdot C D(d, n)\right]\right]$

Driver-Truck Connection

$$
\sum_{d \in D} X D(d, l)=X E(l)+X F(l) \quad \forall l \in L R(t), \forall t \in[0, T]
$$

Driver Conservation

$$
\begin{aligned}
& S D(d, n, t)+\sum_{l \in L E(n, t)} X D(d, l)=\sum_{l \in L B(n, t)} X D(d, l) \\
& \quad \forall d \in D, \forall n \in N, \forall t \in[0, T]
\end{aligned}
$$

Driver Work Time Regulation

$$
\sum_{l \in L R(t)} X D(d, l)+\sum_{l \in L R(t+T(d))} X D(d, l) \leq 1 \quad \forall d \in D, \forall t \in[0, T]
$$

Integrality

$X D(d, l)=0,1$

$$
\forall d \in D, \forall l \in L R(t), \forall t \in[0, T]
$$

In summary, the driver related routing costs are added to the objective function of the original problem. Additional constraints are added to capture the driver-truck connection, driver conservation, driver work time regulation and 
integrality. All other components of the objective function and the constraints remain unchanged. The final formulation of the mathematical model considering the driver routing problem is as follows:

Minimize (11) + (12) + (13) + (14) + (15) + (33)

Subject to constraints (16) to (26) and (34) to (37) 


\section{Extension 3: Early Delivery Costs}

This section introduces a new addition to the operational costs. The main goal is to capture the situations where a part of a shipment arrives at the destination terminal, while the rest is still on its way. In these cases the customer doesn't accept loads in separate batches and wants them to be delivered all at once. Hence, the carrier has to provide storage to keep that part of the demand that is delivered earlier. This section presents the additional cost element, which is called "early delivery cost". The results of sensitivity analyses are conducted to show how changes in early delivery cost factors may affect the system performance. He results are reported in Chapter 5.

\section{Cost Functions}

$$
C P(s)=\text { Penalty (Waiting) cost for partial delivery for shipment } s \in S
$$

\section{Early Delivery Cost}

$$
\sum_{s \in S}\left[\sum_{t=T L(s)+1}^{T}\left(\left[\sum_{l \in L E(D(s), t)} X S(s, l)-\sum_{l \in L E(D(s), t-1)} X S(s, l)\right] .(t-T L(s)) \cdot C L(s)\right)\right]
$$

In summary, one additional component is added to the objective function that covers the early delivery costs. Other parts of the original mathematical model remain unchanged. The final formulation of the mathematical model considering the additional waiting costs is as follows:

Minimize (11) $+(12)+(13)+(14)+(38)$

Subject to constraints (16) to (26) 
This section introduced the mathematical formulation that is developed for the dynamic shipment and vehicle routing and scheduling problem for LTL trucking operation. The original mathematical formulation can be modified to include other dimensions in LTL trucking operation. Three extensions were presented in this Chapter with details on major modification that is required to capture the nonhomogeneous fleet, the driver routing and the additional waiting costs. These extensions can be considered individually or combined. Considering these extensions would result in a much more realistic optimization model. However, it would also increase the complexity of the mathematical problem, and solving the resulting problem is beyond the focus of this study. Limited numerical experiments are conducted considering these extensions and the results are reported in Chapters 5 and 7. The main goal of this presentation is to pave the road for the future research efforts. 


\section{Chapter 4: Decision Making Procedures}

This Chapter presents a procedure that is proposed to make acceptance/rejection decisions for LTL motor carriers. The discussion is followed by a presentation of a decision support application which is developed based on the proposed decision making procedure.

As discussed earlier it is assumed that strategic and tactical level decisions have been made already which means the LTL service network structure (terminal location, direct services, etc.) and fleet characteristics (fleet size and locations) are known. Furthermore, there are parameters related to the length of time periods, duration of the planning horizon, trucks (capacity, minimum fill rate, etc.), and shipments (size, origin, destination, etc.) that are used as additional inputs for the proposed decision making procedure.

Figure 14 shows the conceptual framework of the decision making procedure. The system real-time information is available either through simulation or the real data from the field. At the end of the time horizon or each time a request arrives, the mathematical formulation of the problem is generated considering the updated empty truck locations and shipments information. Then the problem is solved either by CPLEX, or by heuristic solution methods as needed. In this study three heuristic solution methods are introduced as an alternative to the available exact solution methods (i.e. CPLEX). The detail discussion on these proposed algorithms and their performance are provided in the next Chapters.

After solving the problem, the performance of the new solution is analyzed and the effects of accepting the new requests are examined based on the acceptance criteria. LTL trucking is similar to a manufacturing firm in which the input is the LTL network, fleet and demand and the output is the delivery service. The marginal cost is the increase or decrease in the operational cost as the result of one more unit of output. 


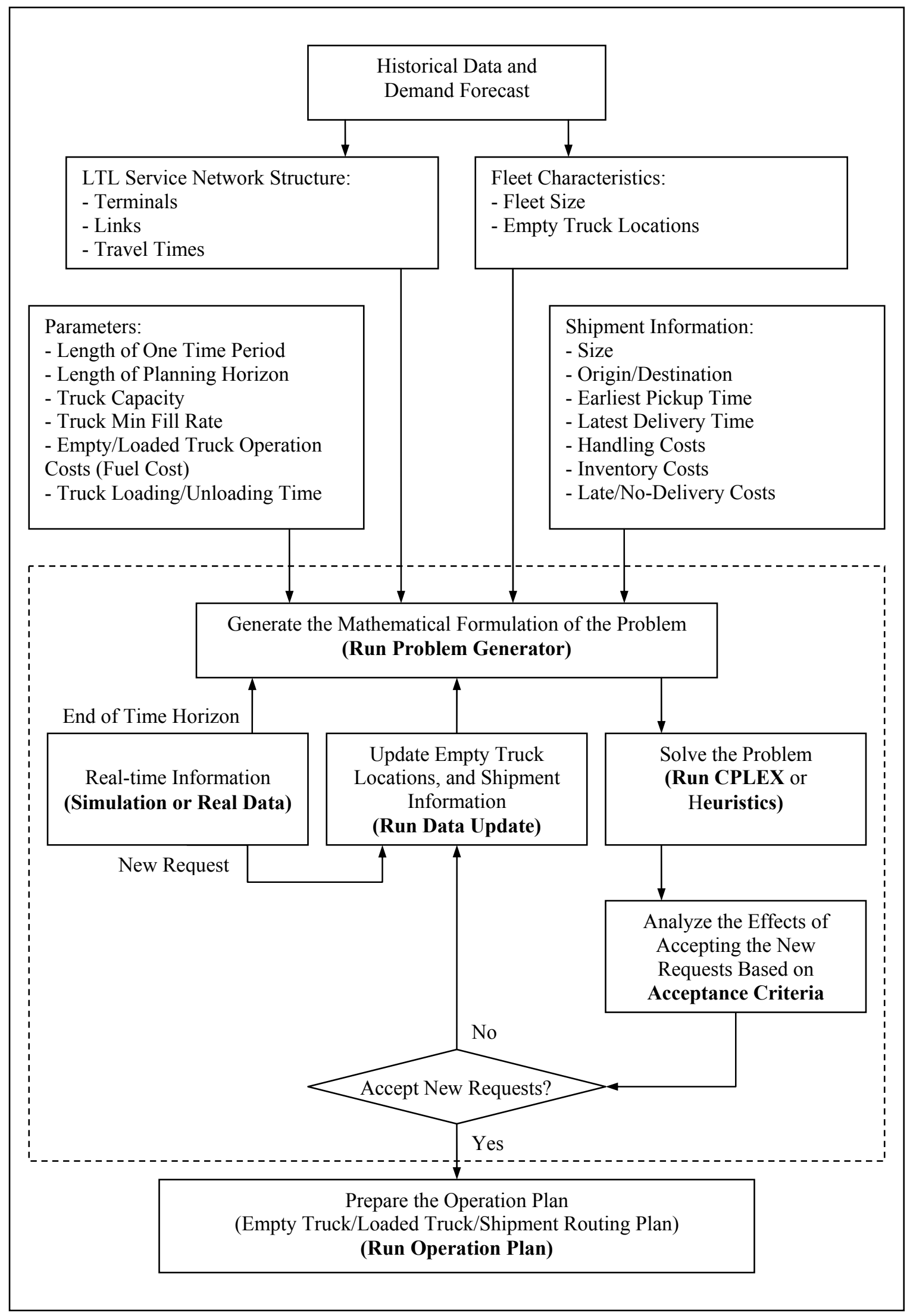

Figure 14 - Decision making procedure 
Like most manufacturing firms, in LTL trucking, the marginal costs decrease as the volume of output increases (accepting more shipments for delivery) due to economies of scale. This is achieved by shipment consolidation that leads to an efficient use of supply. On the other hand, less efficient input can cause diseconomies of scale which increase the marginal cost. Marginal cost can be used for pricing the LTL transportation services and also as a measure to evaluate the effects of acceptance/rejection of a shipment request. Based on these acceptance criteria the carrier may offer a price that makes a shipment profitable. Therefore, the rates that are offered by the company for delivery service can be a function of shipment characteristics.

Based on the acceptance criteria, the decision is being made either to accept the load or to reject it. If the load is accepted the operation plan will be prepared and sent to the field. Otherwise, no change is applied to the current operation plan.

Normally, the customers shop around and get quotes from a number of trucking companies. They read customer reviews on those companies, compare their rates and make their final decision. Considering the customer behavior, and based on the results obtained from the decision making procedure the dispatcher may pick one of these options:

- If the load is economical, it is accepted and the regular rate can be offered

- If the load is economical, it is accepted and a rate lower than regular rate can be offered to the customer based on the marginal cost of the shipment delivery

- If the load is not economical, the dispatcher can wait for the future shipments requests that may affect the current decision

- If the load is not economical, a rate higher than regular rate can be offered to the customer to compensate the additional costs of shipment delivery In other words, this procedure suggested that the carrier never provides information regarding the rejection of a load to a customer. Instead, either a rate higher than regular rate is offered, or the dispatcher waits for future requests that may bring a better opportunity for consolidation for the currently unaccepted loads. For large-size problems and under high demand for LTL shipments, it is not practical to 
use CPLEX. One way to resolve this issue is to solve the MIP using a heuristic approach that is the main focus of Chapter 6.

\section{Fast-Acceptance Techniques}

In this section, a variation of the decision making procedure is proposed that can be used under high demand condition to increase the ability of handling the requests when solving the optimization problem is computationally expensive. By applying these methods the dispatcher can avoid solving the main MIP program each time a new shipment arrives. These techniques do not provide the best solution but they act fast in the dynamic environment by accepting the most profitable loads.

One way to speed up the decision-making process is to identify the shipments that are compatible with the current operation plan. Based on the proposed procedure (Figure 15) each time a new request arrives the acceptance techniques are used to check if the new request can be inserted into the current solution without any further changes. If the load gets accepted the operation plan will be updated. Those requests that cannot be picked at this stage will have another chance to get selected. Such requests are all added to a pool of unaccepted requests. When the pool reaches its capacity the re-optimization process will proceed to make the decision to accept/reject the loads.

In order to check the compatibility of the new shipments with the current solution two different algorithms are proposed. In both approaches, a new MCNF problem is generated based on the new shipments and the current solution. The size of this problem is smaller compare to the original problem and it can be solved much faster using the exact or heuristic methods.

- Technique 1 (Fixed Truck Routing)

In the preparation step, the excess capacity of all links is calculated. To calculate this value, the total number of shipments that go through a link is subtracted from the total available capacity on the empty and half-loaded trucks on the same link based on the current routing plan.

In the new MCNF problem, only the new shipments are considered. The objective function consists of all costs related to shipments, which includes 
components (12), (13), (14) and (15) in the objective function of the original problem. The goal is to minimize these costs subject to constraints (16)-(20) and (24). Furthermore, the sum of the new shipments that routed over each link must be less than or equal to the total excess capacity of that link.

The decision variables in this new MCNF problem are only the volumes of the new shipments on each link. After solving the problem and finding the value of these volumes, the volumes of empty and loaded trucks on each link are updated. Obviously, this problem is much smaller than the original problem, in terms of number of variables and constraints. By solving this problem, the marginal cost of accepting new shipments can be estimated and decisions can be made on the acceptance or rejection of the new loads.

- Technique 2 (Adaptive Truck Routing)

This technique is similar to the $1^{\text {st }}$ fast acceptance technique in terms of their ultimate goal, which is using the excess capacity in the current routing plan to satisfy the new demand. However, unlike the first technique, in this approach all shipments are considered; the new shipments as well as the already accepted ones. The current shipment plan is used and the volume of old shipments on the network assumed to be fixed. This means that the algorithm make all accepted loads to go through the same path that the current routing plan suggested. By applying this shipment partitioning, a large number of decision variables are removed from the problem and the size of the resulting MCNF problem would be much smaller than the original problem.

The decision variables in this new MCNF problem are the volumes of the new shipments and empty/loaded trucks on each link. After solving the problem and finding the value of these volumes, the marginal cost of accepting new shipments can be estimated and decisions can be made on the acceptance or rejection of the new loads. In this approach the possible changes in the volume of empty and loaded trucks are taken into account. Therefore, the solution provided by this approach is expected to have better quality compared to the results that are obtained from the first technique. 


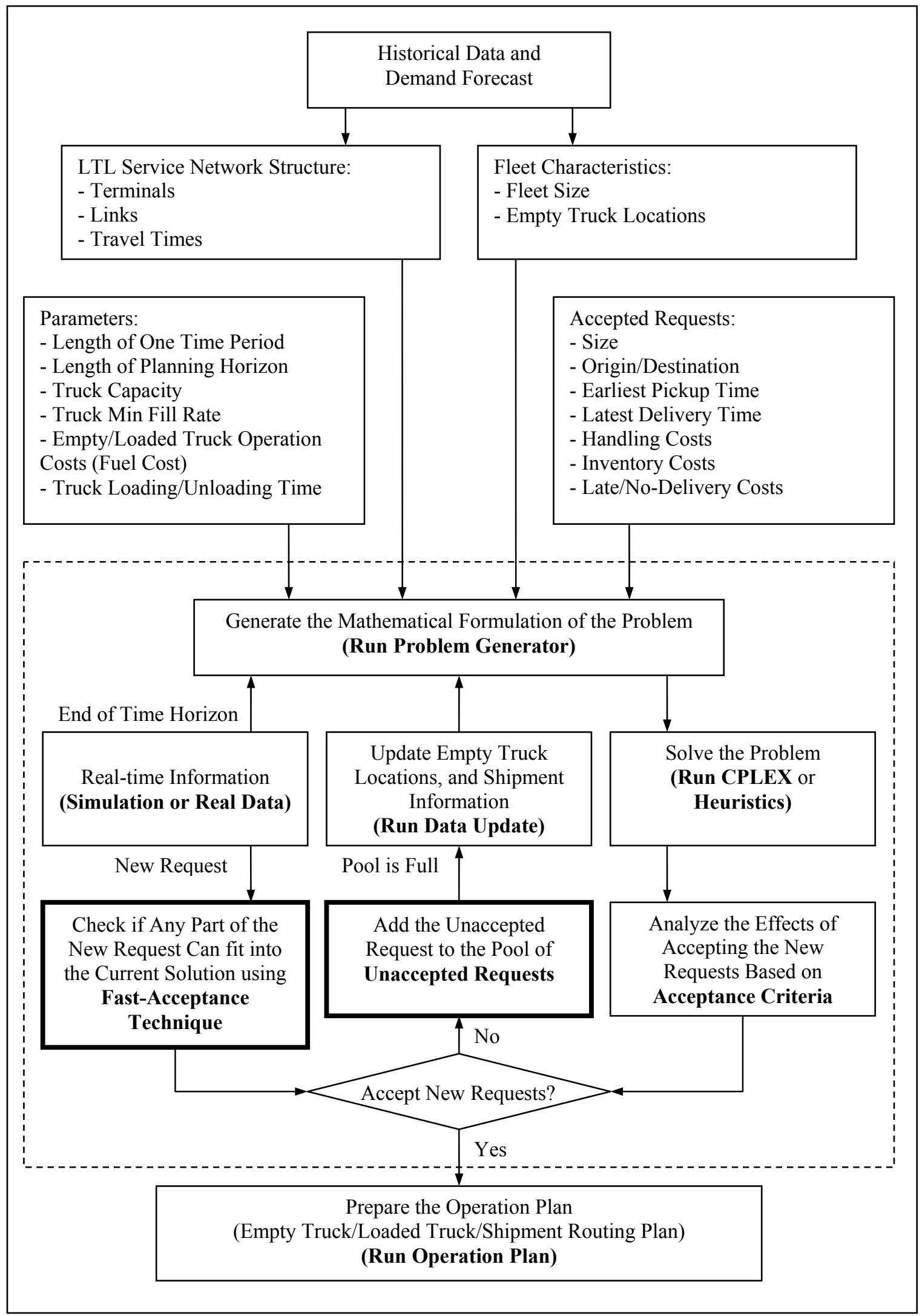

Figure 15 - Decision making procedure (with Fast-Acceptance Technique) 


\section{Decision Making Application}

A dynamic decision making application is developed, based on the original decision making procedure. The application which is shown in Figure 16 is a decision support system that can be used to analyze the effects of accepting different combination of shipments. This is an interactive tool, which has been developed using Visual Basic, and consists of the following components:

- $\quad$ Shipment Management Panel: This panel is used for data entry and managing the list of shipments. When a new request arrives the dispatcher enters the shipment and its related information to the list. This provides a tool for manual and automatic dispatching. Dispatcher can select any combination of shipments, add them to the list or remove them from the list prior to solving the optimization problem. On the top of this panel, the list of accepted shipments is shown.

- Decision Making Panel: In the next step the dispatcher generates the optimization problem by clicking on the "Generate Problem" button. This command runs the Problem Generator program that uses the updated information for trucks and shipments and creates the mathematical model for the problem. The problem characteristics are summarized by the application and can be used to double-check the inputs. After the problem is solved (by CPLEX or heuristics), a second C program (Operation Plan) prepares the performance measures and shipments routing/dispatch plans that are available for dispatchers to make the final decision. Figures 17, 18, 19, and 20 are examples of performance measures, shipment routing, shipment dispatch, and truck dispatch that all are generated by "Operation Plan" based the solution of the mathematical problem. To capture the dynamic nature of the system a third program is also developed in C called "Data Update". This program operates in the background and updates the empty truck locations and shipment information at each stage of the decisionmaking process. 


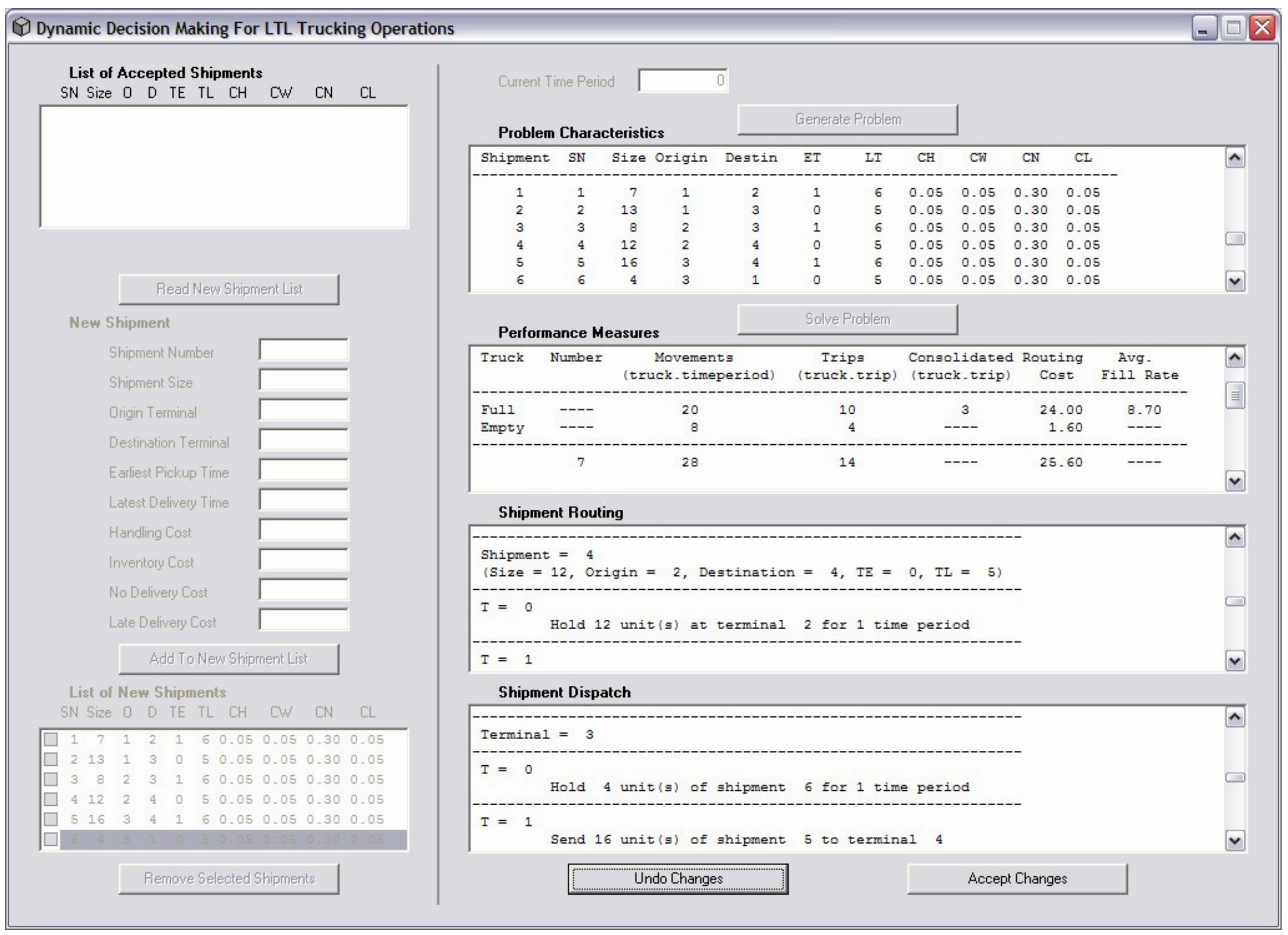

Figure 16 - Dynamic decision making application for LTL trucking operations 


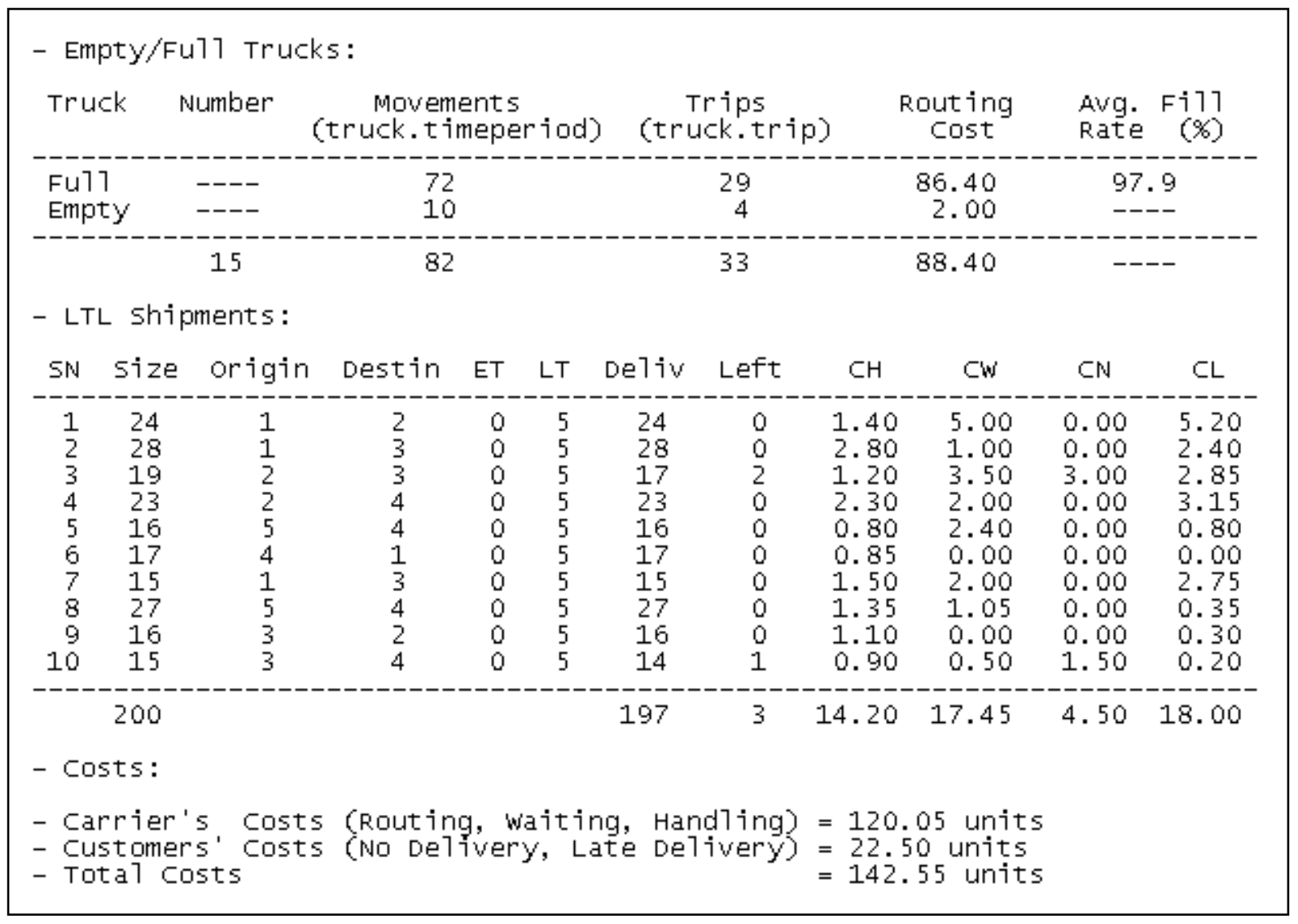

Figure 17 - LTL application output: Performance measures 


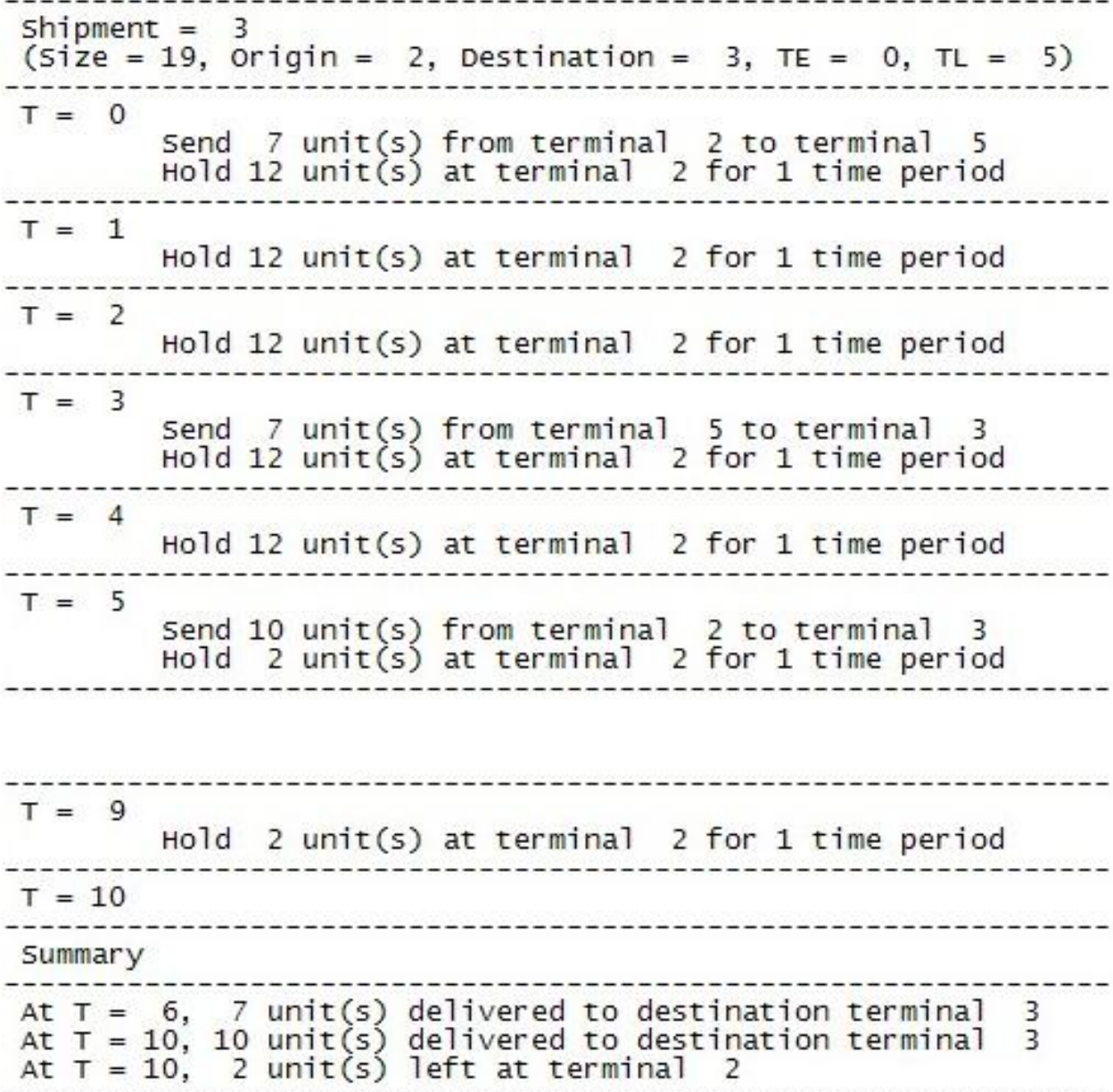

Figure 18 - LTL application output: Shipment routing plan 


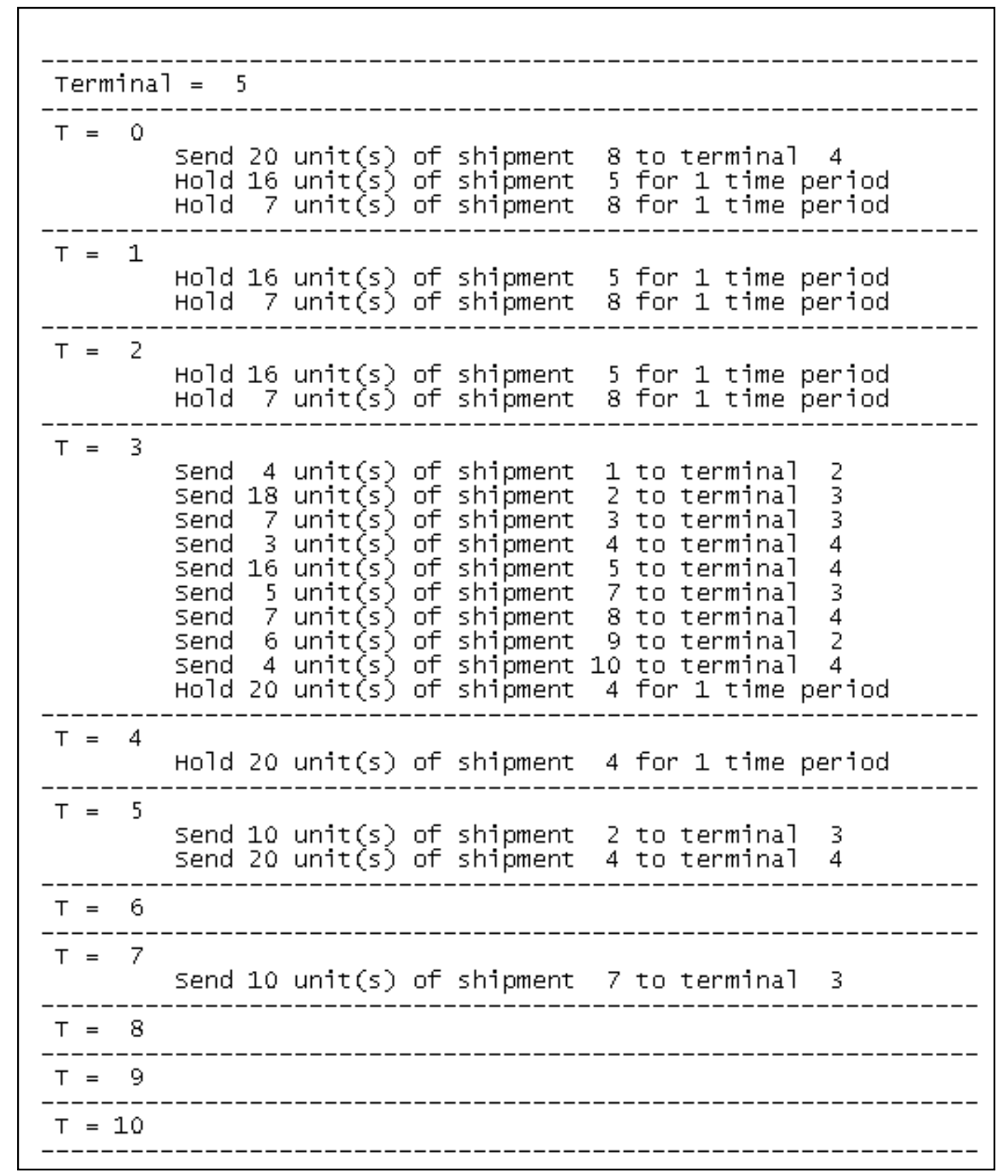

Figure 19 - LTL application output: Shipment dispatch plan 


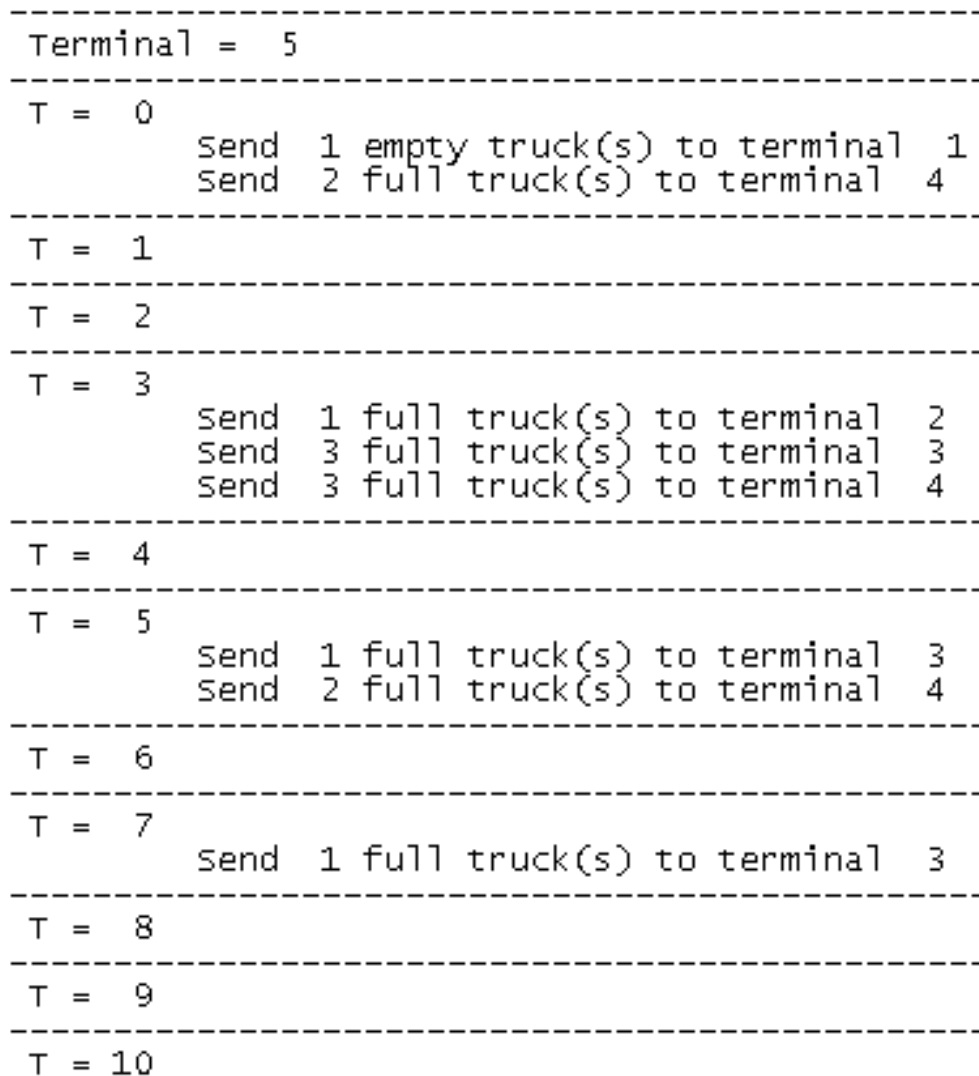

Figure 20 - LTL application output: Truck dispatch plan 
As discussed earlier, the main goal in dynamic decision making is to reduce the response time. The time-consuming components of the process are:

- Data management and handling the input/output files

- Solving the optimization problem

This section presented the decision support application that was developed in order to facilitate the data management and handling the input/output files. The user takes the advantage of shipment management panel, tries different load combination and updates the database without working with the actual data files. The decision support tool provides the required connection between "Problem Generator", CPLEX (or other problem solver program), "Data Update" and "Operation Plan" programs. The user is capable of evaluating the impacts of different decision making techniques on the system performance. The proposed application can be used both in simulation runs and the literature-reported operation. 


\section{Chapter 5: Preliminary Numerical Experiments}

The results of two numerical experiments are reported in this Chapter. The first numerical test is on a small-size 5-terminal network in order to check the accuracy of the mathematical formulation as well as the computer programs that have been developed to generate the problem and manage the solution results. The second set of numerical experiments is conducted on a 10-terminal network to study the system behavior and sensitivity of the solution with respect to changes in the contributing factors.

The American Trucking Association Foundation (ATA) uses the following classification for motor carriers based on the annual gross operating revenue [6]:

- Class I carriers are those receiving annual gross operating revenues (including interstate and intrastate) of $\$ 10$ million or more from operations.

- Class II carriers are those receiving annual gross operating revenues (including interstate and intrastate) of $\$ 3$ million to $\$ 9,999,999$ from operations.

- Class III carriers are those receiving annual gross operating revenues (including interstate and intrastate) of less than \$3 million from operations.

The collection of trucking company financial and operating statistics (F\&OS) data is a mandatory program managed by the Bureau of Transportation Statistics (BTS). Motor carriers which have gross annual operating revenue of $\$ 3$ million or more are required to report annually, while carriers with revenues of $\$ 10$ million or more must also file four quarterly reports each year.

Auto carriers are one class of motor carriers that transport vehicles throughout the United States for individuals, car dealers, automobile manufacturers, vehicle leasing companies and moving companies who are involved in the auto transport of household goods as well as car moving. Tables 2 to 6 show lists of class I and class II 
auto carriers, their base state, operating revenue and expenses, total miles, tons and ton-miles based on their annual reports to DOT for 5 years 1999 through 2003 [6].

Auto carriers are similar to LTL trucking companies in terms of network structure, size of shipments and type of operations, so this type of carriers are selected to perform the numerical experiments in this study. Auto carriers operate using huband-spoke networks. Customers can place a request for service either online or by calling the company.

Auto carrier asks for three types of information: Customer information (Name, and contact information), Vehicle information (Year, Make, Model, Type, Color, and Operating Condition) and Shipment Information (Origin and Destination).

After receiving the information the carrier contacts customer with a quote price within a couple of hours. They don't guarantee the pick-up date and time but they typically say they pickup any vehicle within two to three days. They also say that their competitors typically require 1-3 weeks to set up a pickup time! There are different types of equipments that auto carriers use to haul the cars [82]. The only type of car carrier that is recognized as multiple car carrier by the DOT are "Stingers", the same type used to transport new vehicles from manufacturing plant to dealerships (Figure 21). These trailers can haul up to 10 to 12 cars.

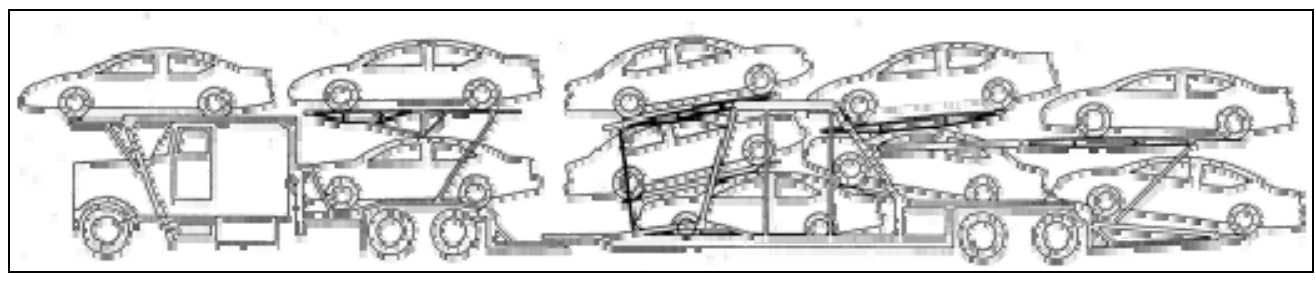

Figure 21 - Multiple-car carrier 
Table 2 - Financial and operating statistics of class I, and class II auto carriers (1999)

\begin{tabular}{cccccccc}
\hline \# & $\begin{array}{c}\text { Motor } \\
\text { Carrier } \\
\#\end{array}$ & State & $\begin{array}{c}\text { Total } \\
\text { Operational } \\
\text { Revenue } \\
\text { (\$ million) }\end{array}$ & $\begin{array}{c}\text { Total } \\
\text { Operational } \\
\text { Expenses } \\
\text { (\$ million) }\end{array}$ & $\begin{array}{c}\text { Miles } \\
\text { (million) }\end{array}$ & $\begin{array}{c}\text { Tons } \\
\text { (thousand) }\end{array}$ & $\begin{array}{c}\text { Ton- } \\
\text { Miles } \\
\text { (million) }\end{array}$ \\
\hline 1 & 71902 & Missouri & --- & 107.7 & 38.0 & --- & 442.4 \\
2 & 112391 & California & 103.9 & 100.8 & 33.0 & 3601.6 & 330.3 \\
3 & 26396 & Montana & --- & 76.1 & 45.1 & --- & --- \\
4 & 177129 & Colorado & --- & 74.8 & 30.7 & --- & 265.6 \\
5 & 215978 & Missouri & 54.7 & 53.0 & 14.0 & 1450.2 & 145.1 \\
6 & 208434 & Georgia & 35.1 & 35.5 & 11.9 & 505.1 & 78.2 \\
7 & 170323 & Florida & 31.9 & 31.4 & --- & --- & --- \\
8 & 133993 & Alabama & 24.7 & 24.4 & 11.6 & --- & --- \\
9 & 134614 & Washington & --- & 19.7 & 8.7 & 152.4 & --- \\
10 & 224791 & Florida & 10.9 & 10.2 & 4.2 & --- & --- \\
11 & 226461 & Texas & 13.6 & 9.5 & 4.5 & 190.8 & 68.7 \\
12 & 174971 & California & --- & 9.4 & --- & --- & 48.2 \\
13 & 153385 & Colorado & --- & 8.8 & 3.7 & --- & 50.9 \\
14 & 117380 & Nevada & --- & 8.0 & 1.1 & --- & --- \\
15 & 148860 & Maryland & 7.9 & 7.8 & 3.2 & 130.0 & --- \\
16 & 265499 & Indiana & --- & 5.6 & --- & --- & --- \\
17 & 257561 & Oklahoma & 6.0 & 4.8 & 2.0 & 218.4 & 18.7 \\
18 & 163921 & Florida & --- & 4.7 & 2.2 & --- & --- \\
\hline
\end{tabular}


Table 3 - Financial and operating statistics of class I, and class II auto carriers (2000)

\begin{tabular}{cccccccc}
\hline Motor & $\begin{array}{c}\text { Carrier } \\
\#\end{array}$ & State & $\begin{array}{c}\text { Total } \\
\text { Operational } \\
\text { Revenue } \\
\text { (\$ million) }\end{array}$ & $\begin{array}{c}\text { Total } \\
\text { Operational } \\
\text { Expenses } \\
\text { (\$ million) }\end{array}$ & $\begin{array}{c}\text { Miles } \\
\text { (million) }\end{array}$ & $\begin{array}{c}\text { Tons } \\
\text { (thousand) }\end{array}$ & $\begin{array}{c}\text { Ton- } \\
\text { Miles } \\
\text { (million) }\end{array}$ \\
\hline 1 & 213250 & Georgia & 794.8 & 791.7 & 241.1 & 10839.8 & 2460.6 \\
2 & 42537 & Illinois & --- & 202.2 & 75.7 & 221.2 & 911.7 \\
3 & 177129 & Colorado & 126.5 & 120.0 & 54.9 & 700.4 & 448.0 \\
4 & 71902 & Missouri & --- & 116.9 & 41.0 & --- & 1092.0 \\
5 & 112391 & California & 112.5 & 104.7 & 32.4 & 3563.0 & 319.6 \\
6 & 103993 & Indiana & 93.2 & 102.7 & 71.9 & 1121.5 & 476.4 \\
7 & 215978 & Missouri & --- & 58.4 & 15.6 & --- & 271.2 \\
8 & 155097 & Missouri & --- & 41.2 & --- & --- & --- \\
9 & 133993 & Alabama & --- & 32.0 & 11.9 & --- & --- \\
10 & 248649 & Indiana & --- & 23.1 & 32.4 & 270.0 & 0.3 \\
11 & 134614 & Washington & 18.9 & 22.9 & 1.0 & 182.1 & --- \\
12 & 170323 & Florida & --- & 22.2 & 18.0 & --- & --- \\
13 & 226461 & Texas & --- & 14.2 & 6.3 & --- & 100.9 \\
14 & 153385 & Colorado & 8.9 & 12.1 & 4.2 & 121.7 & 57.6 \\
15 & 36824 & Texas & 11.5 & 11.3 & 5.0 & --- & --- \\
16 & 174971 & California & --- & 10.1 & --- & --- & 53.3 \\
17 & 98938 & Massachusetts & 9.0 & 9.1 & 2.5 & 60.4 & 1.3 \\
18 & 117380 & Nevada & --- & 8.9 & 1.2 & --- & --- \\
19 & 148860 & Maryland & --- & 8.8 & 3.7 & --- & --- \\
20 & 193564 & Texas & --- & 8.4 & --- & --- & --- \\
21 & 143453 & New Jersey & 6.9 & 6.8 & 2.6 & 11.5 & 5.9 \\
22 & 164021 & Indiana & --- & 5.7 & 6.6 & 102.5 & 71.9 \\
23 & 250710 & Indiana & --- & 5.2 & --- & --- & --- \\
24 & 224791 & Florida & --- & 5.1 & --- & --- & --- \\
\hline & & & & & & & \\
\hline
\end{tabular}


Table 4 - Financial and operating statistics of class I, and class II auto carriers (2001)

\begin{tabular}{|c|c|c|c|c|c|c|c|}
\hline$\#$ & $\begin{array}{c}\text { Motor } \\
\text { Carrier } \\
\#\end{array}$ & State & $\begin{array}{c}\text { Total } \\
\text { Operational } \\
\text { Revenue } \\
\text { (\$ million) } \\
\end{array}$ & $\begin{array}{c}\text { Total } \\
\text { Operational } \\
\text { Expenses } \\
\text { (\$ million) } \\
\end{array}$ & $\begin{array}{c}\text { Miles } \\
\text { (million) }\end{array}$ & $\begin{array}{c}\text { Tons } \\
\text { (thousand) }\end{array}$ & $\begin{array}{c}\text { Ton- } \\
\text { Miles } \\
\text { (million) }\end{array}$ \\
\hline 1 & 213250 & Georgia & --- & 727.4 & --- & --- & --- \\
\hline 2 & 42537 & Illinois & --- & 177.1 & 65.0 & 3508.3 & 857.7 \\
\hline 3 & 71902 & Missouri & 103.0 & 114.8 & 40.0 & 3019.5 & 880.5 \\
\hline 4 & 103993 & Indiana & --- & 95.4 & 63.8 & --- & 477.2 \\
\hline 5 & 112391 & California & 100.0 & 90.4 & 28.1 & 3200.4 & 277.0 \\
\hline 6 & 177129 & Colorado & --- & 68.1 & --- & --- & --- \\
\hline 7 & 215978 & Missouri & 62.6 & 59.5 & 16.2 & 1613.2 & 272.0 \\
\hline 8 & 155097 & Missouri & --- & 43.6 & --- & --- & --- \\
\hline 9 & 133993 & Alabama & --- & 37.8 & 10.5 & --- & --- \\
\hline 10 & 170323 & Florida & --- & 22.2 & 8.2 & --- & --- \\
\hline 11 & 134614 & Washington & --- & 22.0 & 9.5 & --- & --- \\
\hline 12 & 248649 & Indiana & --- & 21.1 & 26.8 & --- & --- \\
\hline 13 & 226461 & Texas & --- & 15.6 & 8.1 & 266.1 & 120.0 \\
\hline 14 & 36824 & Texas & 12.4 & 12.4 & 5.8 & --- & --- \\
\hline 15 & 210561 & Arizona & --- & 11.8 & 2.9 & --- & --- \\
\hline 16 & 380001 & Florida & --- & 10.6 & 3.5 & 196.9 & 78.9 \\
\hline 17 & 153385 & Colorado & 9.5 & 10.2 & 4.3 & 241.3 & 58.0 \\
\hline 18 & 174127 & Missouri & --- & 10.2 & --- & --- & --- \\
\hline 19 & 363019 & Florida & --- & 9.8 & --- & --- & --- \\
\hline 20 & 148860 & Maryland & --- & 9.2 & 3.7 & --- & --- \\
\hline 21 & 255969 & Michigan & --- & 9.1 & 4.0 & --- & 37.0 \\
\hline 22 & 98938 & Massachusetts & --- & 8.8 & 2.4 & 56.4 & --- \\
\hline 23 & 117380 & Nevada & --- & 8.3 & 1.2 & --- & --- \\
\hline 24 & 174971 & California & --- & 8.2 & --- & --- & --- \\
\hline 25 & 106205 & New York & --- & 7.8 & 1.1 & --- & --- \\
\hline 26 & 193564 & Texas & 6.8 & 6.7 & --- & --- & --- \\
\hline 27 & 143453 & New Jersey & --- & 6.1 & 2.7 & --- & 5.4 \\
\hline 28 & 246636 & Kansas & --- & 5.7 & 4.8 & --- & --- \\
\hline 29 & 164021 & Indiana & --- & 5.2 & 8.3 & --- & 113.9 \\
\hline 30 & 250710 & Indiana & --- & 4.3 & --- & --- & --- \\
\hline 31 & 210733 & Pennsylvania & --- & 3.8 & 2.1 & 22.5 & 36.3 \\
\hline 32 & 163921 & Florida & -- & 3.5 & 1.5 & --- & --- \\
\hline
\end{tabular}


Table 5 - Financial and operating statistics of class I, and class II auto carriers (2002)

\begin{tabular}{|c|c|c|c|c|c|c|c|}
\hline$\#$ & $\begin{array}{c}\text { Motor } \\
\text { Carrier } \\
\#\end{array}$ & State & $\begin{array}{c}\text { Total } \\
\text { Operational } \\
\text { Revenue } \\
\text { (\$ million) } \\
\end{array}$ & $\begin{array}{c}\text { Total } \\
\text { Operational } \\
\text { Expenses } \\
\text { (\$ million) } \\
\end{array}$ & $\begin{array}{c}\text { Miles } \\
\text { (million) }\end{array}$ & $\begin{array}{c}\text { Tons } \\
\text { (thousand) }\end{array}$ & $\begin{array}{c}\text { Ton- } \\
\text { Miles } \\
\text { (million) }\end{array}$ \\
\hline 1 & 42537 & Illinois & 176.2 & 174.2 & 64.4 & 3487.5 & 867.2 \\
\hline 2 & 71902 & Missouri & 120.5 & 130.2 & 45.7 & 2440.0 & 697.0 \\
\hline 3 & 112391 & Michigan & 103.3 & 95.8 & 29.8 & 3338.7 & 294.6 \\
\hline 4 & 177129 & Colorado & 88.8 & 88.3 & 34.2 & 947.4 & 439.6 \\
\hline 5 & 215978 & Missouri & 64.8 & 60.7 & 16.6 & 1624.6 & 272.2 \\
\hline 6 & 249800 & Texas & --- & 59.6 & 19.9 & 137.9 & 47.3 \\
\hline 7 & 134614 & Washington & 24.5 & 27.6 & 12.0 & 281.2 & --- \\
\hline 8 & 226461 & Texas & 24.4 & 17.7 & 8.4 & --- & 123.8 \\
\hline 9 & 133993 & Alabama & --- & 14.0 & --- & --- & --- \\
\hline 10 & 36824 & Texas & --- & 12.8 & 6.4 & --- & --- \\
\hline 11 & 363019 & Florida & --- & 11.8 & --- & --- & --- \\
\hline 12 & 210561 & Arizona & 12.4 & 11.8 & 3.0 & --- & --- \\
\hline 13 & 148860 & Maryland & --- & 10.5 & 4.1 & 171.3 & --- \\
\hline 14 & 255969 & Michigan & 7.3 & 9.5 & 4.5 & 143.7 & 40.9 \\
\hline 15 & 153385 & Colorado & --- & 9.4 & 3.8 & --- & 52.2 \\
\hline 16 & 117380 & Nevada & --- & 8.8 & 1.2 & --- & --- \\
\hline 17 & 98938 & Massachusetts & --- & 8.8 & 2.6 & 54.0 & --- \\
\hline 18 & 174971 & California & --- & 7.9 & 2.4 & --- & 39.8 \\
\hline 19 & 106205 & New York & --- & 7.8 & 1.3 & --- & --- \\
\hline 20 & 174127 & Missouri & --- & 6.9 & --- & --- & --- \\
\hline 21 & 143453 & New Jersey & --- & 6.7 & 2.6 & --- & 5.1 \\
\hline 22 & 250710 & Indiana & --- & 4.8 & --- & --- & --- \\
\hline 23 & 210733 & Pennsylvania & 5.4 & 4.7 & 2.3 & 24.2 & 39.5 \\
\hline 24 & 163921 & Florida & --- & 3.7 & 1.5 & --- & --- \\
\hline
\end{tabular}


Table 6 - Financial and operating statistics of class I, and class II auto carriers (2003)

\begin{tabular}{cccccccc}
\hline \# & $\begin{array}{c}\text { Motor } \\
\text { Carrier } \\
\#\end{array}$ & State & $\begin{array}{c}\text { Total } \\
\text { Operational } \\
\text { Revenue } \\
\text { (\$ million) }\end{array}$ & $\begin{array}{c}\text { Total } \\
\text { Operational } \\
\text { Expenses } \\
\text { (\$ million) }\end{array}$ & $\begin{array}{c}\text { Miles } \\
\text { (million) }\end{array}$ & $\begin{array}{c}\text { Tons } \\
\text { (thousand) }\end{array}$ & $\begin{array}{c}\text { Ton- } \\
\text { Miles } \\
\text { (million) }\end{array}$ \\
\hline 1 & 26396 & Montana & --- & 179.4 & 89.4 & --- & --- \\
2 & 42537 & Illinois & 174.2 & 176.8 & 59.8 & 3653.4 & 954.5 \\
3 & 71902 & Missouri & 127.3 & 137.3 & 46.8 & 2563.6 & 750.7 \\
4 & 112391 & Michigan & --- & 96.3 & 28.0 & 3176.2 & --- \\
5 & 177129 & Colorado & 91.1 & 90.8 & 35.0 & 1044.9 & --- \\
6 & 249800 & Texas & 71.3 & 70.8 & 21.4 & 144.4 & 45.9 \\
7 & 215978 & Missouri & 6.6 & 64.7 & 16.9 & 1691.5 & 269.7 \\
8 & 134614 & Washington & 29.6 & 34.9 & 14.0 & 372.0 & --- \\
9 & 36824 & Texas & 14.8 & 14.5 & 6.9 & --- & --- \\
10 & 363019 & Florida & --- & 12.7 & 7.0 & --- & --- \\
11 & 210561 & Arizona & --- & 12.3 & 3.3 & --- & --- \\
12 & 117380 & Nevada & --- & 11.7 & 1.7 & --- & --- \\
13 & 255969 & Michigan & 8.1 & 10.8 & 5.0 & 160.8 & --- \\
14 & 153385 & Colorado & --- & 10.0 & 4.1 & --- & 66.0 \\
15 & 148860 & Maryland & 10.4 & 9.8 & 4.2 & 165.9 & --- \\
16 & 174127 & Missouri & --- & 8.0 & --- & -- & --- \\
17 & 106205 & New York & --- & 7.5 & 1.1 & --- & --- \\
18 & 250710 & Indiana & --- & 5.1 & --- & --- & --- \\
19 & 225762 & Texas & 0.6 & 4.6 & 0.2 & --- & --- \\
20 & 210733 & Pennsylvania & 4.2 & 3.8 & 1.6 & 24.9 & 27.4 \\
\hline
\end{tabular}


To haul the cars over shorter distances, there are smaller 2-3 car capacity trailers (called "Hotshot") that are pulled by a pick-up or small trucks. Hotshots are also used for classics or other valuable vehicles. Other smaller carriers are "Flat-Bed" or "Drop-Deck" Trailers, which are used to haul larger Trucks or vans.

In some cases, it is necessary to unload some of the cars in order to make a delivery. This would be a crucial factor that increases the risk of damages to the cars depending on drivers' training and experience. Customers can minimize this risk by requesting a particular spot on the trailer for their car. The position of a car on the trailer is determined based on the size and weight of the vehicle. Safety regulations on the height, the length and the weight play a major role in loading cars on a multi-car trailer.

Some auto transport companies offer special services, i.e. express delivery, door-to-door, or enclosed transport with some additional charges. Among those services, the so-called door-to-door service is a misconception, since the size of most trailers makes it extremely difficult to maneuver them through residential neighborhoods. There is also a possibility of damages from tree branches to the cars that are loaded on the top rack. So, usually the car is unloaded in a terminal close to the final destination, and either the customer picks up the car or the final delivery is done using smaller trucks.

Table 7 and Figure 22 show some sample quote prices for car delivery from College Park, Maryland to different destinations within the United States. As shown in the diagram, the rates increase linearly up to the point where the delivery distance is roughly equal to 1000 miles. From that point forward there a smaller change in rate with respect to the increase in delivery distance. Usually, different rates are offered by different carriers. Their rates also change differently when the delivery distance increases. This is mainly related to the location of the satellite and breakbulk terminals, the fleet size and the efficiency of the trucking operations.

As discussed earlier customers can check the status of the progress of shipment and track them either online or by calling the carrier but they don't guarantee a delivery. Customers can check the status of the progress of shipment and track them either online or by calling the carrier but delivery date is not guaranteed. 
Table 7 - Auto transport cost for different origin/destination (online quote)

\begin{tabular}{cccccc}
\hline$\#$ & Origin & Destination & $\begin{array}{c}\text { Distance } \\
(\mathbf{m i l e s )}\end{array}$ & $\begin{array}{c}\text { Standard Transport } \\
\text { Cost (\$) }\end{array}$ & $\begin{array}{c}\text { Enclosed Transport } \\
\text { Cost (\$) }\end{array}$ \\
\hline 1 & College Park, MD & Richmond, VA & 120 & 475 & 730 \\
2 & College Park, MD & Albany, NY & 380 & 575 & 890 \\
3 & College Park, MD & Columbus, OH & 420 & 575 & 890 \\
4 & College Park, MD & Boston, MA & 430 & 575 & 890 \\
5 & College Park, MD & Chicago, IL & 700 & 660 & 1030 \\
6 & College Park, MD & Miami, FL & 1060 & 890 & 1390 \\
7 & College Park, MD & Dallas, TX & 1340 & 875 & 1345 \\
8 & College Park, MD & Salt lake City, UT & 2080 & 890 & 1390 \\
9 & College Park, MD & San Diego, CA & 2710 & 990 & 1530 \\
10 & College Park, MD & Sacramento, CA & 2730 & 990 & 1530 \\
\hline
\end{tabular}

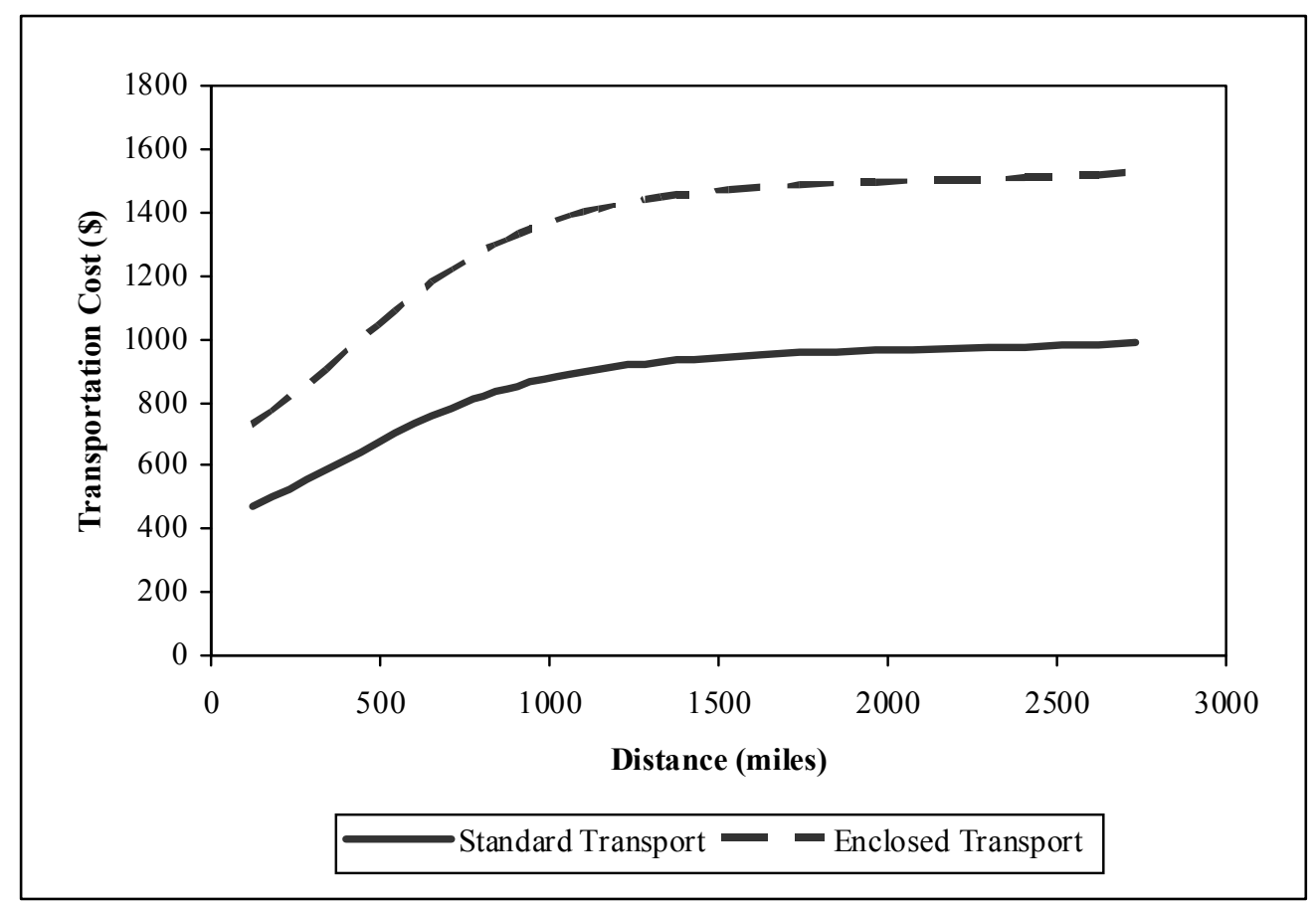

Figure 22 - Auto transport cost for different delivery distances (online quote) 
In most cases the delay is duo to the fact that they don't have enough loads to consolidate and dispatch to the next hub on their way to their destination, so loads have to wait at the terminal. There are a large number of online complaints that have been posted by unsatisfied customers mostly because of delays in delivery.

\section{Network with 5 Breakbulks}

The main goal of performing the first set of numerical experiments is to check the accuracy of the mathematical formulation, and the 3 computer programs that have been discussed earlier. As shown in Figure 23 an auto carrier company operates on a 5-terminal network and provides consolidation transportation service. It is assumed that the fleet of 15 trucks is homogeneous and each vehicle can carry up to 10 cars. The network structure, empty truck locations and shipment information are shown in Figure 23. 10 requests for shipments (total of 200 cars) arrive at time 0 . The number of time periods in the planning horizon is assumed to be equal to 10 .

The mathematical formulation is generated using the "Problem Generator". It contains 1910 variables and 324 constraints. The problem is solved in 58 sec. using CPLEX on a Pentium M (1.60GHz) machine. Based on the optimal solution the "Operation Plan" program generates the performance measures and the details of shipments/trucks routing plan. The performance measures are presented in Figure 24. 197 cars are delivered within the planning horizon. The average fill rate of the trucks is $97.9 \%$, and the total cost is equal to 142.55 units. Based on the recommended plan, there will be 29 trips by loaded trucks along with 3 additional empty trips.

To check the accuracy of mathematical formulation and the computer programs the time-space diagram of the operation is designed in AutoCAD based on the routing and dispatching plans. Figure 25 shows routing of shipments 1, 2, 3, 4, and 7 and Figure 26 illustrates the dispatching plan for all trucks. The number of trucks on each link is shown in a small box on the link. These diagrams were used to verify the accuracy of the components of the mathematical formulation. It was also used during the process of debugging the computer programs that were developed in this research. 
- LTL Network structure:

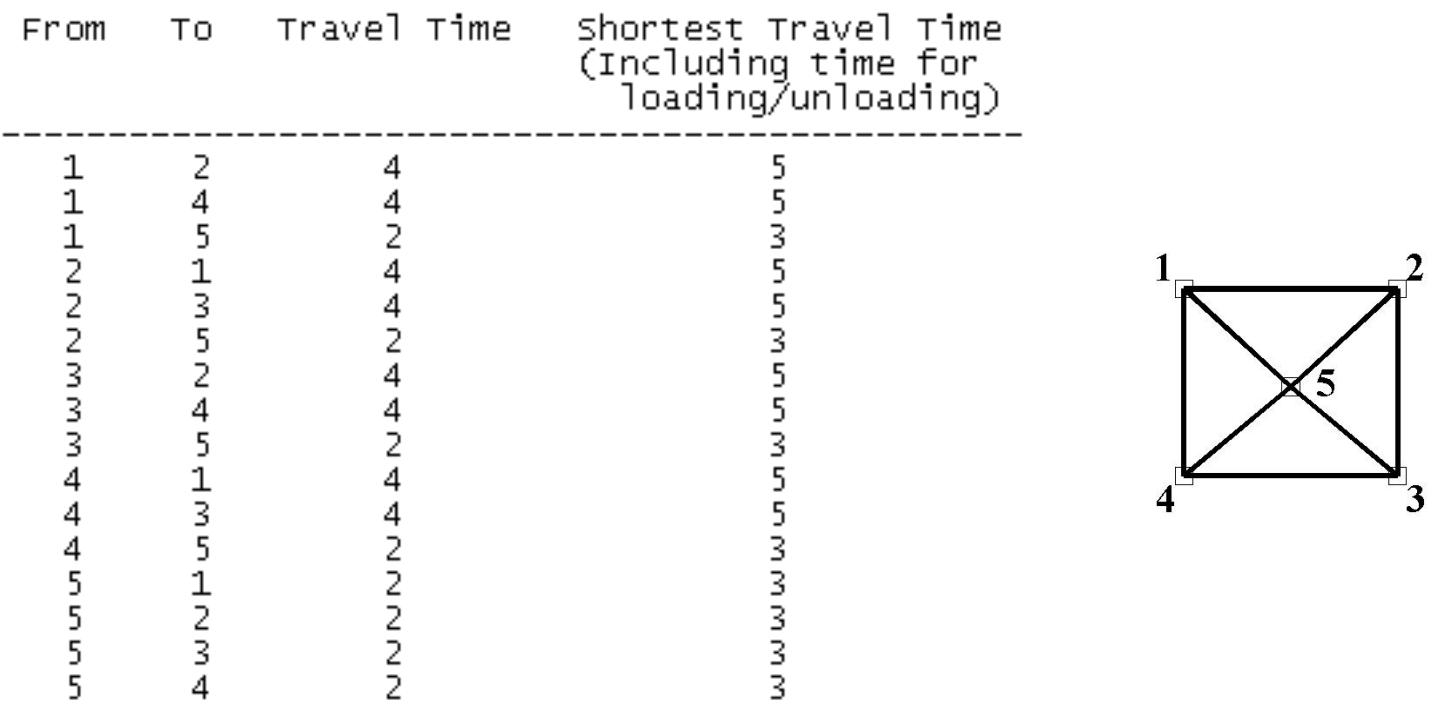

- Empty Truck Locations:

$\begin{array}{ccc}\text { Termina } 1 & \text { Time } & \text { Empty Truck } \\ -1 & 0 & 3 \\ 2 & 0 & 3 \\ 3 & 0 & 3 \\ 4 & 0 & 3 \\ 5 & 0 & 3\end{array}$

- LTL Shipments:

\begin{tabular}{|c|c|c|c|c|c|c|c|c|c|c|}
\hline shipment & SN & Size & origin & Destin & ET & LT & $\mathrm{CH}$ & $C w^{\prime}$ & $C N$ & $\mathrm{CL}$ \\
\hline 1 & 1 & 24 & 1 & 2 & 0 & 5 & 0.05 & 0.05 & 0.30 & 0.05 \\
\hline$\overline{2}$ & $\overline{2}$ & 28 & 1 & 3 & 0 & 5 & 0.05 & 0.05 & 0.30 & 0.05 \\
\hline 3 & 3 & 19 & $\overline{2}$ & 3 & 0 & 5 & 0.05 & 0.05 & 0.30 & 0.05 \\
\hline 4 & 4 & 23 & 2 & 4 & 0 & 5 & 0.05 & 0.05 & 0.30 & 0.05 \\
\hline 5 & 5 & 16 & 5 & 4 & 0 & 5 & 0.05 & 0.05 & 0.30 & 0.05 \\
\hline 6 & 6 & 17 & 4 & 1 & 0 & 5 & 0.05 & 0.05 & 0.30 & 0.05 \\
\hline 7 & 7 & 15 & 1 & $\overline{3}$ & 0 & 5 & 0.05 & 0.05 & 0.30 & 0.05 \\
\hline 8 & 8 & 27 & 5 & 4 & 0 & 5 & 0.05 & 0.05 & 0.30 & 0.05 \\
\hline 9 & 9 & -16 & 3 & 2 & 0 & 5 & 0.05 & 0.05 & 0.30 & 0.05 \\
\hline 10 & 10 & 15 & $\overline{3}$ & 4 & 0 & 5 & 0.05 & 0.05 & 0.30 & 0.05 \\
\hline
\end{tabular}

- Problem Formulation:

- Number of shipment variables $=1620$

- Number of Fult Truck Variables = 1-12

- Number of Empty Truck variables = 178

- Total Number variables $\quad=1910$

- Number of shipment conservation constraints $=50$

- Number of Truck Conservation Constraints $=50$

- Number of shipment-Truck Connection Constraints = 224

- Tota1 Number of Constraints $\quad=324$

Figure 23 - Problem characteristics (Numerical experiment 1 - Base case) 


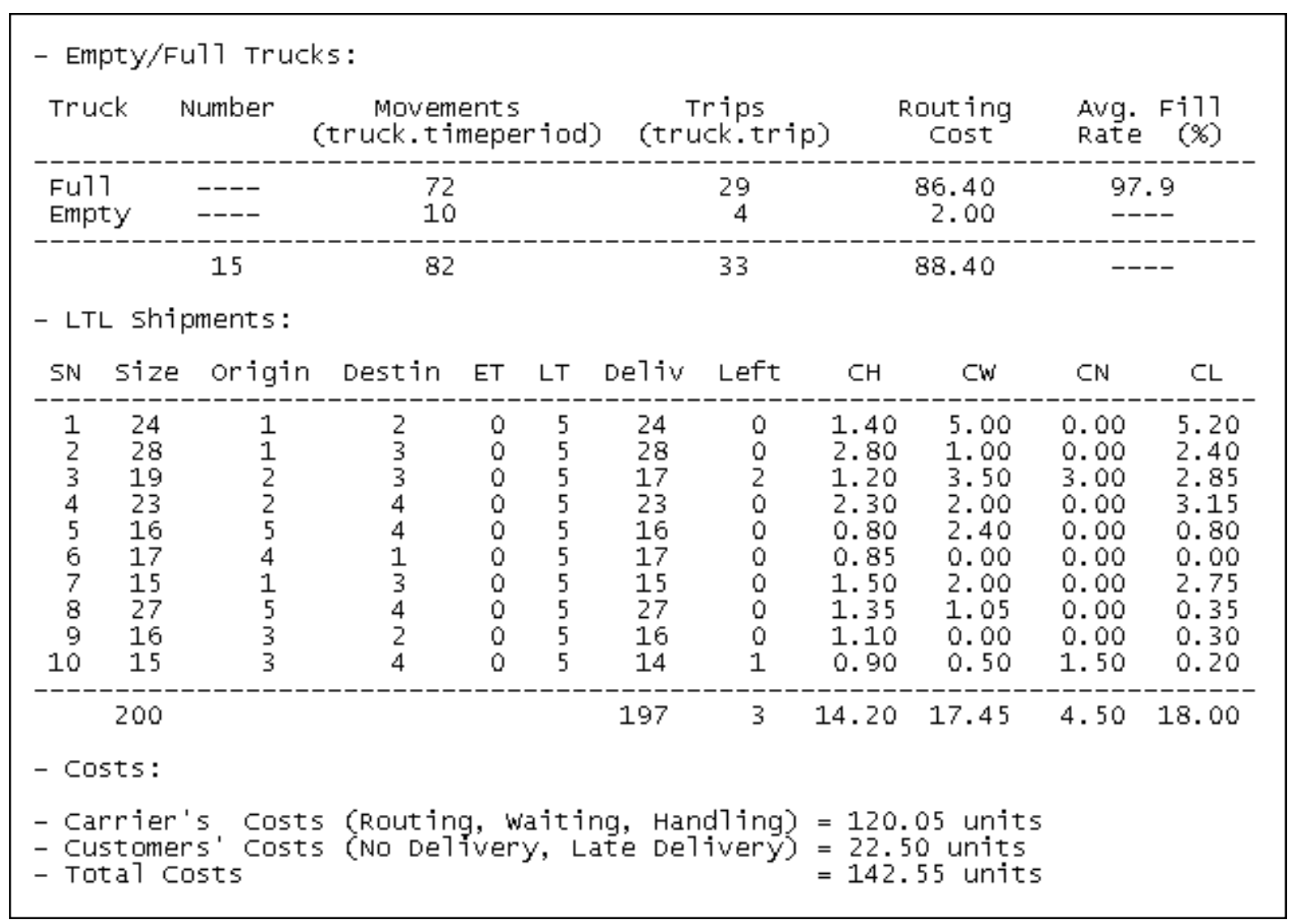

Figure 24 - Performance measures (Numerical experiment 1) 


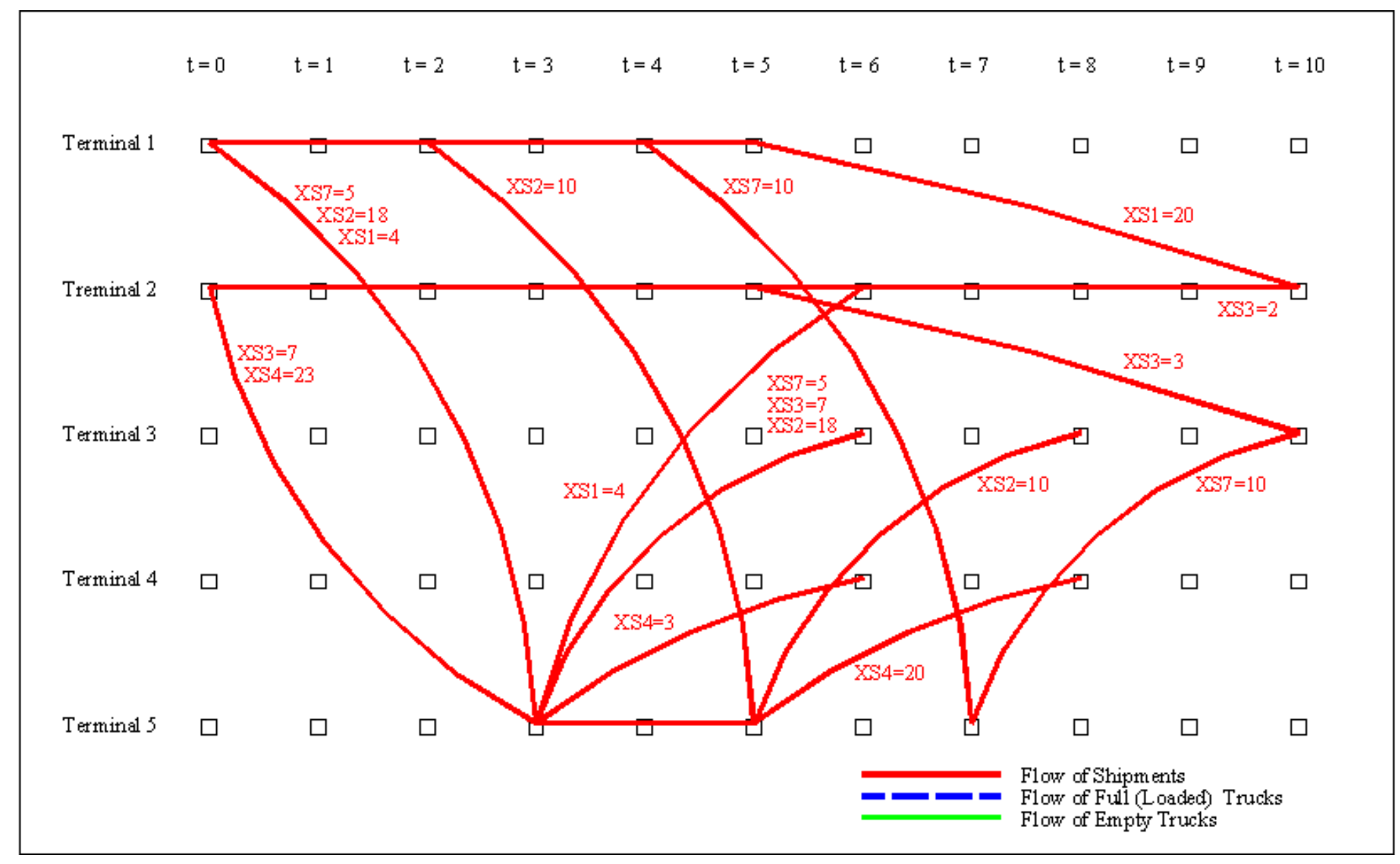

Figure 25 - Flow of shipments in optimal solution (Numerical experiment 1) 


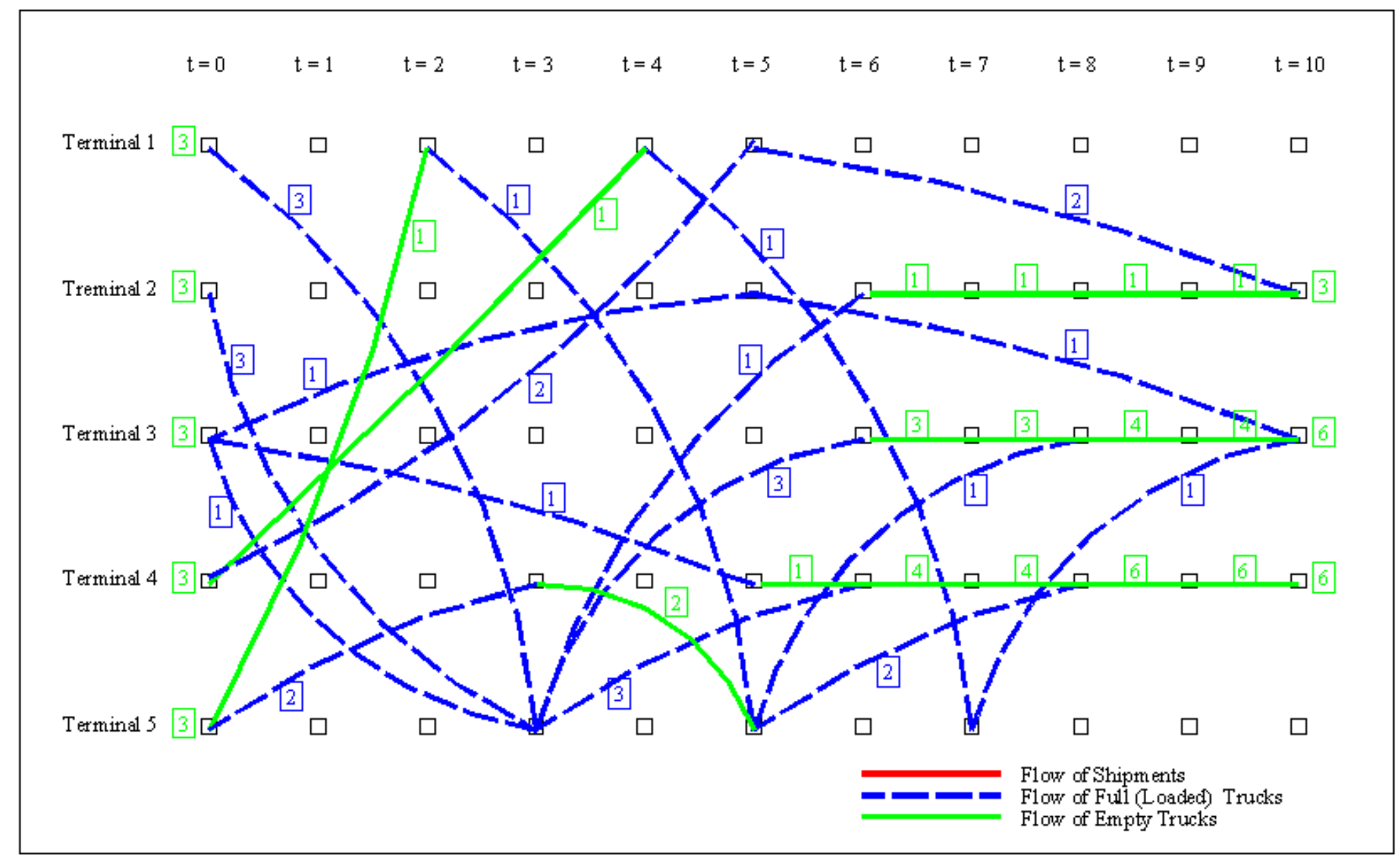

Figure 26 - Flow of full and empty trucks in optimal solution (Numerical experiment 1) 


\section{Network with 10 Breakbulks}

This section presents the second set of numerical experiments that is conducted to set the problem parameters and study the sensitivity of the solution with respect to changes in the contributing factors.

Figure 27 shows the medium-size network that is used in this section. This is a more realistic problem compared to the first computational effort that is described in the previous section. The network consists of 20 undirected links that connect 10 breakbulk terminals that are located in the proximity of 10 major cities in the US. All links are assumed to be approximately 600 miles long. A fleet of auto-carrier trucks is providing auto transport service by covering a region larger than half of the United States. If total of 40 shipments arrive per day, considering the average of $\$ 600$ revenue/shipment the trucking company is a Class II motor carrier with over $\$ 8 \mathrm{M} /$ year revenue (referring to the online quotes listed in Table 7). A set of preliminary numerical experiments have been conducted considering different fleet sizes and number of time periods to set the base values for the problem parameters. For the base case, the demand is fully satisfied when the number of time periods is 9 and the fleet size is 10. The problem characteristics are shown in Figure 28. The problem is solved using CPLEX. Figure 29 shows the performance measures that are generated using “Operation Plan” program. 


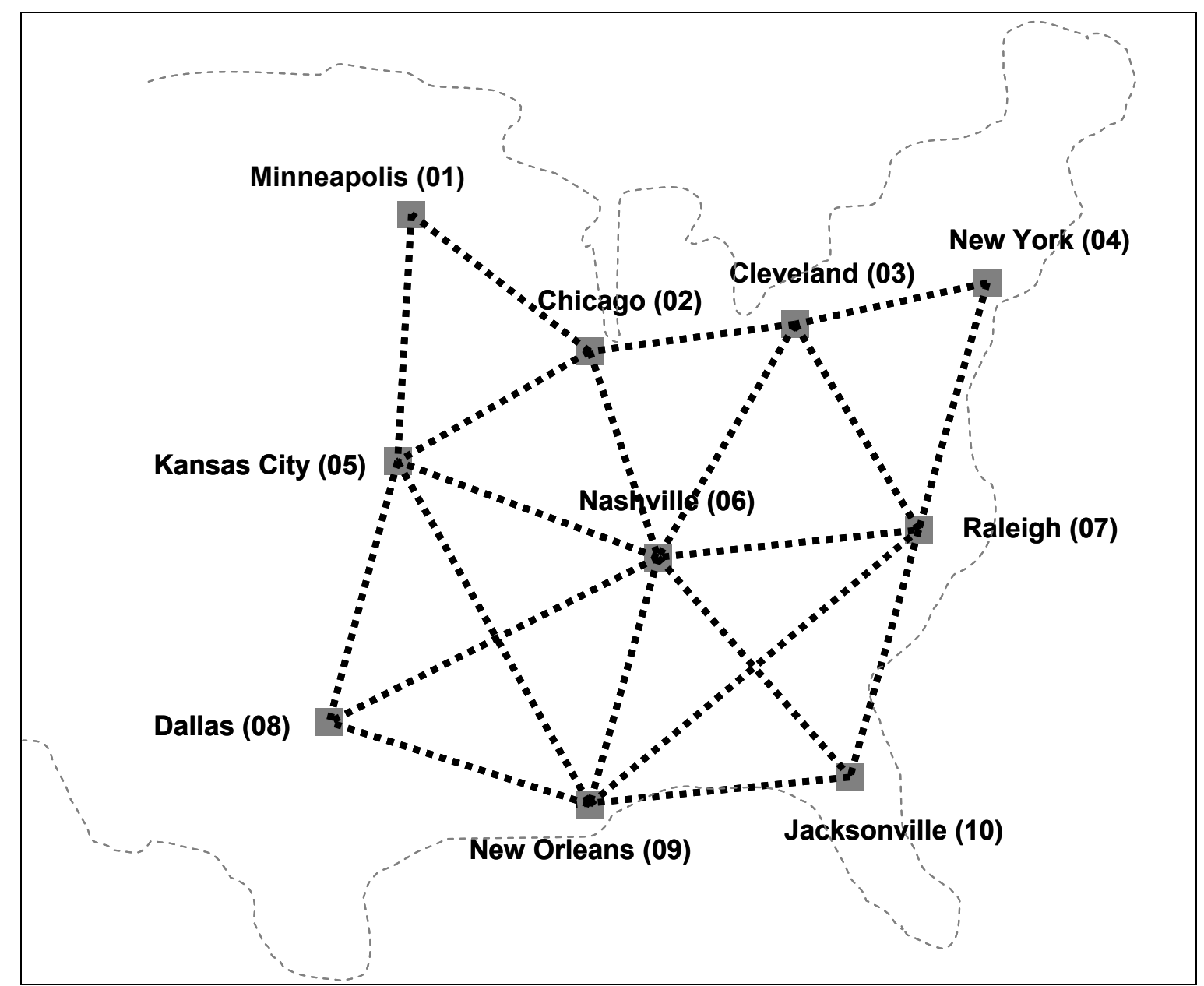

Figure 27- Physical network (10-terminal) 


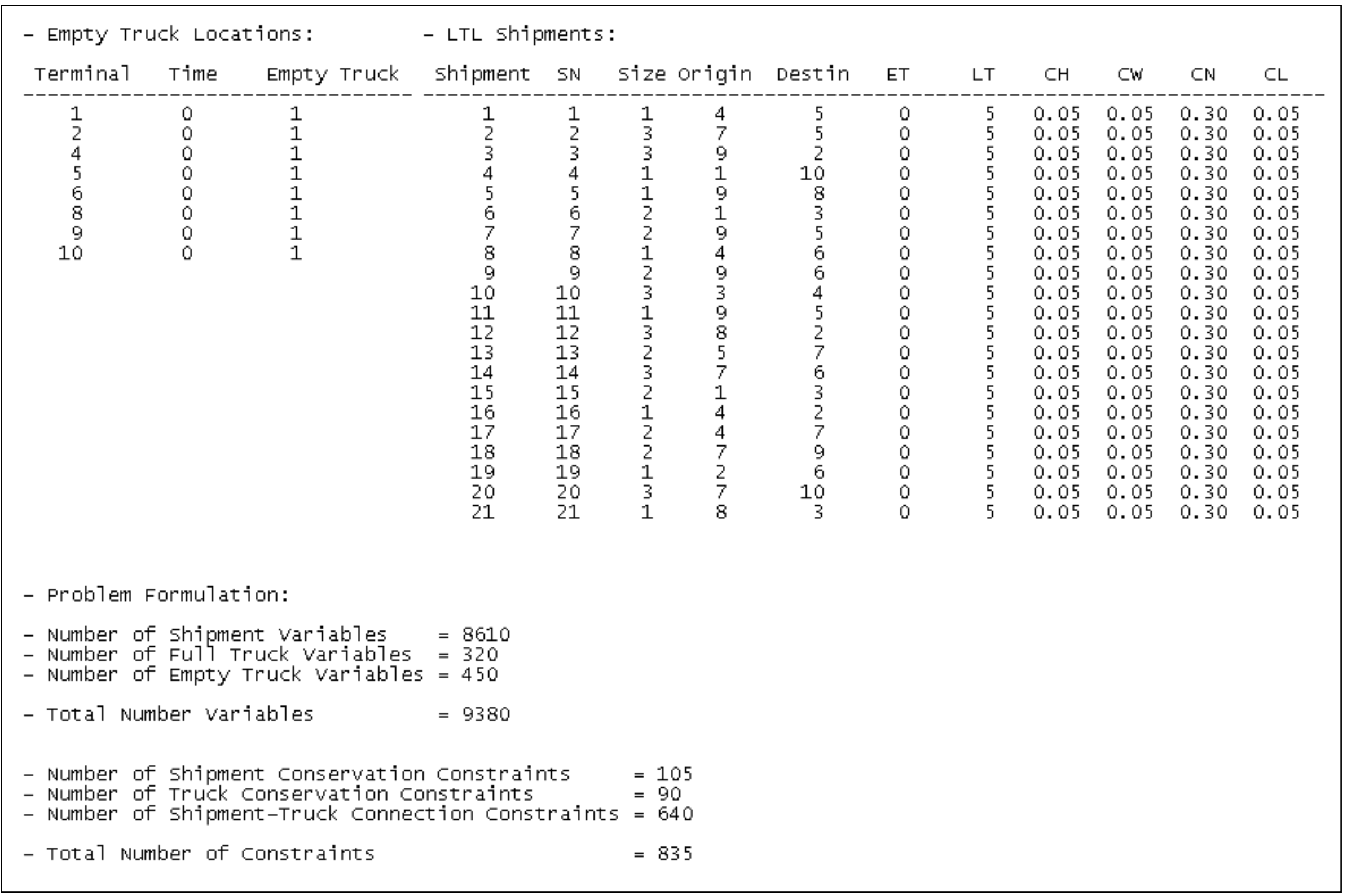

Figure 28 - Problem characteristics (Numerical experiment 2 - Base case) 


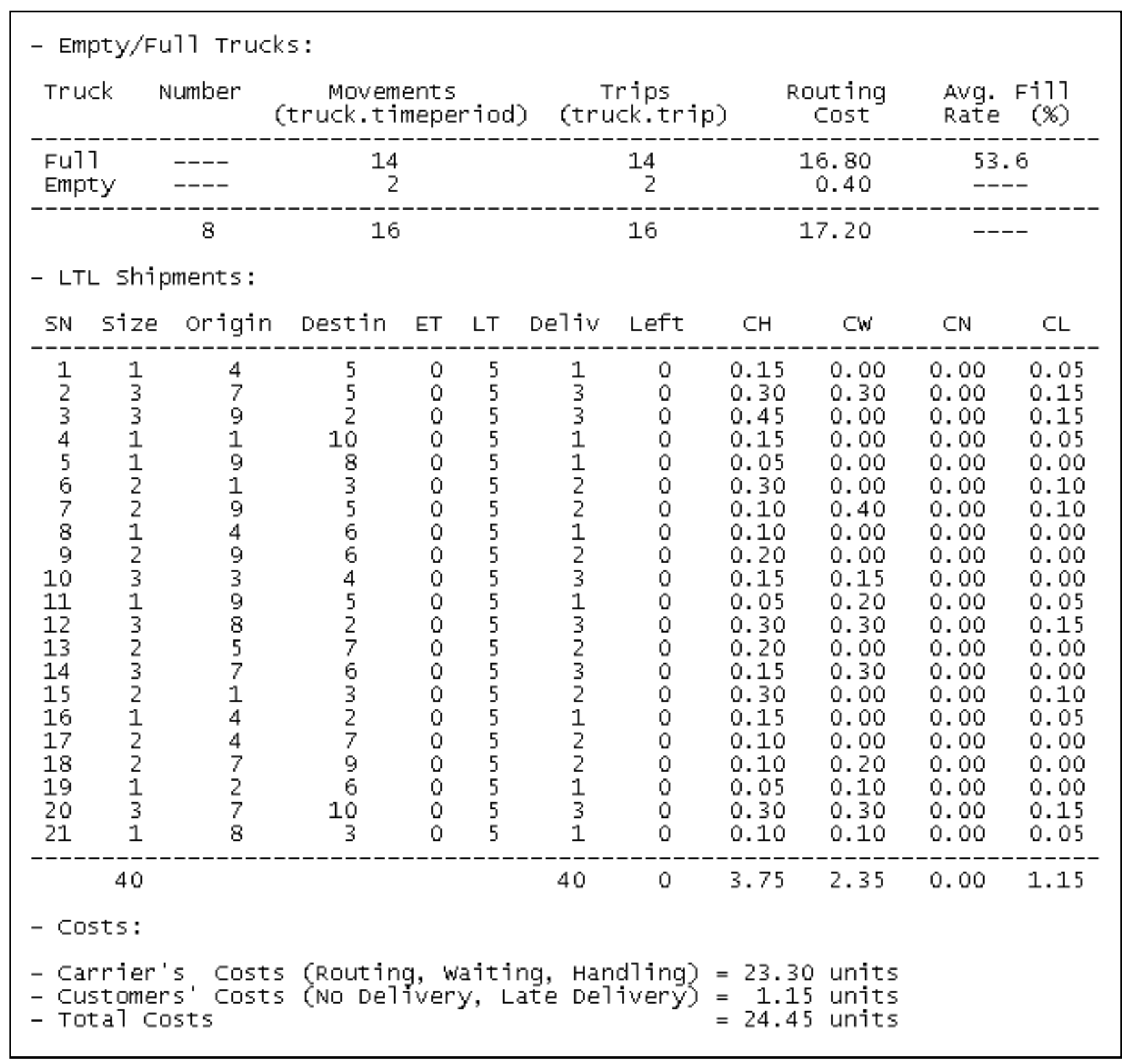

Figure 29 - Performance measures for the optimal solution (Numerical experiment 2 - Base case) 
The shipment routing and truck scheduling plan that is generated using the optimization method depends on the following parameters:

- Length of planning horizon

- Demand characteristics

○ Shipment size

- Shipment origin/destination

- Shipment handling cost factors

○ Shipment waiting cost factor

- Shipment delivery failure cost factor

- Shipment late delivery cost factor

○ Shipment early delivery cost factor

- Supply characteristics

- Number of service links

○ Fleet size

○ Empty truck locations

In this section, by changing the above parameters of the problem, 10 different cases are created. The first case is a parameter setting exercise, based on which the number of time periods are selected for the base case. The rest of the cases are used to analyze the system behavior. For each case all of the contributing factors are set to a constant value except one. By changing the value of the selected factor, several instances of the mathematical formulation is generated using the "Problem Generator" program and the problem is solved using CPLEX on a Pentium M $(1.60 \mathrm{GHz})$ machine. Based on the optimal solution and by running the "Operation Plan" program the performance measures are calculated and used as the basis for the analysis. The following sections present the characteristics of the cases that have been studied with detail discussion of the analysis' results. 
- Parameter Setting Experiment

Number of Time Periods

The length of the dynamic planning horizon is one of the characteristics of the time-space network that has a huge impact on the problem size. The number of time periods are determined based on the supply/demand characteristics. The goal would be to pick the number of time periods in a way that most of the demand that is generated at time zero can be delivered within the planning horizon. In this sensitivity analysis the number of time periods changed from 6 to 12 and the relative impacts are studied. The results are presented in Table 8 and Figures 30 to 33 .

As shown in Figures 30, when the number of time periods increases the customers' associated cost decreases. Assuming that no other request is received during the planning horizon, 9 time periods are enough for delivery of 40 shipments and further increase in the length of the planning horizon doesn't improve the solution.

As presented in Table 8 and Figure 31, by increasing the number of time periods the size of the problem (number of variables and constraints) grows linearly. However, the execution time increases exponentially. Using a lengthy planning horizon might not have a huge impact on the quality of the solution but it will definitely decrease the performance of the proposed dynamic decision making procedure.

Figure 32 and 33 illustrate the impacts of the length of the planning horizon on other performance measures. The number of deliveries increases to the point at which the demand is fully served. The average fill rate also declines due to the shipment splits and from some point onwards there is no change.

Based on the results obtained by analyzing the effects of variable number of time periods it appears that choosing the planning horizon to be equal to 9 or 10 time periods is an appropriate selection for the 10-terminal network considering the assumed supply/demand combination. 
Table 8 - Variable Number of Time Periods

\begin{tabular}{|c|c|c|c|c|c|c|c|c|c|c|c|}
\hline $\begin{array}{l}\text { Number of } \\
\text { Time Periods }\end{array}$ & $\begin{array}{l}\text { Number of } \\
\text { Variables }\end{array}$ & $\begin{array}{l}\text { Number of } \\
\text { Constraints }\end{array}$ & $\begin{array}{l}\text { Execution } \\
\text { Time (sec) }\end{array}$ & $\begin{array}{c}\text { Carrier } \\
\text { Cost (unit) }\end{array}$ & $\begin{array}{l}\text { Customer } \\
\text { Cost (unit) }\end{array}$ & $\begin{array}{c}\text { Total } \\
\text { Cost (unit) }\end{array}$ & $\begin{array}{c}\text { Loaded } \\
\text { Truck } \\
\text { Number } \\
\text { of Trips } \\
\end{array}$ & $\begin{array}{c}\text { Empty } \\
\text { Truck } \\
\text { Number } \\
\text { of Trips } \\
\end{array}$ & $\begin{array}{c}\text { Shipment } \\
\text { Delivered } \\
\text { (size) }\end{array}$ & $\begin{array}{c}\text { Shipment } \\
\text { Left } \\
\text { (size) }\end{array}$ & $\begin{array}{c}\text { Average } \\
\text { Fill Rate } \\
(\%)\end{array}$ \\
\hline 6 & 5960 & 565 & 7 & 20.35 & 3.65 & 24.00 & 12 & 2 & 35 & 5 & 60.0 \\
\hline 7 & 7100 & 655 & 22 & 21.05 & 3.20 & 24.25 & 12 & 2 & 36 & 4 & 61.7 \\
\hline 8 & 8240 & 745 & 31 & 22.25 & 2.10 & 24.35 & 13 & 2 & 38 & 2 & 56.9 \\
\hline 9 & 9380 & 835 & 128 & 23.30 & 1.15 & 24.45 & 14 & 2 & 40 & 0 & 53.6 \\
\hline 10 & 10520 & 925 & 128 & 23.30 & 1.15 & 24.45 & 14 & 2 & 40 & 0 & 53.6 \\
\hline 11 & 11660 & 1015 & 323 & 23.30 & 1.15 & 24.45 & 14 & 2 & 40 & 0 & 53.6 \\
\hline 12 & 12800 & 1105 & 269 & 23.30 & 1.15 & 24.45 & 14 & 2 & 40 & 0 & 53.6 \\
\hline
\end{tabular}

Note: Number of Links $=20$, Number of Trucks $=8$, Total Shipment Size $=40, \mathrm{WE}=1$ 


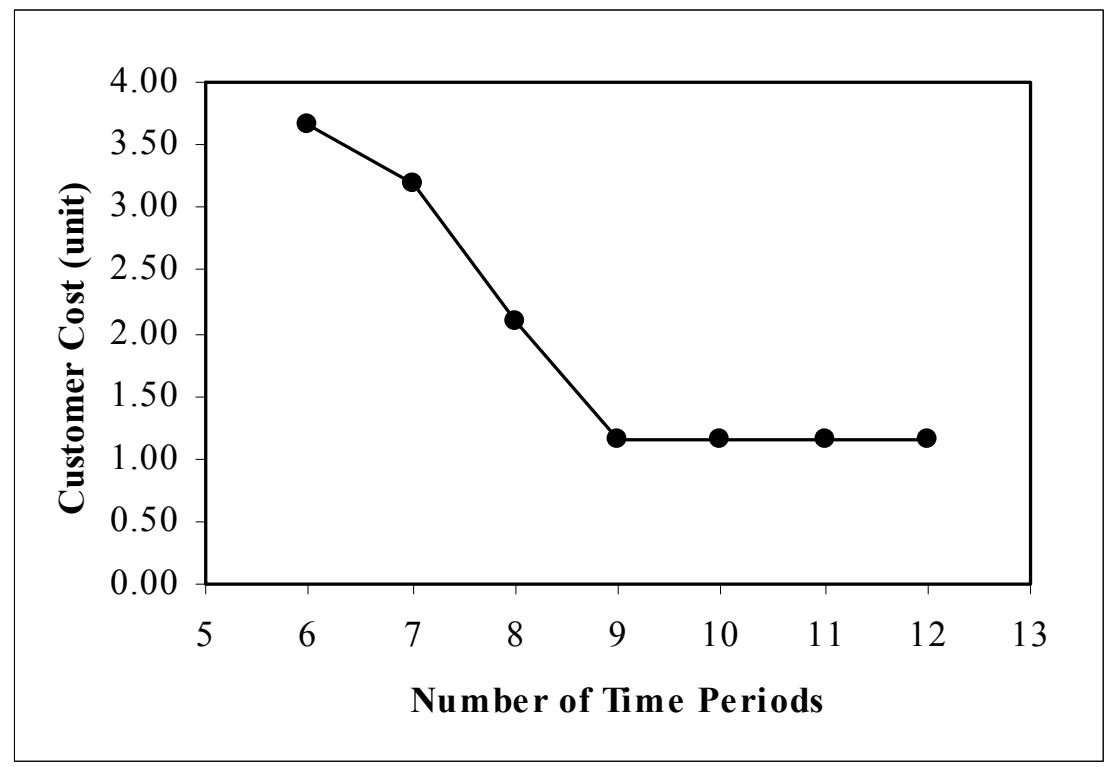

Figure 30 - Customer cost vs. Number of time periods

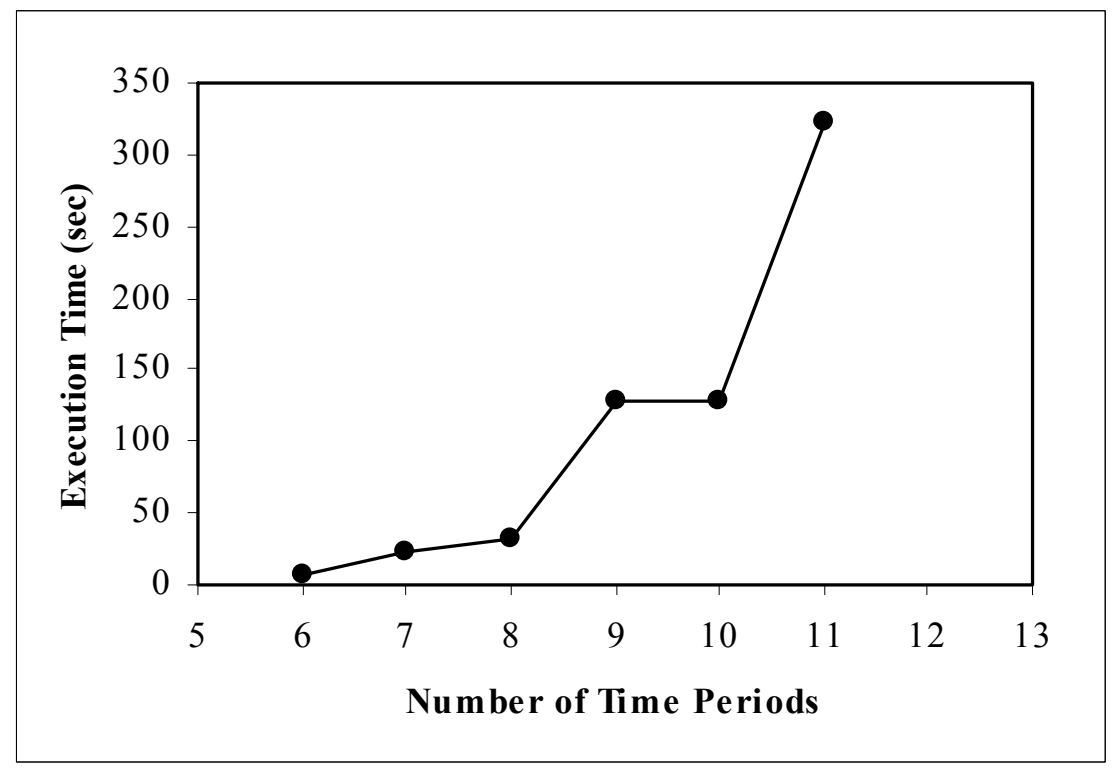

Figure 31 - Execution time vs. Number of time periods 


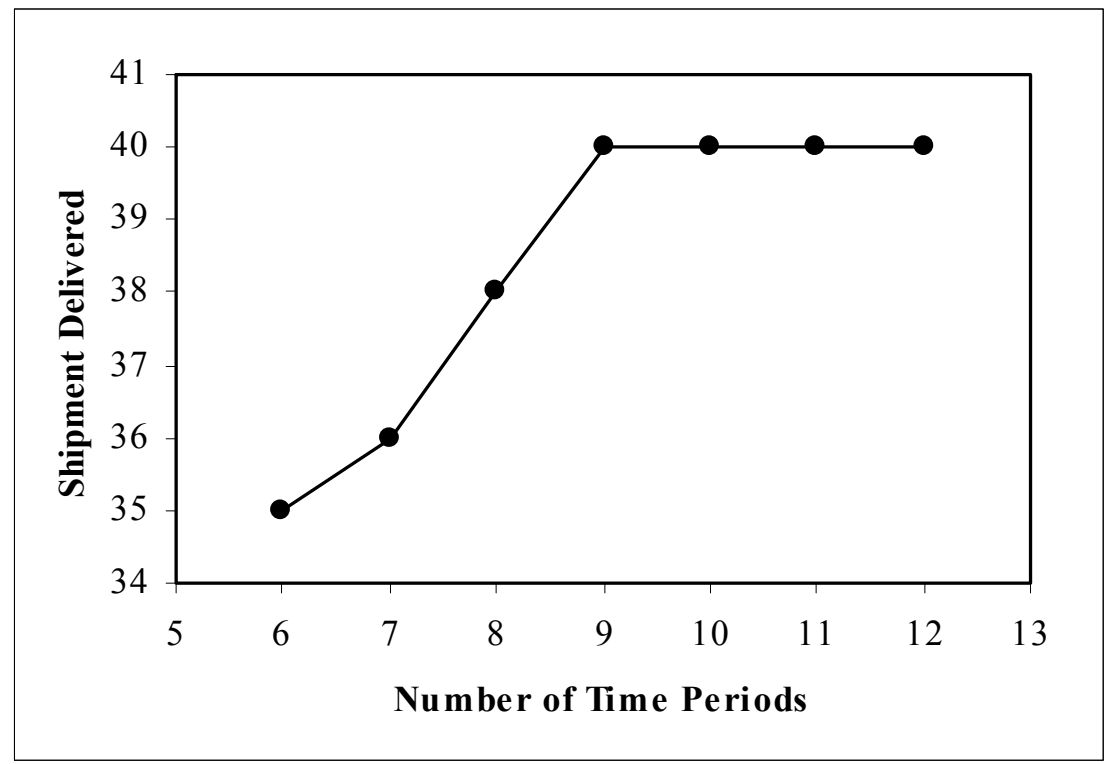

Figure 32 - Shipment delivered vs. Number of time periods

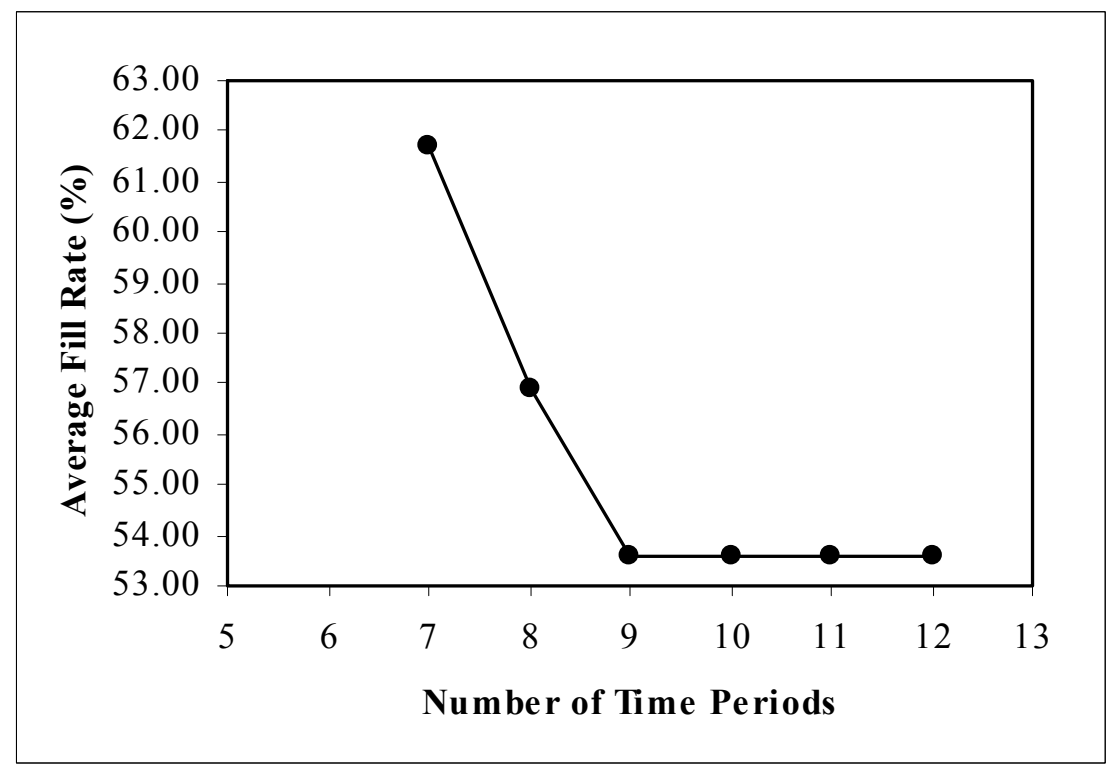

Figure 33 - Average fill rate vs. Number of time periods 
- Sensitivity Analyses

\section{Case 1: Variable Number of Trucks}

The number of trucks is among the variables that represent the supply. To analyze the sensitivity of the results with respect to changes in fleet size the problem is solved considering 7 different values for the number of trucks. Other parameters including the number of shipments, the size of shipments, the number of links and the number of time periods remain constant. Table 9 and Figures 34 to 37 show the results.

The results confirm the expectation that both the customers' associated cost and total cost decrease when more trucks are operating. However, the rate of cost reduction is declining and adding more than 10 trucks to the system doesn't improve the performance. The carrier's associated cost is increasing first due to the increase in loaded/empty truck movements. Then, it decreases when more trucks are added to the system and some of the additional trucks are not operating. Note that in this study it is assumed that all trucks are owned by the company and there is no cost associated with having a truck idle at a terminal. Considering the realistic case where a portion of fleet is leased, the total and the carrier associated cost would form "U" shape functions.

Figure 35 indicates that the average fill rate also decreases by increasing the number of trucks. When fleet size increases the demand is distributed among the available trucks, therefore there are fewer loads on each truck. Furthermore, based on Table 9 and Figures 36 and 37, there are less empty movements and more shipments are being delivered to their final destination. Overall, the results obtained from the sensitivity analysis perfectly meet the expectations regarding the impacts of the changes in fleet size.

Note that, for each sensitivity analysis, different instances of the problem are generated by changing the value of only one parameter at a time. The location of the

empty trucks at the beginning of the operation has a significant impact on the operation plan. For this case study, it is assumed that one truck is available at each 
terminal. A more appropriate way of generating the problem is to have multiple instances of randomly generated truck locations for each fleet size. If 20 different truck locations are generated for each fleet size, the total number of problem that must be solved for this case would be 140, which is beyond the scope of this limited sensitivity analysis. However, the impact of the empty truck locations is studied as a separate case and the results are presented in this Chapter. 
Table 9 - Variable Number of Trucks (Case 1)

\begin{tabular}{|c|c|c|c|c|c|c|c|c|c|c|c|}
\hline $\begin{array}{l}\text { Number of } \\
\text { Trucks }\end{array}$ & $\begin{array}{l}\text { Number of } \\
\text { Variables }\end{array}$ & $\begin{array}{l}\text { Number of } \\
\text { Constraints }\end{array}$ & $\begin{array}{l}\text { Execution } \\
\text { Time (sec) }\end{array}$ & $\begin{array}{c}\text { Carrier } \\
\text { Cost (unit) }\end{array}$ & $\begin{array}{l}\text { Customer } \\
\text { Cost (unit) }\end{array}$ & $\begin{array}{c}\text { Total } \\
\text { Cost (unit) }\end{array}$ & $\begin{array}{c}\text { Loaded } \\
\text { Truck } \\
\text { Number } \\
\text { of Trips } \\
\end{array}$ & $\begin{array}{c}\text { Empty } \\
\text { Truck } \\
\text { Number } \\
\text { of Trips } \\
\end{array}$ & $\begin{array}{l}\text { Shipment } \\
\text { Delivered } \\
\text { (size) }\end{array}$ & $\begin{array}{c}\text { Shipment } \\
\text { Left } \\
\text { (size) }\end{array}$ & $\begin{array}{c}\text { Average } \\
\text { Fill Rate } \\
\text { (\%) }\end{array}$ \\
\hline 2 & 9380 & 835 & 239 & 21.45 & 13.50 & 34.95 & 8 & 2 & 23 & 17 & 71.3 \\
\hline 4 & 9380 & 835 & 1484 & 22.95 & 4.00 & 26.95 & 12 & 3 & 37 & 3 & 63.3 \\
\hline 6 & 9380 & 835 & 193 & 22.15 & 3.15 & 25.30 & 13 & 3 & 36 & 4 & 56.2 \\
\hline 8 & 9380 & 835 & 128 & 23.30 & 1.15 & 24.45 & 14 & 2 & 40 & 0 & 53.6 \\
\hline 10 & 9380 & 835 & 37 & 22.10 & 1.40 & 23.50 & 14 & 0 & 39 & 1 & 53.6 \\
\hline 12 & 9380 & 835 & 37 & 22.10 & 1.40 & 23.50 & 14 & 0 & 39 & 1 & 53.6 \\
\hline 14 & 9380 & 835 & 37 & 22.10 & 1.40 & 23.50 & 14 & 0 & 39 & 1 & 53.6 \\
\hline
\end{tabular}

Note: Number of Time Periods $=9$, Number of Links $=20$, Total Shipment Size $=40, \mathrm{WE}=1$ 


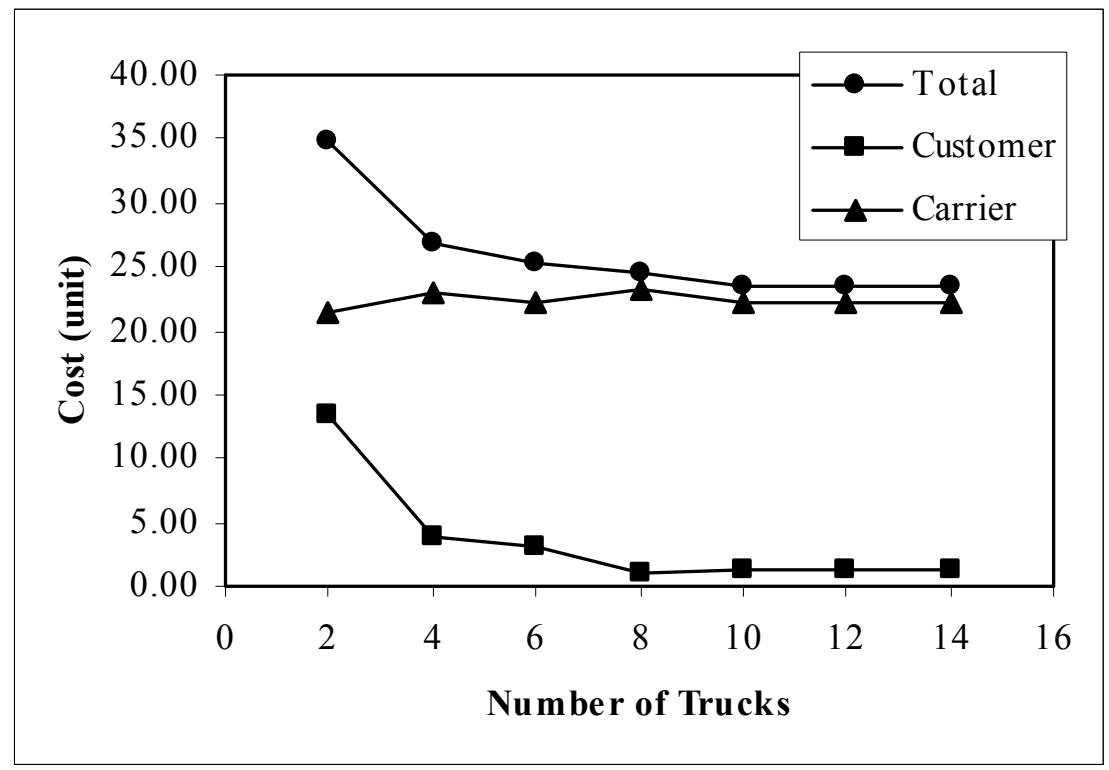

Figure 34 - Cost vs. Number of trucks (Case 1)

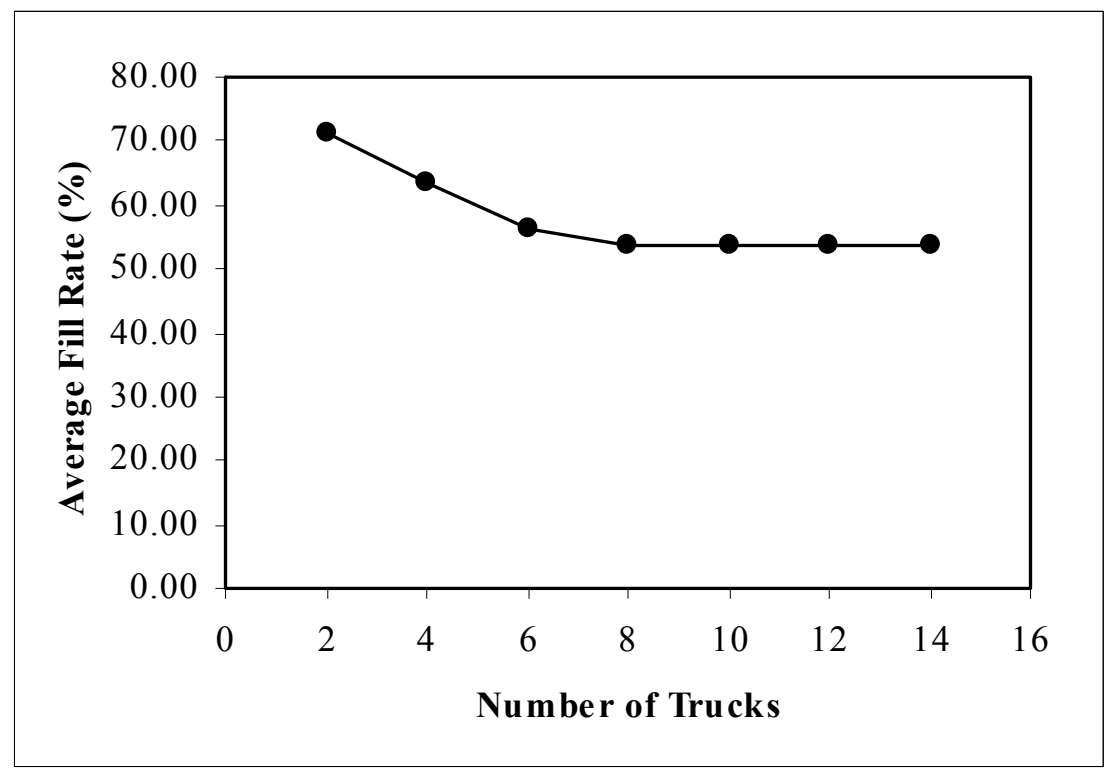

Figure 35 - Average fill rate vs. Number of trucks (Case 1) 


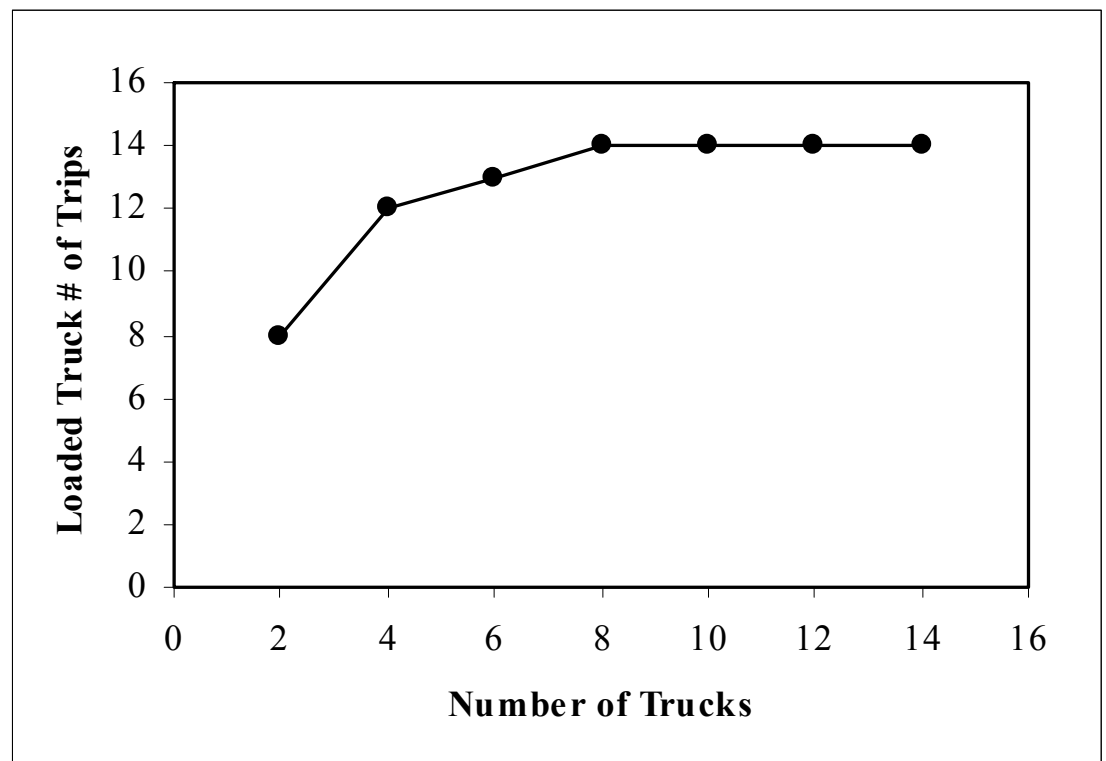

Figure 36 - Loaded truck number of trips vs. Number of trucks (Case 1)

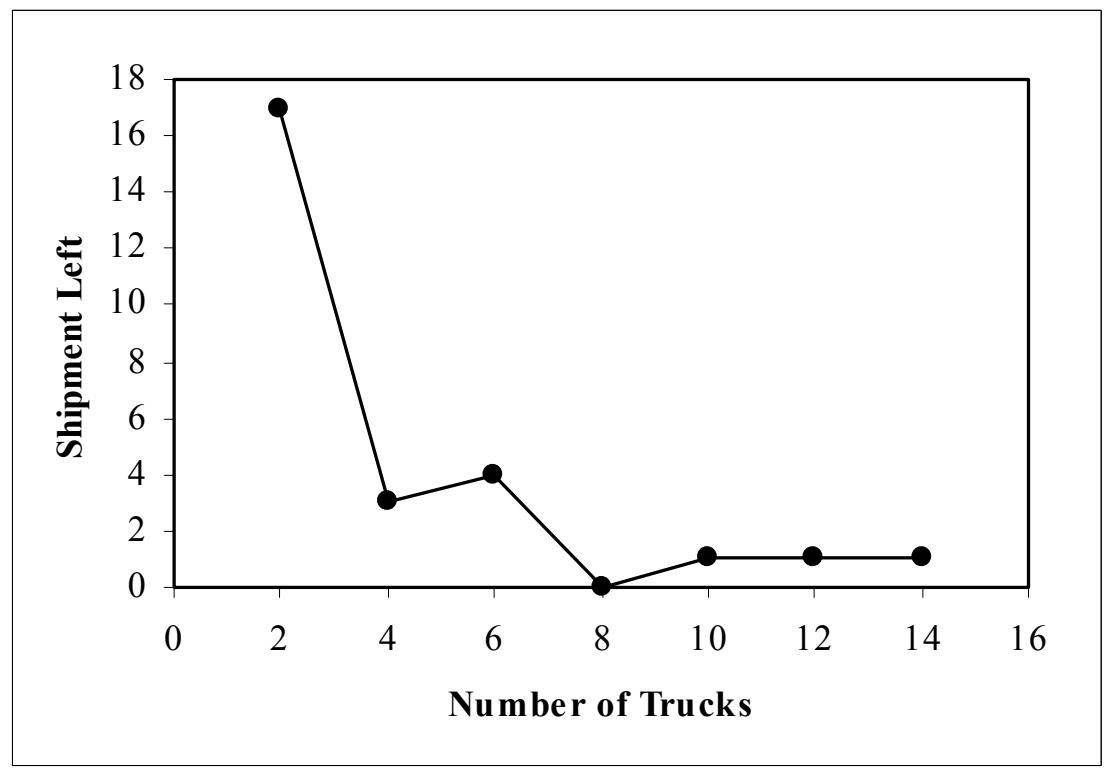

Figure 37 - Shipment left vs. Number of trucks (Case 1) 


\section{Case 2: Variable Number of Links}

As discussed earlier, it is assumed that the service network is designed through the tactical planning process. The location of terminals and the configuration of service links have been finalized prior to the operational planning. This section analyzes the impacts of the network size on the quality of trucking service and the performance of the proposed decision making procedure is analyzed in this section. The results are presented in Table 10 and Figures 38 and 39.

The original network (Figure 27) consists of 20 links. Other networks are constructed based on the original network and by removing/adding links as follow:

Network with 14 links: Remove 1-2, 2-5, 3-4, 3-7, 5-9 and 7-9

Network with 16 links: Remove 2-5, 3-7, 5-9 and 7-9

Network with 18 links: Remove 5-9 and 7-9

Network with 22 links: Add 1-9 and 4-9

Network with 24 links: Add 2-8, 3-10, 1-9 and 4-9

Network with 26 links: Add 1-6, 4-6, 2-8, 3-10, 1-9 and 4-9

It is assumed that all service links have equal length. The number of links is one of the variables that represent the level of supply. As shown in Figure 38, by increasing the number of links and expanding the network size there are better paths available to route the shipments efficiently. This means that customers experience less delay in service and as a result the customers' associated cost and therefore the total cost are decreasing.

By growing the network size and increasing the number of service links the total number of variables and constraints are increasing linearly while the execution time is increasing exponentially. As discussed earlier, one major step in all the proposed procedures is to solve the shipment and truck routing problem repeatedly. For real size networks it is not practical to use exact solution method to solve the result mathematical problem and therefore it will be necessary to develop and use the heuristic solution algorithm. 
As shown in Table 10 and Figure 39, the execution time of problem with 22 links is less than that of problem with 20 links. This is an unexpected result since the size of the problem grows by adding links to the network. However, the convergence is faster due to the configuration of the new links, the network structure and the number of branches that cancels out implicitly.

Note that, for each sensitivity analysis, different instances of the problem are generated by changing the value of only one parameter at a time. The location of the extra service links, the location of the empty trucks at the beginning of the operation and also the characteristics of demand has a significant impact on the operation plan. For this case study, a more appropriate way of generating the problem is to have multiple instances of randomly generated extra service link locations, truck locations and demand characteristics for each network size. The total number of problem that must be solved for this case is beyond the scope of this limited sensitivity analysis. However, the impact of the empty truck locations and demand characteristics are studied as separate cases and the results are presented in this Chapter. 
Table 10 - Variable Number of Links (Case 2)

\begin{tabular}{|c|c|c|c|c|c|c|c|c|c|c|c|}
\hline $\begin{array}{l}\text { Number of } \\
\text { Links }\end{array}$ & $\begin{array}{l}\text { Number of } \\
\text { Variables }\end{array}$ & $\begin{array}{l}\text { Number of } \\
\text { Constraints }\end{array}$ & $\begin{array}{l}\text { Execution } \\
\text { Time (sec) }\end{array}$ & $\begin{array}{c}\text { Carrier } \\
\text { Cost (unit) }\end{array}$ & $\begin{array}{l}\text { Customer } \\
\text { Cost (unit) }\end{array}$ & $\begin{array}{c}\text { Total } \\
\text { Cost (unit) }\end{array}$ & $\begin{array}{c}\text { Loaded } \\
\text { Truck } \\
\text { Number } \\
\text { of Trips } \\
\end{array}$ & $\begin{array}{c}\text { Empty } \\
\text { Truck } \\
\text { Number } \\
\text { of Trips } \\
\end{array}$ & $\begin{array}{c}\text { Shipment } \\
\text { Delivered } \\
\text { (size) }\end{array}$ & $\begin{array}{c}\text { Shipment } \\
\text { Left } \\
\text { (size) }\end{array}$ & $\begin{array}{c}\text { Average } \\
\text { Fill Rate } \\
(\%)\end{array}$ \\
\hline 14 & 7160 & 643 & 9 & 24.85 & 2.55 & 27.40 & 15 & 2 & 38 & 2 & 54.7 \\
\hline 16 & 7900 & 707 & 25 & 22.20 & 3.10 & 25.30 & 13 & 2 & 36 & 4 & 55.4 \\
\hline 18 & 8640 & 771 & 88 & 22.20 & 3.10 & 25.30 & 13 & 2 & 36 & 4 & 55.4 \\
\hline 20 & 9380 & 835 & 128 & 23.30 & 1.15 & 24.45 & 14 & 2 & 40 & 0 & 53.6 \\
\hline 22 & 9936 & 883 & 51 & 23.05 & 1.40 & 24.45 & 14 & 2 & 39 & 1 & 53.6 \\
\hline 24 & 10584 & 939 & 137 & 22.60 & 1.85 & 24.45 & 13 & 2 & 39 & 1 & 56.9 \\
\hline 26 & 11232 & 995 & 67 & 23.05 & 1.40 & 24.45 & 14 & 2 & 39 & 1 & 53.6 \\
\hline
\end{tabular}

Note: Number of Time Periods $=9$, Number of Trucks $=8$, Total Shipment Size $=40, \mathrm{WE}=1$ 


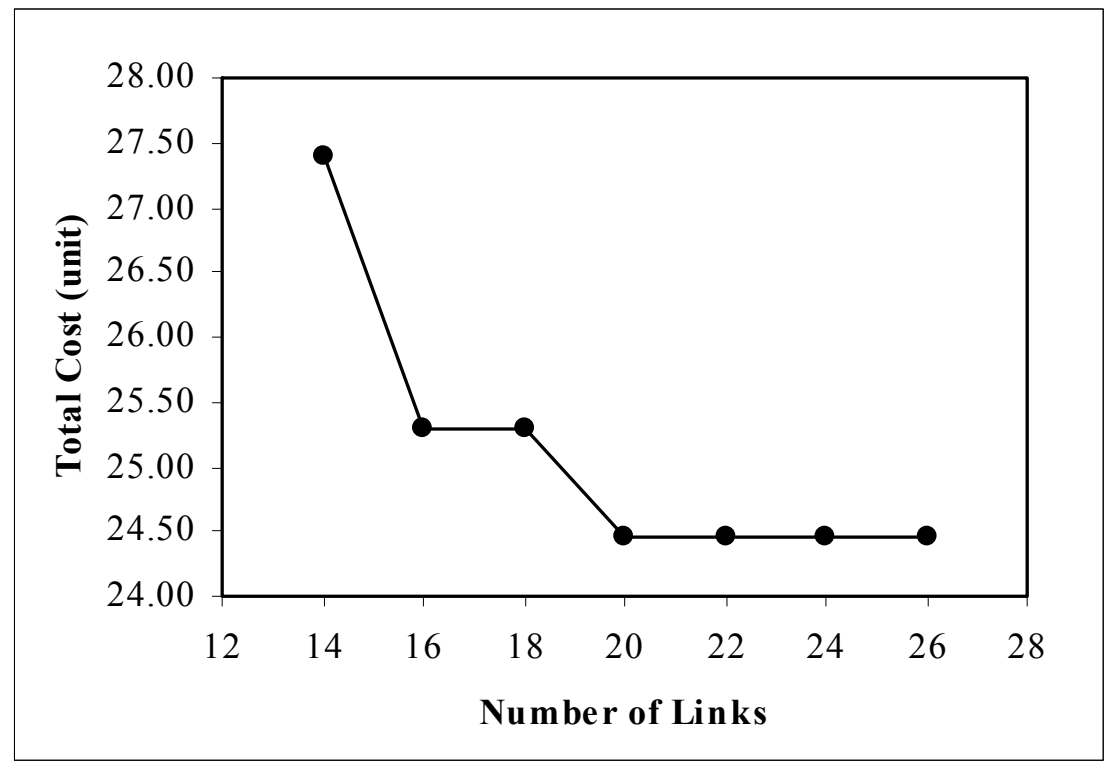

Figure 38 - Total cost vs. Number of links (Case 2)

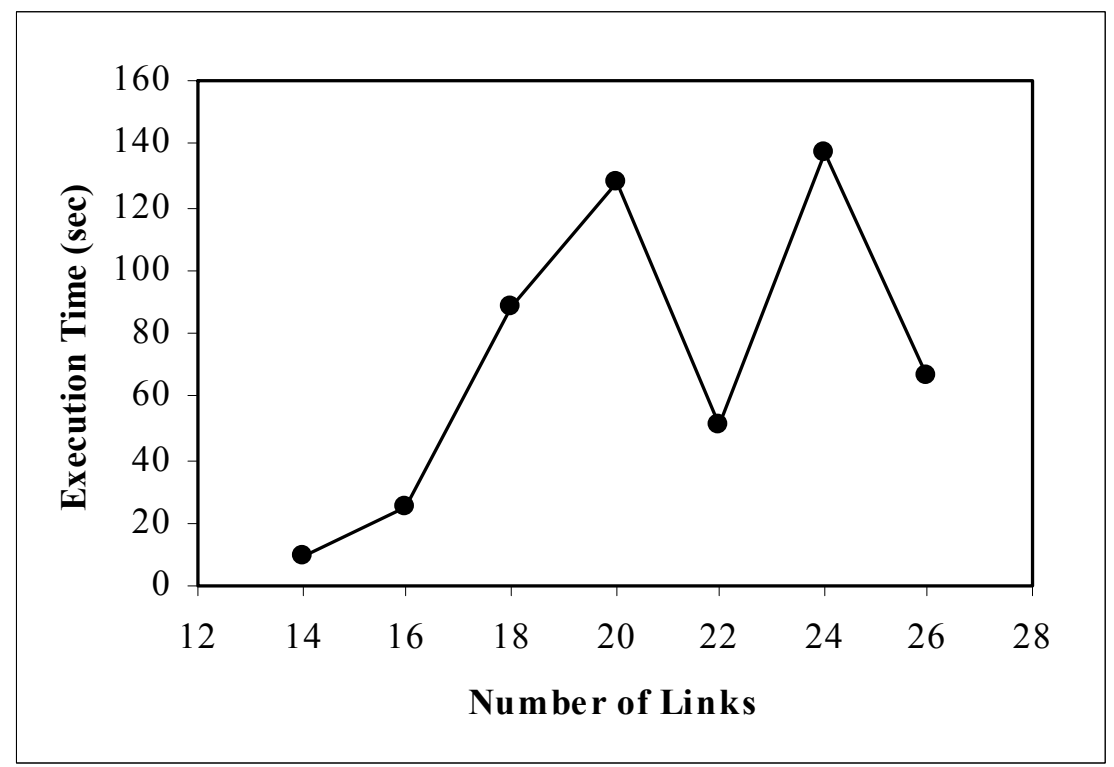

Figure 39 - Execution time vs. Number of links (Case 2) 


\section{Case 3: Variable Total Shipment Size}

This case focuses on the level of demand. Considering all other parameters are fixed, the total requested shipment size is changed from 10 cars to 70 cars per day. Table 11 and Figures 40 through 43 show the effects of shipment volume on different performance measures.

By increasing the total shipment size there will be better opportunity for shipment consolidation, therefore both the carrier's associated cost (per shipment) as well as the customers' associated cost (per shipment) are decreasing until the system reaches the saturation level. From that point onwards there is not enough supply (trucks) to provide a satisfactory level of service and the customers experience more delay.

By increasing the demand volume the number of trips (per shipment) by full trucks declines. Moreover, there will be more shipments on each truck so the average fill rate increases. All of the above observations completely match the expectations regarding the impact of increasing the demand volume on the system performance and the quality of trucking services.

Note that, for this sensitivity analysis, different instances of the problem are generated by changing the value of only one parameter, the demand volume. The characteristics of demand have a significant impact on the operation plan. For this case study, a more appropriate way of generating the problem is to have multiple instances of randomly generated demand characteristics for each demand size. This creates a large number of optimization problems that must be solved for this sensitivity analysis. As an alternative, the impact of the demand characteristics are studied as separate case and the results are presented in this Chapter. 
Table 11 - Variable Total Shipment Size (Case 3)

\begin{tabular}{|c|c|c|c|c|c|c|c|c|c|c|c|c|c|c|}
\hline $\begin{array}{c}\text { Total } \\
\text { Shipment } \\
\text { Size }\end{array}$ & $\begin{array}{l}\text { Number of } \\
\text { Variables }\end{array}$ & $\begin{array}{l}\text { Number of } \\
\text { Constraints }\end{array}$ & $\begin{array}{l}\text { Execution } \\
\text { Time (sec) }\end{array}$ & $\begin{array}{c}\text { Carrier } \\
\text { Cost (unit) }\end{array}$ & $\begin{array}{c}\text { Customer } \\
\text { Cost } \\
\text { (unit) }\end{array}$ & $\begin{array}{l}\text { Total } \\
\text { Cost } \\
\text { (unit) }\end{array}$ & $\begin{array}{c}\text { Loaded } \\
\text { Truck } \\
\text { Number } \\
\text { of Trips }\end{array}$ & $\begin{array}{c}\text { Empty } \\
\text { Truck } \\
\text { Number } \\
\text { of Trips }\end{array}$ & $\begin{array}{l}\text { Shipment } \\
\text { Delivered } \\
\text { (size) }\end{array}$ & $\begin{array}{c}\text { Shipment } \\
\text { Left } \\
\text { (size) }\end{array}$ & $\begin{array}{c}\text { Average } \\
\text { Fill Rate } \\
\quad(\%)\end{array}$ & $\begin{array}{l}\text { Customer } \\
\text { Cost } \\
\text { per } \\
\text { Shipment } \\
\text { Size }\end{array}$ & $\begin{array}{c}\text { Carrier } \\
\text { Cost } \\
\text { per } \\
\text { Shipment } \\
\text { Size } \\
\end{array}$ & $\begin{array}{c}\text { Loaded } \\
\text { Truck } \\
\text { \# of Trips } \\
\text { per } \\
\text { Shipment } \\
\text { Size } \\
\end{array}$ \\
\hline 10 & 3230 & 760 & 3 & 8.20 & 2.10 & 10.30 & 5 & 0 & 8 & 2 & 36.0 & 0.21 & 0.82 & 0.50 \\
\hline 20 & 5280 & 785 & 11 & 12.85 & 2.15 & 15.00 & 8 & 1 & 18 & 2 & 45.0 & 0.11 & 0.64 & 0.40 \\
\hline 30 & 6920 & 805 & 19 & 17.55 & 1.70 & 19.25 & 11 & 1 & 28 & 2 & 52.7 & 0.06 & 0.59 & 0.37 \\
\hline 40 & 9380 & 835 & 128 & 23.30 & 1.15 & 24.45 & 14 & 2 & 40 & 0 & 53.6 & 0.03 & 0.58 & 0.35 \\
\hline 50 & 11430 & 860 & 629 & 26.95 & 2.40 & 29.35 & 16 & 1 & 49 & 1 & 60.0 & 0.05 & 0.54 & 0.32 \\
\hline 60 & 13480 & 885 & 123 & 28.90 & 3.30 & 32.20 & 17 & 1 & 57 & 3 & 70.0 & 0.06 & 0.48 & 0.28 \\
\hline 70 & 15940 & 915 & 5700 & 35.40 & 2.85 & 38.25 & 20 & 3 & 68 & 2 & 63.0 & 0.04 & 0.51 & 0.29 \\
\hline
\end{tabular}

Note: Number of Time Periods $=9$, Number of Links $=20$, Number of Trucks $=8, \mathrm{WE}=1$ 


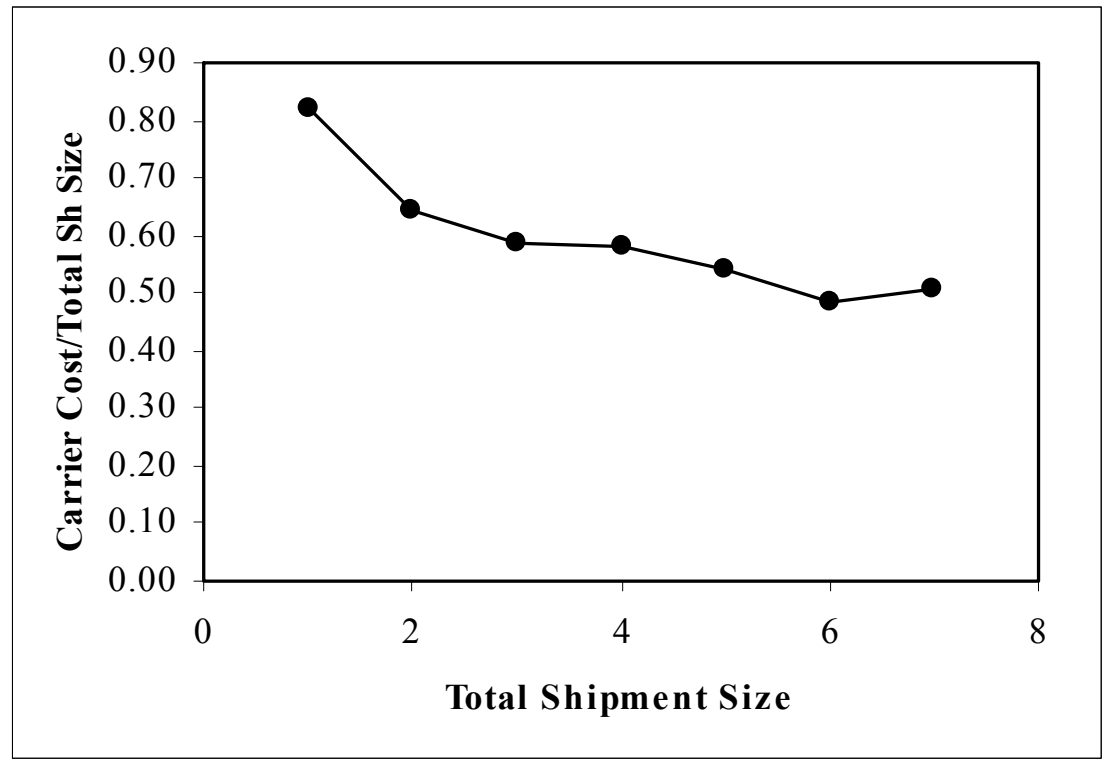

Figure 40 - Carrier's cost vs. Total shipment size (Case 3)

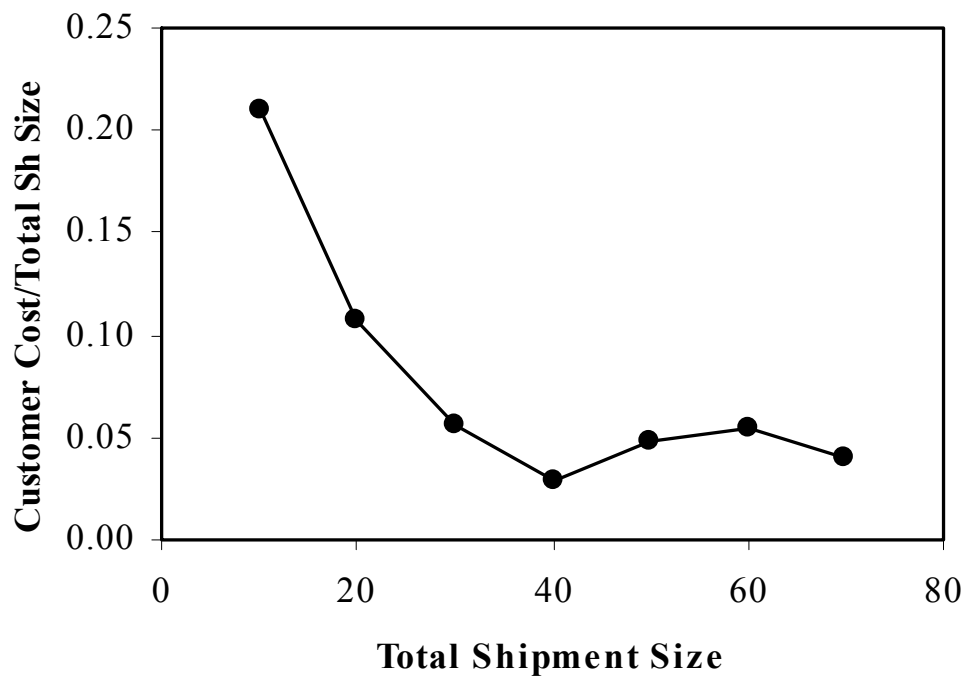

Figure 41 - Customers' cost vs. Total shipment cost (Case 3) 


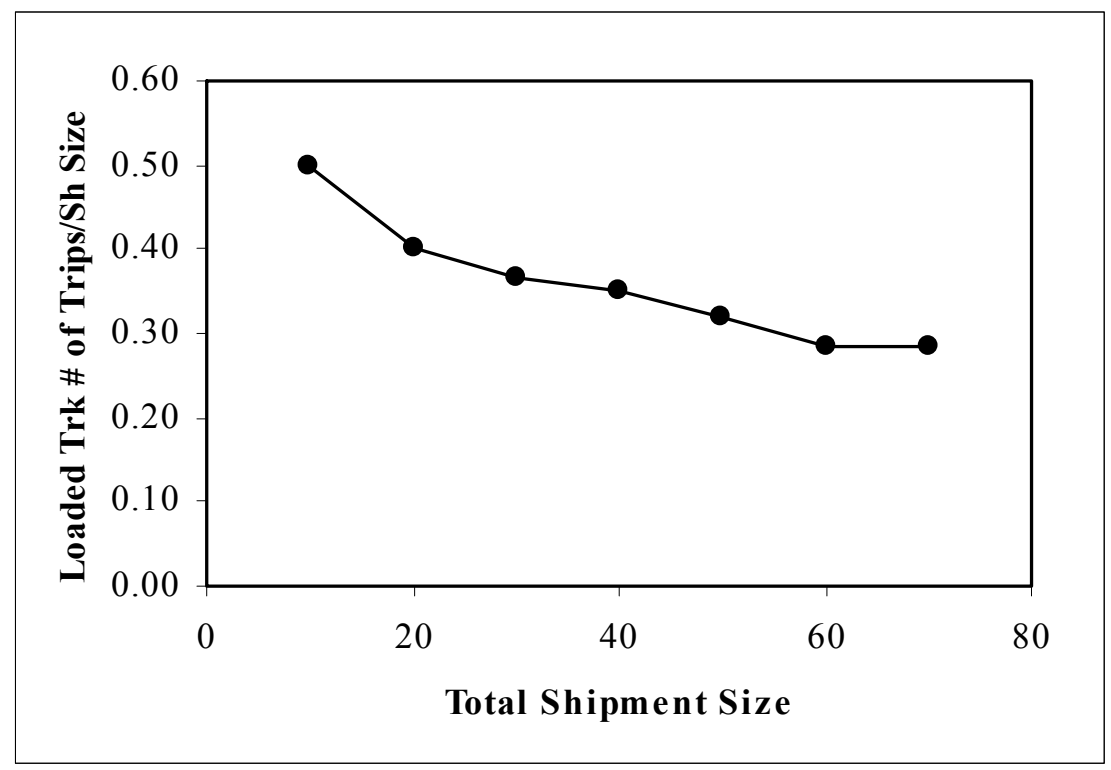

Figure 42 - Loaded truck number of trips vs. Total shipment size (Case 3)

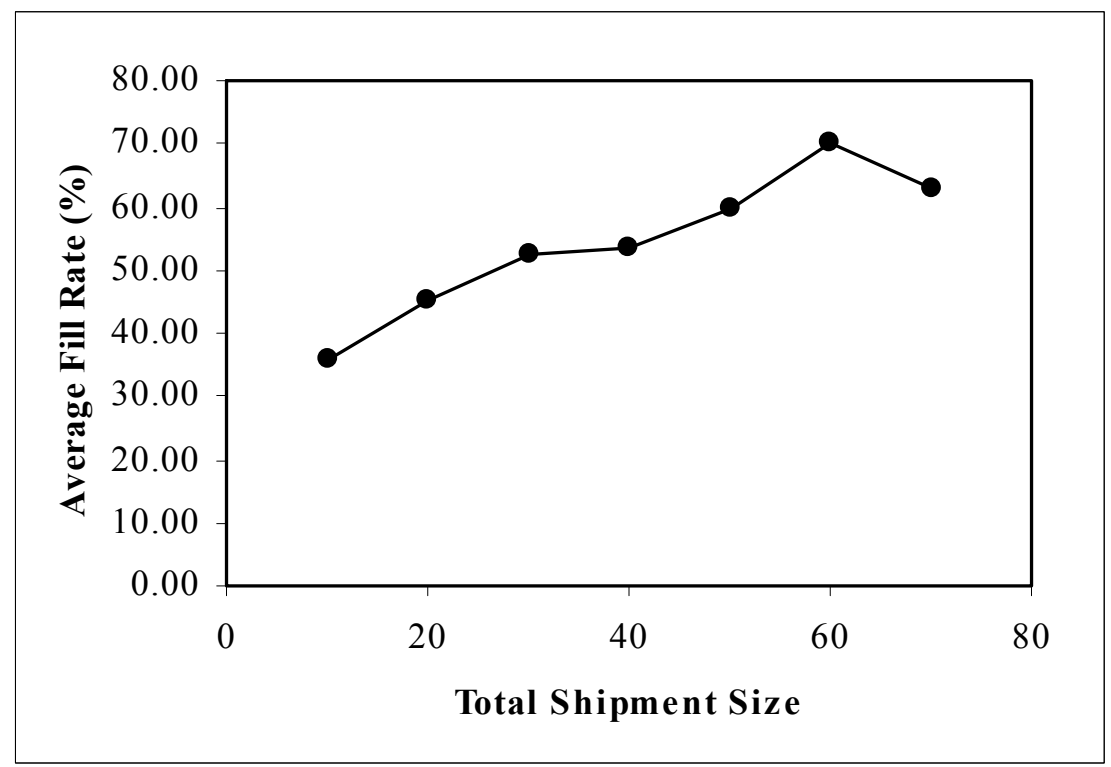

Figure 43 - Average fill rate vs. Total shipment size (Case 3) 


\section{Case 4: Variable Shipment Configuration}

This case focuses on the configuration of demand. Considering that all other parameters are fixed, 20 different instances of demand are generated randomly (using a uniform distribution). Table 12 presents the results. The first row is the so-called "base case", which is used for the previous sensitivity analyses. As shown in Table 12 , most of the performance measures do not show serious fluctuations with respect to changes in demand configuration. However, the execution time has a relatively high standard deviation. This means that the time required for CPLEX to find the optimal solution largely depends on the way that demand is distributed across the service area.

\section{Case 5: Variable Truck Locations}

The last case focuses on the location of empty trucks at the beginning of the operation. Considering that all other parameters are fixed, 20 different instances of truck locations are generated randomly (using a uniform distribution). The results are presented in Table 13. Similar to the last case, the first row is the "base case" that is used for the previous sensitivity analyses. As shown in Table 13, standard deviations are relatively low and most of the performance measures do not show fluctuations with respect to changes in the location of empty trucks. However, the execution time has a relatively high standard deviation. This means that the time required for CPLEX to find the optimal solution depends on the initial location of the empty trucks. 
Table 12 - Variable Shipment Configuration (Case 4)

\begin{tabular}{|c|c|c|c|c|c|c|c|c|c|c|c|}
\hline $\begin{array}{c}\text { Shipment } \\
\text { Configuration }\end{array}$ & $\begin{array}{l}\text { Number of } \\
\text { Variables }\end{array}$ & $\begin{array}{l}\text { Number of } \\
\text { Constraints }\end{array}$ & $\begin{array}{l}\text { Execution } \\
\text { Time (sec) }\end{array}$ & $\begin{array}{c}\text { Carrier } \\
\text { Cost } \\
\text { (unit) }\end{array}$ & $\begin{array}{l}\text { Customer } \\
\text { Cost (unit) }\end{array}$ & $\begin{array}{l}\text { Total } \\
\text { Cost } \\
\text { (unit) }\end{array}$ & $\begin{array}{c}\text { Loaded } \\
\text { Truck } \\
\text { Number } \\
\text { of Trips }\end{array}$ & $\begin{array}{c}\text { Empty } \\
\text { Truck } \\
\text { Number } \\
\text { of Trips }\end{array}$ & $\begin{array}{c}\text { Shipment } \\
\text { Delivered } \\
\text { (size) }\end{array}$ & $\begin{array}{c}\text { Shipment } \\
\text { Left } \\
\text { (size) }\end{array}$ & $\begin{array}{c}\text { Average } \\
\text { Fill Rate } \\
\quad(\%)\end{array}$ \\
\hline 01 (Base Case) & 9380 & 835 & 128 & 23.30 & 1.15 & 24.45 & 14 & 2 & 40 & 0 & 53.6 \\
\hline 02 & 8560 & 825 & 14 & 20.85 & 2.25 & 23.10 & 13 & 1 & 38 & 2 & 49.2 \\
\hline 03 & 9380 & 835 & 93 & 23.70 & 2.10 & 25.80 & 14 & 1 & 39 & 1 & 50.7 \\
\hline 04 & 8560 & 825 & 61 & 22.60 & 2.60 & 25.20 & 13 & 2 & 38 & 2 & 59.2 \\
\hline 05 & 9380 & 835 & 60 & 18.50 & 3.50 & 22.00 & 11 & 0 & 36 & 4 & 54.5 \\
\hline 06 & 9380 & 835 & 71 & 20.20 & 1.85 & 22.05 & 12 & 2 & 38 & 2 & 47.5 \\
\hline 07 & 8150 & 820 & 7 & 20.10 & 1.95 & 22.05 & 12 & 0 & 38 & 2 & 51.7 \\
\hline 08 & 9790 & 840 & 172 & 26.20 & 1.40 & 27.60 & 16 & 1 & 40 & 0 & 51.2 \\
\hline 09 & 7740 & 815 & 29 & 24.85 & 1.05 & 25.90 & 16 & 0 & 40 & 0 & 48.1 \\
\hline 10 & 8560 & 825 & 75 & 22.20 & 0.90 & 23.10 & 14 & 0 & 40 & 0 & 54.3 \\
\hline 11 & 9380 & 835 & 88 & 22.80 & 2.90 & 25.70 & 14 & 0 & 37 & 3 & 50.7 \\
\hline 12 & 8970 & 830 & 80 & 24.25 & 1.75 & 26.00 & 15 & 1 & 38 & 2 & 41.3 \\
\hline 13 & 8970 & 830 & 21 & 22.85 & 1.90 & 24.75 & 14 & 2 & 38 & 2 & 50.7 \\
\hline 15 & 8970 & 830 & 6 & 24.75 & 1.10 & 25.85 & 16 & 1 & 39 & 1 & 48.8 \\
\hline 16 & 8150 & 820 & 71 & 23.00 & 1.30 & 24.30 & 14 & 2 & 39 & 1 & 51.4 \\
\hline 17 & 8150 & 820 & 10 & 22.35 & 1.75 & 24.10 & 13 & 2 & 39 & 1 & 56.9 \\
\hline 18 & 9380 & 835 & 45 & 23.55 & 2.05 & 25.60 & 14 & 2 & 38 & 2 & 46.4 \\
\hline 19 & 8970 & 830 & 20 & 20.45 & 3.05 & 23.50 & 12 & 2 & 37 & 3 & 56.7 \\
\hline 20 & 8970 & 830 & 270 & 25.30 & 3.00 & 28.30 & 15 & 1 & 39 & 1 & 54.0 \\
\hline Average & 8909 & 829 & 67 & 22.62 & 1.98 & 24.60 & 13.7 & 1.2 & 38.5 & 1.6 & 51.7 \\
\hline Std. Dev. & 553 & 7 & 65 & 2.00 & 0.73 & 1.80 & 1.5 & 0.8 & 1.1 & 1.1 & 4.2 \\
\hline
\end{tabular}

Note: Number of Time Periods $=9$, Number of Trucks $=8$, Total Shipment Size $=40$, Number of Links $=20$, WE $=1$ 
Table 13 - Variable Truck Locations (Case 5)

\begin{tabular}{|c|c|c|c|c|c|c|c|c|c|c|c|}
\hline $\begin{array}{c}\text { Shipment } \\
\text { Configuration }\end{array}$ & $\begin{array}{l}\text { Number of } \\
\text { Variables }\end{array}$ & $\begin{array}{l}\text { Number of } \\
\text { Constraints }\end{array}$ & $\begin{array}{l}\text { Execution } \\
\text { Time (sec) }\end{array}$ & $\begin{array}{c}\text { Carrier } \\
\text { Cost } \\
\text { (unit) }\end{array}$ & $\begin{array}{l}\text { Customer } \\
\text { Cost (unit) }\end{array}$ & $\begin{array}{l}\text { Total } \\
\text { Cost } \\
\text { (unit) }\end{array}$ & $\begin{array}{c}\text { Loaded } \\
\text { Truck } \\
\text { Number } \\
\text { of Trips }\end{array}$ & $\begin{array}{c}\text { Empty } \\
\text { Truck } \\
\text { Number } \\
\text { of Trips }\end{array}$ & $\begin{array}{c}\text { Shipment } \\
\text { Delivered } \\
\text { (size) }\end{array}$ & $\begin{array}{c}\text { Shipment } \\
\text { Left } \\
\text { (size) }\end{array}$ & $\begin{array}{c}\text { Average } \\
\text { Fill Rate } \\
(\%)\end{array}$ \\
\hline 01 (Base Case) & 9380 & 835 & 128 & 23.30 & 1.15 & 24.45 & 14 & 2 & 40 & 0 & 53.6 \\
\hline 02 & 9380 & 835 & 68 & 22.00 & 2.10 & 24.10 & 13 & 1 & 38 & 2 & 56.9 \\
\hline 03 & 9380 & 835 & 162 & 22.15 & 3.15 & 25.30 & 13 & 3 & 36 & 4 & 56.2 \\
\hline 04 & 9380 & 835 & 128 & 22.40 & 3.30 & 25.70 & 13 & 3 & 36 & 4 & 54.6 \\
\hline 05 & 9380 & 835 & 51 & 22.50 & 2.70 & 25.20 & 13 & 3 & 37 & 3 & 56.9 \\
\hline 06 & 9380 & 835 & 48 & 23.30 & 1.15 & 24.45 & 14 & 2 & 40 & 0 & 53.6 \\
\hline 07 & 9380 & 835 & 87 & 23.10 & 2.00 & 25.10 & 14 & 1 & 38 & 2 & 52.9 \\
\hline 08 & 9380 & 835 & 86 & 22.60 & 2.70 & 25.30 & 13 & 2 & 37 & 3 & 56.2 \\
\hline 09 & 9380 & 835 & 114 & 22.20 & 3.35 & 25.55 & 13 & 4 & 36 & 4 & 56.9 \\
\hline 10 & 9380 & 835 & 112 & 21.75 & 2.70 & 24.45 & 13 & 0 & 37 & 3 & 56.9 \\
\hline 11 & 9380 & 835 & 49 & 22.65 & 2.90 & 25.55 & 13 & 3 & 37 & 3 & 56.2 \\
\hline 12 & 9380 & 835 & 98 & 21.90 & 2.95 & 24.85 & 13 & 3 & 36 & 4 & 54.6 \\
\hline 13 & 9380 & 835 & 137 & 22.65 & 2.85 & 25.50 & 13 & 3 & 37 & 3 & 54.6 \\
\hline 15 & 9380 & 835 & 42 & 22.10 & 1.40 & 23.50 & 14 & 0 & 39 & 1 & 53.6 \\
\hline 16 & 9380 & 835 & 199 & 22.10 & 3.65 & 25.75 & 12 & 3 & 38 & 4 & 61.7 \\
\hline 17 & 9380 & 835 & 82 & 23.75 & 1.95 & 25.70 & 14 & 2 & 39 & 1 & 53.6 \\
\hline 18 & 9380 & 835 & 36 & 22.60 & 1.85 & 24.45 & 13 & 2 & 39 & 1 & 56.9 \\
\hline 19 & 9380 & 835 & 41 & 21.70 & 2.95 & 24.65 & 13 & 2 & 36 & 4 & 54.6 \\
\hline 20 & 9380 & 835 & 151 & 22.70 & 3.20 & 25.90 & 13 & 3 & 37 & 3 & 54.6 \\
\hline Average & 9380 & 835 & 103 & 22.52 & 2.57 & 25.08 & 13.2 & 2.3 & 37.5 & 2.7 & 55.6 \\
\hline Std. Dev. & 0 & 0 & 55 & 0.55 & 0.76 & 0.69 & 0.5 & 1.1 & 1.4 & 1.4 & 2.0 \\
\hline
\end{tabular}

Note: Number of Time Periods $=9$, Number of Trucks $=8$, Total Shipment Size $=40$, Number of Links $=20$, WE $=1$ 


\section{Cases 6-10: Variable Shipment Cost Factors}

In this study, for the purpose of numerical experiments, it is assumed that a truck consumes one gallon of gas for every 100 ton-miles. Considering \$4 per gallon fuel cost, the total gas consumption of a full truck on each 600 mile trip is equal to $\$ 480$. The unit cost is defined to be equal to $\$ 400$. Therefore, the loaded truck routing cost is 1.2 units. Other cost factors that are associated with the shipments are selected based on some limited preliminary experiments. When the proposed decision making procedure and optimization methods are applied to a real world operation, all the shipments' associated costs must be calibrated to reflect the company's characteristics and also their attitude toward customer satisfaction. Case studies 6 through 10 show how changes in different cost factors may affect the system performance.

- Case 6 - Variable Shipment Handling Cost Factor (Table 14 and Figure 44, 45): The shipment handling cost is associated with the terminal operation and labor costs. As the handling cost factors increases, the company tends to deliver less shipment to be able to reduce the number of loaded truck movements.

- $\quad$ Case 7 - Variable Shipment Waiting Cost Factor (Table 15 and Figure 46, 47): The shipment waiting cost is associated with the costs of holding a shipment in a terminal. As the waiting cost factors increases, the number of loaded truck trips increases to avoid holding a shipment at intermediate breakbulks. As shown in Figure 47, by increasing the shipment waiting cost more trucks are operating to improve the delivery time. However, the number of shipments that are delivered remains constant. When the shipment waiting cost factor becomes equal to 0.3 all shipment can be delivered and there is an increase in average fill rate. Note that the optimization model does not intend to maximize the average fill rate; therefore any fluctuation in average fill rate would be acceptable.

- Case 8 - Variable Shipment Delivery Failure Cost Factor (Table 16 and Figure 48, 49): As the delivery failure cost factors increases, the company delivers as 
many shipments as possible by scheduling more loaded truck trips. This shows that the delivery failure cost is dominating the fuel costs.

- Case 9 - Variable Shipment Late Delivery Cost Factor (Table 17 and Figure $50,51)$ : In this case the early delivery becomes so crucial that the company would rather not to deliver those shipments that arrive late at their destinations. The number of truck movements remains the same, but with less shipment in each truck.

- Case 10 - Variable Shipment Early Delivery Cost Factor (Table 18 and Figure 52, 53): As discussed in Chapter 3 (Formulation Extension 3), an additional cost can be considered when there is a partial delivery for a shipment. In these cases, the carriers have to provide storage for that part of demand that is delivered earlier, so that the entire shipment for each customer can be delivered at once. This section shows how changes in early delivery cost factors may affect the system performance. As shown in Table 18 and Figure 52 , when the storage cost factor becomes equal to 0.25 the optimal solution would be obtained by reducing the number of shipments that are delivered. Hence there is a sudden drop in the value of objective function. Also when storage cost is equal to 0.35 , more loaded and empty truck movements are required in order to maintain the optimality of the solution. 
Table 14 - Shipment Handling Cost Factor (Case 6)

\begin{tabular}{|c|c|c|c|c|c|c|c|c|c|c|c|}
\hline $\begin{array}{c}\text { Shipment } \\
\text { Handling } \\
\text { Cost } \\
\text { Factor } \\
\end{array}$ & $\begin{array}{l}\text { Number of } \\
\text { Variables }\end{array}$ & $\begin{array}{l}\text { Number of } \\
\text { Constraints }\end{array}$ & $\begin{array}{l}\text { Execution } \\
\text { Time (sec) }\end{array}$ & $\begin{array}{c}\text { Carrier } \\
\text { Cost (unit) }\end{array}$ & $\begin{array}{l}\text { Customer } \\
\text { Cost (unit) }\end{array}$ & $\begin{array}{c}\text { Total } \\
\text { Cost (unit) }\end{array}$ & $\begin{array}{c}\text { Loaded } \\
\text { Truck } \\
\text { Number } \\
\text { of Trips } \\
\end{array}$ & $\begin{array}{c}\text { Empty } \\
\text { Truck } \\
\text { Number } \\
\text { of Trips } \\
\end{array}$ & $\begin{array}{c}\text { Shipment } \\
\text { Delivered } \\
\text { (size) }\end{array}$ & $\begin{array}{c}\text { Shipment } \\
\text { Left } \\
\text { (size) }\end{array}$ & $\begin{array}{c}\text { Average } \\
\text { Fill Rate } \\
(\%)\end{array}$ \\
\hline 0.05 & 9380 & 835 & 128 & 23.30 & 1.15 & 24.45 & 14 & 2 & 40 & 0 & 53.60 \\
\hline 0.10 & 9380 & 835 & 34 & 26.05 & 2.10 & 28.15 & 13 & 2 & 38 & 2 & 56.90 \\
\hline 0.15 & 9380 & 835 & 56 & 29.10 & 2.55 & 31.65 & 13 & 2 & 37 & 3 & 53.80 \\
\hline 0.20 & 9380 & 835 & 54 & 30.90 & 4.05 & 34.95 & 12 & 3 & 34 & 6 & 53.30 \\
\hline 0.25 & 9380 & 835 & 31 & 31.10 & 6.60 & 37.70 & 11 & 3 & 32 & 8 & 47.30 \\
\hline 0.30 & 9380 & 835 & 13 & 33.70 & 6.60 & 40.30 & 11 & 3 & 32 & 8 & 47.30 \\
\hline 0.35 & 9380 & 835 & 10 & 36.30 & 6.60 & 42.90 & 11 & 3 & 32 & 8 & 47.30 \\
\hline
\end{tabular}

Note: Number of Time Periods $=9$, Number of Links $=20$, Number of Trucks $=8$, Total Shipment Size $=40$, WE $=1$ 


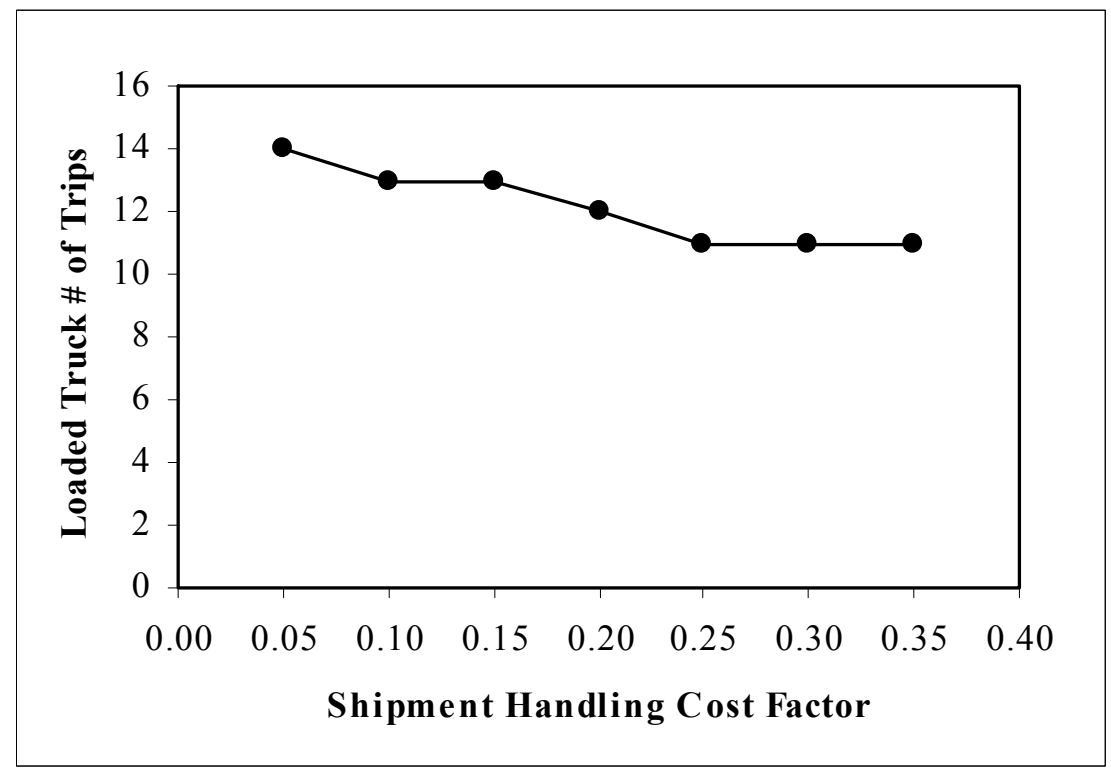

Figure 44 - Loaded truck \# of Trips vs. Handling cost factor (Case 6)

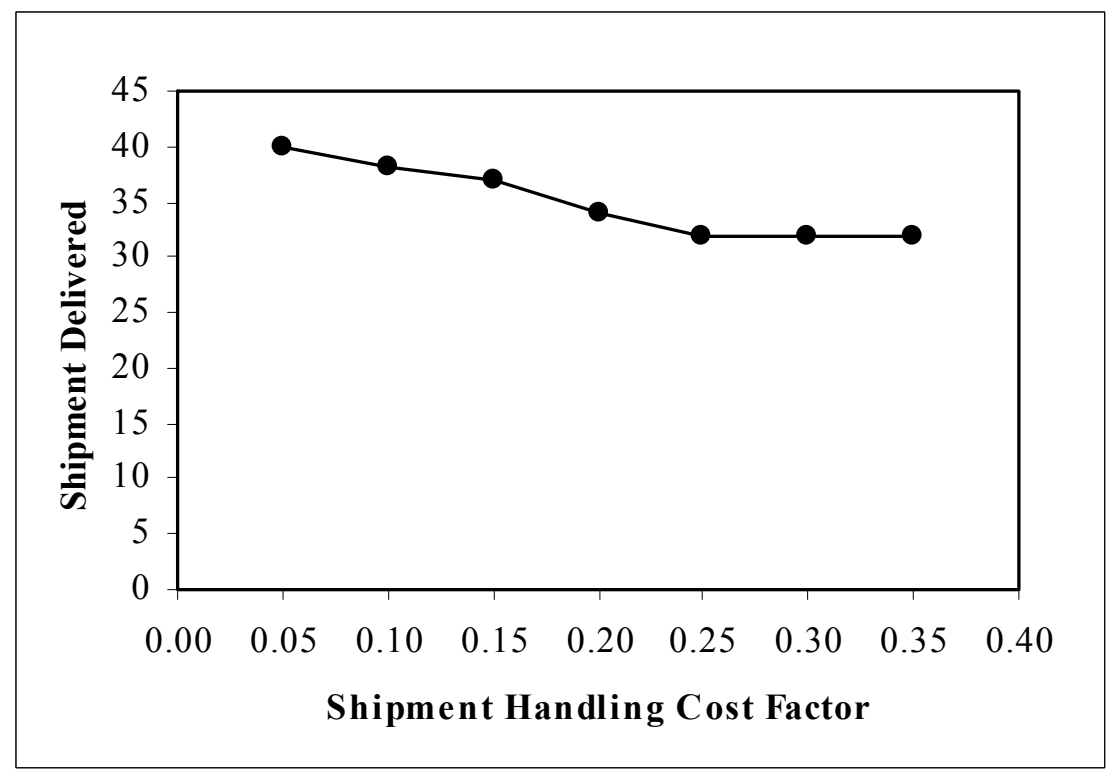

Figure 45 - Shipment delivered vs. Handling cost factor (Case 6) 
Table 15 - Shipment Waiting Cost Factor (Case 7)

\begin{tabular}{|c|c|c|c|c|c|c|c|c|c|c|c|}
\hline $\begin{array}{c}\text { Shipment } \\
\text { Waiting } \\
\text { Cost } \\
\text { Factor } \\
\end{array}$ & $\begin{array}{l}\text { Number of } \\
\text { Variables }\end{array}$ & $\begin{array}{l}\text { Number of } \\
\text { Constraints }\end{array}$ & $\begin{array}{l}\text { Execution } \\
\text { Time (sec) }\end{array}$ & $\begin{array}{c}\text { Carrier } \\
\text { Cost (unit) }\end{array}$ & $\begin{array}{l}\text { Customer } \\
\text { Cost (unit) }\end{array}$ & $\begin{array}{c}\text { Total } \\
\text { Cost (unit) }\end{array}$ & $\begin{array}{c}\text { Loaded } \\
\text { Truck } \\
\text { Number } \\
\text { of Trips } \\
\end{array}$ & $\begin{array}{c}\text { Empty } \\
\text { Truck } \\
\text { Number } \\
\text { of Trips } \\
\end{array}$ & $\begin{array}{c}\text { Shipment } \\
\text { Delivered } \\
\text { (size) }\end{array}$ & $\begin{array}{c}\text { Shipment } \\
\text { Left } \\
\text { (size) }\end{array}$ & $\begin{array}{c}\text { Average } \\
\text { Fill Rate } \\
\text { (\%) }\end{array}$ \\
\hline 0.05 & 9380 & 835 & 128 & 23.30 & 1.15 & 24.45 & 14 & 2 & 40 & 0 & 53.60 \\
\hline 0.10 & 9380 & 835 & 142 & 25.15 & 1.40 & 26.55 & 14 & 2 & 39 & 1 & 53.60 \\
\hline 0.15 & 9380 & 835 & 191 & 26.90 & 1.35 & 28.25 & 15 & 2 & 39 & 1 & 49.30 \\
\hline 0.20 & 9380 & 835 & 253 & 28.40 & 1.30 & 29.70 & 16 & 2 & 39 & 1 & 47.50 \\
\hline 0.25 & 9380 & 835 & 104 & 29.65 & 1.30 & 30.95 & 16 & 2 & 39 & 1 & 47.50 \\
\hline 0.30 & 9380 & 835 & 166 & 31.00 & 1.00 & 32.00 & 17 & 2 & 40 & 0 & 49.40 \\
\hline 0.35 & 9380 & 835 & 131 & 31.65 & 1.35 & 33.00 & 17 & 2 & 40 & 0 & 53.50 \\
\hline
\end{tabular}

Note: Number of Time Periods $=9$, Number of Links $=20$, Number of Trucks $=8$, Total Shipment Size $=40$, WE $=1$ 


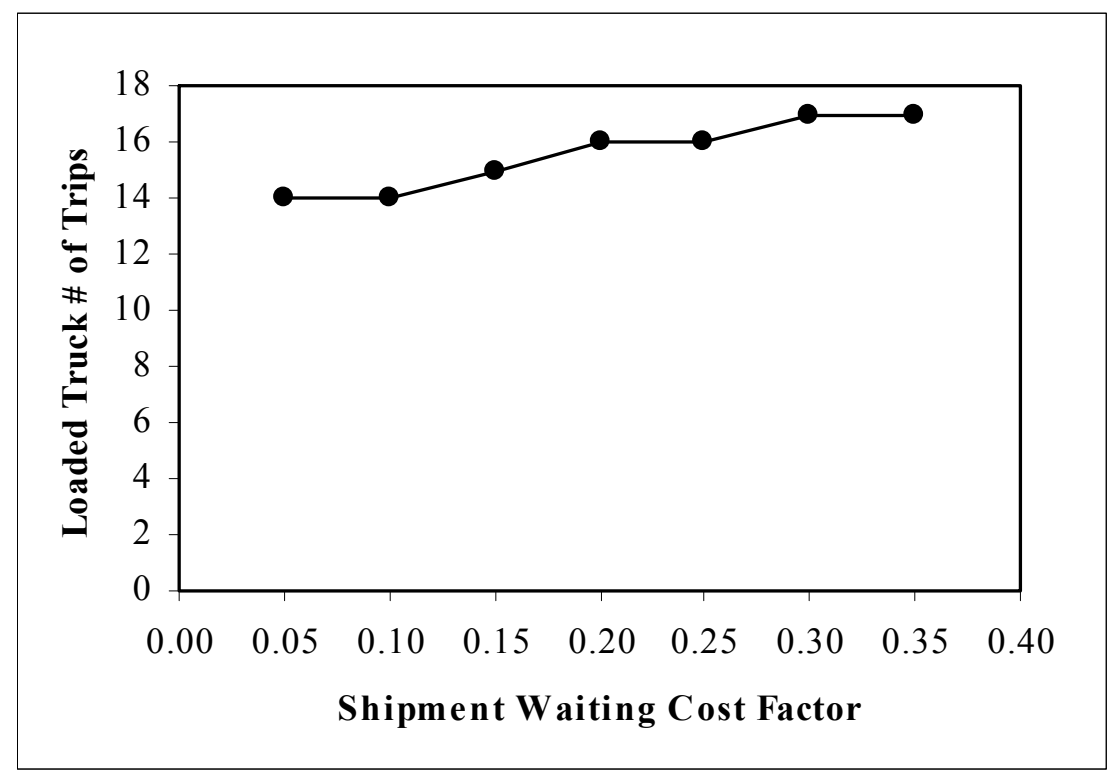

Figure 46 - Loaded truck \# of trips vs. Waiting cost factor (Case 7)

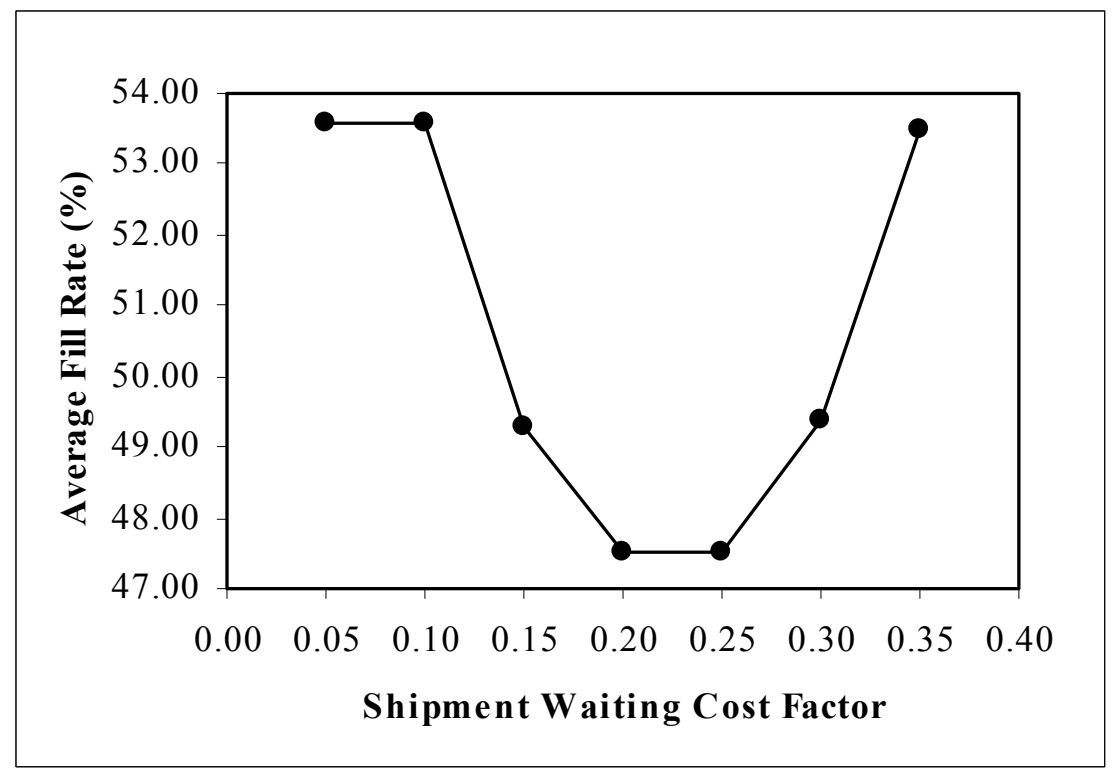

Figure 47 - Avg. fill rate vs. Waiting cost factor (Case 7) 
Table 16 - Shipment Delivery Failure Cost Factor (Case 8)

\begin{tabular}{|c|c|c|c|c|c|c|c|c|c|c|c|}
\hline $\begin{array}{l}\text { Shipment } \\
\text { Delivery } \\
\text { Failure } \\
\text { Cost Factor } \\
\end{array}$ & $\begin{array}{l}\text { Number of } \\
\text { Variables }\end{array}$ & $\begin{array}{l}\text { Number of } \\
\text { Constraints }\end{array}$ & $\begin{array}{l}\text { Execution } \\
\text { Time (sec) }\end{array}$ & $\begin{array}{c}\text { Carrier } \\
\text { Cost (unit) }\end{array}$ & $\begin{array}{l}\text { Customer } \\
\text { Cost (unit) }\end{array}$ & $\begin{array}{c}\text { Total } \\
\text { Cost (unit) }\end{array}$ & $\begin{array}{c}\text { Loaded } \\
\text { Truck } \\
\text { Number } \\
\text { of Trips } \\
\end{array}$ & $\begin{array}{c}\text { Empty } \\
\text { Truck } \\
\text { Number } \\
\text { of Trips } \\
\end{array}$ & $\begin{array}{c}\text { Shipment } \\
\text { Delivered } \\
\text { (size) }\end{array}$ & $\begin{array}{c}\text { Shipment } \\
\text { Left } \\
\text { (size) }\end{array}$ & $\begin{array}{c}\text { Average } \\
\text { Fill Rate } \\
\text { (\%) }\end{array}$ \\
\hline 0.05 & 9380 & 835 & 17 & 18.85 & 2.15 & 21.00 & 8 & 0 & 25 & 15 & 70.00 \\
\hline 0.10 & 9380 & 835 & 27 & 19.50 & 2.75 & 22.25 & 10 & 2 & 31 & 9 & 63.00 \\
\hline 0.15 & 9380 & 835 & 71 & 20.25 & 2.85 & 23.10 & 11 & 2 & 32 & 8 & 62.70 \\
\hline 0.20 & 9380 & 835 & 179 & 21.10 & 2.65 & 23.75 & 12 & 2 & 35 & 5 & 60.00 \\
\hline 0.25 & 9380 & 835 & 129 & 22.35 & 1.90 & 24.25 & 13 & 2 & 38 & 2 & 56.90 \\
\hline 0.30 & 9380 & 835 & 128 & 23.30 & 1.15 & 24.45 & 14 & 2 & 40 & 0 & 53.60 \\
\hline 0.35 & 9380 & 835 & 33 & 23.30 & 1.15 & 24.45 & 14 & 2 & 40 & 0 & 53.60 \\
\hline
\end{tabular}

Note: Number of Time Periods $=9$, Number of Links $=20$, Number of Trucks $=8$, Total Shipment Size $=40$, WE $=1$ 


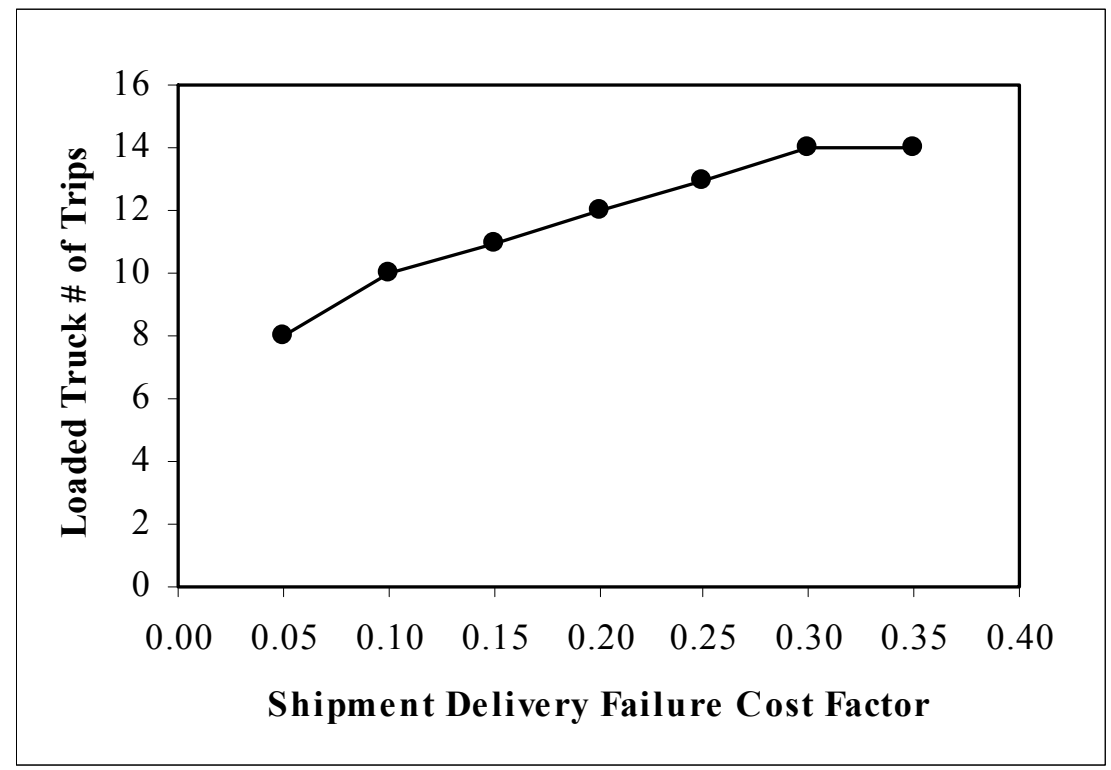

Figure 48 - Loaded truck \# of trips vs. Delivery failure cost factor (Case 8)

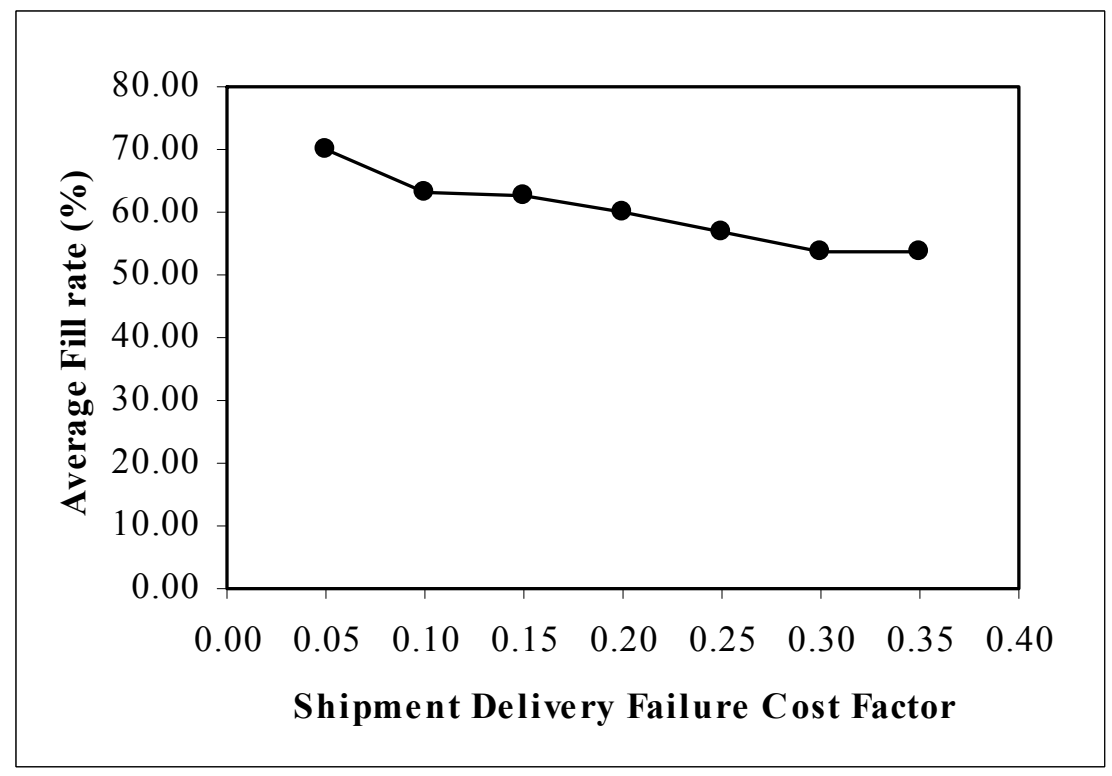

Figure 49 - Avg. fill rate vs. Delivery failure cost factor (Case 8) 
Table 17 - Shipment Late Delivery Cost Factor (Case 9)

\begin{tabular}{|c|c|c|c|c|c|c|c|c|c|c|c|}
\hline $\begin{array}{l}\text { Shipment } \\
\text { Late } \\
\text { Delivery } \\
\text { Cost Factor } \\
\end{array}$ & $\begin{array}{l}\text { Number of } \\
\text { Variables }\end{array}$ & $\begin{array}{l}\text { Number of } \\
\text { Constraints }\end{array}$ & $\begin{array}{l}\text { Execution } \\
\text { Time (sec) }\end{array}$ & $\begin{array}{c}\text { Carrier } \\
\text { Cost (unit) }\end{array}$ & $\begin{array}{l}\text { Customer } \\
\text { Cost (unit) }\end{array}$ & $\begin{array}{c}\text { Total } \\
\text { Cost (unit) }\end{array}$ & $\begin{array}{c}\text { Loaded } \\
\text { Truck } \\
\text { Number } \\
\text { of Trips } \\
\end{array}$ & $\begin{array}{c}\text { Empty } \\
\text { Truck } \\
\text { Number } \\
\text { of Trips } \\
\end{array}$ & $\begin{array}{l}\text { Shipment } \\
\text { Delivered } \\
\text { (size) }\end{array}$ & $\begin{array}{c}\text { Shipment } \\
\text { Left } \\
\text { (size) }\end{array}$ & $\begin{array}{c}\text { Average } \\
\text { Fill Rate } \\
(\%)\end{array}$ \\
\hline 0.05 & 9380 & 835 & 128 & 23.30 & 1.15 & 24.45 & 14 & 2 & 40 & 0 & 53.60 \\
\hline 0.10 & 9380 & 835 & 56 & 23.05 & 2.20 & 25.25 & 14 & 2 & 39 & 1 & 53.60 \\
\hline 0.15 & 9380 & 835 & 31 & 23.00 & 2.85 & 25.85 & 14 & 2 & 38 & 2 & 50.70 \\
\hline 0.20 & 9380 & 835 & 56 & 22.60 & 3.60 & 26.20 & 14 & 2 & 36 & 4 & 50.00 \\
\hline 0.25 & 9380 & 835 & 21 & 23.30 & 3.00 & 26.30 & 14 & 3 & 35 & 5 & 45.00 \\
\hline 0.30 & 9380 & 835 & 21 & 23.30 & 3.00 & 26.30 & 14 & 3 & 35 & 5 & 45.00 \\
\hline 0.35 & 9380 & 835 & 52 & 23.30 & 3.00 & 26.30 & 14 & 3 & 35 & 5 & 45.00 \\
\hline
\end{tabular}

Note: Number of Time Periods $=9$, Number of Links $=20$, Number of Trucks $=8$, Total Shipment Size $=40$, WE $=1$ 


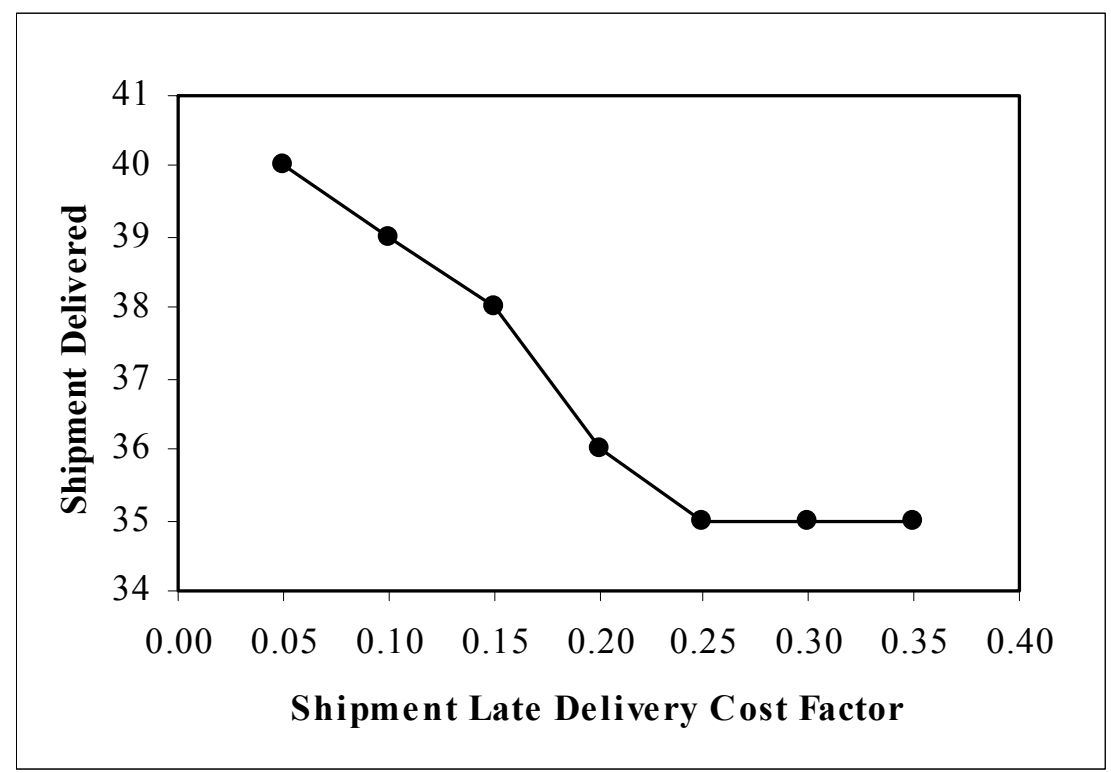

Figure 50 - Shipment delivered vs. Late-delivery cost factor (Case 9)

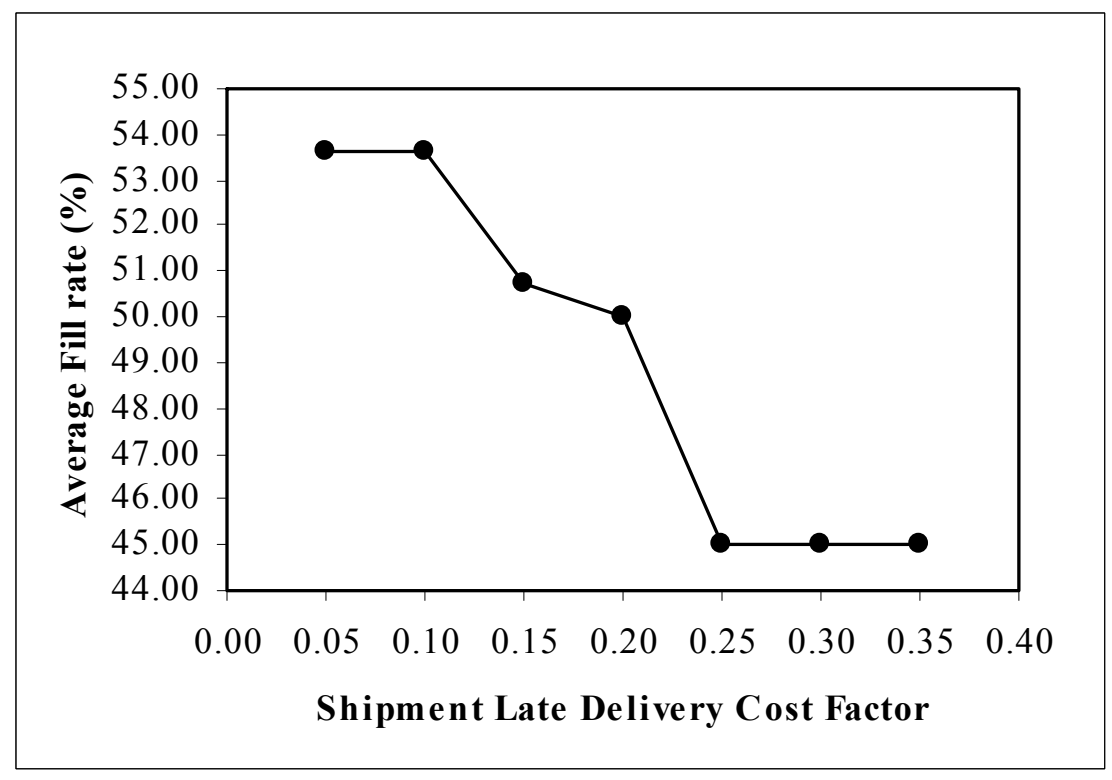

Figure $51-$ Avg. fill rate vs. Late-delivery cost factor (Case 9) 
Table 18 - Shipment Early Delivery (Storage) Cost Factor (Case 10)

\begin{tabular}{|c|c|c|c|c|c|c|c|c|c|c|c|}
\hline $\begin{array}{l}\text { Shipment } \\
\text { Late } \\
\text { Delivery } \\
\text { Cost Factor } \\
\end{array}$ & $\begin{array}{l}\text { Number of } \\
\text { Variables }\end{array}$ & $\begin{array}{l}\text { Number of } \\
\text { Constraints }\end{array}$ & $\begin{array}{l}\text { Execution } \\
\text { Time (sec) }\end{array}$ & $\begin{array}{c}\text { Carrier } \\
\text { Cost (unit) }\end{array}$ & $\begin{array}{l}\text { Customer } \\
\text { Cost (unit) }\end{array}$ & $\begin{array}{c}\text { Total } \\
\text { Cost (unit) }\end{array}$ & $\begin{array}{c}\text { Loaded } \\
\text { Truck } \\
\text { Number } \\
\text { of Trips } \\
\end{array}$ & $\begin{array}{c}\text { Empty } \\
\text { Truck } \\
\text { Number } \\
\text { of Trips } \\
\end{array}$ & $\begin{array}{c}\text { Shipment } \\
\text { Delivered } \\
\text { (size) }\end{array}$ & $\begin{array}{c}\text { Shipment } \\
\text { Left } \\
\text { (size) }\end{array}$ & $\begin{array}{c}\text { Average } \\
\text { Fill Rate } \\
\quad(\%)\end{array}$ \\
\hline 0.05 & 9380 & 835 & 50 & 23.75 & 1.85 & 25.60 & 13 & 2 & 39 & 1 & 56.90 \\
\hline 0.10 & 9380 & 835 & 58 & 24.60 & 1.85 & 26.45 & 13 & 2 & 39 & 1 & 56.90 \\
\hline 0.15 & 9380 & 835 & 39 & 25.45 & 1.85 & 27.30 & 13 & 2 & 39 & 1 & 56.90 \\
\hline 0.20 & 9380 & 835 & 177 & 26.30 & 1.85 & 28.15 & 13 & 2 & 39 & 1 & 56.90 \\
\hline 0.25 & 9380 & 835 & 672 & 25.65 & 3.25 & 28.90 & 13 & 2 & 37 & 3 & 55.40 \\
\hline 0.30 & 9380 & 835 & 471 & 25.95 & 3.25 & 29.20 & 13 & 2 & 37 & 3 & 55.40 \\
\hline 0.35 & 9380 & 835 & 1017 & 26.55 & 2.75 & 29.30 & 15 & 3 & 38 & 2 & 57.30 \\
\hline
\end{tabular}

Note: Number of Time Periods $=9$, Number of Links $=20$, Number of Trucks $=8$, Total Shipment Size $=40$, WE $=1$ 


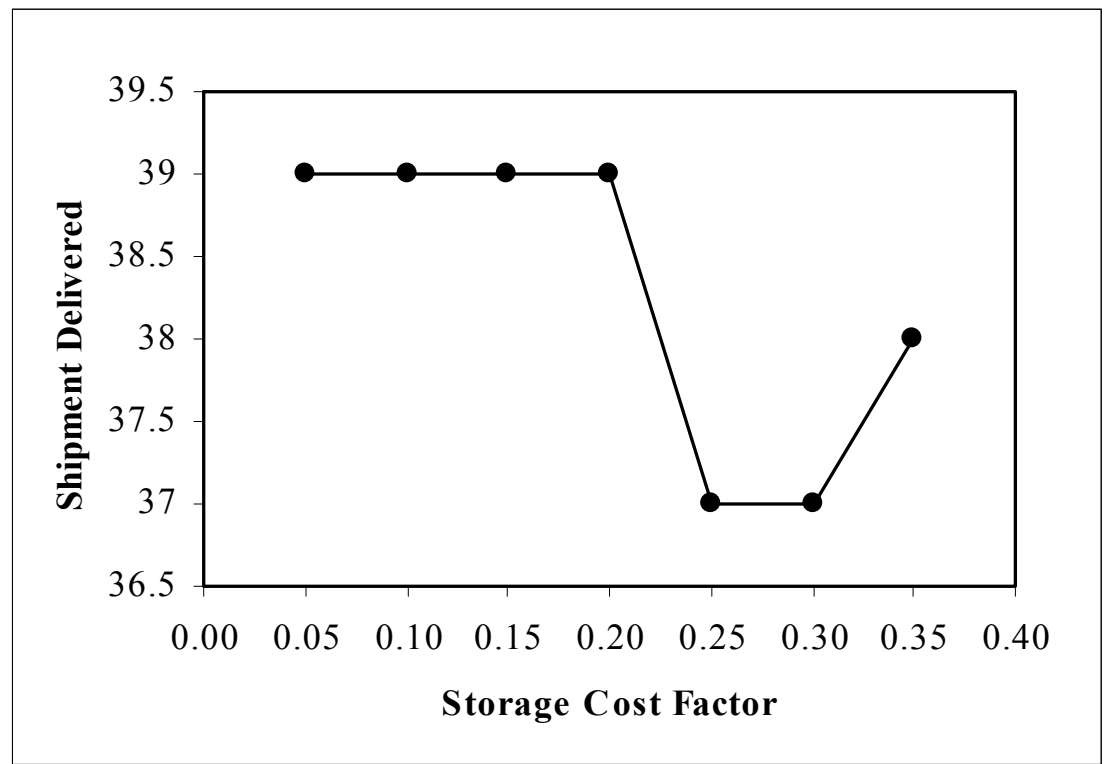

Figure 52 - Shipment delivered vs. Storage cost factor (Case 10)

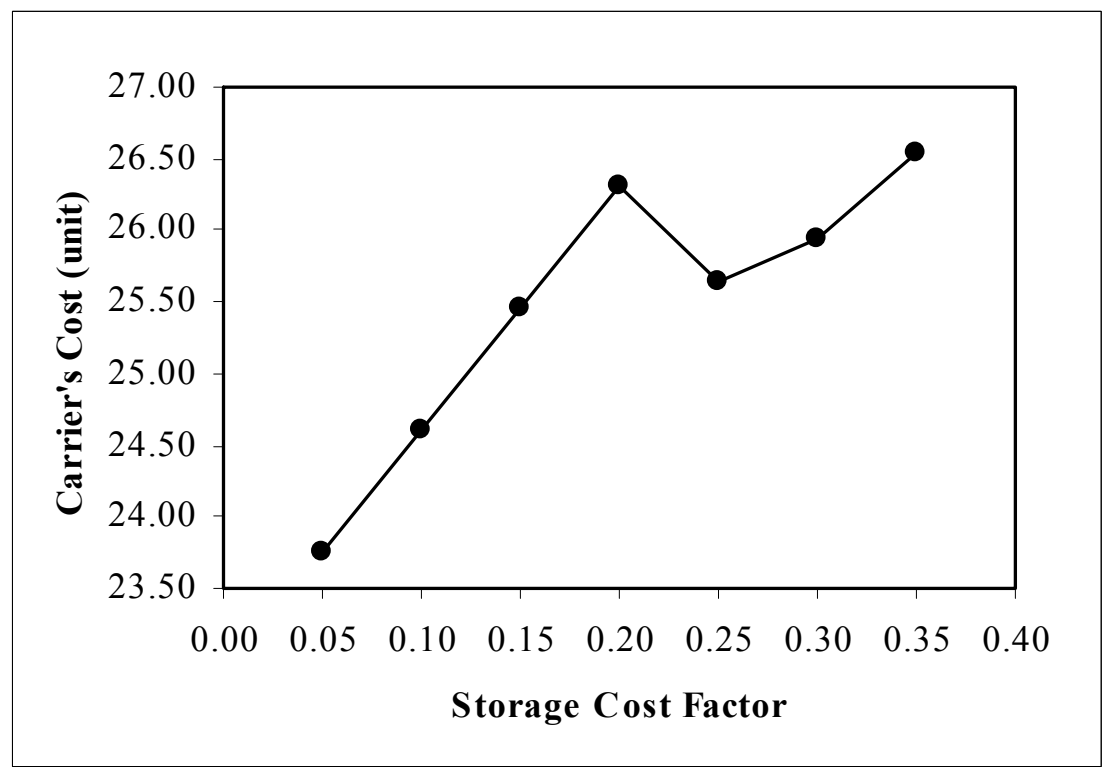

Figure 53 - Carrier's cost vs. Storage cost factor (Case 10) 


\section{- Driver Routing}

In Chapter 3, as an extension to the original mathematical model, the driver routing and scheduling problem was combined with truck/shipment routing and scheduling problem. The required additions to assumptions, notations, objective function and constraints of the original mathematical formulation were addressed. The numerical experiments were conducted using the 10-terminal network. This section presents the results of these experiments and discusses the impact of driver routing extension on the size of the optimization model, and the computation time that is required to solve the problem.

In the base case of the 10-terminal problem, 8 trucks are available at to deliver 40 shipments. It is assumed that at time 0 there is one driver for each empty truck. All the other characteristics of the problem are the same as the original problem.

To generate the mathematical problem for problem with driver routing, the "Problem Generator" C program is modified by adding the additional cost function and constraints. A new input file is added that contains the information related to each driver; the identification number, location, home and the associated cost factors. Problem characteristics are shown in Figure 54 and 55.

The "Operation Plan" C program is also modified. Additional processed output files are added that show the driver dispatch plan at each terminal and driver routing plan for each driver. Problem is solved by CPLEX and the performance measures are presented in Figure 56 and 57. The samples of new driver related output files are shown in Figure 58 and 59.

Using CPLEX the optimal solution can be obtained for the original problem (without driver routing) in 128 seconds. However, the problem with driver routing is solved in 29,112 seconds ( $~ 8$ hours) using CPLEX. As shown in Figure 55, considering the driver routing problem would add 6,000 variables and 2,200 more constraints to the MIP problem which results in an exponentially increased execution time. 


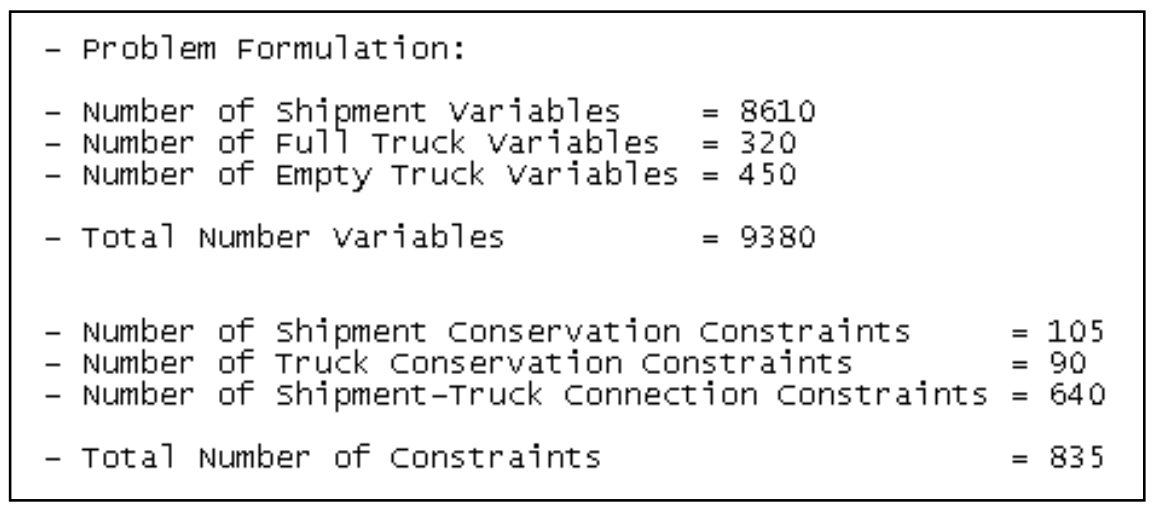

Figure 54 - Problem characteristics (without driver routing)

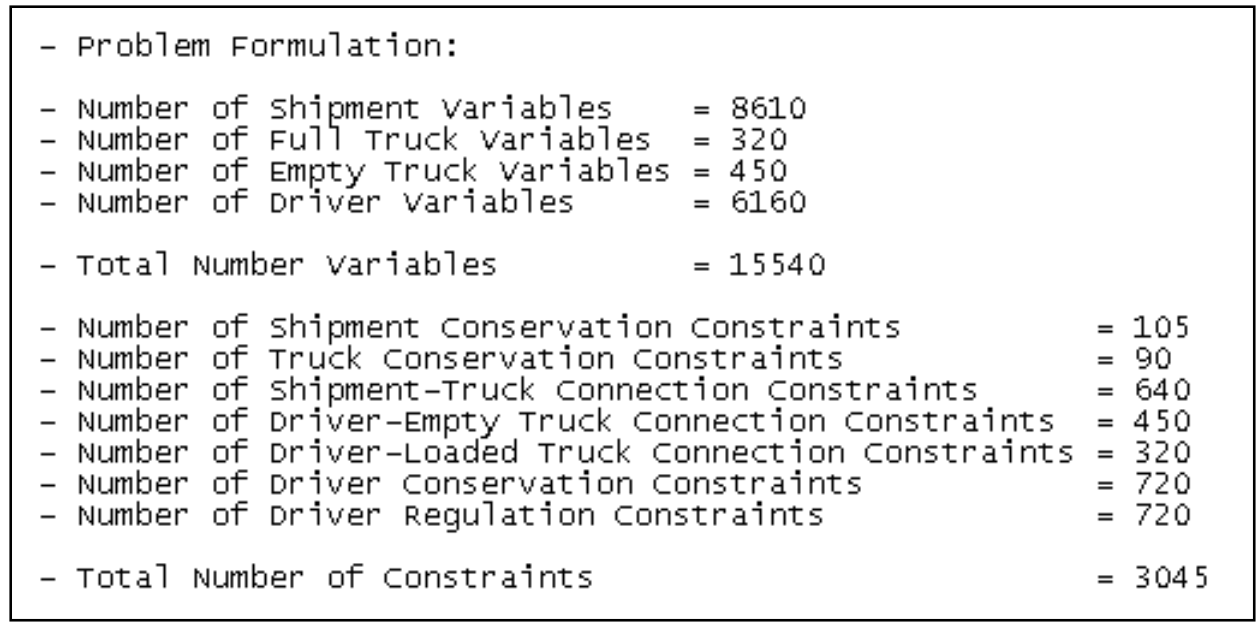

Figure 55 - Problem characteristics (with driver routing) 


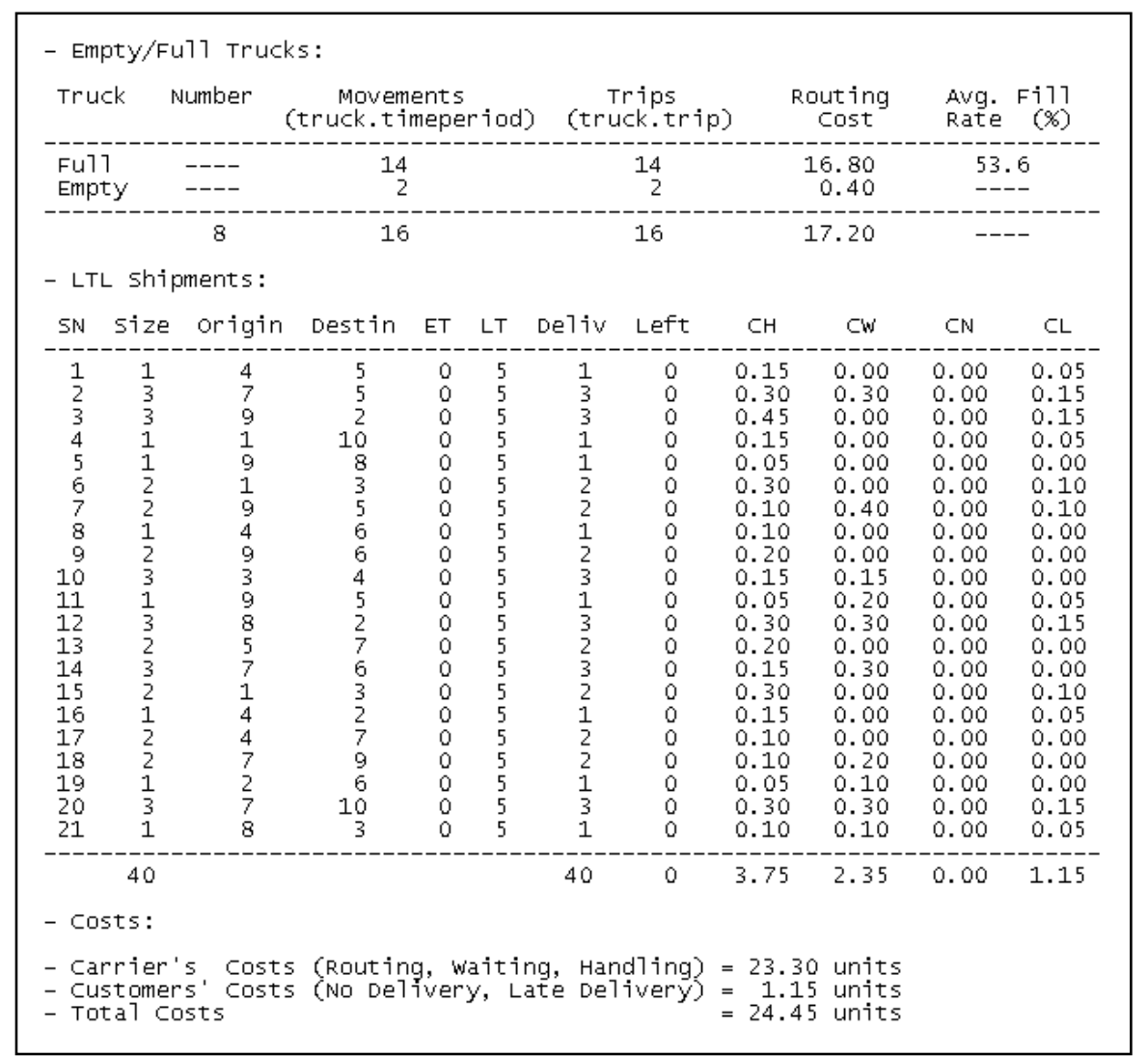

Figure 56 - Performance measures (without driver routing)

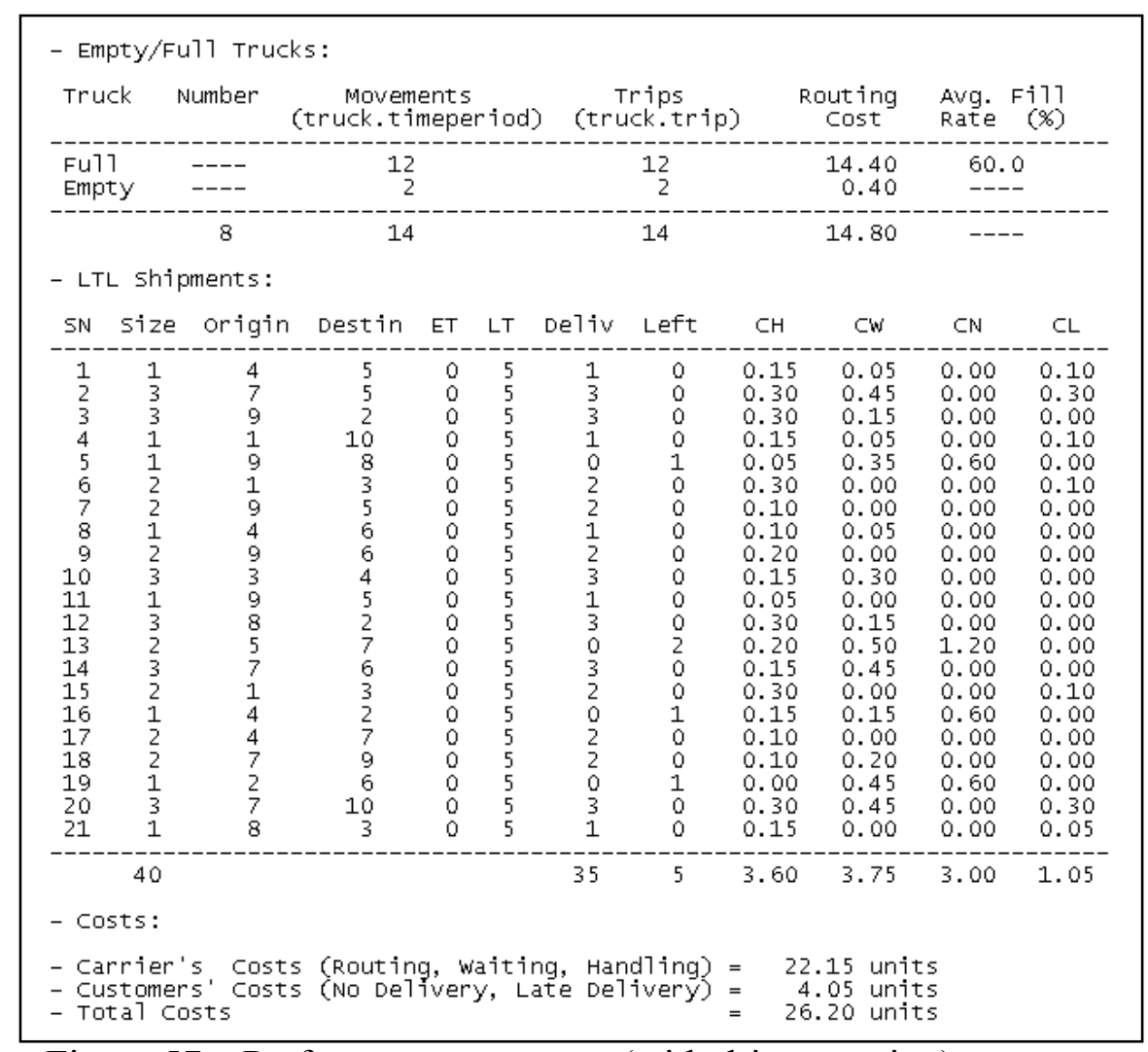

Figure 57 - Performance measures (with driver routing) 


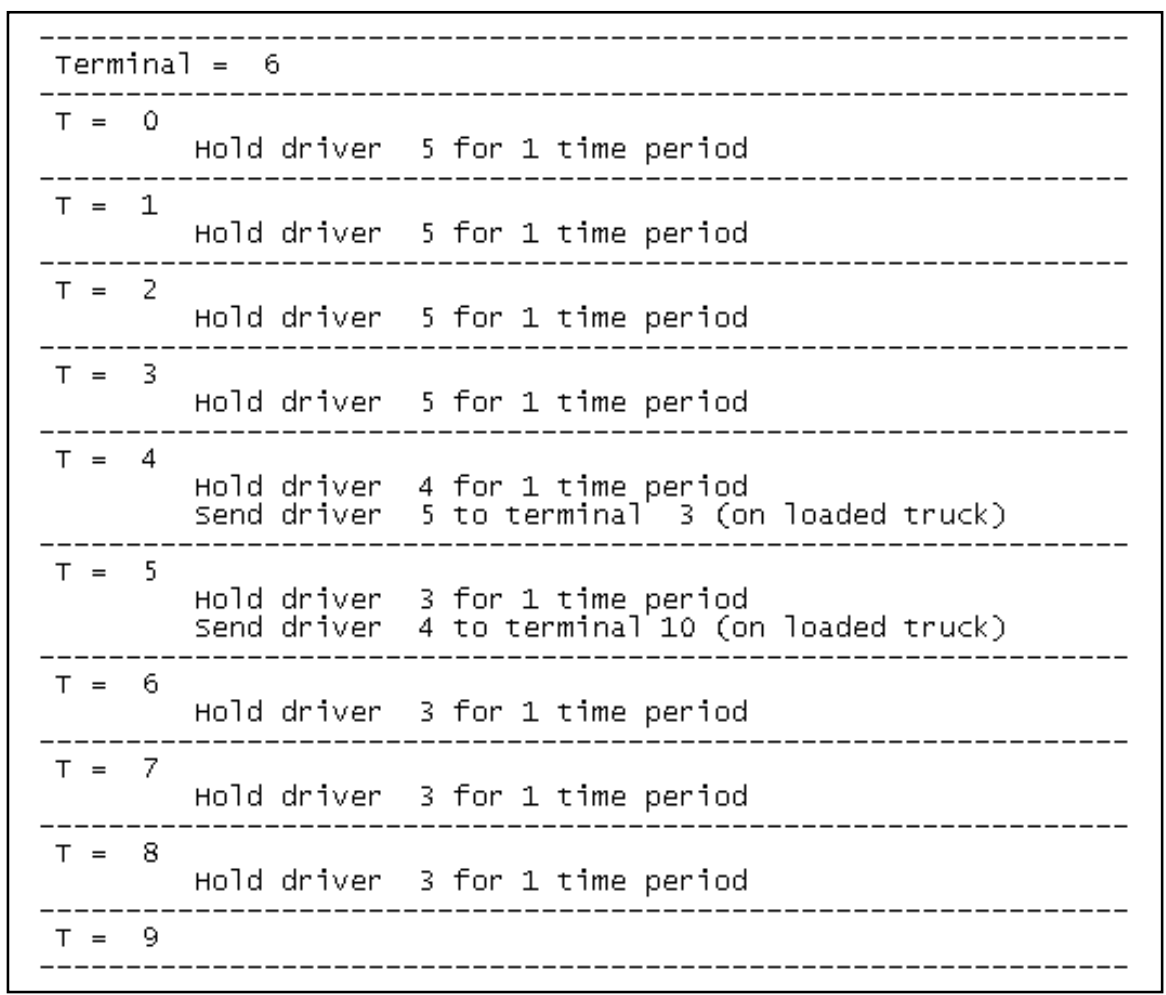

Figure 58 - Driver dispatch plan at terminal 6

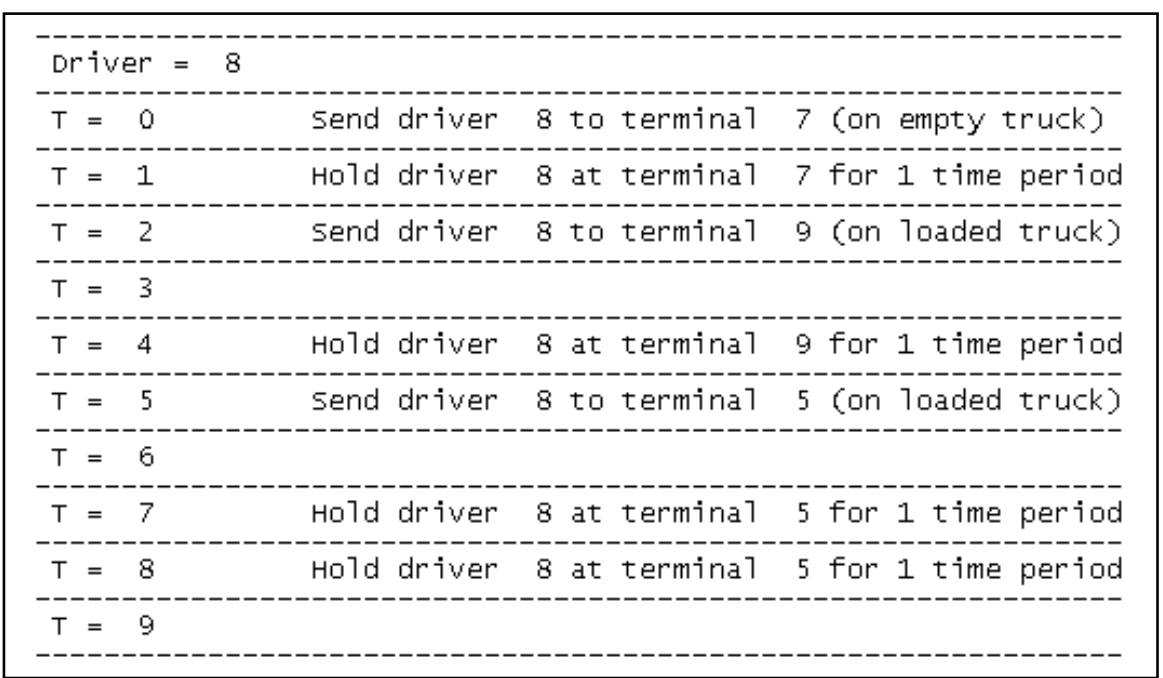

Figure 59 - Driver routing plan for driver 8 


\section{Chapter 6: Solution Algorithms}

In the Chapter 4 different procedures were proposed to make acceptance/rejection decisions for LTL motor carriers. One major step in all the proposed procedures is to solve the shipment and truck routing problem, repeatedly. When the size of the problem is large, one cannot rely on the exact solution methods (i.e. CPLEX) to solve the MIP and obtain the solution in a timely manner. Therefore under high demand for LTL shipments it is not practical to use CPLEX. One way to resolve this issue is to solve the MIP using heuristic approaches that are the main subjects of this Chapter.

In this study, three different solution algorithms are proposed to solve the MIP problem. These approaches are presented in the same order that they were developed during this research. The first 2 approaches are based on a search algorithm that tries to find the best paths to route the shipments. The last approach uses a graphic partitioning to reduce the size of the problem that is solved using CPLEX. The development of the first two solution algorithm led us to come up with the idea of path-based network partitioning that is used in the $3^{\text {rd }}$ approach. In all 3 algorithms, instead of working with link variables, shipments and trucks routing paths are the main variables. That approach helps us to reduce the number of variables and the size of search space significantly.

In order to evaluate the effectiveness of the solution algorithms, three benchmark solutions are proposed. The first two benchmarks are "do-nothing" and "literature-reported operation", which represent the upper bound or the worst possible solutions. Obviously, all proposed solution algorithms must perform better than these benchmarks. The last benchmark is the lower bound that is derived using a relaxation method. The lower bound will be used particularly to determine the quality of the solution provided by the network partitioning method, which is the $3^{\text {rd }}$ proposed solution algorithm. In this section, the step by step procedure shows how the benchmarks and heuristic solutions are developed. Next Chapter will focus on analyzing the performance of the proposed methods. 


\section{Benchmark 1: Do-Nothing}

Naturally, the do-nothing solution would be the first choice to be used as a benchmark to evaluate the performance of the solution. The do-nothing solution is considered to have the worst possible approach to handle the demand. Clearly, any other approach that performs worse than do-nothing would not be considered as a candidate to solve the optimization problem.

To generate the do-nothing solution, it is assumed that all shipments stay at their origin terminals and there is no delivery. There will be no loaded or empty truck movements. Waiting costs and delivery failure costs will be the only costs that are associated with this solution. In order to calculate the objective function for donothing solution a $\mathrm{C}$ program was developed. The program uses the shipment data as input and calculates the objective function by adding waiting and delivery failure costs. The outputs follow the CPLEX format, so by running the "operation plan" program the performance measures are generated and used as a benchmark to compare with the solution provided by the proposed heuristic algorithms.

\section{Benchmark 2: Literature-Reported Operation}

Based on the literature $[27,28]$, what LTL companies do in practice is to solve the network design problem, find the service links and operate on those links by routing shipments over the network. The routing plan is also called load plan or service network plan, and includes a series of routing instructions of the form: shipment at terminal $i$ with destination $j$ should be assigned to truck headed to a specific terminal (regardless of the origin terminal). So, all shipments between each pair would be assigned to the same path, each time. There is no shipment split. They manage to keep a frequency of trucks running on each link to handle the shipment delivery task. 
In order to create a similar solution to what carriers do in practice the heuristic search algorithm is modified and used. The in-depth discussion on the proposed method will be provided in the next section. In real world operation, only the 1st shortest path is selected to route the shipments. The shipments are assigned to the first available outbound truck. Shipments are not held at any intermediate hubs unless there is no truck to take them to their next stop. To mimic the literature-reported operations, the empty trucks movements are also allowed.

The program uses the shipment and truck data as inputs and calculates the objective function. The program generates a set of formatted outputs that can be utilized by "operation plan" program to generate the performance measures. The results are used as a benchmark to compare with the solution provided by the proposed heuristic algorithms.

\section{Benchmark 3: Lower Bound}

The combined shipment and truck routing MIP problem is an NP-hard combinatorial optimization problem. Solving such problems using the exact solution methods (e.g. CPLEX) requires an amount of time that increases exponentially with the problem size. Therefore, the approximation algorithms are often used to find good solutions in a reasonable amount of time. The objective of the problem is to minimize the total operational costs. Hence, the value that is obtained for the lower bound can be used as a benchmark to check the quality of the solutions generated by approximation methods.

Lower bounds on the minimum solution value are used not only in evaluating the quality of approximate solutions but also in limiting the search effort to find the optimum solution. The Lagrangian relaxation is the most common method to identify the lower bound for minimization problems. However, the MIP problem that is formulated in this study is a very complex problem that contains a large number of integer variables. Hence, even the Lagrangian relaxation requires a very long computation time and this method is not implemented in this study. An easy way to 
find the lower bound is by dropping some of the constraints and solving the remaining simpler problem that can be done within a short time. The $3^{\text {rd }}$ benchmark (lower bound) is generated using this method.

Figure 60 shows the modifications that have been applied to the mathematical program to derive the lower bound on the solution value. It is assumed that unlimited number of trucks is available at each terminal. Therefore, there is no capacity on the fleet size and there will be no need to make the empty movements. This assumption is captured by removing 2 constraints from the original MIP problem; the truck conservation constraints and the integrality constraints for empty trucks.

In LTL trucking operations, the shipment size (weight) is not necessarily an integer value; therefore in the general formulation of the problem, there is no integrality constraint on shipment decision variables. However, in the more complex instance of the problem that is used for the numerical experiments the shipments are cars, and therefore the integrality constraints are included.

Auto carriers are one class of motor carriers that transport vehicles throughout the United States for individuals. Auto carriers are similar to LTL trucking companies in terms of network structure, size of shipments and type of operations. In this study, the numerical experiments are conducted for these types of motor carriers. In this type of trucking operations the shipments are cars. This important fact imposes an integrality constraint on the decision variables related to volume of shipments. To find the lower bound, in addition to the constraints related to empty trucks, all shipment related integrality constraints are also dropped.

The relaxed optimization problem is generated using a modified "Generate Problem" C program. The numerical experiments on different size of networks are performed and the results are reported in the next Chapter. The lower bound provides a tight bound on the minimum value of the objective function and is used as the benchmark to check the quality of heuristic solutions for the large size problems, where the solution to MIP problem cannot be obtained using CPLEX. 
Minimize

$\sum_{t=0}^{T}\left[\sum_{l \in L R(t)}[X F(l) \cdot C F(l)]+\sum_{l \in L R(t)}[X E(l) \cdot C E(l)]\right]+\sum_{t=0}^{T} \sum_{l \in L R(t)} \sum_{s \in S}[X S(s, l) \cdot C H(s)]+$

$\sum_{t=0}^{T} \sum_{l \in L W(t)} \sum_{s \in S}[X S(s, l) \cdot C W(s)]+\sum_{s \in S} \sum_{n \in N-\{D(s)\}}\left[\left(\sum_{l \in L E(n, T)} X S(s, l)\right) \cdot C N(s, n)\right]+$

$\sum_{s \in S}\left[\sum_{t=T L(s)+1}^{T}\left(\left[\sum_{l \in L E(D(s), t)} X S(s, l)\right] \cdot(t-T L(s)) \cdot C L(s)\right)\right]$

Subject to:

$$
\begin{array}{lc}
\sum_{l \in L B(O(s), T E(s))} X S(s, l)=W S(s) & \forall s \in S \\
\sum_{t=0}^{T} \sum_{l \in L E(O(s), t) \cap L R(t)} X S(s, l)=0 & \forall s \in S \\
\sum_{t=0}^{T} \sum_{l \in L E(D(s), t)} X S(s, l) \leq W S(s) & \forall s \in S \\
\sum_{t=0}^{T} \sum_{l \in L B(D(s), t)} X S(s, l)=0 & \forall s \in S \\
\sum_{l \in L E(n, t)} X S(s, l)=\sum_{l \in L B(n, t)} X S(s, l) & \forall s \in S, \forall t \in[0, T], \forall n \in N-\{O(s), D(s)\} \\
\sum_{s \in S} X S(s, l) \leq X F(l) . W F & \forall l \in L R(t), \forall t \in[0, T] \\
S E(n, t)+\sum_{l \in L E(n, t)}[X F(l)+X E(l)]=\sum_{l \in L B(n, t)}[X F(l)+X E(l)] & \forall n \in N, \forall t \in[0, T] \\
X S(s, l) \geq 0 & \forall s \in S, \forall l \in L R(t) \cup L W(t), \forall t \in[0, T] \\
X F(l)=0,1,2, \ldots & \forall l \in L R(t), \forall t \in[0, T]
\end{array}
$$

Figure 60 - Modified mathematical problem used to derive the lower bound 


\section{Algorithm 1: Path-Based Heuristic Search}

Unlike the exact solution methods (e.g. branch and bound), the heuristic search algorithms are designed to find sufficiently "good" solutions for large scale optimization problems. Starting with an initial feasible solution, the search algorithm iteratively performs small changes to this solution in order to move to a neighbor feasible solution with an improved value of objective function. The iterative improvements change the current solution to one in its neighborhood that has a lower cost. The algorithm stops when no better neighbor exists.

The first proposed solution algorithm is a local search procedure that is performed to find the best combination of shipment and truck paths. As discussed earlier, in order to decrease the size of the search area, instead of considering link volumes as the variable the shipment paths are used as the primary variables of the search process.

In tactical planning for LTL trucking operations all loads with the same origin and destination are routed through the same path, which is the shortest path that connects their origin and destination. This is a simplifying rule that is also used by trucking companies in their daily operation. In this study, unlike what is being done in practice and in tactical planning, shipments with the same origin and destination are allowed to be separated and routed through different paths.

Figure 61 presents an example to show how LTL trucking companies can avoid the risk of underutilizing their resources by using the multi-path approach in routing the shipments. The physical network, demand information and travel times are shown in Figure 61. The fleet is assumed to be homogeneous with the truck capacity that is equal to 10 units. Based on the assumptions, here are the best 2 shortest paths between terminal 2 and terminal 4:

First shortest path: 2-5-4

Second shortest path: 2-3-4 
If the shipment number 2 is routed using the $1^{\text {st }}$ shortest path, there will be 6 units of loads on links $2-5$ and 5-4. On the other hand, when the $2^{\text {nd }}$ shortest path is used to route this shipment, the available capacity will be used more efficiently, since there will be 10 units of loads on links 2-3 and 3-4. Moreover, the overall operational costs are reduced since a single truck is used to deliver all the shipments to their final destination. This example proves the crucial fact that using the shortest path to route all the shipments is not necessarily the best strategy to obtain the optimal solution. In this study, in all proposed algorithms the multi-path approach is used to route the shipments.

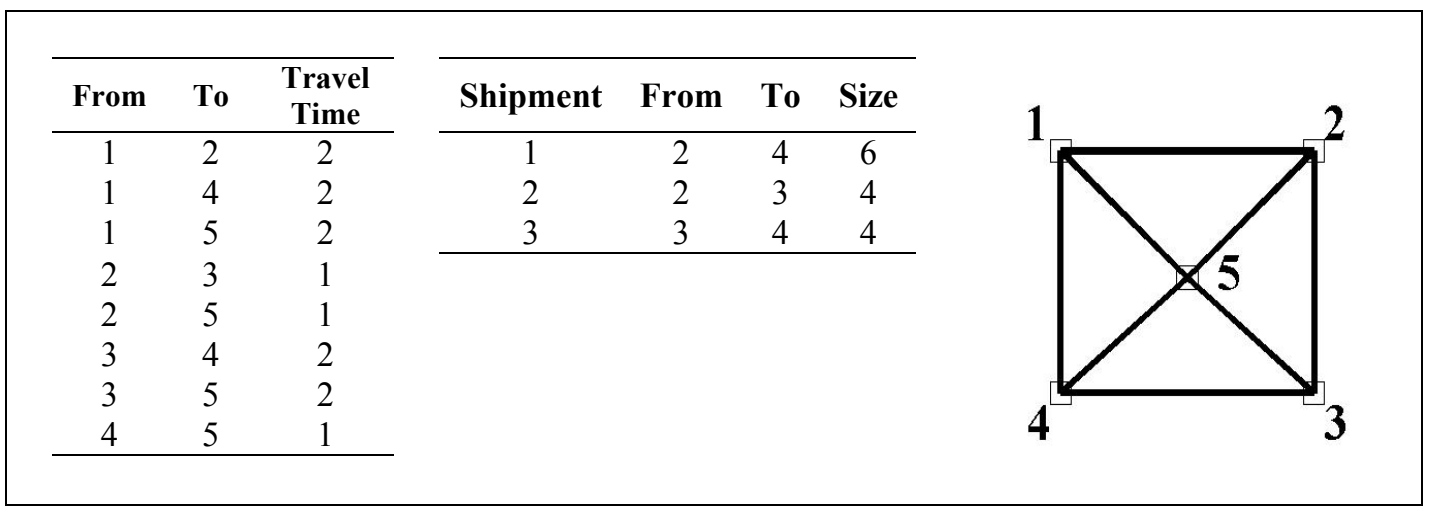

Figure 61 - Physical network (5-terminal) and demand info

A program called "Problem Solver" is coded in C programming language to implement the path-based heuristic search. LTL network structure, fleet characteristics and shipments' information are the main inputs to the program. The initial solution is generated either randomly or using the solution obtained from the $2^{\text {nd }}$ benchmark (literature-reported operation). The initial solution consists of routing paths for each and every shipment. At this point, program enters its main loop, through which it takes a series of actions repeatedly until it reaches the limit of the number of iterations. 
The Path-based heuristic search algorithm is as follows:

Step 0 Find an initial solution that consists of routing paths for all shipments.

Step 1 If the iteration count has not reached the limit; find the neighbor of the current solution by changing the routing path for a randomly selected subset of shipments. Otherwise report the best solution.

Step 2 Calculate the total number of shipments on each link and assign them to available empty trucks.

Step 3 Schedule an empty truck to handle the unassigned shipments.

Step 4 Calculate the objective function.

Step 5 If the neighbor's solution is better than current best solution; set it as the best solution and update all related variables. Otherwise, go to Step 1.

Figure 62 shows the flowchart of the program. The following sections provide detail discussions on "shipment routing procedure", "truck dispatch procedure", "objective function calculation" and "solution improvements".

- Shipment Routing Procedure

First, the neighbor solution of the current solution is constructed. To generate the neighbor, a subset of shipments is selected randomly and their routing paths are modified. "Shipment Routing Function" is in charge of this task. Figure 63 and 64 illustrate how the program works on a 5-terminal network. It is assumed that a shipment from terminal 1 to terminal 3 is among those loads that are picked randomly. In this example, the first 7 shortest paths between 1 and 3 are being considered. Let's assume the current route for this shipment is the first shortest path between 1 and 3. One of the other 6 shortest paths is selected randomly as the new current shortest path. 


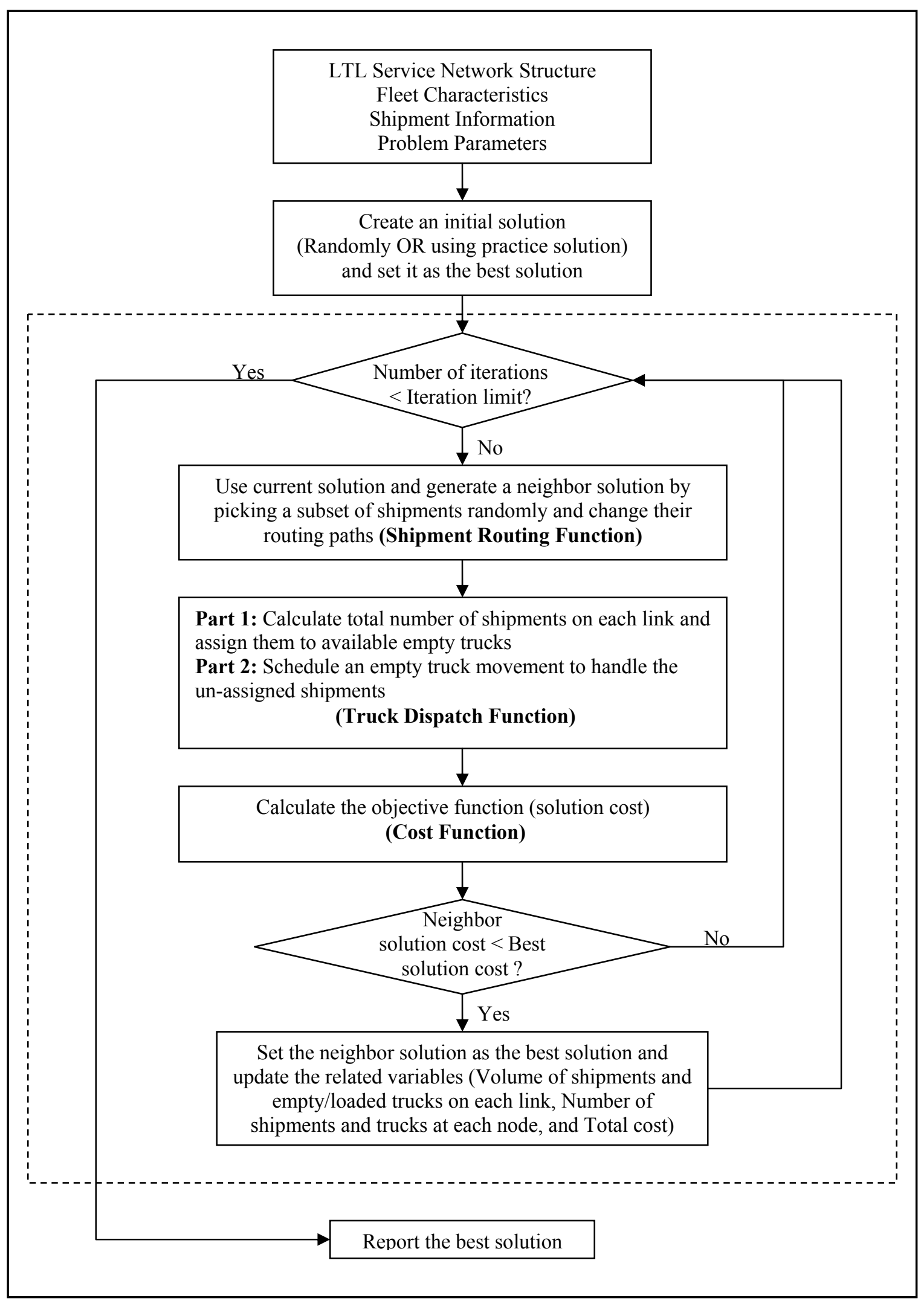

Figure 62 - Path-based heuristic search algorithm 


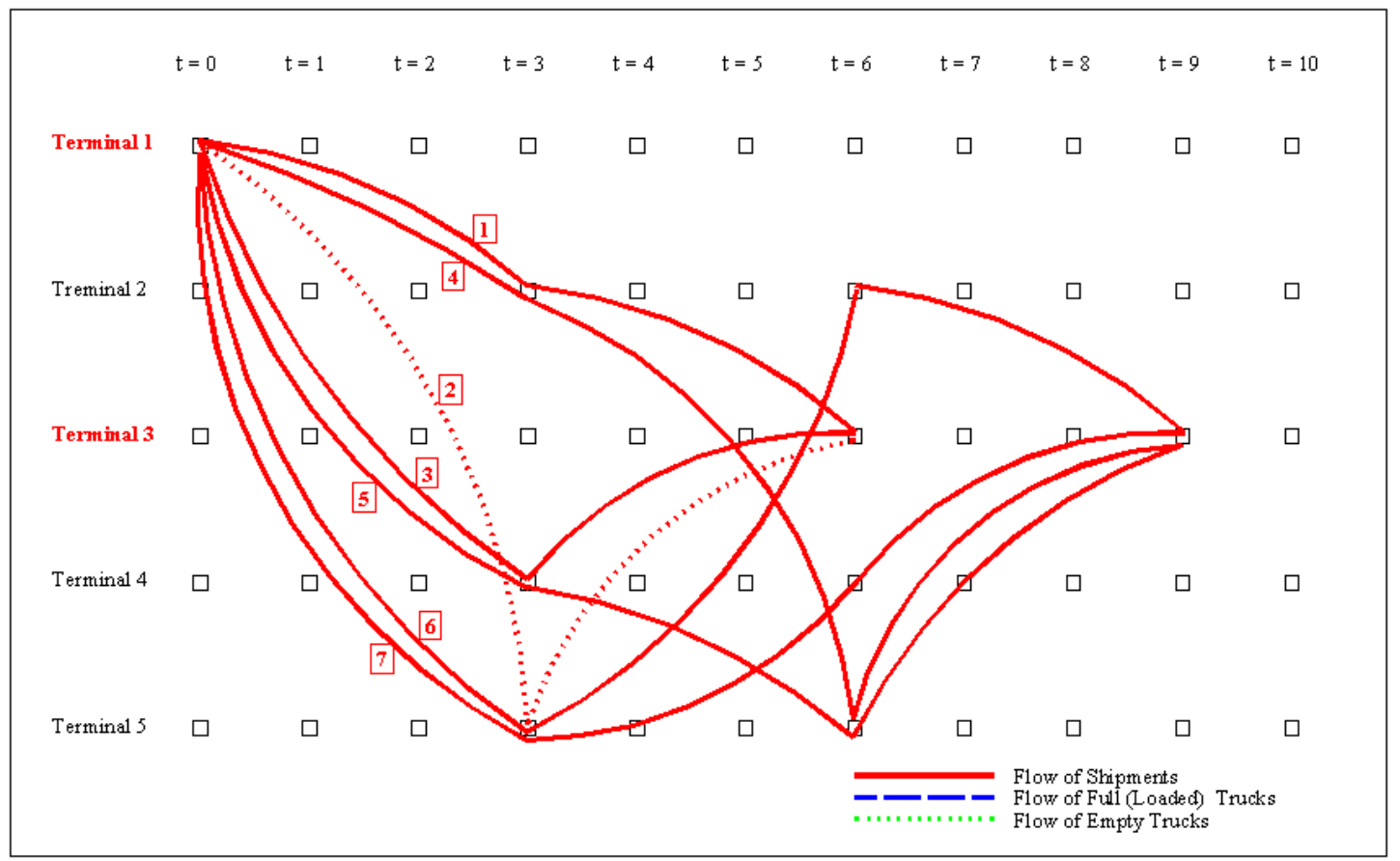

Figure 63 - Select one of the K shortest paths to route the shipment

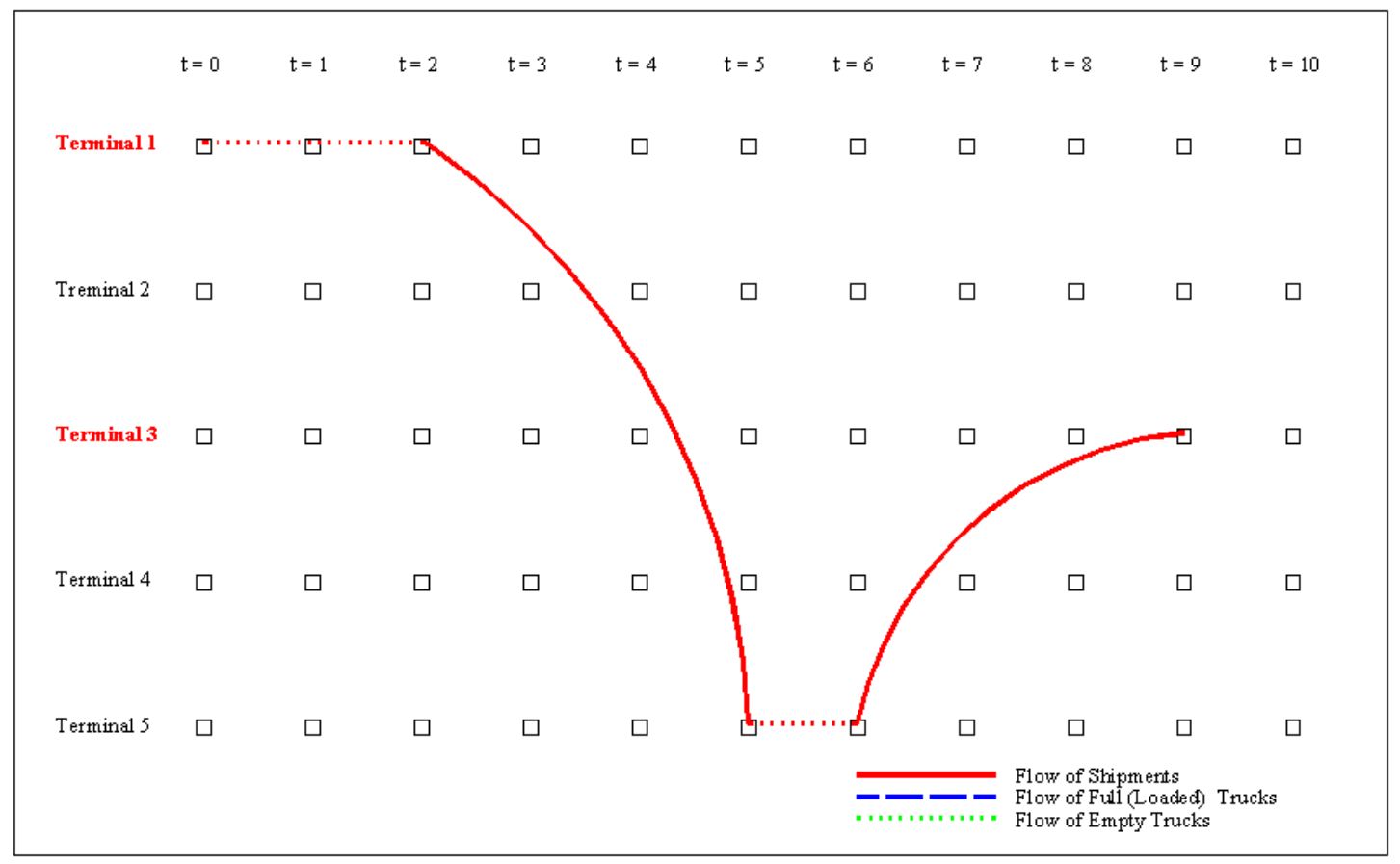

Figure 64 - Insert waiting times along the selected path to route the shipment 
The shorter shortest paths have higher possibilities to be picked as the replacement and this important fact has been incorporated into the program. After the path selection, waiting periods are inserted into the path randomly as shown in Figure 65. In this example, 2 waiting time periods added at terminal 1 (origin) and 1 waiting period added at intermediate hub, terminal 5 .

As it is shown in the above example, the path selection step is the basis of generating a new neighbor solution. Hence, a $\mathrm{K}$ shortest path routine is needed to provide the required set of potential candidates to route the shipments. Usually, the shortest path routines are called repeatedly, so the efficiency of their algorithm plays a major role in the performance of the application. In all methods that are proposed in this study, the shortest path algorithm is called only once at the beginning of the program. The all-to-all $\mathrm{K}$ shortest paths are generated and stored in arrays that are used throughout the algorithm. Therefore, the efficiency of the shortest path algorithm is not the main focus of this study since it has no impact on the overall performance of the proposed solution algorithm.

Sequential algorithms that find the generic shortest path (a single shortest path) are well known. Dijkstra [83] proposed one of the first label setting algorithms for finding the shortest path tree in a network. This algorithm is the core of the $\mathrm{K}$ shortest path algorithm that is implemented in this study.

A very large body of research has focused on single-path as well as multiplepath shortest path problems. Azevedo et al. [84],Brander et al. [85], Carraresi [86], Chong et al. [87], Consiglio et al. [88], Dryfus [89], Fox [90], Horne [91], Kumar et al. [92], Law et al. [93], Lawler [94], Martins [95], Minieka [96, 97], Perko [98], Ruppert [99], Shibuya [100], Shier [101, 102, 103], Skicism et al. [104], Weigand [105], Yen [106, 107] studied K shortest path problem and proposed algorithms and procedures to solve the problem.

The idea behind finding the K shortest path (SP) is that each two paths can share some but not all their links. The first step would be to find $1^{\text {st }}$ shortest path (SP) between every two nodes (terminals). To find the $2^{\text {nd }} \mathrm{SP}$ between each pair, all the links that appear in the $1^{\text {st }} \mathrm{SP}$ are removed, one at a time. Then, the shortest path is generated in the modified network using Dijkstra. 


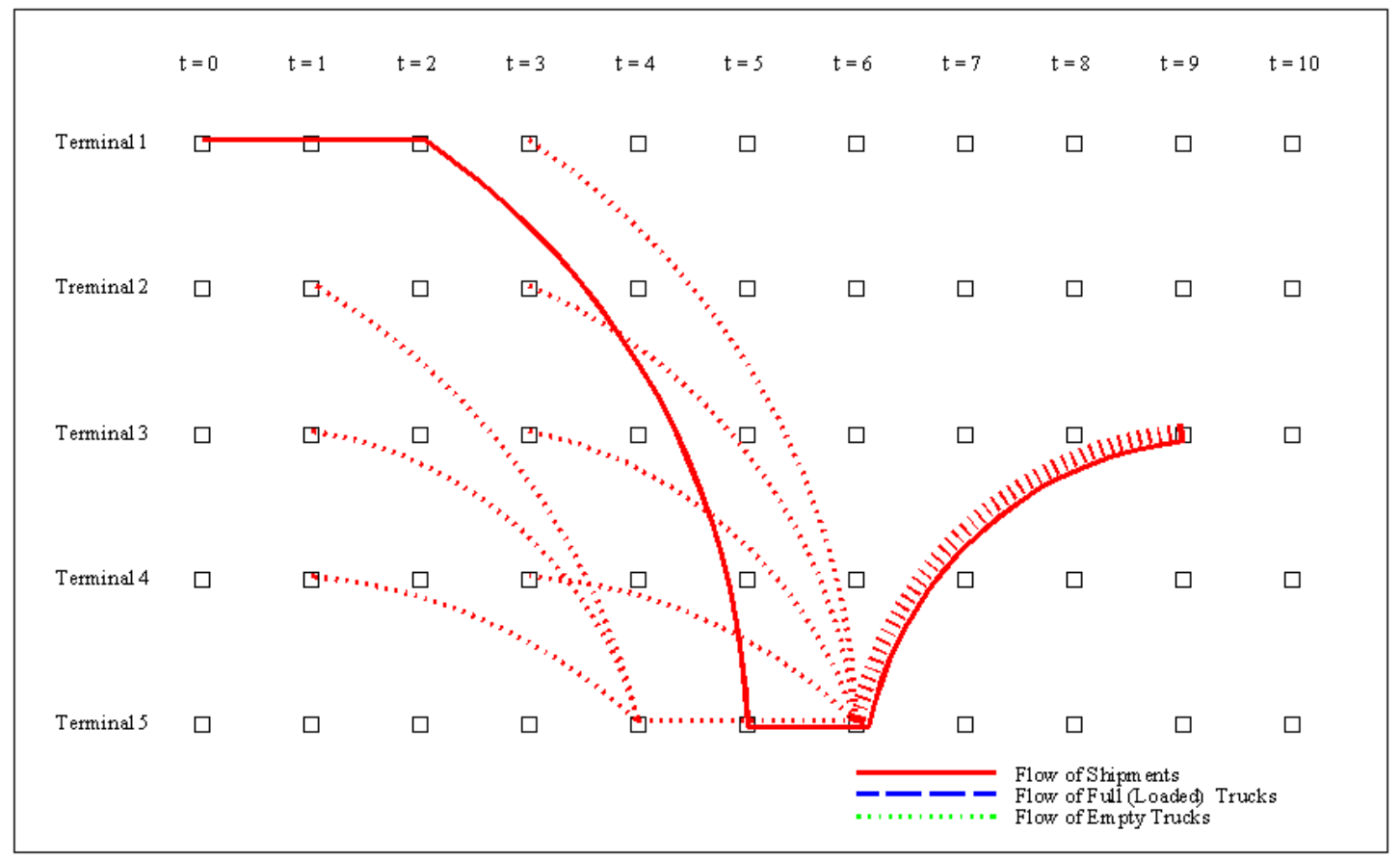

Figure 65 - Calculate the number of loads going through each link

\begin{tabular}{|c|c|c|c|c|c|c|c|c|c|c|c|}
\hline & $t=0$ & $t=1$ & $t=2$ & $t=3$ & $t=4$ & $t=5$ & $t=6$ & $t=7$ & $t=8$ & $t=9$ & $t=10$ \\
\hline Terminal 1 & $\square$ & $\square$ & $\square$ & $\square$ & $\square$ & $\square$ & $\square$ & 口 & $\square$ & $\square$ & $\square$ \\
\hline Treminal 2 & $\square$ & $\square$ & $\square$ & $\square$ & $\square$ & $\square$ & $\square$ & $\square$ & $\square$ & $\square$ & $\square$ \\
\hline Terminal 3 & $\square$ & 口 & $\square$ & $\square$ & $\square$ & $\square$ & $\square$ & $\square$ & $\square$ & إنغ & . . . \\
\hline Terminal 4 & 口 & $\square$ & $\square$ & 口 & 口, & $\square$ & $\square$ & & $\square$ & $\square$ & $\square$ \\
\hline Terminal 5 & $\square$ & $\square$ & $\square$ & $\square$ & $\square$ & $\square$ & 6 & $\square$ & $\square$ & $\square$ & $\square$ \\
\hline & & & & & & & & & \multicolumn{3}{|c|}{$\begin{array}{l}\text { Flow of Shipmentis } \\
\text { Flow of Full (Loaded) Trucks } \\
\text { Flow of Empty Trucks }\end{array}$} \\
\hline
\end{tabular}

Figure 66 - Dispatch empty/loaded trucks to handle the loads 
For example if there are 5 links on the $1^{\text {st }}$ SP by removing one link at a time, 5 candidates are generated and the shortest one would be the $2^{\text {nd }}$ SP. To find the $3^{\text {rd }}$ SP there are 2 sets of links; one for the $1^{\text {st }} \mathrm{SP}$ and the other one for the $2^{\text {nd }}$ SP. Two links are removed (one from each set) and the $3^{\text {rd }} \mathrm{SP}$ is the shortest path that is generated for the modified network.

- Truck Dispatch Procedure

The next step is performed using "Truck Dispatch Function", where the available trucks are assigned to the loads. First, the total number of shipments on each link is calculated. Then, the program starts from the first time period and moves forward. Shipments are assigned to the available empty trucks at each terminal. If needed, the program checks the possibility of empty truck movement to handle the unassigned shipments. Figure 65 shows all the shipments (including the one with newly modified path), which are going to arrive at terminal 5 at time period 6 and must be moved to terminal 3. As shown in Figure 66, loads are assigned to a truck that is moved empty from terminal 4 to terminal 5 and is ready at time period 6 to haul the loads.

\section{- Objective Function Calculation}

Now that the new solution (neighbor) is generated, the program calculates the objective function. In any search algorithm, the performance can be improved significantly by reducing the time that is required to generate and evaluate the new neighbor solution. In order to increase the efficiency in calculating the objective function, all required link variables are updated as program proceeds. Therefore the data associated with shipments, empty and loaded trucks volumes are always ready. Four individual loops calculate the elements of objective function as soon as the new neighbor solution is generated. Handling, waiting, delivery failure and late-delivery costs are added and the value objective function is sent to the main function. 
- Solution Improvements

The objective function for the neighbor solution and the current best solution are compared. If the neighbor solution is worse than the current one, program goes back to the beginning of loop and generates the next neighbor. If the solution is improved, the new solution would become the current best solution and the related variables need to be updated. The volume of shipments and empty/loaded trucks on each link, the number of shipments and trucks at each node and the total cost are the key attributes of each solution and are updated each time that a better solution is found. The algorithm terminates when the specified stopping criteria is reached. The stopping criteria are determined using a set of preliminary runs and based on the timeframe that is available to run the program. In heuristic search algorithms more iterations and longer search would lead to better results. The outputs follow the CPLEX format, so by running the "operation plan" program all the performance measures can be generated. 


\section{Algorithm 2: Path-Based Heuristic Search with Simulated Annealing}

The heuristic search algorithm that is proposed in the previous section provides a locally optimal solution, which is not necessarily a "good" solution. Such greedy search algorithm has a fatal flaw; it can easily get stuck at local optima. Therefore, a mechanism is required to help the algorithm to escape the local optima and continue the search.

Tabu search, simulated annealing and genetic algorithm are among the most distinguished meta-heuristic approaches that have been combined with the search algorithms to improve the solutions obtained by greedy heuristics. In this study, simulated annealing (SA) is used in conjunction with the path-based search algorithm, mainly due to the simplicity of its implementation and the robustness of its application to solve the mathematical problems. A brief introduction of tabu search and genetic algorithm will be followed by a detail discussion on the path-based search algorithm with simulated annealing.

\section{- Tabu Search}

When all neighbors of the current solution are examined, the best neighbor solution is selected to be used to start the next round of search. However, there is chance that the best move from the current best neighbor solution takes the algorithm back to one the already visited neighbors. To prevent this from happening, tabu search algorithm keeps a list of solutions that had been selected as the local optimum.

\section{- Genetic Algorithm}

Genetic algorithms are inspired by the evolution process of organisms. The problem solution represents an organism's genetic string. In this algorithm, the first step is to generate a set of starting solution that is called the "population". The goal is to produce better generations through either "mutation" of randomly a selected member of the population or performing a "crossover" operation using 2 randomly 
selected members of the population (parents). Using of the newly generated population, the old population, and based on a selection strategy, the "survivor" members are selected. These survivors form the new population that is used in the next round of algorithm. These operations are performed repeatedly until the convergence is achieved. The elite members of the last population would be the solution that is provided by the genetic algorithm.

- Simulated Annealing

Simulated annealing (SA) was invented before tabu search and genetic algorithms. It was gradually improved during the past 3 decades. Metropolis et al. [108] proposed an algorithm to simulate the physical cooling process. Their algorithm was the main inspiration for Kirkpatrick et al. [109] who introduced simulated annealing algorithm as a tool to solve the optimization problems. The annealing is the heat-treatment process of metals. One can obtain desirable properties of the melted metal (i.e. hardness, flexibility...) by managing the cooling process.

Similar to tabu search algorithm, simulated annealing is a local search algorithm that moves from one neighbor to another and tries to improve the current solution. Tabu search allows "uphill move" (that make the current solution worse) only if the algorithm is stuck at a local optima, but using simulated annealing the "uphill move" can be done at any time.

Simulated annealing algorithm utilizes a control parameter called "temperature" to simulate the physical annealing process. The temperature controls the possibility of moving to a worse neighborhood solution. As the search proceeds, the temperature is lowered. During high temperature phase more random movements are allowed within the solution. This would help the algorithm to escape from local optima. As algorithm gradually goes toward a good solution the temperature decreases until the algorithm reaches the minimum defined temperature and settles into so-called "frozen" state. 
The following procedure shows the outline of the SA technique that has been adapted for the minimization problem based on Kirkpatrick's algorithm [109]:

Step 0 Find an initial solution s(initial), Choose a method to find a neighborhood solution, an initial temperature t(initial), a cooling function $F(t)$, number of iteration at each temperature $N$ and the stopping condition (e.g. a final temperature $t$ (final) or total number of iteration ).

Step 1 Find $s$ (neighbor) the neighbor of the current solution $s$ (current).

Step 2 Calculate $\Delta$ which is the difference in their objective functions $\Delta=Z($ s(neighbor $))-Z(s$ (current) $)$

Step 3 Decide whether to accept the new solution or not: If $s$ (neighbor) is better than $s$ (current), set s(neighbor) as $s$ (current). If $s$ (neighbor) is worse than $s$ but passes the Boltzmann trial $\exp (-\Delta / T)>$ random $U(0,1)$, set $s$ (neighbor) as $s$ (current).

Step 4 If $s$ (neighbor) is better than current best solution $s$ (best), set it as the best solution.

Step 5 If the iteration count at the $t$ (current) reaches $N$, reduce the temperature using $F(t)$.

Step 6 If the stopping conditions are satisfied (e.g. t(current) is less than $t$ (final) or the total iteration limit has reached), output the $s$ (best) as the final solution. Otherwise, start from step 1.

To implement this general simulated algorithm one needs to identify the elements of the cooling process, which includes: the initial temperature, the final temperature, the cooling function and the number of iterations at each temperature.

Generally, the initial temperature is selected high enough to allow uphill moves during the early stages of the process. However, when the SA algorithm starts with a high quality initial solution, it is suggested to use a lower temperature. Kirkpatrick et al. [109], Dowsland [110, 111], Johnson et al. [112, 113] and BenAmeur [114] have suggested different methods to determine the initial temperature. 
The most popular cooling function is the linear function $(f(t)=\alpha t)$ that is also used in this study. The temperature reduction rate is a constant in range of $[0.80$, 0.99]. In very low temperatures, most of the solutions are rejected. This might have a negative impact on the performance of the algorithm. Hence, some researchers suggest a reheating process when algorithm is stuck in local optima at a very low temperature.

Different stopping conditions are suggested in the literature. The conventional method is to stop the algorithm when it reaches the frozen state. The other idea is to stop the algorithm when the acceptance ratio of the uphill moves is less than a predefined minimum. The computation time and the total number of iterations are among the other parameters that can be monitored to determine a stopping point for the algorithm.

As discussed previously, the heuristic search algorithm that is proposed produces a locally optimal solution, which is not necessarily a "good" solution. When the algorithm gets stuck in local optima, a mechanism is required to make the search process escape from the local optima. One alternative, which is used in this study, is to apply meta-heuristic methods such as simulated annealing (SA) combined with the path-based search algorithm.

The Path-based heuristic search algorithm with simulated annealing is as follows:

Step 0 Find an initial solution that consists of routing paths for all shipments and set it as current solution.

Step 1 If the iteration count has not reached the limit; find the neighbor of the current solution by changing the routing path for a randomly selected subset of shipments. Otherwise report the best solution.

Step 2 Calculate the total number of shipments on each link and assign them to available empty trucks.

Step 3 Schedule an empty truck to handle the unassigned shipments. 
Step 4 Calculate the objective function.

Step 5 If neighbor's solution is better than current best solution; set it as the current and the best solution and start from Step 1.

Step 6 If the neighbor's solution is better than current solution OR if it is worse than current solution but it passes the Boltzmann trial; set it as the current solution. Go to Step 1.

Figure 67 shows how the simulated annealing procedure is incorporated into the heuristic search algorithm. The flowchart introduces the second algorithm that is proposed in this study. The next Chapter provides the detail discussion on the implementation of this algorithm to solve the shipment and truck routing MIP problem. 


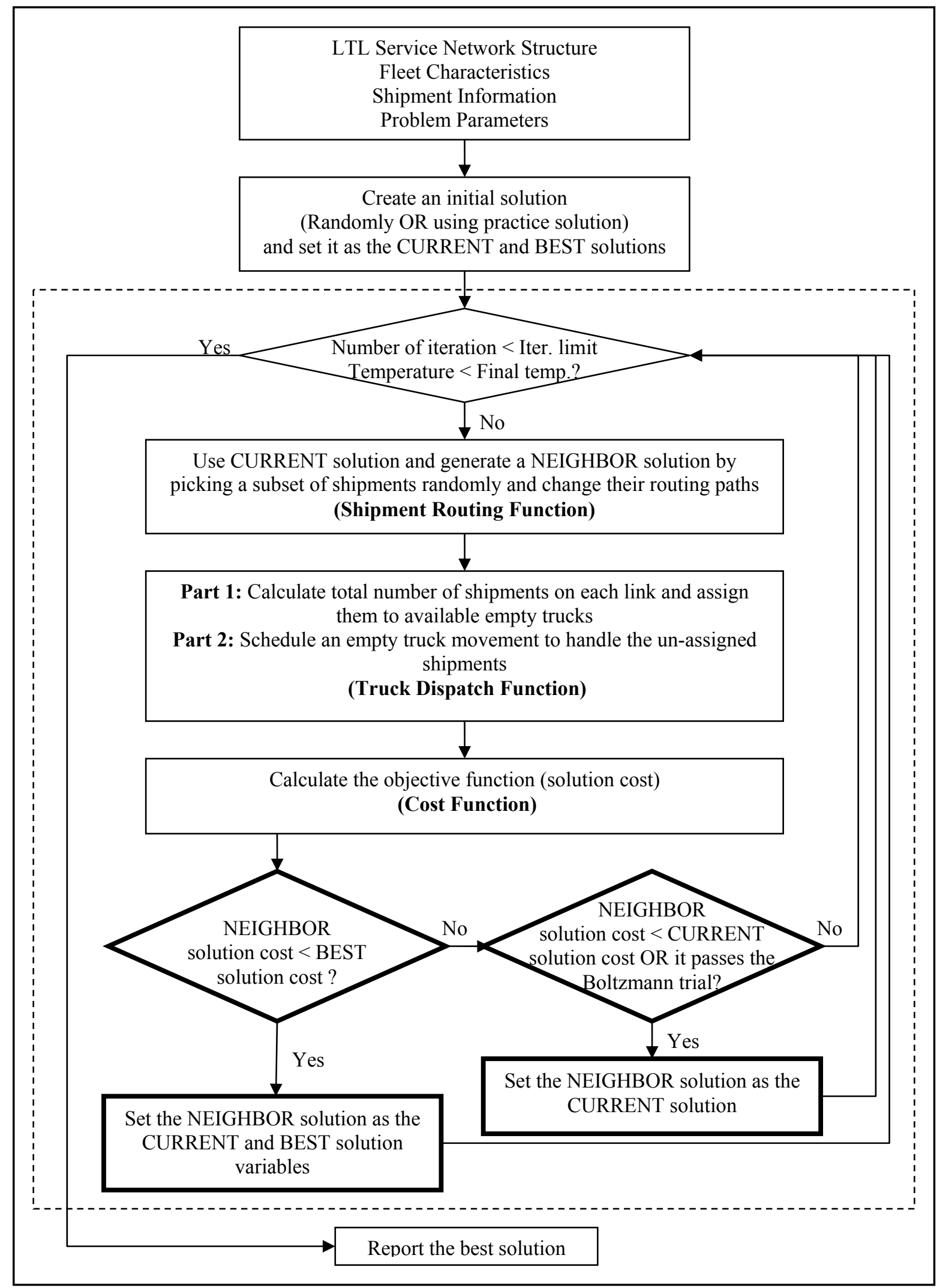

Figure 67 - Path-based heuristic search with simulated annealing 


\section{Algorithm 3: Path-Based Network Partitioning}

The heuristic search with and without simulated annealing is used to solve the mathematical problem. The results of the numerical experiments on different size networks are reported in the next Chapter. The proposed heuristic search can improve the solution quality compared to what is being done in practice. However, even for the smaller size problems the results are far from the optimal solution provided by CPLEX. In an effort to further improve the quality of the solution, the third proposed solution algorithm seeks to take advantage of path-based network partitioning.

The path-based network partitioning that is presented in this section is inspired by the procedure that is used to find the neighbor solution in the previous solution algorithms. Routing costs play a major role in total operational costs of the carrier. The carrier may reduce the overall routing costs simply by routing the shipments through the shortest path. However, as it was proven in the last section, this method is not necessarily the best way to obtain the optimal solution. An alternative would be to generate and pick the shipment routing path using the first Kshortest path. This method takes advantage of consolidation opportunities to avoid underutilizing the system's resources.

As the $3^{\text {rd }}$ proposed method to solve the mathematical problem, a path-based network partitioning is used in conjunction with an exact solution algorithm (e.g. branch-and-bound). When the problem is solved using CPLEX, all possible paths to route a shipment are checked implicitly. The optimal solution presents the best set of the routing paths after considering all different combinations. However, in majority of cases the path that is picked by CPLEX to route the shipment is among the first $\mathrm{K}$ (e.g. 4 or 5) best shortest paths. The idea is to reduce the network size for each shipment by choosing only the links that appear in the first $\mathrm{K}$ shortest path for that particular shipment.

Partitioning the network reduces the number of decision variables significantly. Hence, it would be practical to use the exact solution methods to solve the resulting modified MIP problem for large size networks.

In this section, the path-based network partitioning method is described using the medium size network with 10 terminals. This is the same network that is also used 
to perform the numerical experiments (Figure 68). All the links are assumed to be 600 miles long and undirected. Table 19 lists the characteristics of the first 8 shortest paths between New York and Kansas City (Terminal 4 to Terminal 5).

The maximum reduction in network size is obtained by using only one path to partition the network. As shown in Figure 69, for shipment from 4 to 5 the reduced network consists of 3 links; 4-3, 3-2 and 2-5. Before solving the problem using CPLEX, the volume of the shipments from 4 to 5 is pre-set to zero for all links other than the above 3 links. Assuming that there are 10 time periods in the time-space network for this problem, the number of pre-set decision variables for this particular shipment would be over 300. When only the first shortest path is used for partitioning, the resulting network is called "SP-1".

SP-2, SP-3 and SP-8 are presented in Figure 70 to 72 . Some of the links are shared among the shortest paths. The number of links in SP-2, SP-3 and SP-8 modified networks is 5,8 and 14 , respectively. Naturally, considering more paths in the modified network increases the computation time. However, the optimal solution will be closer to that of the original non-modified problem. Therefore, a set of preliminary numerical experiment is required to find the optimum number of shortest path to be used in the network partitioning process. Figure 73 illustrates the result multi-path routing network considering the reduced size network for all the shipments.

To construct the SP-K network the shortest paths are selected based on their ranks for each O-D pair. Tables 20 and 21 list the length of 8 first shortest paths from terminal 1 and 5 to all other terminals in a 17-terminal network. This large size network is also used for the numerical experiments presented in the next Chapter. The length of the shortest path for each pair does not increase linearly. Therefore, when the paths are chosen based on their ranks, it is quite possible that some noncompetitive paths are selected while some of the competitive paths are ignored. Hence, choosing the set of paths based on their rank is not necessarily the best strategy. A variation to this method is to use the length of the path as the selection criteria. 


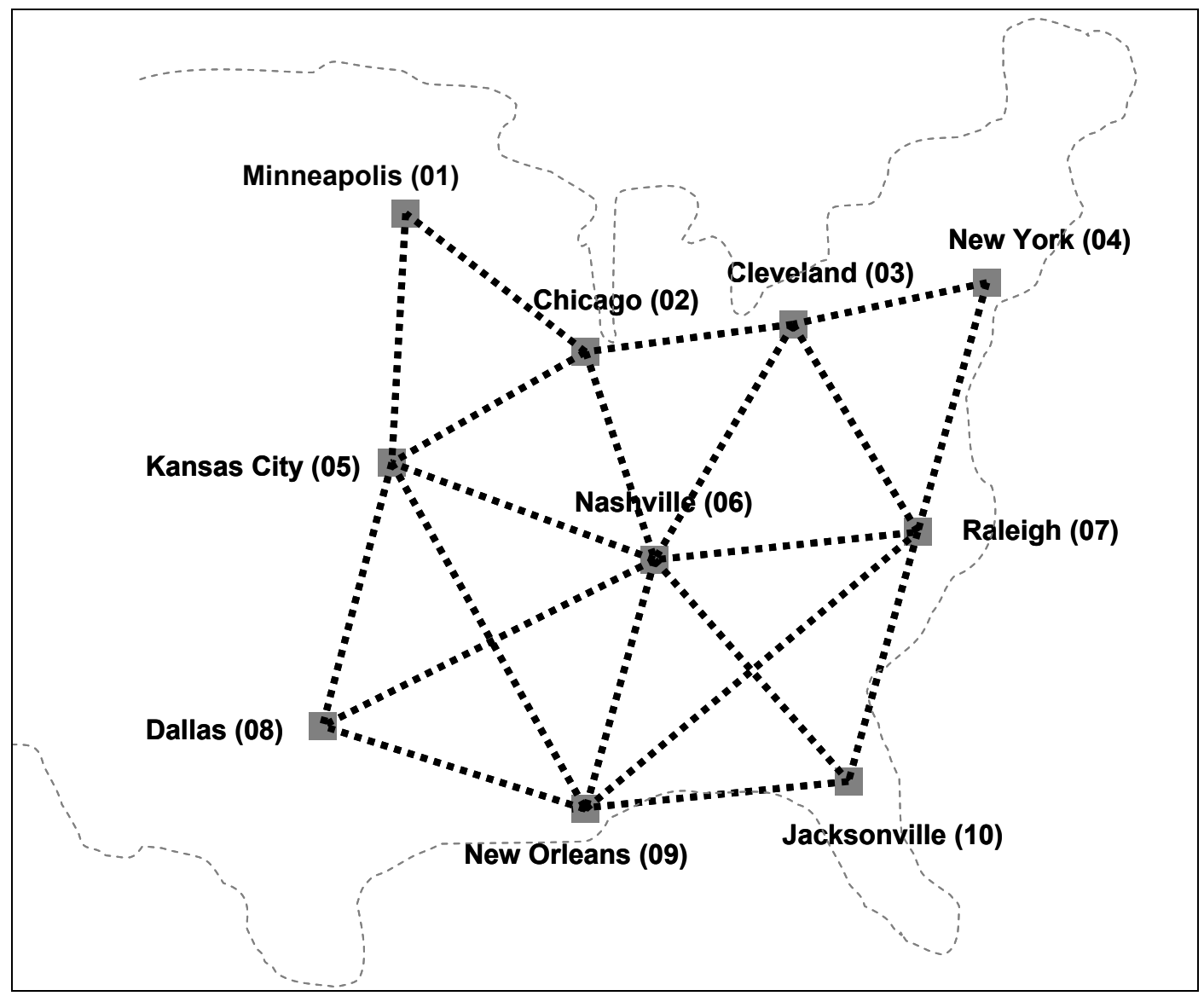

Figure 68 - Physical network (10-terminal)

Table 19 - Characteristics of the first 8 shortest paths from terminal 4 to terminal 5

\begin{tabular}{|c|c|c|c|c|c|c|c|c|c|}
\hline \multirow{2}{*}{$\begin{array}{l}\text { Shortest Path } \\
\text { 1st Shortest Path }\end{array}$} & \multicolumn{7}{|c|}{ Path } & & \multirow{2}{*}{$\begin{array}{c}\begin{array}{c}\text { Approximate } \\
\text { Length (miles) }\end{array} \\
1800\end{array}$} \\
\hline & 4 & - & 3 & - & 2 & - & 5 & & \\
\hline 2nd Shortest Path & 4 & - & 3 & - & 6 & - & 5 & & 1800 \\
\hline 3rd Shortest Path & 4 & - & 7 & - & 9 & - & 5 & & 1800 \\
\hline 4th Shortest Path & 4 & - & 7 & - & 6 & - & 5 & & 1800 \\
\hline 5th Shortest Path & 4 & - & 3 & - & 2 & - & 1 & - & 2400 \\
\hline 6th Shortest Path & 4 & - & 3 & - & 6 & - & 8 & - & 2400 \\
\hline 7th Shortest Path & 4 & - & 7 & - & 9 & - & 8 & -5 & 2400 \\
\hline 8th Shortest Path & 4 & - & 7 & - & 6 & - & 8 & -5 & 2400 \\
\hline
\end{tabular}




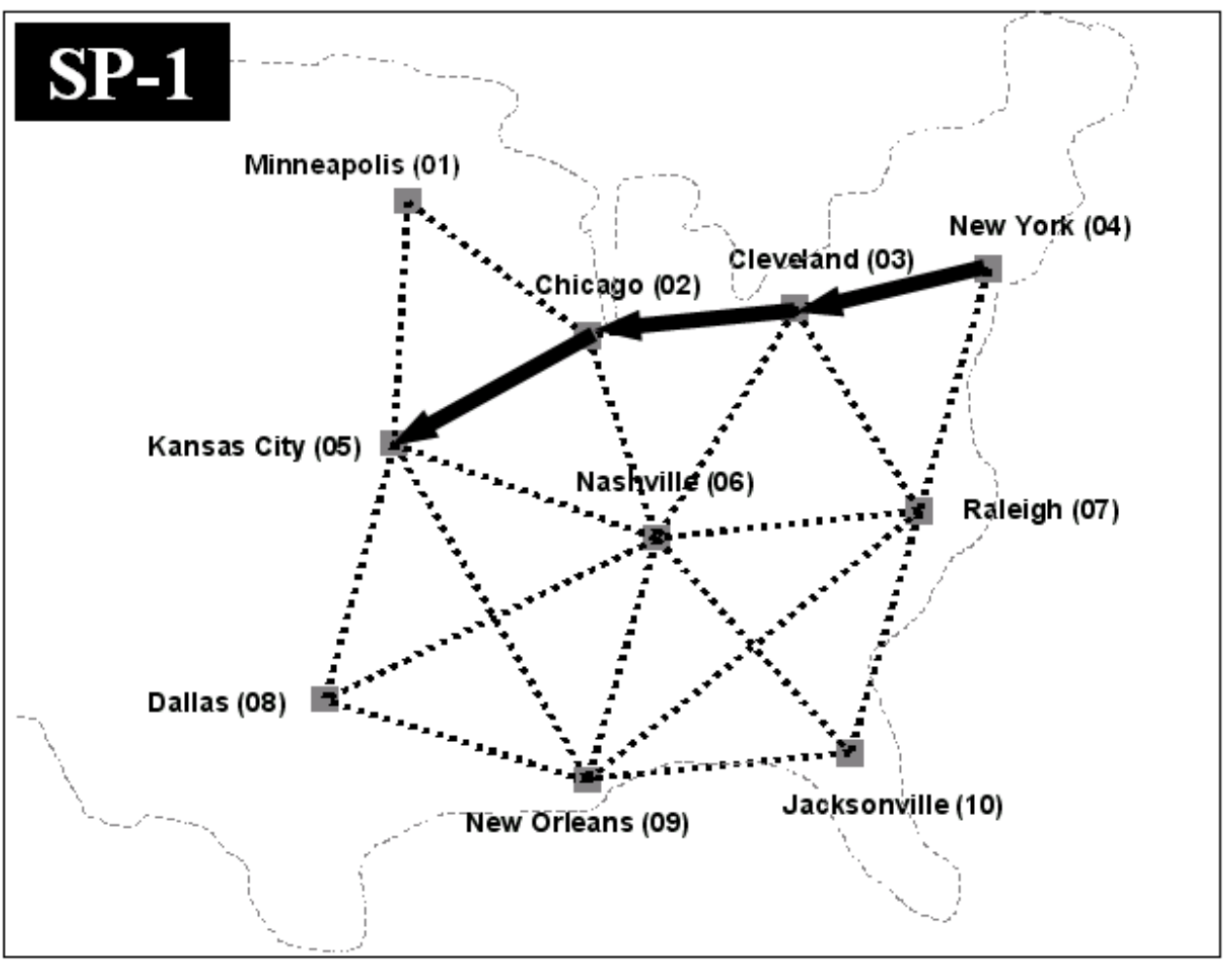

Figure 69 - Reduced network with the first SP between 4 and 5

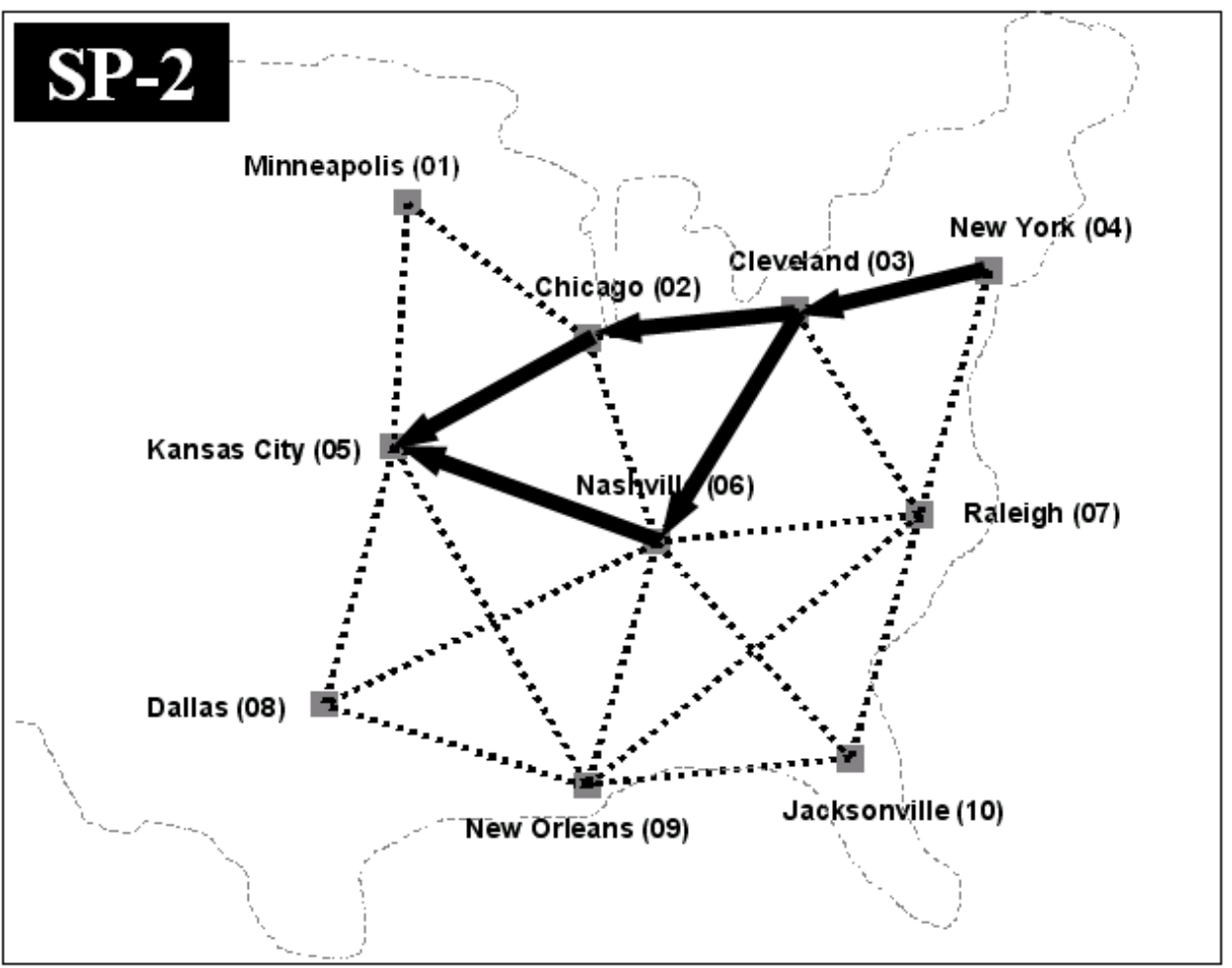

Figure 70 - Reduced network with the first two SP between 4 and 5 


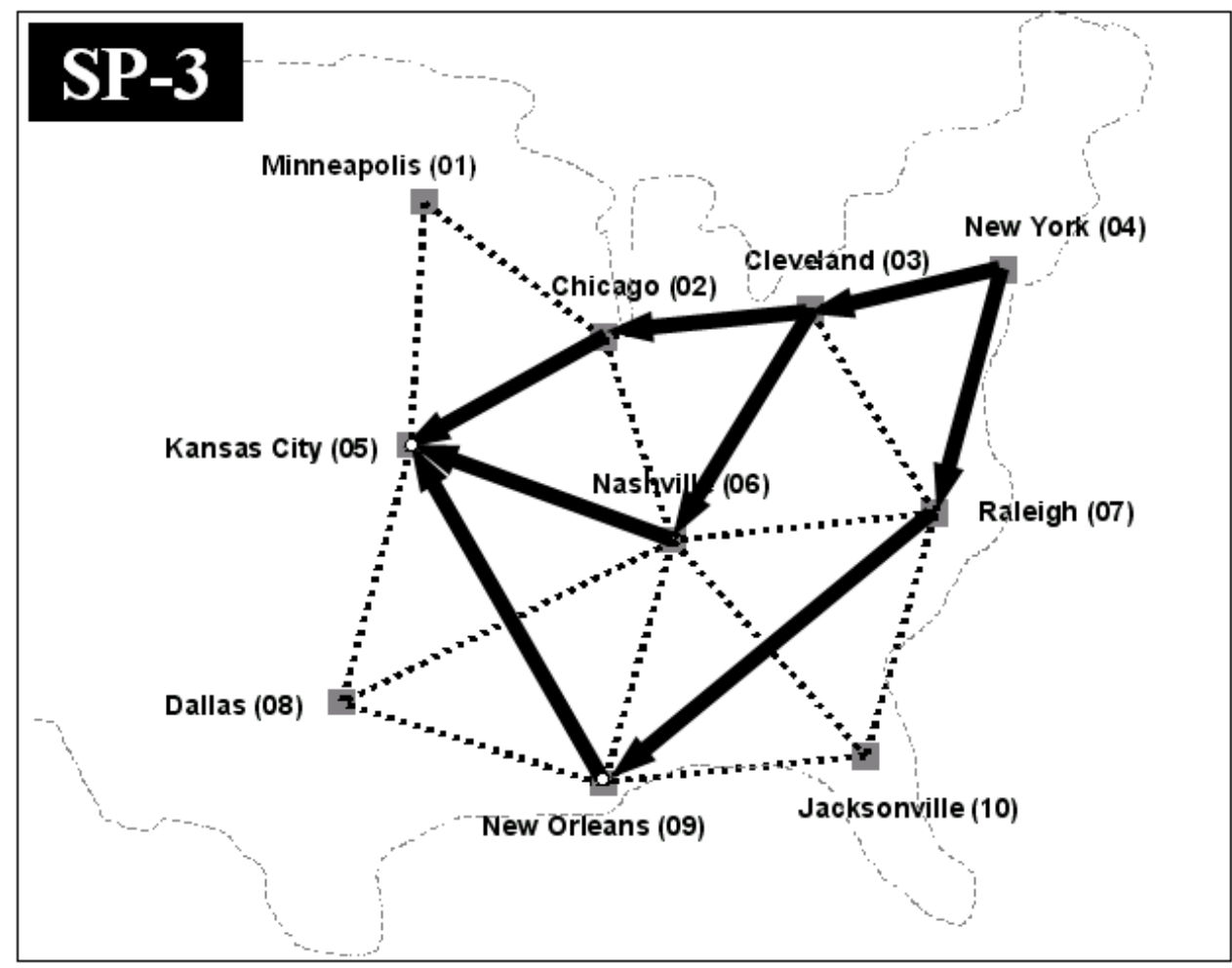

Figure 71 - Reduced network with the first three SP between 4 and 5

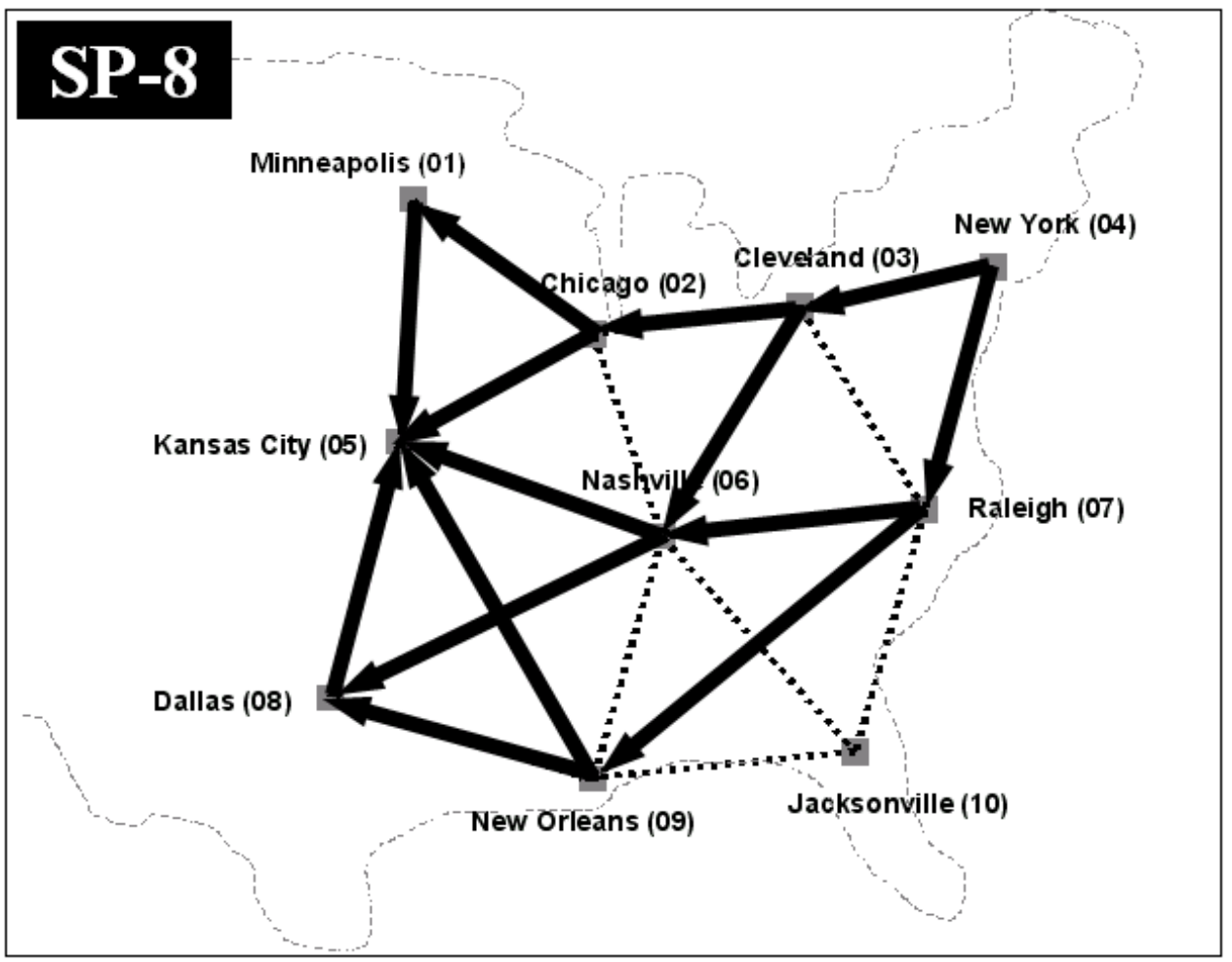

Figure 72 - Reduced network with the first eight SP between 4 and 5 


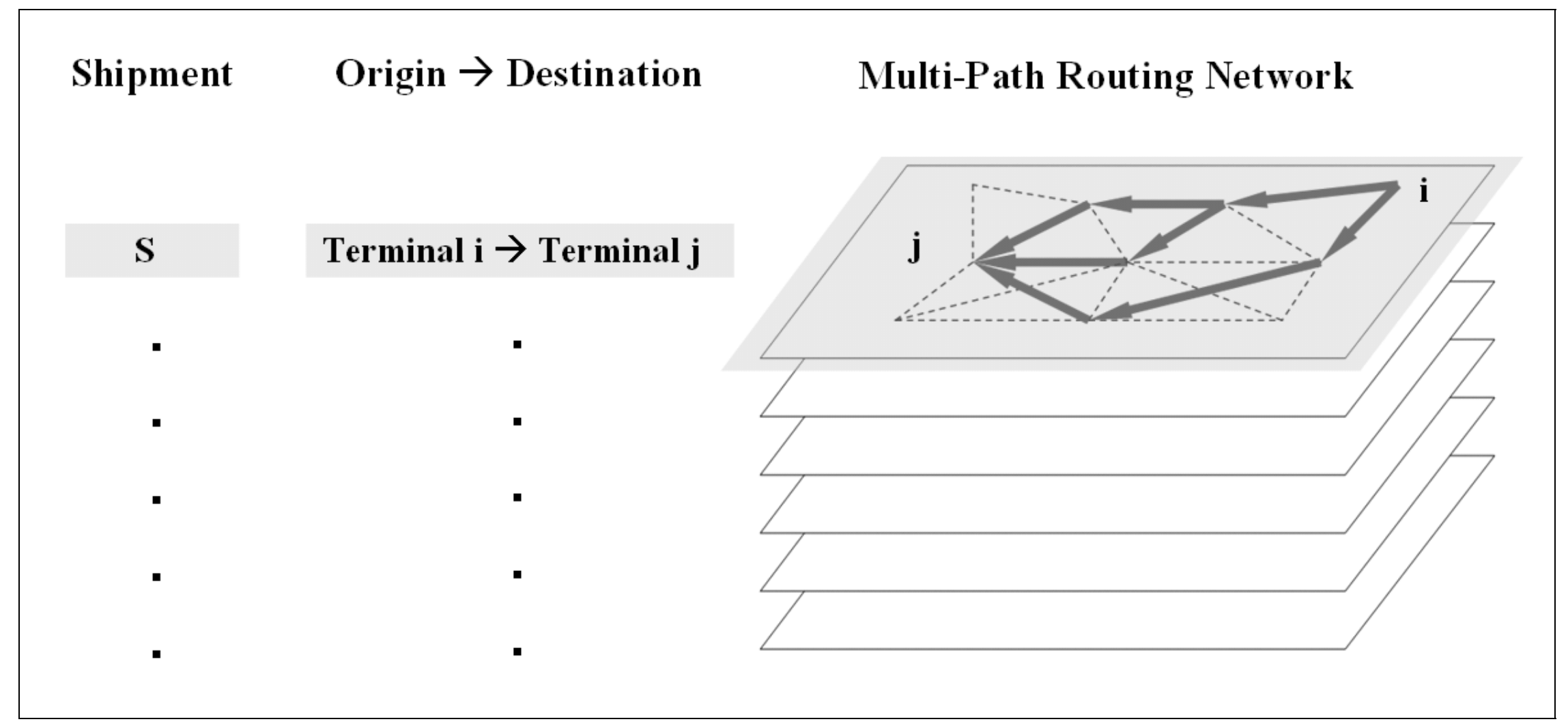

Figure 73 - Multi-Path routing network 
Table 20 - The length of the first 8 shortest paths between terminal 1 and all other terminals in the network

\begin{tabular}{|c|c|c|c|c|c|c|c|c|c|c|c|c|c|c|c|c|c|}
\hline Origin $>$ Destination & $1>1$ & $1>2$ & $1>3$ & $1>4$ & $1>5$ & $1>6$ & $1>7$ & $1>8$ & $1>9$ & $1>10$ & $1>11$ & $1>12$ & $1>13$ & $1>14$ & $1>15$ & $1>16$ & $1>17$ \\
\hline 1st Shortest Path & & 600 & 1200 & 600 & 600 & 1200 & 1800 & 1800 & 2400 & 1200 & 1800 & 2400 & 2400 & 2400 & 2400 & 3000 & 3000 \\
\hline 2nd Shortest Path & & 1200 & 1800 & 1200 & 1200 & 1200 & 1800 & 2400 & 3000 & 1200 & 1800 & 2400 & 2400 & 3000 & 2400 & 3000 & 3000 \\
\hline 3rd Shortest Path & & 1800 & 2400 & 1800 & 1200 & 1800 & 1800 & 2400 & 3000 & 1800 & 1800 & 2400 & 2400 & 3000 & 2400 & 3000 & 3000 \\
\hline 4th Shortest Path & & 1800 & 2400 & 1800 & & 1800 & 2400 & & 3000 & 1800 & & & 2400 & & & & 3000 \\
\hline 5th Shortest Path & & 2400 & 2400 & 2400 & 1800 & 1800 & 2400 & 2400 & 3000 & 1800 & 2400 & 2400 & 3000 & 3000 & 3000 & 3000 & 3000 \\
\hline 6th Shortest Path & & 2400 & 2400 & 2400 & 3000 & 2400 & 2400 & 3000 & 3000 & 2400 & 2400 & 2400 & 3000 & 3000 & 3000 & 3000 & 3600 \\
\hline 7th Shortest Path & & 3000 & 3000 & 3000 & 3000 & 2400 & 2400 & 3000 & 3000 & 2400 & 2400 & 2400 & 3000 & 3000 & 3000 & 3000 & 3600 \\
\hline 8th Shortest Path & & 3000 & 3000 & 3000 & 3000 & 2400 & 2400 & 3000 & 3000 & 2400 & 2400 & 2400 & 3000 & 3000 & 3000 & 3000 & 3600 \\
\hline Average Length & & 2025 & 2325 & 2025 & 1950 & 1875 & 2175 & 2550 & 2925 & 1875 & 2100 & 2400 & 2700 & 2925 & 2700 & 3000 & 3225 \\
\hline
\end{tabular}

Table 21 - The length of the first 8 shortest paths between terminal 5 and all other terminals in the network

\begin{tabular}{|c|c|c|c|c|c|c|c|c|c|c|c|c|c|c|c|c|c|}
\hline Origin $>$ Destination & $5>1$ & $5>2$ & $5>3$ & $5>4$ & $5>5$ & $5>6$ & $5>7$ & $5>8$ & $5>9$ & $5>10$ & $5>11$ & $5>12$ & $5>13$ & $5>14$ & $5>15$ & $5>16$ & $5>17$ \\
\hline 1st Shortest Path & 600 & 600 & 1200 & 600 & & 600 & 1200 & 1800 & 2400 & 600 & 1200 & 1800 & 1800 & 2400 & 1800 & 2400 & 2400 \\
\hline 2nd Shortest Path & 1200 & 1200 & 1800 & 1200 & & 1200 & 1800 & 1800 & 2400 & 1200 & 1200 & 1800 & 2400 & 2400 & 1800 & 2400 & 3000 \\
\hline 3rd Shortest Path & 1200 & 1200 & 1800 & 1200 & & 1800 & 1800 & 2400 & 2400 & 1800 & 1800 & 1800 & 2400 & 2400 & 2400 & 2400 & 3000 \\
\hline 4th Shortest Path & 1800 & 1800 & 1800 & 1800 & & 1800 & 2400 & 2400 & 3000 & 1800 & 1800 & 2400 & 2400 & 3000 & 2400 & 2400 & 3000 \\
\hline 5th Shortest Path & 1800 & 2400 & 2400 & 2400 & & 2400 & 2400 & 2400 & 3000 & 2400 & 2400 & 2400 & 2400 & 3000 & 2400 & 2400 & 3000 \\
\hline 6th Shortest Path & 3000 & 2400 & 2400 & 2400 & & 2400 & 2400 & 2400 & 3000 & 2400 & 2400 & 2400 & 2400 & 3000 & 2400 & 2400 & 3000 \\
\hline 7th Shortest Path & 3000 & 2400 & 2400 & 3000 & & 2400 & 2400 & 2400 & 3000 & 3000 & 2400 & 2400 & 2400 & 3000 & 2400 & 3000 & 3000 \\
\hline 8th Shortest Path & 3000 & 3000 & 3000 & 3600 & & 3000 & 2400 & 2400 & 3000 & 3000 & 3000 & 2400 & 2400 & 3000 & 3000 & 3000 & 3000 \\
\hline Average Length & 1950 & 1875 & 2100 & 2025 & & 1950 & 2100 & 2250 & 2775 & 2025 & 2025 & 2175 & 2325 & 2775 & 2325 & 2550 & 2925 \\
\hline
\end{tabular}


The highlighted paths in Tables 20 and 21 are longer than the average length of the first 8 shortest paths for each OD pair. As an alternative one would select this set of paths to partition the network. The resulting network is called SP- $8^{*}$. As seen in Tables 20 and 21, the number of paths that are selected for different OD pairs depends on the average length of the path that connects each pair. For some OD pairs all 8 shortest paths stay in the network while for some others only one shortest path participates. More discussion on comparing the different methods of network partitioning is addressed in the next Chapter.

Figure 74 shows how the proposed path-based network partitioning is embedded in the decision making procedure. The system real-time information is available either through the simulation or the real data from the field. At the end of the time horizon or each time a request arrives, the mathematical formulation of the problem is generated considering the updated empty truck locations and shipments information. A modified version of "Problem Generator" C program is developed to generate the mathematical formulation. A K-shortest path algorithm is added to the program to generate the all-to-all first $\mathrm{K}$ shortest paths. The proposed network partitioning is performed by selecting a subset of these K-shortest paths. The volume of each shipment is set equal to zero on all links except those that appear in the selected subset. The result MIP problem is solved using CPLEX. After solving the problem, the performance of the new solution is analyzed and the effects of accepting the new requests are examined based on the acceptance criteria.

One major step in all decision making procedures is to solve the shipment and truck routing problem repeatedly. In this Chapter, three heuristic solution algorithms are proposed to solve the MIP problem. The first 2 approaches are based on a search algorithm, which tries to find the best paths to route the shipments. The last approach uses a graphic partitioning to reduce the size of the problem, which is solved using CPLEX. In order to evaluate the effectiveness of the solution algorithms, three benchmark solutions are proposed; "do-nothing", "literature-reported operation" and the "lower bound". The performance of the proposed solution methods are analyzed in the next Chapter. 


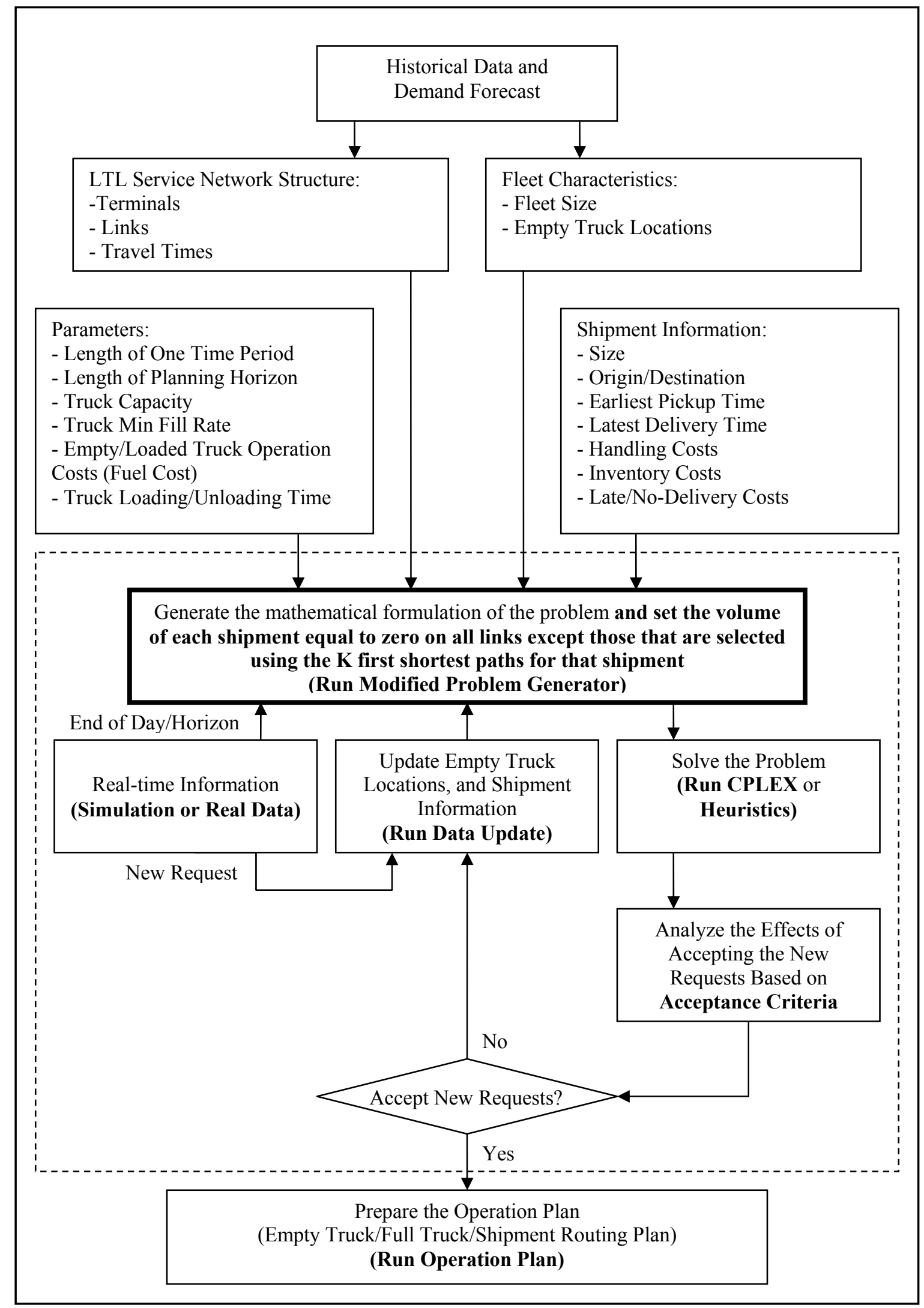

Figure 74 - Decision making procedure (with path-based network partitioning) 


\section{Chapter 7: Numerical Experiments}

The results of two numerical experiments are reported in this Chapter. The numerical experiments are conducted to analyze the performance of the proposed algorithm to solve the MIP problem. Besides, a simulation framework is introduced and the effectiveness of the proposed decision making procedure is evaluated.

As discussed in Chapter 5, Auto carriers are one class of motor carriers that transport vehicles throughout the United States. These carriers are similar to LTL trucking companies in terms of network structure, size of shipments and type of operations, so this type of carriers are selected to perform the numerical experiments in this study.

\section{Network with 10 Breakbulks}

This section presents the numerical experiment that is conducted in order to check the quality of solution provided by the proposed algorithm to solve the MIP problem. The 10-terminal medium-size network was introduced in Chapter 5 (Figure 27). The network consists of 20 undirected links that connect breakbulk terminals that are located in the proximity of 10 major cities in the US. All links are assumed to be approximately 600 miles long. Based on the preliminary numerical experiments it is assumed that the total number of time periods is 9 and the fleet size is 10 . The problem characteristics have been addressed in detail in Chapter 5 (Figure 28).

One major step in decision making procedure is to solve the shipment and truck routing problem repeatedly. For large size problem using the exact solution methods (i.e. CPLEX) to solve the MIP is not practical. Therefore, three different solution algorithms were proposed in the previous Chapter to solve the MIP problem. The first 2 approaches are based on a search algorithm, which tries to find the best paths to route the shipments. The last approach uses a graphic partitioning to reduce the size of the problem which is solved using CPLEX.

In order to evaluate the effectiveness of the solution algorithms, three benchmark solutions were also proposed; "do-nothing", "literature-reported 
operation" as upper bounds and also a "lower bound" that is derived using a relaxation method. In this section the performance of the proposed methods are analyzed using the 10-terminal network.

Figure 75 presents the objective function obtained using 4 different combination of heuristic search algorithm to solve the base case (40 shipment and 8 trucks). Here are 4 versions of heuristic search algorithm that have been analyzed:

- Heuristic search + Simulated Annealing + Literature-reported operation solution as the initial solution (Blue)

- Heuristic search + Simulated Annealing + Random initial solution (Green)

- Heuristic search + Literature-reported operation solution as the initial solution (Orange)

- Heuristic search (Red)

The optimal, do-nothing and literature-reported operation (practice) solutions are also shown in the diagram as the benchmarks. To implement the SA algorithm in conjunction with the proposed search algorithm a set of preliminary experiments were conducted to calibrate the parameters of annealing process. Instead of considering the absolute value of change in objective function, the rational value is used in the implementation of simulated annealing for this problem. Therefore, the difference in objective functions is calculated as follows:

$$
\Delta=[Z(\text { s(neighbor }))-Z(\text { s(current }))] / Z(\text { s(current }))
$$

The initial temperature is 0.05 , and the annealing process stops when the temperature reaches 0.001 . The cooling rate is 0.99 and 100 iterations are performed at each temperature. Based on the results, the heuristic search algorithm with the simulated annealing (Blue and Green) outperforms other approaches. Unlike the greedy heuristic search, the SA helps the solution to escape from the local optima. 


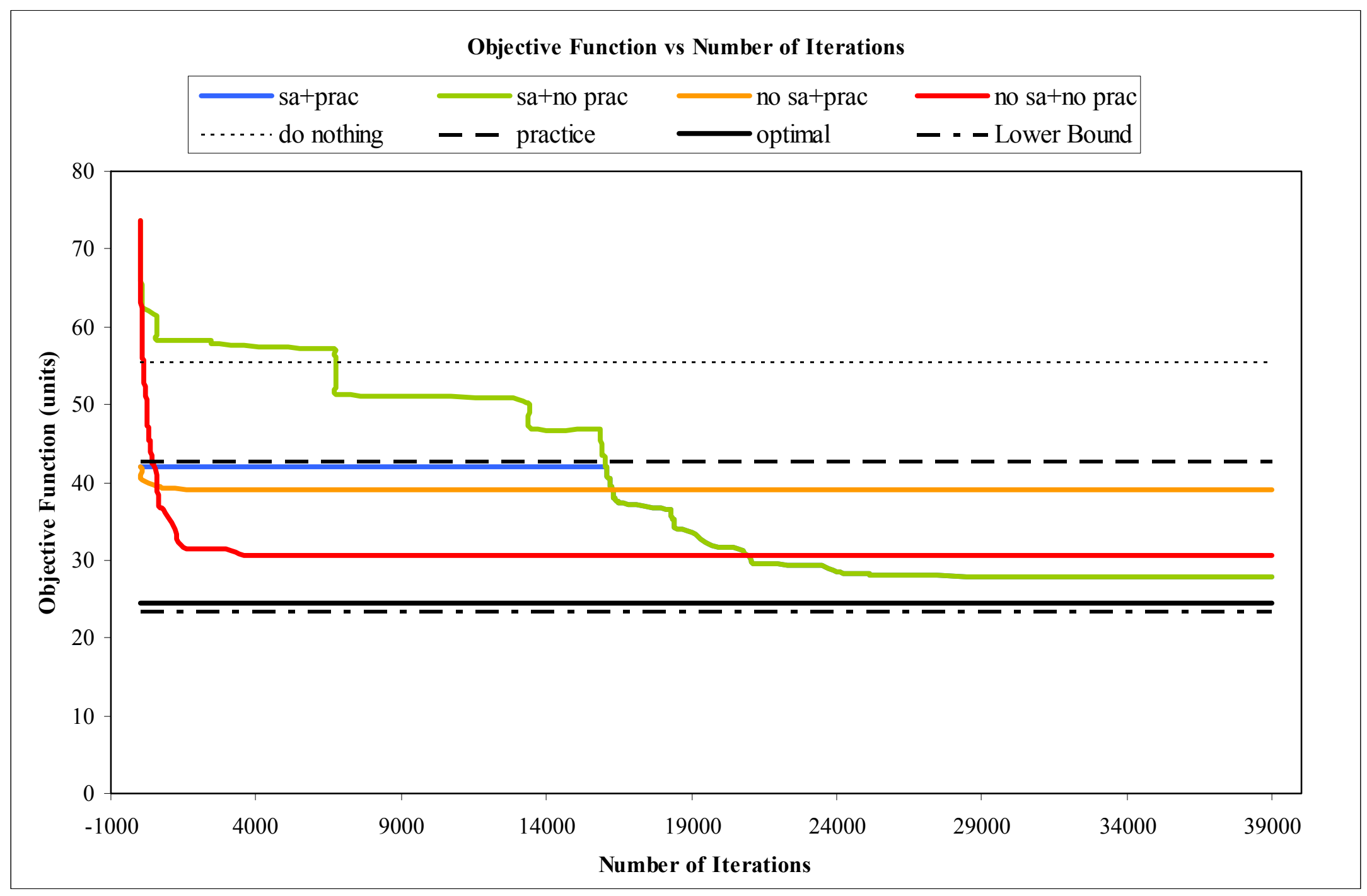

Figure 75 - Solution method comparison (Numerical experiment 2) 
Considering the literature-reported operation as the initial solution does not improve the quality of the solution. Furthermore, when the problem is solved using only the greedy heuristic search (without SA) the random initial solution seems to be a better option. Hence, the value of objective function for an initial solution cannot necessarily show the effectiveness of using that initial solution. Due to the large number of feasible solutions there is no guarantee that the optimal solution and a low cost initial solution are neighbors. However, since the problem is solved at the end of each planning horizon of every time a new demand arrives one suggestion would be to use the operation plan of last run as the initial solution for the new run. This suggestion is just for the future research and its effectiveness is not examined in this study.

Overall the quality of the solution obtained by using the heuristic search is not promising. As shown in Figure 75, even using the simulated annealing approach with 39,000 iterations $(140 \mathrm{sec}$.) the solution is no where close to the optimal solution. In an effort to further improve the quality of the solution, the third proposed solution algorithm is proposed.

Table 22 and Figures 76 and 77 present the result of solving the optimization problem for the 10-terminal network. The problem is solved under 7 different demand levels. The problem is solved using CPLEX, the proposed solution algorithm and the benchmarks. Execution times have been reported for exact solution method (CPLEX) and the path-based partitioning.

As discussed in the previous Chapter, the path-based partitioning method takes advantage of consolidation opportunities to avoid underutilizing the system's resources. The idea is to reduce the network size for each shipment by choosing only the links that appear in the first $\mathrm{K}$ shortest path for that particular shipment. Partitioning the network reduces the number of decision variables significantly. The results indicate that the path-based partitioning method outperforms other heuristic solution methods in terms of the quality of solution. Moreover, this method has a significant impact on the execution time reduction. Hence, it would be practical to use the exact solution methods to solve the modified problem for large size networks. 
Table 22 - Variable Total Shipment Size

\begin{tabular}{|c|c|c|c|c|c|c|c|c|c|c|}
\hline \multirow[b]{2}{*}{$\begin{array}{c}\text { Total } \\
\text { Shipment } \\
\text { Size } \\
\end{array}$} & \multirow[b]{2}{*}{$\begin{array}{c}\text { Number of } \\
\text { Variables }\end{array}$} & \multirow[b]{2}{*}{$\begin{array}{l}\text { Number of } \\
\text { Constraints }\end{array}$} & \multirow[b]{2}{*}{$\begin{array}{c}\text { CPLEX } \\
\text { Execution } \\
\text { Time (sec) } \\
\end{array}$} & \multirow[b]{2}{*}{$\begin{array}{c}\text { CPLEX with } \\
\text { Partitioning } \\
\text { Exec. Time (sec) }\end{array}$} & \multicolumn{6}{|c|}{ Total Operation Costs (units) } \\
\hline & & & & & $\begin{array}{l}\text { Lower } \\
\text { Bound }\end{array}$ & CPLEX & $\begin{array}{c}\text { CPLEX with } \\
\text { Path-based } \\
\text { Partitioning }\end{array}$ & $\begin{array}{c}\text { Path-based } \\
\text { Heuristic } \\
\text { Search }\end{array}$ & $\begin{array}{l}\text { Literature } \\
\text {-Reported } \\
\text { Operation } \\
\end{array}$ & Do-Nothing \\
\hline 10 & 3230 & 760 & 3 & 1 & 10.30 & 10.30 & 10.30 & 13.05 & 16.30 & 15.90 \\
\hline 20 & 5280 & 785 & 11 & 2 & 14.65 & 15.00 & 15.05 & 17.65 & 24.60 & 28.80 \\
\hline 30 & 6920 & 805 & 19 & 4 & 18.90 & 19.25 & 19.30 & 24.20 & 32.05 & 43.50 \\
\hline 40 & 9380 & 835 & 128 & 25 & 23.50 & 24.45 & 25.05 & 30.55 & 42.10 & 55.20 \\
\hline 50 & 11430 & 860 & 629 & 48 & 28.85 & 29.35 & 29.55 & 36.15 & 49.85 & 69.90 \\
\hline 60 & 13480 & 885 & 123 & 51 & 31.45 & 32.20 & 32.40 & 45.50 & 56.80 & 84.60 \\
\hline 70 & 15940 & 915 & 5700 & 590 & 35.55 & 38.25 & 38.45 & 52.70 & 73.05 & 99.30 \\
\hline
\end{tabular}

Note: Number of Time Periods $=9$, Number of Links $=20$, Number of Trucks $=8, \mathrm{WE}=1$ 


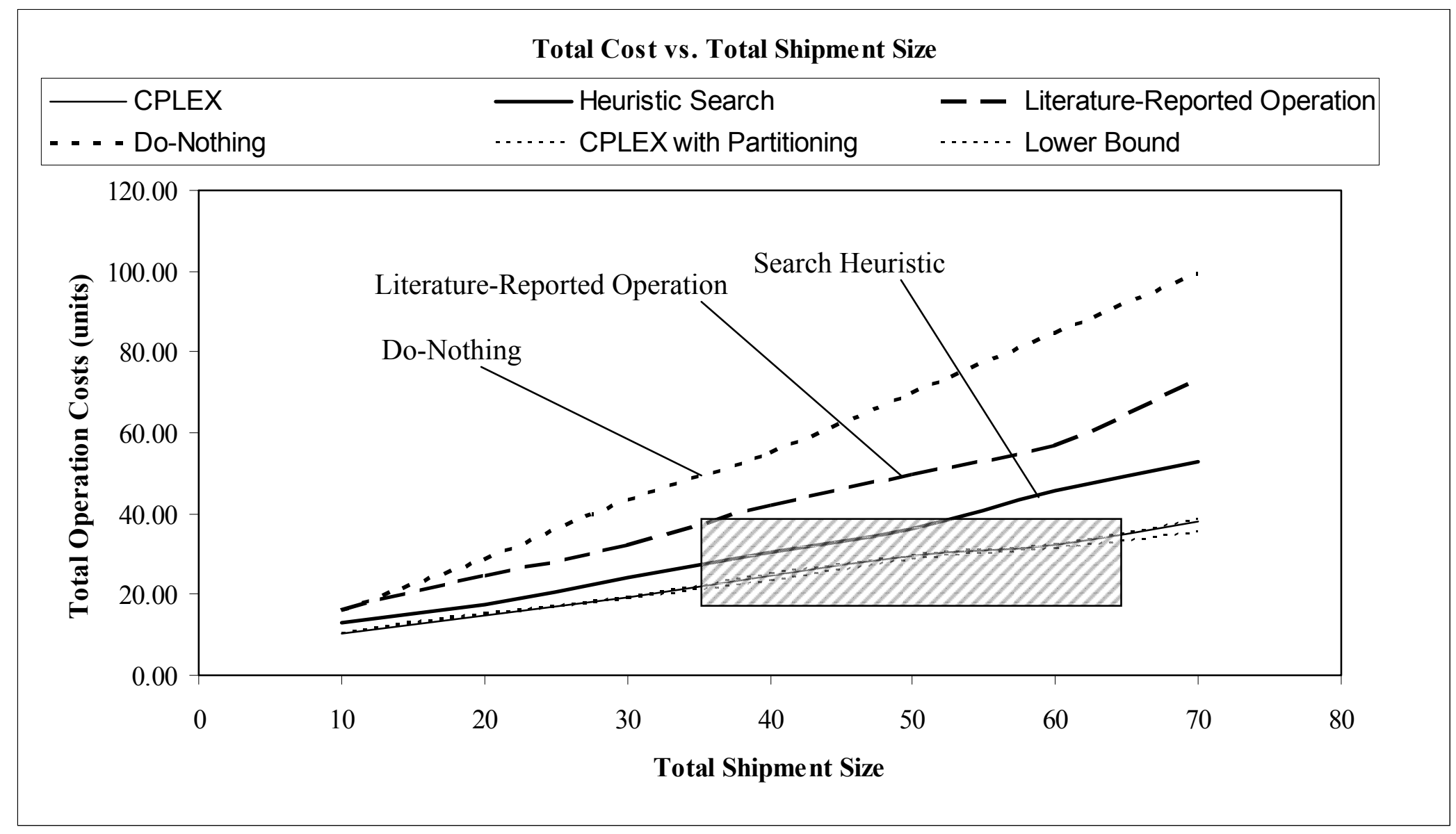

Figure 76 - Solution method comparison (Numerical experiment 2) 


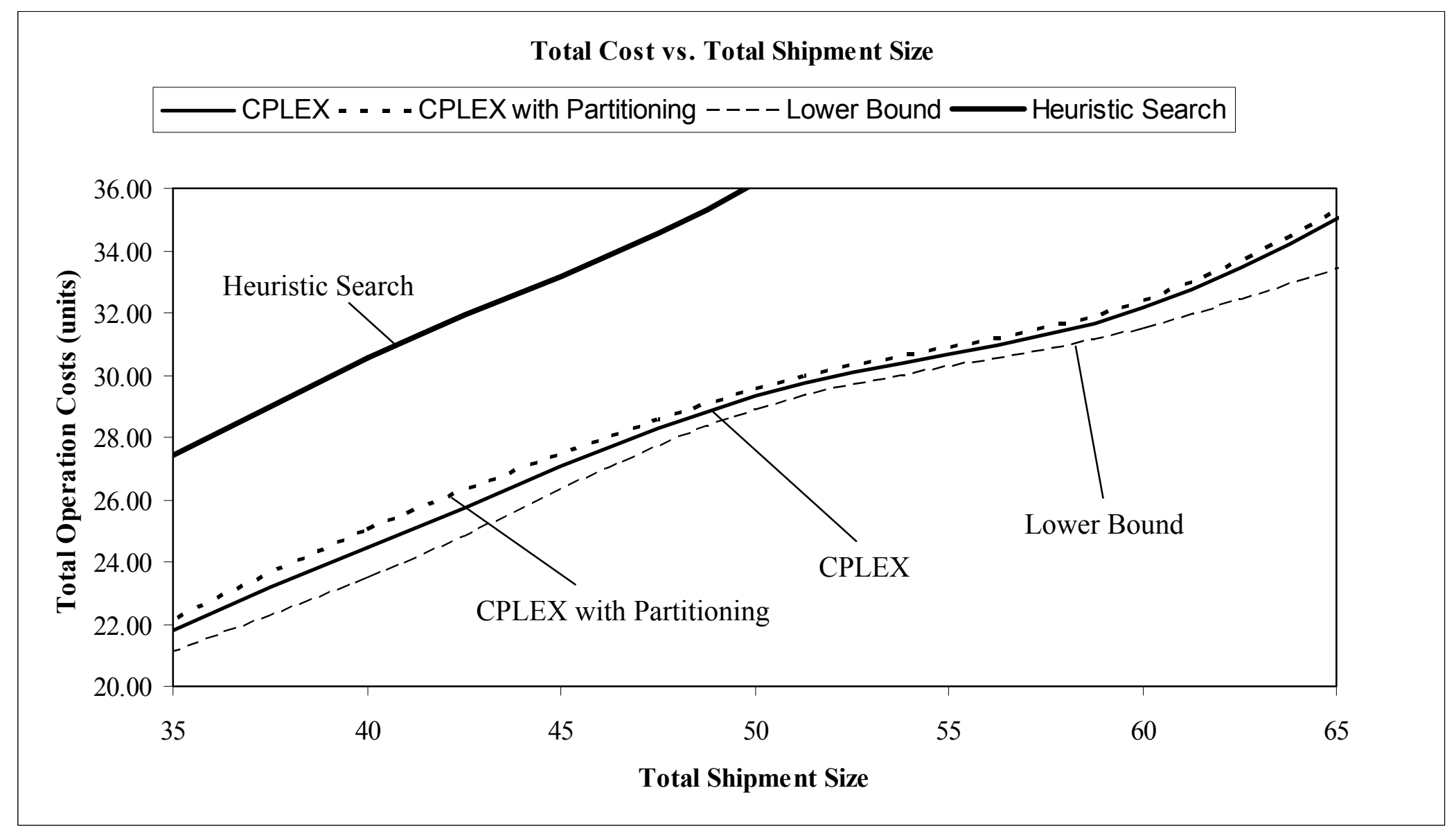

Figure 77 - Solution method comparison (Numerical experiment 2) 


\section{Network with 17 Breakbulks}

In the previous section, the performance of the proposed solution methods is analyzed using a mid-size network with 10 terminals. Three benchmark solutions were proposed to evaluate the effectiveness of the solution algorithm. The benchmarks are "do-nothing", "literature-reported operation" as upper bounds and also the "lower bound". The results indicate that the path-based partitioning method outperforms other heuristic solution methods in terms of the quality of solution. Moreover, this method has a significant impact on the execution time reduction.

In this section the performance of the proposed methods are analyzed using a more realistic large-size 17-terminal network. The main focus is to compare different versions of the path-based partitioning method with respect to the quality of solution and also the execution time. This section starts with the characteristics of the demand, the fleet and the network components. The discussion is followed by the results of the solution method comparison.

\section{- Realistic Case Study}

Trucking companies are trying to make more revenue by increasing their market share. It is important to know the characteristics of demand in different region to be able to focus company's services on customers' needs. Trucking companies do not reveal their markets and it is almost impossible to have any access to their demand OD matrix.

As discussed earlier in Chapter 5, the collection of trucking company financial and operating statistics (F\&OS) data is a mandatory program managed by the Bureau of Transportation Statistics (BTS). Motor carriers which have gross annual operating revenue of $\$ 3$ million or more are required to have annual reports, while carriers with revenues of $\$ 10$ million or more must also file four quarterly reports each year. Even in these reports the annual operating revenue or the total annual shipment size are mostly missing. 
In this section, 6 auto carriers are selected that were among class I carriers for 5 consecutive years from 1999 through 2003. The total annual operating expenses and total miles for these 5 carriers are available in BTS report. Using the available data for these 5 carriers and based on a series of assumptions the realistic case study is constructed. The data is used to evaluate the performance of the proposed solution algorithms.

The names, numbers and the States of the selected auto carriers are listed in Table 22. The information regarding the service area and number of hubs are available for some companies from their website. Pacific Motor and Jack Cooper are among top auto carrier companies that provide service to as many as 26 States.

The operational revenue and miles traveled per year are the average of numbers that were reported by companies from 1999 to 2003 [6]. Based on the online quotes listed in Table 7, it is assumed that the delivery of each shipment (car) has average operational revenue of $\$ 600$ for the company. Therefore the average number of shipments that are being delivered per day can be calculated. It is also assumed that each truck can travel 605 miles per day on average (considering 11 working hours for driver and $55 \mathrm{miles} / \mathrm{hr}$ average speed). Based on these assumptions and using the average operational miles per day one can calculate the average number of trucks that are operating on a daily basis. Furthermore, the average number of shipments per truck can be calculated. The calculated values appear in last 4 rows in Table 23. According to shipment/truck value, Ewing Bro Inc's operation was more efficient than other companies. They generated more than $\$ 7$ revenue for each mile traveled by their trucks.

The number of requests for these top auto transport companies can go up to a maximum of 500 shipments per day. As discussed earlier, around $60-80 \%$ of this demand has a high degree of certainty. This part of demand mostly includes new vehicles that must be shipped from assembly plants to dealers/customers or between dealers. The uncertain portion of demand mostly consists of pre-owned vehicles transported between dealers, auctions, or private parties (e.g. online sellers and buyers). 
Using tactical planning a carrier can manage that part of demand that has a high degree of certainty. They solve the network design problem in order to find the direct service links. A minimum frequency is maintained on each link and the shipment requests are handled by implementing the minimum fill-rate requirements, holding time limits and go-when-fill policies. Based on the information presented in Table 22 and considering the worst possible scenario where $40 \%$ of demand is revealed dynamically, up to 200 shipments per day must be handled using the proposed dynamic decision making based on the online shipment/truck routing and scheduling. 
Table 23 - Class I Auto Carriers (1999-2003)

\begin{tabular}{|c|c|c|c|c|c|c|}
\hline $\begin{array}{l}\text { Motor } \\
\text { Carrier } \\
\text { Company }\end{array}$ & $\begin{array}{c}\text { EWING } \\
\text { BROS } \\
\text { INC }\end{array}$ & $\begin{array}{c}\text { DMT } \\
\text { TRUCKING } \\
\text { INC }\end{array}$ & $\begin{array}{c}\text { AUTOMOBILE } \\
\text { TRANSPORT } \\
\text { SPECIALISTS } \\
\text { INC }\end{array}$ & $\begin{array}{c}\text { SELLAND } \\
\text { AUTO } \\
\text { TRANSPORT } \\
\text { INC }\end{array}$ & $\begin{array}{l}\text { PACIFIC } \\
\text { MOTOR } \\
\text { TRUCKING } \\
\text { COMPANY }\end{array}$ & $\begin{array}{c}\text { JACK } \\
\text { COOPER } \\
\text { TRANSPORT } \\
\text { COMPANY }\end{array}$ \\
\hline Motor Carrier \# & 117380 & 148860 & 153385 & 134614 & 215978 & 71902 \\
\hline City & $\begin{array}{c}\text { LAS } \\
\text { VEGAS }\end{array}$ & BALTIMORE & AURORA & SEATTLE & $\begin{array}{l}\text { KANSAS } \\
\text { CITY }\end{array}$ & $\begin{array}{l}\text { KANSAS } \\
\text { CITY }\end{array}$ \\
\hline State & NV & MD & $\mathrm{CO}$ & WA & MO & MO \\
\hline Number of States & NA & NA & NA & 11 & 26 & 26 \\
\hline Number of Hubs & NA & NA & NA & 7 & NA & NA \\
\hline $\begin{array}{l}\text { Operational } \\
\text { Revenue (\$) }\end{array}$ & $9,130,979$ & $9,235,600$ & $10,092,152$ & $25,419,151$ & $59,259,023$ & $121,375,537$ \\
\hline Miles & $1,281,307$ & $3,769,856$ & $4,036,672$ & $10,802,771$ & $15,859,207$ & $42,277,163$ \\
\hline Shipments / Day & 42 & 42 & 46 & 116 & 271 & 554 \\
\hline Miles / Day & 3510 & 10328 & 11059 & 29597 & 43450 & 115828 \\
\hline Number of Trucks & 6 & 17 & 18 & 49 & 72 & 191 \\
\hline Shipments / Truck & 7.2 & 2.5 & 2.5 & 2.4 & 3.8 & 2.9 \\
\hline
\end{tabular}


The average loading/unloading time for a truck is estimated to be around 20 minutes. Therefore, the total loading/unloading for a 10-car auto carrier truck is considered to be about 6 hours, which is selected as the length of one time period in the time-space network. Considering the 200 shipments per day assumption, the average demand for each time period would be equal to 50 cars. The solution method comparison is performed based on the above assumptions.

The next step is to construct the network for the realistic case study, and the main goal would be to cover the largest possible area throughout the United States. Hubs (break-bulk terminals) were allocated near major cities. Microsoft Streets and Trips software is used to ensure that the actual highway mileage between each 2 terminals meets the driver working hour requirements. Several combinations of node locations and service links were examined.

Figure 78 shows the final design of the hub-and-spoke network, which consists of 17 terminal and 36 links. Note that, these are the main hubs of the network. Satellite terminals operate regionally around each break-bulk terminal. Those are the main origins and destinations for the shipments. The loads (cars) are being hauled between these satellites and primary break-bulk terminals using smaller trailers (e.g. hotshots, flat-bed, drop-decks...).

Clearly, when satellite terminals are considered as a part of network, a more realistic problem is generated. However, solving the resulting problem is beyond the scope of this study. In the last Chapter of this dissertation, this issue is addressed and recommendations are provided for future studies on dealing with the local pickup and delivery problem in conjunction with the shipment and truck routing problem for the hub-and-spoke network. The main focus of this study is to solve the shipment and truck routing and scheduling problem on the hub network.

Based on the estimates provided in Table 23, it is assumed that there are 17 auto carrier trucks available over the region, one truck at each hub/terminal. The fleet is assumed to be homogeneous and all characteristics of trucks are the same. The next section of this Chapter discusses the effect of considering the non-homogeneous fleet on the complexity of the mathematical model. 


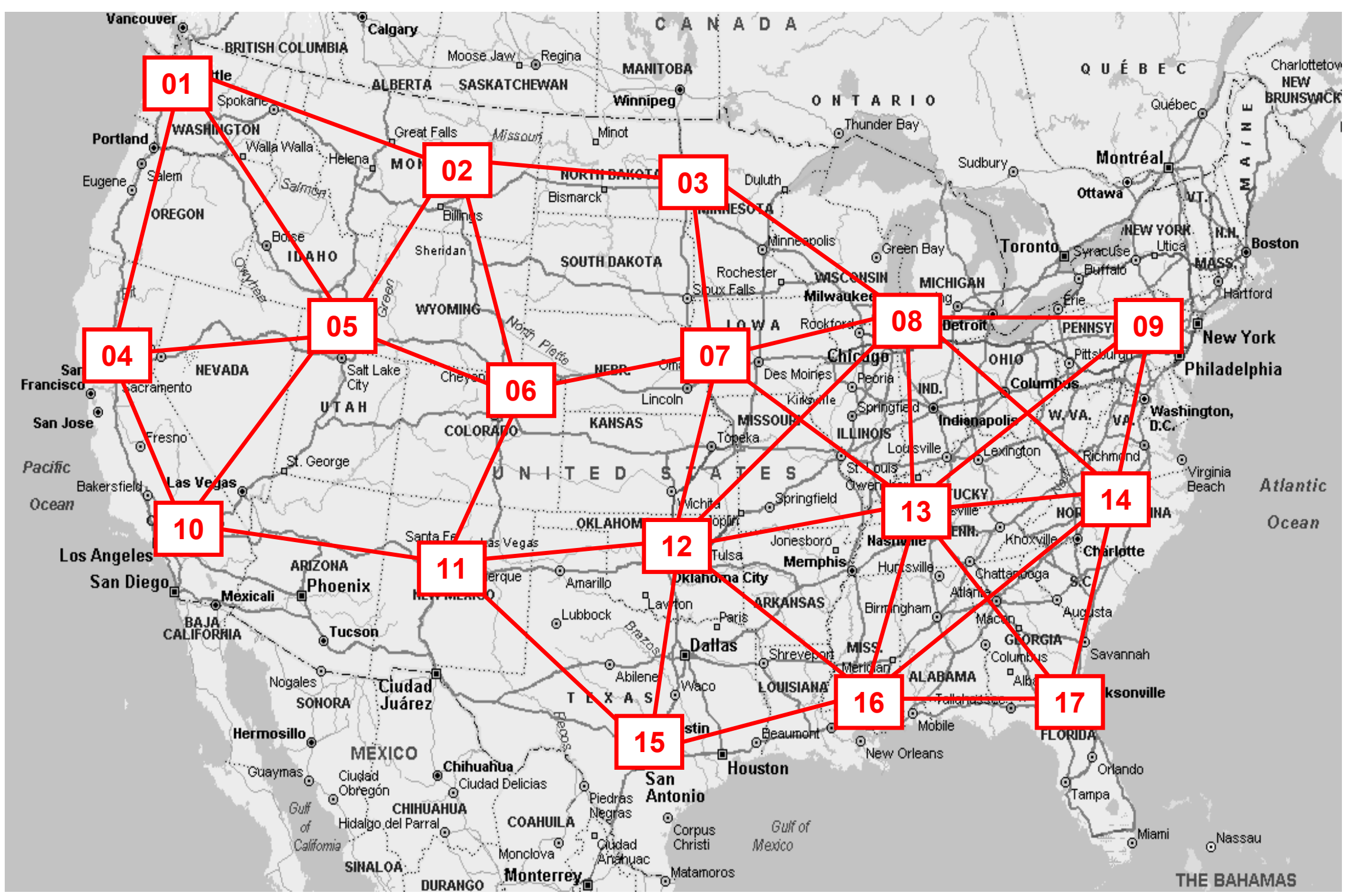

Figure 78 - Physical network (17-terminal) 
- Solution Method Comparison

As discussed earlier, in this section a large-size problem is used to evaluate the performance of the proposed solution algorithms. In decision making procedure the combined shipment and truck routing problem is solved repeatedly. In the previous Chapter three different solution algorithms were proposed to solve the largesize MIP problem when using the exact solution methods is not practical. The results of the numerical experiments on a mid-size problem suggest that the path-based partitioning method outperforms other heuristic solution methods in terms of the quality of solution. This method has a significant impact on the execution time reduction.

This section mainly focuses on different versions of the path-based partitioning method. The numerical experiment is conducted using a Pentium M (1.60GHz) machine. By running the "Operation Plan" program the performance measures are calculated and used as the basis for the analysis and solution method comparison. Similar to the previous experiments on the 10 -terminal problem, the proposed benchmarks; “do-nothing”, "literature-reported operation" as upper bounds and also a "lower bound" are used to evaluate the effectiveness of the solution algorithms. CPLEX cannot be used to solve the problem to optimality since the branch-and-cut trees become really large and getting the insufficient memory errors cannot be prevented even by setting the memory parameters. Hence, the execution time and the solution gap limit parameters have been set to manage the CPLEX running time. The results are used as additional benchmarks.

As the $3^{\text {rd }}$ proposed method to solve the mathematical problem, a path-based network partitioning is used in conjunction with CPLEX. The idea is to reduce the network size for each shipment by choosing only the links that appear in the first $\mathrm{K}$ shortest path for that particular shipment. Partitioning the network reduces the number of decision variables significantly. Hence, it would be practical to use the exact solution methods to solve the resulting modified MIP problem for large size networks. The maximum reduction in network size is obtained by using only one path 
to partition the network and the resulting network is called "SP-1". In general SP-K represents a partitioned network containing only the $\mathrm{K}$ first shortest paths for each shipment. Naturally, considering more paths in the modified network improves the solution quality. However, the computation time also increases. Therefore, a set of preliminary numerical experiment is required to maintain a balance between solution quality and execution time by finding the optimum number of shortest paths to be used in the network partitioning process.

Table 24 and Figure $79-82$ present the result of solving the problem for the 17-terminal network. As discussed in the previous Chapter, using simulated annealing combined with the heuristic search improves the solution quality, but it has a relatively high execution time. Therefore this method is excluded from the list of candidate solution methods that is considered in this section. Here is the list of solution methods that are compared:

- Heuristic search without simulated annealing

- SP-K (for K from 1 through 7)

- SP-8* (SP-8 considering paths that are longer than the average)

As discussed in the previous Chapter, the path-based partitioning method takes advantage of consolidation opportunities to avoid underutilizing the system's resources. The network size is reduced for each shipment by choosing only the links that appear in the first $\mathrm{K}$ shortest path for that particular shipment. Partitioning the network reduces the number of decision variables. As shown in Table 24, even the largest reduced-size problem (SP-7) can be solved using CPLEX in less than 2 hours, but the actual MIP problem cannot be solved in 28 hours.

The quality of the solution improves by including more paths in the pathbased partitioning method. However, considering more paths is computationally expensive. As shown in Table 24, using SP-7 would result a 1\% reduction in total cost compared to SP-4, while the execution time of using SP-7 is 20 times more than that of SP-4. Therefore, the best level of partitioning must be picked to solve the problem considering the execution time constraint to solve the problem. 
Based on the results obtained from this numerical experiment, it seems that SP-4 would be the most efficient choice in this example. The quality of its solutions is acceptable compare to the results obtained from CPLEX with $<5 \%$ gap. Moreover, its execution time guarantees that the solution method can be used for dynamic application. 
Table 24 - Solution Methods

\begin{tabular}{|c|c|c|c|c|c|c|c|c|c|c|c|c|c|c|c|}
\hline $\begin{array}{l}\text { Performance } \\
\text { Measure }\end{array}$ & $\begin{array}{c}\text { Do- } \\
\text { Nothing }\end{array}$ & $\begin{array}{l}\text { Literature } \\
\text {-Reported } \\
\text { Operation }\end{array}$ & $\begin{array}{l}\text { Heuristic } \\
\text { Search }\end{array}$ & $\begin{array}{l}\text { CPLEX } \\
\text { with } \\
\text { SP 1 }\end{array}$ & $\begin{array}{l}\text { CPLEX } \\
\text { with } \\
\text { SP } 2\end{array}$ & $\begin{array}{l}\text { CPLEX } \\
\text { with } \\
\text { SP } 3\end{array}$ & $\begin{array}{l}\text { CPLEX } \\
\text { with } \\
\text { SP } 4\end{array}$ & $\begin{array}{l}\text { CPLEX } \\
\text { with } \\
\text { SP } 5\end{array}$ & $\begin{array}{l}\text { CPLEX } \\
\text { with } \\
\text { SP } 6\end{array}$ & $\begin{array}{l}\text { CPLEX } \\
\text { with } \\
\text { SP } 7\end{array}$ & $\begin{array}{l}\text { CPLEX } \\
\text { with } \\
\text { SP 8* }\end{array}$ & $\begin{array}{l}\text { CPLEX } \\
(600 \\
\text { sec })\end{array}$ & $\begin{array}{c}\text { CPLEX } \\
(7200 \\
\text { sec })\end{array}$ & $\begin{array}{c}\text { CPLEX } \\
(<5 \% \\
\text { gap })\end{array}$ & $\begin{array}{l}\text { Lower } \\
\text { Bound }\end{array}$ \\
\hline $\begin{array}{l}\text { \# of Loaded } \\
\text { Truck Moves }\end{array}$ & 0 & 46 & 64 & 52 & 52 & 46 & 40 & 40 & 40 & 40 & 44 & 48 & 42 & 40 & \\
\hline $\begin{array}{l}\text { \# of Empty Truck } \\
\text { Moves }\end{array}$ & 0 & 0 & 2 & 8 & 2 & 4 & 2 & 2 & 2 & 2 & 2 & 4 & 2 & 0 & \\
\hline $\begin{array}{l}\text { \# of Loaded } \\
\text { Truck Trips }\end{array}$ & 0 & 23 & 32 & 26 & 26 & 23 & 20 & 20 & 20 & 20 & 22 & 24 & 21 & 20 & \\
\hline $\begin{array}{l}\text { \# of Empty Truck } \\
\text { Trips }\end{array}$ & 0 & 0 & 1 & 4 & 1 & 2 & 1 & 1 & 1 & 1 & 1 & 2 & 1 & 0 & \\
\hline $\begin{array}{l}\text { Loaded Truck } \\
\text { Routing Cost } \\
\text { (units) }\end{array}$ & 0.00 & 55.20 & 76.80 & 62.40 & 62.40 & 55.20 & 48.00 & 48.00 & 48.00 & 48.00 & 52.80 & 57.60 & 50.40 & 48.00 & \\
\hline $\begin{array}{l}\text { Empty Truck } \\
\text { Routing Cost } \\
\text { (units) }\end{array}$ & 0.00 & 0.00 & 40.00 & 1.60 & 0.40 & 0.80 & 0.40 & 0.40 & 0.40 & 0.40 & 0.40 & 0.80 & 0.40 & 0.00 & \\
\hline $\begin{array}{l}\text { Average } \\
\text { Fill Rate (\%) }\end{array}$ & 0 & 26 & 32 & 38 & 39 & 44 & 55 & 55 & 55 & 57 & 48 & 47 & 56 & 59 & \\
\hline $\begin{array}{l}\text { \# of Shipments } \\
\text { Delivered }\end{array}$ & 0 & 30 & 44 & 46 & 49 & 47 & 46 & 46 & 46 & 47 & 47 & 48 & 45 & 45 & \\
\hline $\begin{array}{l}\text { \# of Shipments } \\
\text { Not-Delivered }\end{array}$ & 50 & 20 & 6 & 4 & 1 & 3 & 4 & 4 & 4 & 3 & 3 & 2 & 5 & 5 & \\
\hline $\begin{array}{l}\text { Carrier's Cost } \\
\text { (units) }\end{array}$ & 50.00 & 78.15 & 89.60 & 75.90 & 71.90 & 66.10 & 60.85 & 60.85 & 60.85 & 60.35 & 64.75 & 71.50 & 61.05 & 58.15 & \\
\hline $\begin{array}{l}\text { Customers' Cost } \\
\text { (units) }\end{array}$ & 91.80 & 38.70 & 10.95 & 6.30 & 0.90 & 4.50 & 4.70 & 4.70 & 4.70 & 4.50 & 4.80 & 3.20 & 5.10 & 5.90 & \\
\hline Total Cost (units) & 141.80 & 116.85 & 100.55 & 82.20 & 72.80 & 70.60 & 65.55 & 65.55 & 65.55 & 64.85 & 69.55 & 74.70 & 66.30 & 64.05 & 63.30 \\
\hline $\begin{array}{l}\text { Execution Time } \\
\text { (sec) }\end{array}$ & 0 & 0 & 231 & 1 & 594 & 224 & 223 & 834 & 1301 & 4675 & 3851 & 600 & 7200 & 102792 & 437 \\
\hline $\begin{array}{l}\text { Time to reach the } \\
\text { best solution (sec) }\end{array}$ & & & & 1 & 94 & 165 & 50 & 670 & 450 & 780 & 760 & & & & \\
\hline $\begin{array}{l}\text { CPLEX Best } \\
\text { Bound (units) }\end{array}$ & & & & & & & & & & & & 59.15 & 59.37 & 61.07 & \\
\hline CPLEX Gap (\%) & & & & & & & & & & & & 21 & 11 & 5 & \\
\hline
\end{tabular}

Note: Number of Terminals=17, Number of Time Periods $=20$, Number of Links $=36$, Number of Trucks $=17$, Number of Shipments $=50$ 


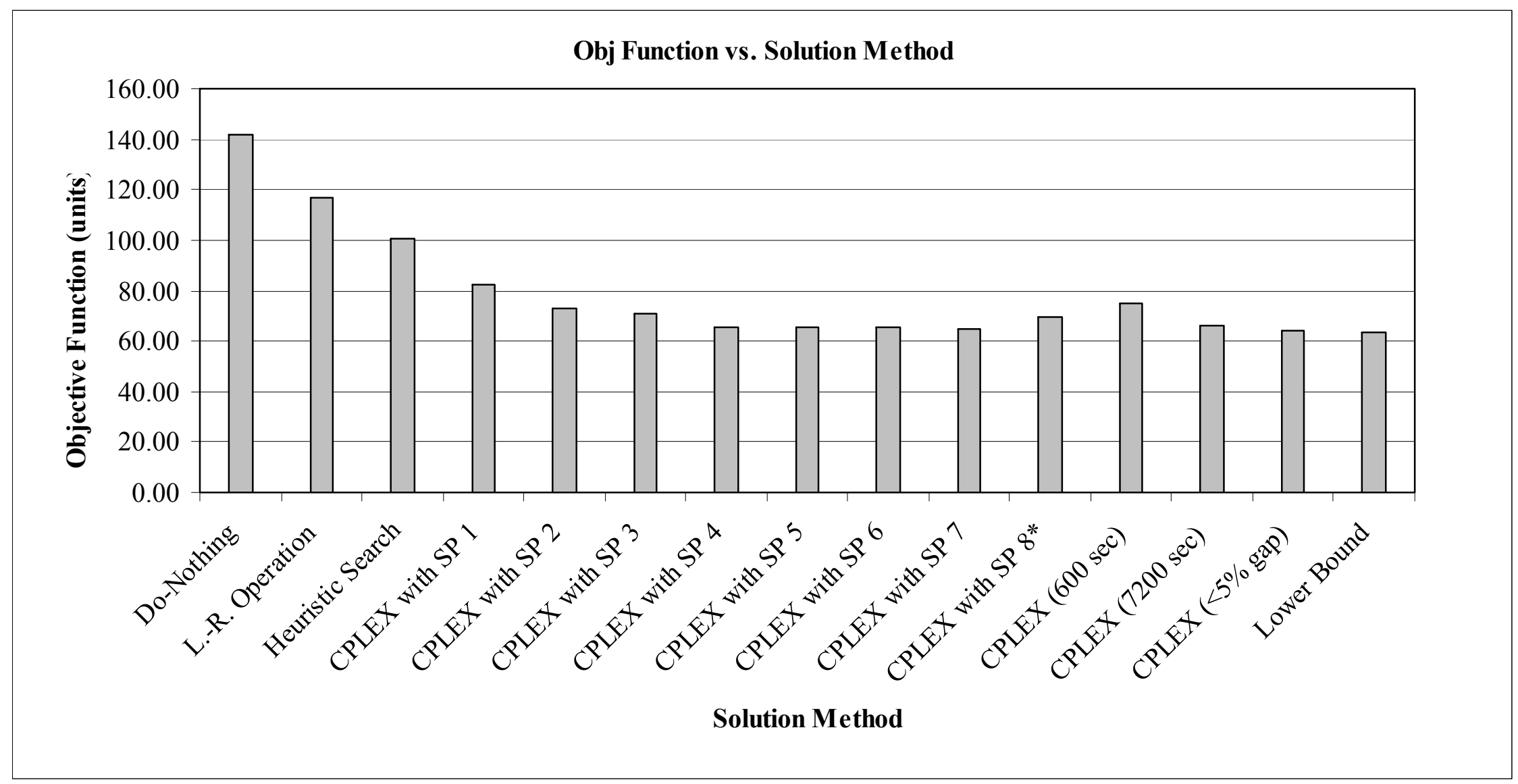

Figure 79 - Solution method comparison based on the objective function (Numerical experiment 3) 


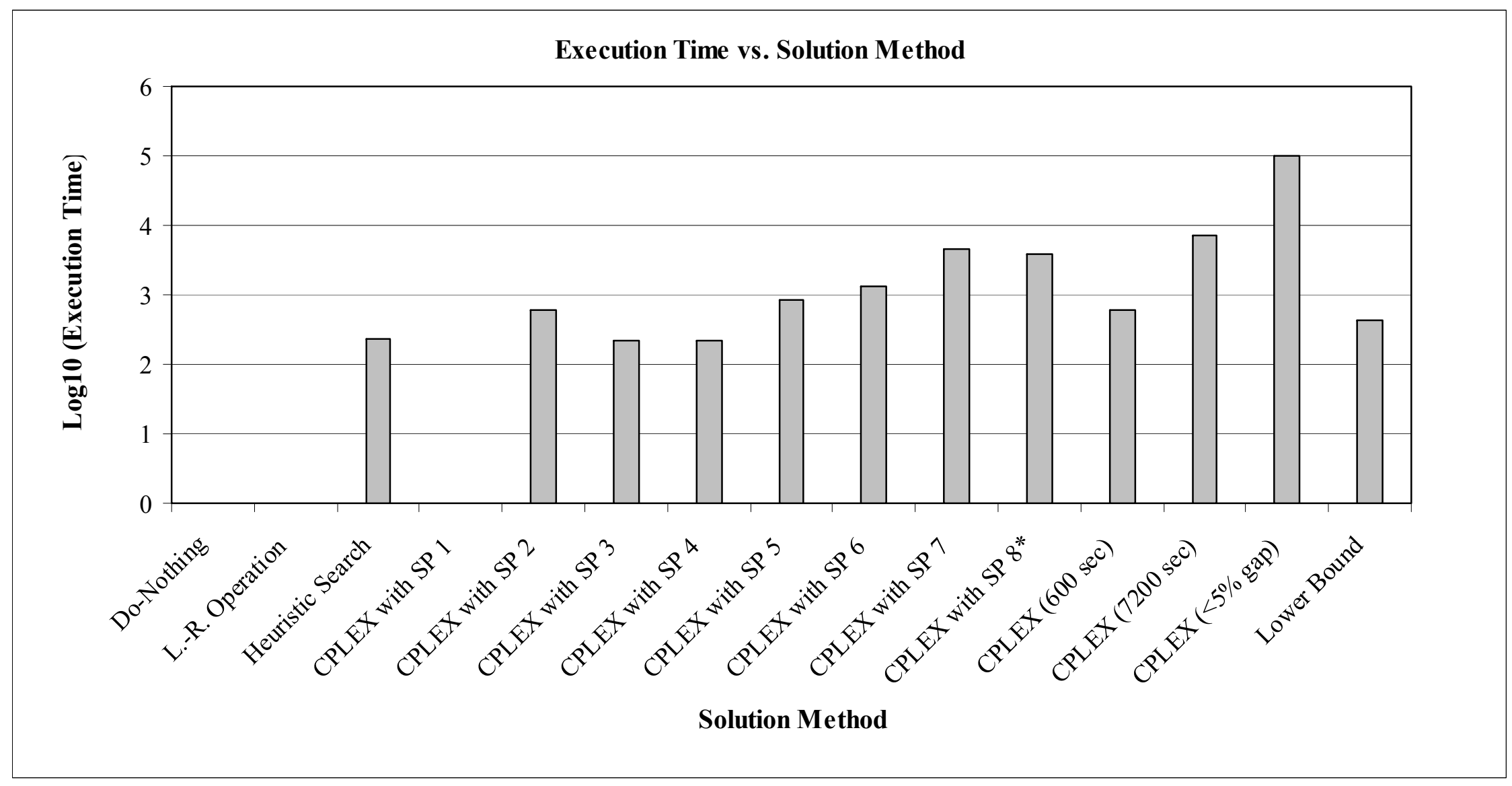

Figure 80 - Solution method comparison based on the execution time (Numerical experiment 3 ) 


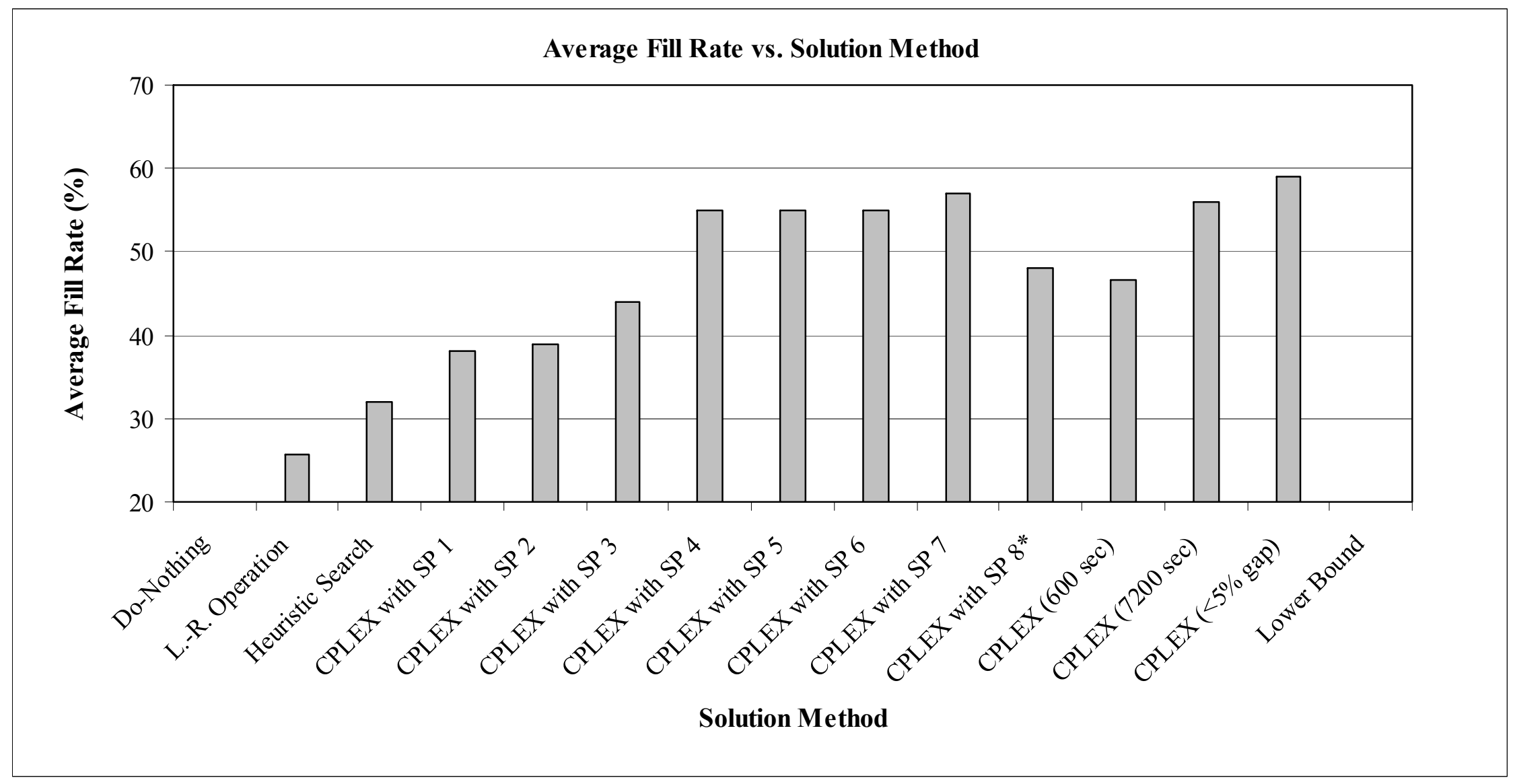

Figure 81 - Solution method comparison based on the average fill rate (Numerical experiment 3) 


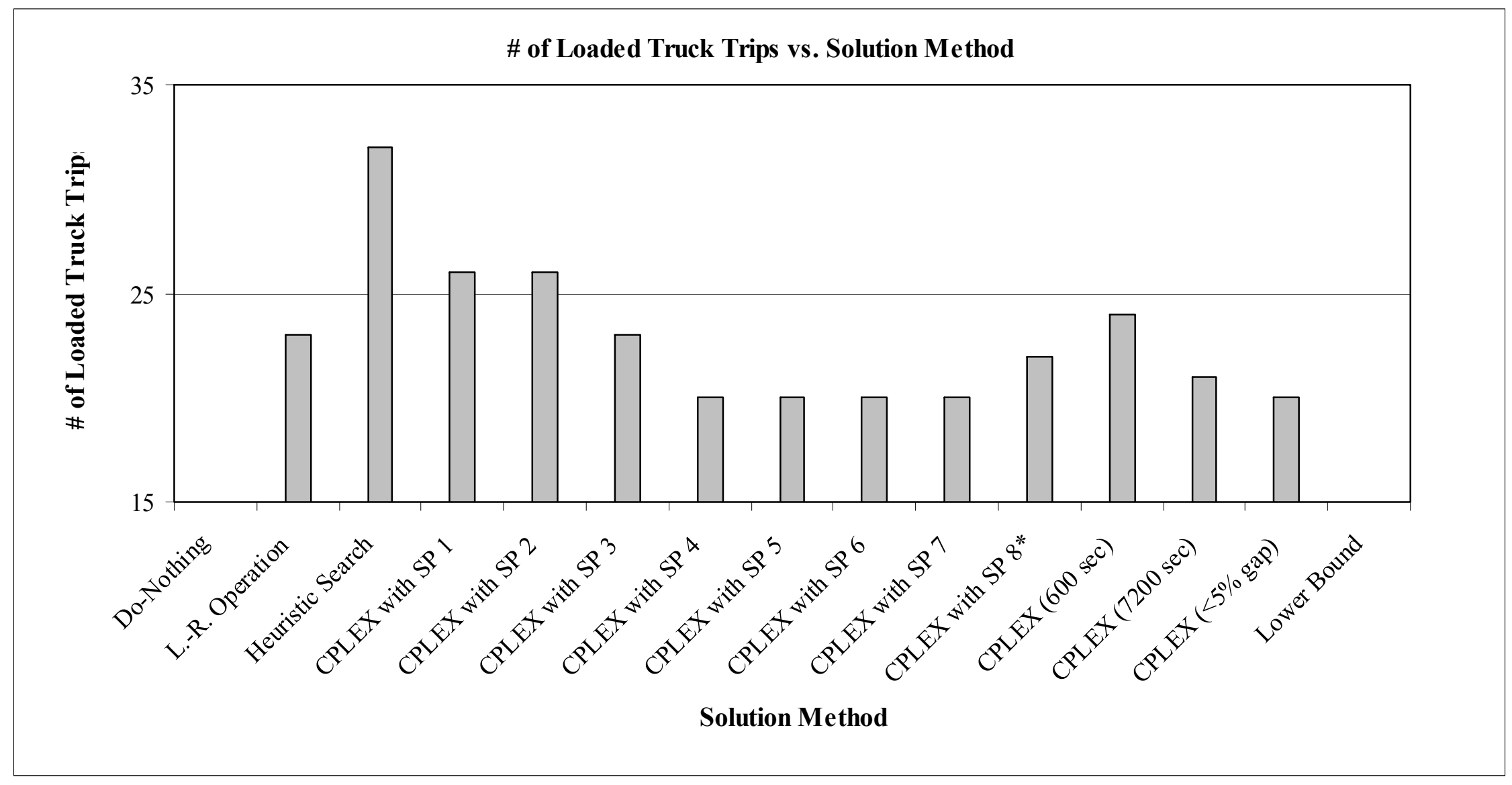

Figure 82 - Solution method comparison based on the number of loaded truck trips (Numerical experiment 3 ) 
As shown in Table 24 and Figure 80, the execution time of CPLEX with SP2 is more than that of CPLEX with SP3. This is an unexpected result since SP2 is a much smaller problem than SP3. However, as shown in Table 24, CPLEX finds the best solution for SP2 in only 94 minutes. Then it takes more time to search all solutions in branch-and-bound tree to ensure that the solution is optimal. In case of $\mathrm{SP} 3$, the convergence is faster due to the structure of the problem and the number of branches that cancels out implicitly.

The results of the numerical experiments on a large-size problem indicate that all different versions of the path-based partitioning method outperform the heuristic search algorithm in all the following aspects:

- The quality of solution is higher, which means that there will be less customer associated cost (customer satisfaction) and less carrier associated cost (more profit).

- The execution time is less than other methods, which means that the proposed method is capable of being used in a dynamic setting.

- The average fill rates are higher, which shows that using this method the company can avoid underutilizing its resources.

- The number of loaded truck movements is less, which leads the company to reduce all the operating costs that haven't been considered in the objective function of the mathematical model. 
- Non-Homogeneous Fleet

As it is discussed in Chapter 3, fleet can be treated as a commodity in the proposed MCNF model. Therefore, in order to capture the non-homogeneous fleet condition, one commodity must be added for each type of trucks. Chapter 3 addressed the issue by introducing a modified version of the proposed mathematical model.

This section describes the impact of non-homogeneous fleet assumption on the size of the optimization model, and the computation time that is required to solve the problem. In the base case of the 17-terminal problem, 17 similar trucks are available at hubs ( 1 truck at each terminal) to deliver 50 shipments. In order to compare the non-homogeneous fleet case against the homogeneous fleet case, a variation of the base case is constructed. In this slightly modified instance of the base case, the fleet of 17 trucks is divided into 4 different types of truck. All features of these 4 types of trucks are the same, except their colors! All the other characteristics of the problem with non-homogeneous fleet are the same as the problem with homogeneous fleet.

To generate the mathematical problem for non-homogeneous case, the "Problem Generator" C program is modified by adding the additional cost function and constraints. As shown in Figure 83, a new input file contains the information related to each truck type; the capacity, minimum fill rate, and associated cost factors. Also, the modified empty truck input file reflects the type of the truck that is available at each terminal before the operation starts.

The "Operation Plan" C program is also modified. As shown in Figure 84 and 77, the new version of two processed output files that show the performance measures and truck dispatch plans contain information associated with each truck type. Both problems are solved by CPLEX using the path-based partitioning (SP3). The results are presented in Table 25. As a result of dividing the fleet into 4 types, the number of variables and constraints related to loaded and empty trucks are multiplied by 4 . 
- Fleet Information:

$\begin{array}{ccccc}\text { Truck Type } & \text { WF } & \text { WE } & C F & C E \\ -1 & 10 & 0 & 1.20 & 0.20 \\ 2 & 10 & 0 & 1.20 & 0.20 \\ 3 & 10 & 0 & 1.20 & 0.20 \\ 4 & 10 & 0 & 1.20 & 0.20\end{array}$

- Empty Truck Locations:

$\begin{array}{cccc}\text { Termina } 1 & \text { Time } & \text { Truck Type Number } & \text { of Empty Trucks } \\ -1 & 0 & 1 & 1 \\ \frac{1}{2} & 0 & 2 & 1 \\ 3 & 0 & 2 & 1 \\ 4 & 0 & 3 & 1 \\ 5 & 0 & 3 & 1 \\ 6 & 0 & 4 & 1 \\ 7 & 0 & 2 & 1 \\ 8 & 0 & 1 & 1 \\ 9 & 0 & 3 & 1 \\ 10 & 0 & 1 & 1 \\ 11 & 0 & 1 & 1 \\ 12 & 0 & 2 & 1 \\ 13 & 0 & 4 & 1 \\ 14 & 0 & 1 & 1 \\ 15 & 0 & 3 & 1 \\ 16 & 0 & 2 & 1 \\ 17 & 0 & 2 & 1\end{array}$

Figure 83 - Fleet information (Non-homogeneous fleet) 


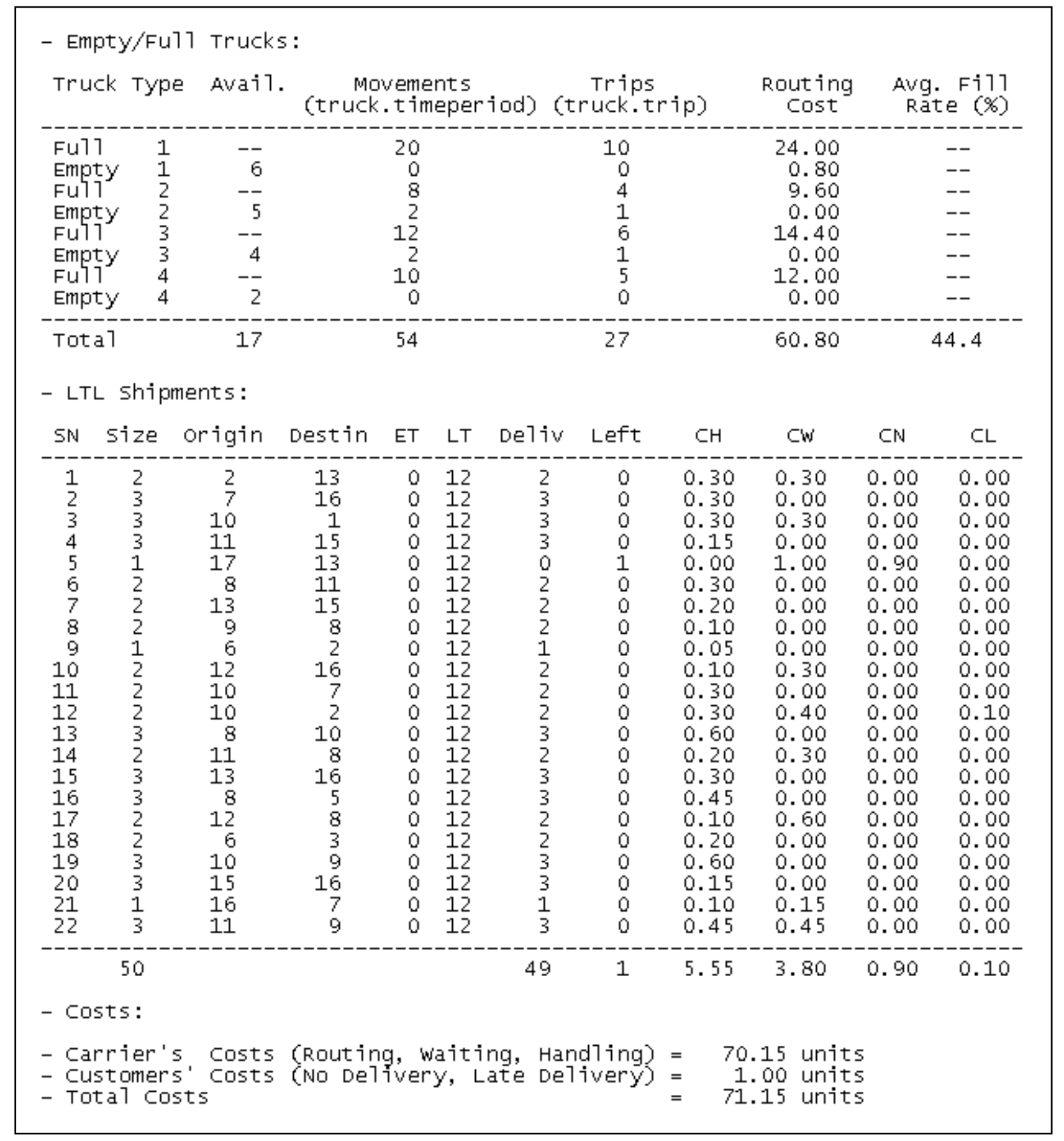

Figure 84 - Performance measures (Non-homogeneous fleet) 


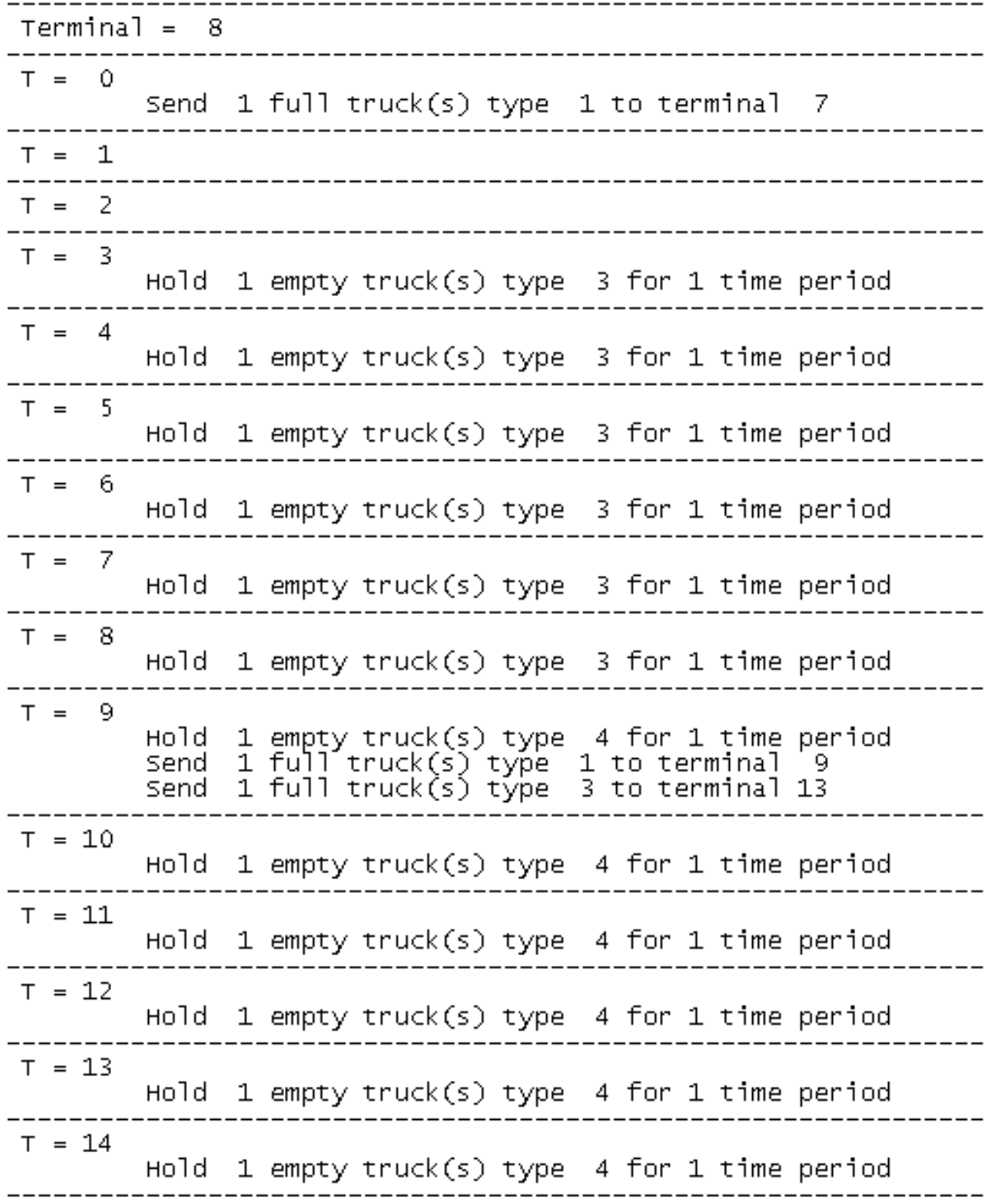

Figure 85 - Truck dispatch plan (Numerical experiment 1) 
Table 25 - Non-Homogeneous Fleet

\begin{tabular}{lcc}
\hline Measure & $\begin{array}{c}\text { Homogeneous Fleet } \\
\text { (CPLEX with SP 3) }\end{array}$ & $\begin{array}{c}\text { Non-Homogeneous Fleet } \\
\text { (CPLEX with SP 3) }\end{array}$ \\
\hline Number of Shipment Variables & 35992 & 35992 \\
Number of Loaded Truck Variables & $\mathbf{1 2 9 6}$ & $\mathbf{5 1 8 4}$ \\
Number of Empty Truck Variables & $\mathbf{1 7 0 8}$ & $\mathbf{6 8 3 2}$ \\
Total Number of Variables & $\mathbf{3 8 9 9 6}$ & $\mathbf{4 8 0 0 8}$ \\
\hline Number of Shipment Conservation Constraints & 110 & 110 \\
Number of Truck Conservation Constraints & $\mathbf{3 4 0}$ & $\mathbf{1 3 6 0}$ \\
Number of Shipment-Truck Constraints & 2592 & 2592 \\
Total Number of Constraints & $\mathbf{3 0 4 2}$ & $\mathbf{4 0 6 2}$ \\
\hline Number of Loaded Truck Trips & 23 & 25 \\
Number of Empty Truck Trips & 2 & 2 \\
Average Fill Rate (\%) & 44 & 44 \\
Number of Shipments Delivered & 47 & 49 \\
Number of Shipments Not-Delivered & 3 & 1.00 \\
\hline Carriers' Associated Costs & 66.10 & 71.15 \\
Customers Associated Costs & 4.50 & $\mathbf{7 8 7}(<\mathbf{5 \%}$ gap) \\
Total Cost & 70.60 & \\
\hline Execution Time (sec) & $\mathbf{2 2 4}$ & $\mathbf{2}$ \\
\hline
\end{tabular}

Note: Number of Terminals=17, Number of Time Periods $=20$, Number of Links $=36$, Number of Trucks $=17$, Number of Shipments $=50$, Type of Trucks (Only For Non-Homogeneous Fleet) $=4$ 
Using CPLEX with the proposed path-based partitioning (SP3) an acceptable solution ( $<5 \%$ gap) can be obtained in a relatively short time ( $787 \mathrm{sec}$.). However, due to the lack of the available memory, even this problem cannot be solved using CPLEX up to optimality. For this numerical experiment the maximum possible memory is used by adjusting the WORKMEM parameter in CPLEX. In spite of the adjustment, CPLEX stops due to "running out of memory" error after 8233 seconds of running time. There is no improvement in the solution, and the gap between best bound and the best solution is still more than $4 \%$. This shows the increased complexity of the problem after applying the non-homogenous fleet assumption. As shown in Table 24, the homogeneous problem is solved using CPLEX (with the partitioning) in 224 seconds. While there is only less than $25 \%$ increase in the total number of variables and constraints due to the non-homogeneous fleet assumption, the result MIP problem cannot be solved even after 2 hours by CPLEX (with the partitioning).

A second experiment was conducted in order to study the impact of nonhomogeneous fleet on the service quality. In this numerical experiment each 10-car truck is replaced with 2 smaller 3-car and 7-car trucks. Empty and loaded truck cost factors are adjusted for smaller trucks and the SP-3 problem is solved using CPLEX. The results are presented in Figure 86. When the carrier utilizes smaller trucks the number of loaded/empty truck movements will be more compare to the homogeneous fleet case with 10-car trucks, therefore there is an increase in routing costs. However the average fill rate will be higher, more shipments are delivered and customers' associated costs will be less. 


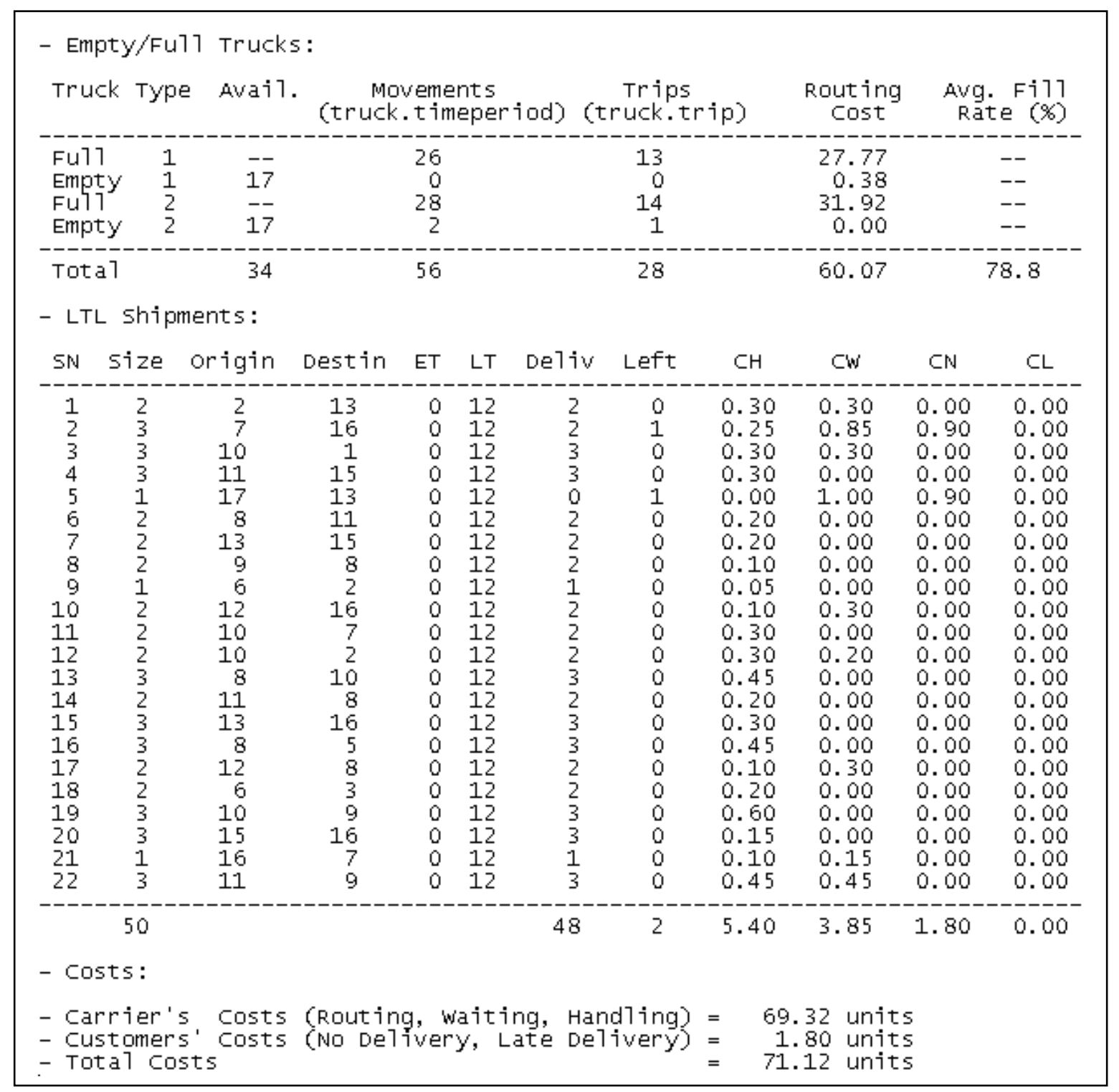

Figure 86 - Fleet information (Non-homogeneous fleet) 


\section{$\underline{\text { Simulation Experiment }}$}

All numerical experiments that have been reported in the previous sections the deal with a single snap shot of the operation. In all those examples it is assumed that at some point during the process, the demand and the supply information are known. The optimization problem is generated and solved to minimize the overall carrier/customer associated costs while the company is handling the shipment requests until the end of the planning horizon.

In the real world operations more and more requests for shipment delivery arise over time and the configuration of demand is changing. Therefore the optimizations must be conducted repeatedly on a rolling horizon setting to be used in the proposed decision making procedure. This section introduces an experiment that simulates a 10-day operation in order to evaluate the effectiveness of the proposed decision making method.

The auto carrier company operates on a 17-terminal network similar to the previous experiments. It is assumed that at the beginning of the simulation process there are 34 auto carrier trucks available over the region, two trucks at each hub/terminal. The fleet is homogeneous and all characteristics of the trucks are the same. The length of each time period in the time-space network is assumed to be equal to 6 hours. The shipment requests are randomly generated based on a homogeneous time Poisson process. The arrival rate is 10 shipments per time period (40 shipments per day). Origin and destination of loads are uniformly distributed over the 17-terminal network.

The simulation experiment is conducted based on the framework of the decision making procedure that is described in Chapter 4 (Figure 14). At the end of each time period or each time a new request arrives, all the demand/supply information is updated. Then the mathematical formulation of the problem is generated considering the updated empty truck locations, and the new shipments information.

After solving the problem, the performance of the new solution is analyzed and the effects of accepting the new requests are examined based on the acceptance 
criteria. Based on the acceptance criteria, the decision is being made either to accept the load or to reject it and the operation plan is prepared and sent to the field. The primary goal of conducting this simulation experiment is to evaluate the performance of the optimization approach using the literature-reported operation as the benchmark. Therefore, similar to the literature-reported operation all shipments are accepted for delivery.

Tables 26 and 27 show the simulation results using CPLEX (with SP-3) and the literature-reported operation, respectively. 40-time period (10-day) trucking operation has been simulated and the performance measures have been reported in these tables. Columns 2 and 3 show the number of shipments request at the beginning of each time period and the cumulative number of request arrivals. The total number of shipments that are in the system at each time period is reported on column 4. The average number of cars that are in-delivery at each moment throughout the simulation period is around 70 cars. Columns 2 and 3 show the number of shipments that are delivered at each time period and the cumulative number of deliveries. The last 4 columns contain the information on resource utilization in terms of the number of trips by empty and loaded trucks.

Figure 87 compares the two different planning approaches in terms of the number of cars that have been delivered. During the 10-day simulation period none of those approaches dominate the other. The literature-reported operation's delivery curve appears on top for most of the time, but the optimization method catches up, eventually. At the end of simulation the difference between the numbers of deliveries is less than $3 \%$.

Figure 87 shows that out of the total 400 cars that are accepted for delivery 319 cars are delivered using optimization method and 327 cars are delivered using literature-reported operation. However, as shown in Figure 88, the optimization approach has used the available resources more efficiently. During the last 5 days, where the operation reaches a steady state, 170 loaded trucks are dispatched using the literature-reported operation approach, while the optimization method handles the demand using only 90 loaded truck dispatches. 
Table 26 - Simulation Results (CPLEX with SP3)

\begin{tabular}{|c|c|c|c|c|c|c|c|c|c|}
\hline $\begin{array}{l}\text { Time } \\
\text { Period }\end{array}$ & $\begin{array}{c}\text { \# of } \\
\text { New } \\
\text { Shipments }\end{array}$ & $\begin{array}{c}\text { Total } \\
\text { \# of } \\
\text { Requests } \\
\text { Received }\end{array}$ & $\begin{array}{c}\text { \#of } \\
\text { Shipments } \\
\text { In } \\
\text { Delivery } \\
\end{array}$ & $\begin{array}{c}\text { \# of } \\
\text { Shipments } \\
\text { Delivered }\end{array}$ & $\begin{array}{c}\text { Total } \\
\text { \#of } \\
\text { Shipments } \\
\text { Delivered }\end{array}$ & $\begin{array}{c}\# \text { of } \\
\text { Empty } \\
\text { Truck } \\
\text { Trips } \\
\end{array}$ & $\begin{array}{c}\text { \# of } \\
\text { Loaded } \\
\text { Truck } \\
\text { Trips } \\
\end{array}$ & $\begin{array}{c}\text { Total } \\
\text { \# of } \\
\text { Truck } \\
\text { Trips } \\
\end{array}$ & $\begin{array}{c}\text { Avg. } \\
\text { \# of } \\
\text { Truck } \\
\text { Trips }\end{array}$ \\
\hline 0 & 0 & 0 & 0 & 0 & 0 & 0 & 0 & 0 & 0.0 \\
\hline 1 & 10 & 10 & 10 & 0 & 0 & 0 & 2 & 2 & 2.0 \\
\hline 2 & 9 & 19 & 16 & 3 & 3 & 0 & 1 & 3 & 1.5 \\
\hline 3 & 9 & 28 & 25 & 0 & 3 & 0 & 5 & 8 & 2.7 \\
\hline 4 & 11 & 39 & 36 & 0 & 3 & 0 & 5 & 13 & 3.3 \\
\hline 5 & 11 & 50 & 47 & 0 & 3 & 0 & 4 & 17 & 3.4 \\
\hline 6 & 9 & 59 & 54 & 2 & 5 & 0 & 7 & 24 & 4.0 \\
\hline 7 & 11 & 70 & 60 & 5 & 10 & 0 & 5 & 29 & 4.1 \\
\hline 8 & 9 & 79 & 63 & 6 & 16 & 0 & 3 & 32 & 4.0 \\
\hline 9 & 10 & 89 & 70 & 3 & 19 & 1 & 3 & 36 & 4.0 \\
\hline 10 & 10 & 99 & 77 & 3 & 22 & 0 & 5 & 41 & 4.1 \\
\hline 11 & 9 & 108 & 78 & 8 & 30 & 1 & 4 & 46 & 4.2 \\
\hline 12 & 12 & 120 & 83 & 7 & 37 & 0 & 4 & 50 & 4.2 \\
\hline 13 & 9 & 129 & 87 & 5 & 42 & 1 & 5 & 56 & 4.3 \\
\hline 14 & 9 & 138 & 94 & 2 & 44 & 1 & 4 & 61 & 4.4 \\
\hline 15 & 10 & 148 & 96 & 8 & 52 & 0 & 6 & 67 & 4.5 \\
\hline 16 & 12 & 160 & 88 & 20 & 72 & 1 & 5 & 73 & 4.6 \\
\hline 17 & 10 & 170 & 89 & 9 & 81 & 1 & 2 & 76 & 4.5 \\
\hline 18 & 9 & 179 & 95 & 3 & 84 & 0 & 7 & 83 & 4.6 \\
\hline 19 & 10 & 189 & 82 & 23 & 107 & 0 & 7 & 90 & 4.7 \\
\hline 20 & 11 & 200 & 67 & 26 & 133 & 0 & 5 & 95 & 4.8 \\
\hline 21 & 9 & 209 & 67 & 9 & 142 & 2 & 5 & 102 & 4.9 \\
\hline 22 & 11 & 220 & 67 & 11 & 153 & 0 & 6 & 108 & 4.9 \\
\hline 23 & 9 & 229 & 62 & 14 & 167 & 2 & 3 & 113 & 4.9 \\
\hline 24 & 10 & 239 & 67 & 5 & 172 & 0 & 4 & 117 & 4.9 \\
\hline 25 & 11 & 250 & 70 & 8 & 180 & 0 & 8 & 125 & 5.0 \\
\hline 26 & 9 & 259 & 69 & 10 & 190 & 0 & 2 & 127 & 4.9 \\
\hline 27 & 11 & 270 & 78 & 2 & 192 & 0 & 4 & 131 & 4.9 \\
\hline 28 & 10 & 280 & 81 & 7 & 199 & 2 & 7 & 140 & 5.0 \\
\hline 29 & 10 & 290 & 75 & 16 & 215 & 2 & 1 & 143 & 4.9 \\
\hline 30 & 9 & 299 & 79 & 5 & 220 & 1 & 5 & 149 & 5.0 \\
\hline 31 & 10 & 309 & 85 & 4 & 224 & 0 & 6 & 155 & 5.0 \\
\hline 32 & 11 & 320 & 88 & 8 & 232 & 0 & 4 & 159 & 5.0 \\
\hline 33 & 9 & 329 & 94 & 3 & 235 & 0 & 5 & 164 & 5.0 \\
\hline 34 & 10 & 339 & 85 & 19 & 254 & 1 & 5 & 170 & 5.0 \\
\hline 35 & 10 & 349 & 82 & 13 & 267 & 1 & 4 & 175 & 5.0 \\
\hline 36 & 11 & 360 & 82 & 11 & 278 & 0 & 6 & 181 & 5.0 \\
\hline 37 & 10 & 370 & 77 & 15 & 293 & 1 & 3 & 185 & 5.0 \\
\hline 38 & 10 & 380 & 80 & 7 & 300 & 0 & 4 & 189 & 5.0 \\
\hline 39 & 9 & 389 & 74 & 15 & 315 & 1 & 5 & 195 & 5.0 \\
\hline 40 & 11 & 400 & 81 & 4 & 319 & 1 & 3 & 199 & 5.0 \\
\hline Total & 400 & & & 319 & & 20 & 179 & & \\
\hline
\end{tabular}


Table 27 - Simulation Results (Literature-Reported Operation)

\begin{tabular}{|c|c|c|c|c|c|c|c|c|c|}
\hline $\begin{array}{l}\text { Time } \\
\text { Period }\end{array}$ & $\begin{array}{c}\text { \# of } \\
\text { New } \\
\text { Shipments }\end{array}$ & $\begin{array}{c}\text { Total } \\
\text { \# of } \\
\text { Requests } \\
\text { Received }\end{array}$ & $\begin{array}{c}\text { \#of } \\
\text { Shipments } \\
\text { In } \\
\text { Delivery } \\
\end{array}$ & $\begin{array}{c}\text { \# of } \\
\text { Shipments } \\
\text { Delivered }\end{array}$ & $\begin{array}{c}\text { Total } \\
\text { \#of } \\
\text { Shipments } \\
\text { Delivered }\end{array}$ & $\begin{array}{c}\text { \# of } \\
\text { Empty } \\
\text { Truck } \\
\text { Trips } \\
\end{array}$ & $\begin{array}{c}\text { \# of } \\
\text { Loaded } \\
\text { Truck } \\
\text { Trips }\end{array}$ & $\begin{array}{c}\text { Total } \\
\text { \# of } \\
\text { Truck } \\
\text { Trips }\end{array}$ & $\begin{array}{c}\text { Avg. } \\
\text { \# of } \\
\text { Truck } \\
\text { Trips }\end{array}$ \\
\hline 0 & 0 & 0 & 0 & 0 & 0 & 0 & 0 & 0 & 0.0 \\
\hline 1 & 10 & 10 & 10 & 0 & 0 & 0 & 3 & 3 & 3.0 \\
\hline 2 & 9 & 19 & 16 & 3 & 3 & 0 & 5 & 8 & 4.0 \\
\hline 3 & 9 & 28 & 22 & 3 & 6 & 0 & 3 & 11 & 3.7 \\
\hline 4 & 11 & 39 & 30 & 3 & 9 & 0 & 6 & 17 & 4.3 \\
\hline 5 & 11 & 50 & 38 & 3 & 12 & 0 & 6 & 23 & 4.6 \\
\hline 6 & 9 & 59 & 42 & 5 & 17 & 0 & 5 & 28 & 4.7 \\
\hline 7 & 11 & 70 & 51 & 2 & 19 & 0 & 8 & 36 & 5.1 \\
\hline 8 & 9 & 79 & 49 & 11 & 30 & 0 & 5 & 41 & 5.1 \\
\hline 9 & 10 & 89 & 50 & 9 & 39 & 0 & 5 & 46 & 5.1 \\
\hline 10 & 10 & 99 & 54 & 6 & 45 & 0 & 9 & 55 & 5.5 \\
\hline 11 & 9 & 108 & 54 & 9 & 54 & 0 & 10 & 65 & 5.9 \\
\hline 12 & 12 & 120 & 59 & 7 & 61 & 1 & 7 & 73 & 6.1 \\
\hline 13 & 9 & 129 & 58 & 10 & 71 & 1 & 6 & 80 & 6.2 \\
\hline 14 & 9 & 138 & 63 & 4 & 75 & 0 & 7 & 87 & 6.2 \\
\hline 15 & 10 & 148 & 67 & 6 & 81 & 0 & 10 & 97 & 6.5 \\
\hline 16 & 12 & 160 & 75 & 4 & 85 & 0 & 8 & 105 & 6.6 \\
\hline 17 & 10 & 170 & 80 & 5 & 90 & 2 & 8 & 115 & 6.8 \\
\hline 18 & 9 & 179 & 79 & 10 & 100 & 0 & 10 & 125 & 6.9 \\
\hline 19 & 10 & 189 & 79 & 10 & 110 & 0 & 12 & 137 & 7.2 \\
\hline 20 & 11 & 200 & 85 & 5 & 115 & 0 & 8 & 145 & 7.3 \\
\hline 21 & 9 & 209 & 83 & 11 & 126 & 0 & 10 & 155 & 7.4 \\
\hline 22 & 11 & 220 & 85 & 9 & 135 & 0 & 11 & 166 & 7.5 \\
\hline 23 & 9 & 229 & 82 & 12 & 147 & 0 & 6 & 172 & 7.5 \\
\hline 24 & 10 & 239 & 84 & 8 & 155 & 0 & 10 & 182 & 7.6 \\
\hline 25 & 11 & 250 & 80 & 15 & 170 & 2 & 8 & 192 & 7.7 \\
\hline 26 & 9 & 259 & 76 & 13 & 183 & 0 & 6 & 198 & 7.6 \\
\hline 27 & 11 & 270 & 78 & 9 & 192 & 0 & 12 & 210 & 7.8 \\
\hline 28 & 10 & 280 & 75 & 13 & 205 & 1 & 9 & 220 & 7.9 \\
\hline 29 & 10 & 290 & 69 & 16 & 221 & 0 & 7 & 227 & 7.8 \\
\hline 30 & 9 & 299 & 69 & 9 & 230 & 0 & 13 & 240 & 8.0 \\
\hline 31 & 10 & 309 & 72 & 7 & 237 & 0 & 7 & 247 & 8.0 \\
\hline 32 & 11 & 320 & 76 & 7 & 244 & 0 & 7 & 254 & 7.9 \\
\hline 33 & 9 & 329 & 76 & 9 & 253 & 0 & 11 & 265 & 8.0 \\
\hline 34 & 10 & 339 & 70 & 16 & 269 & 1 & 6 & 272 & 8.0 \\
\hline 35 & 10 & 349 & 72 & 8 & 277 & 0 & 8 & 280 & 8.0 \\
\hline 36 & 11 & 360 & 76 & 7 & 284 & 0 & 13 & 293 & 8.1 \\
\hline 37 & 10 & 370 & 77 & 9 & 293 & 0 & 5 & 298 & 8.1 \\
\hline 38 & 10 & 380 & 82 & 5 & 298 & 0 & 7 & 305 & 8.0 \\
\hline 39 & 9 & 389 & 80 & 11 & 309 & 0 & 9 & 314 & 8.1 \\
\hline 40 & 11 & 400 & 73 & 18 & 327 & 0 & 5 & 319 & 8.0 \\
\hline Total & 400 & & & 327 & & 8 & 311 & & \\
\hline
\end{tabular}




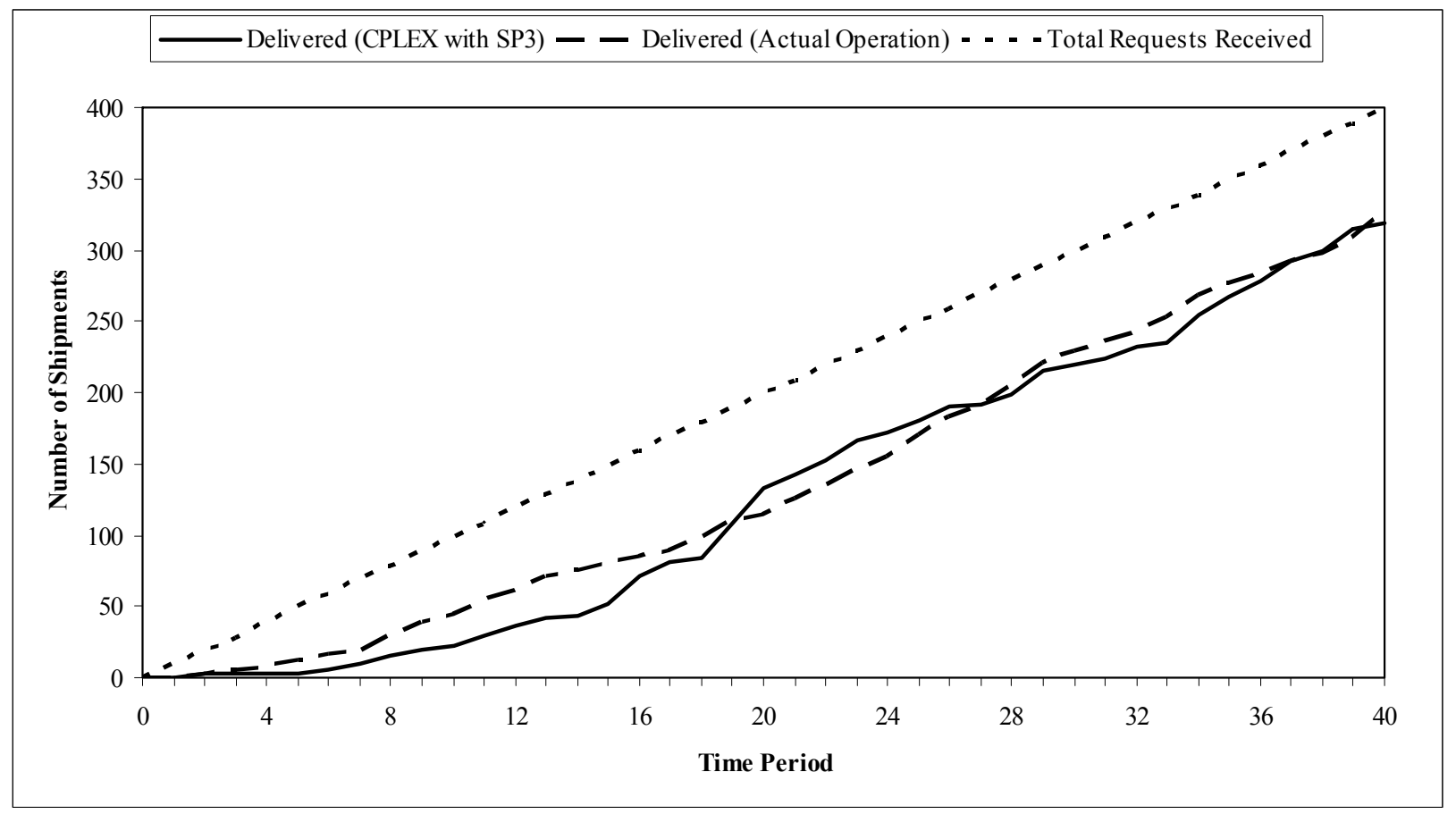

Figure 87 - Number of delivered shipments

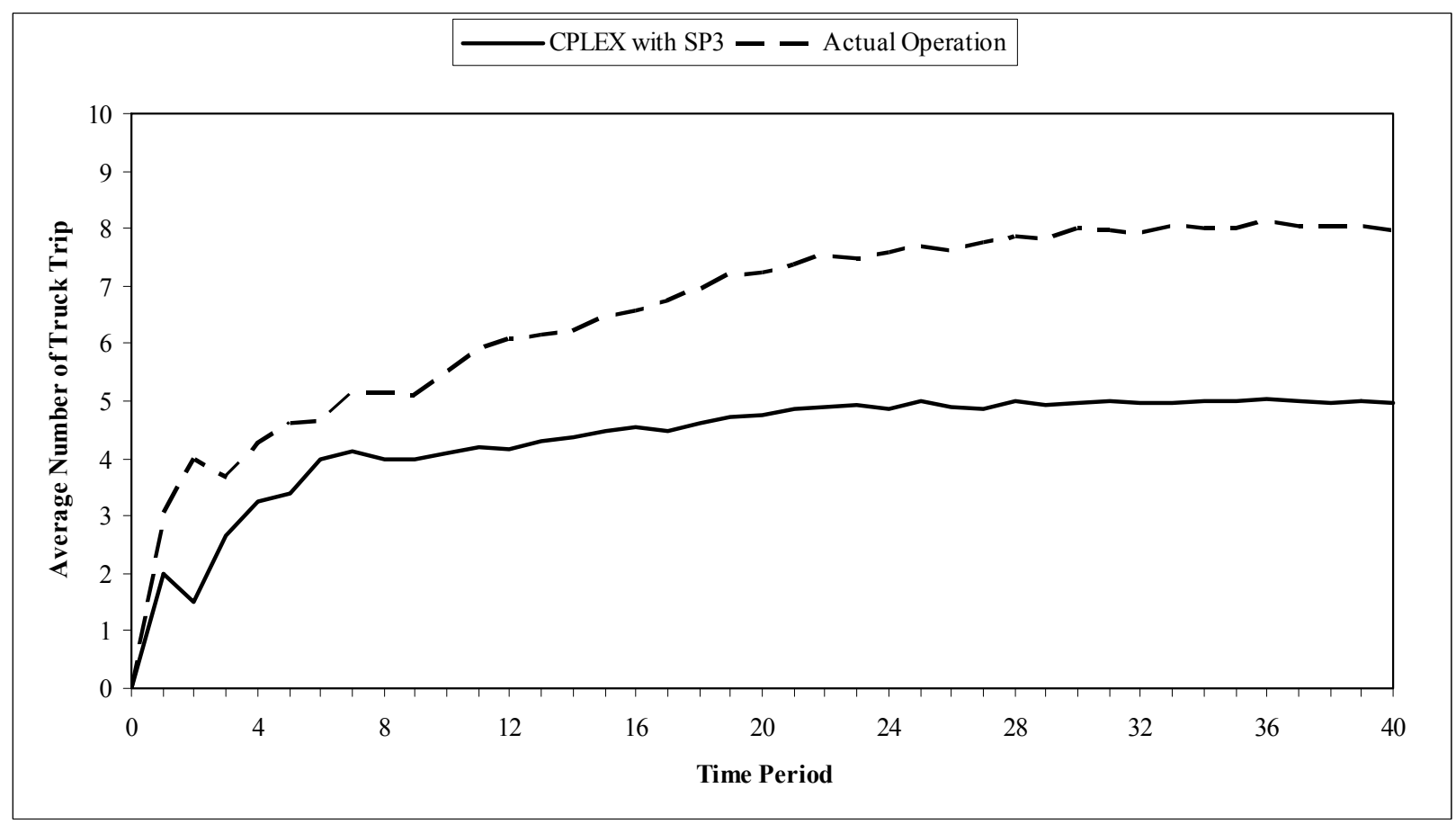

Figure 88 - Average number of truck trips 
In general the fuel efficiency of the auto carrier trucks is less than the regular tractor-trailers that carry containers or tankers. The reason is the large number of "open air" spaces (gaps) between the cars that cause greater disruption to the air flow and the resulting drag. The greater the drag, the more horsepower is required, which has a direct negative impact on the level of fuel consumption.

Considering 200 and 100 ton-mile/gallon gas consumption for empty and loaded trucks, and $\$ 4$ per gallon fuel cost, the total gas consumption of empty/loaded trucks on each 600 mile trip is equal to $\$ 240 / \$ 480$. Based on the above assumptions, the total fuel cost associated with optimization method and the literature-reported operation is equal to $\$ 82,560$ and $\$ 46,560$, respectively. Therefore, using the proposed optimization method would result more than $40 \%$ reduction in the operating costs.

Assuming that the company operates 360 days a year, the total annual savings in fuel costs is more than $\$ 2.5$ million if the company uses the optimization method for shipment routing and truck dispatching. Note that the annual revenue of this company is less than $\$ 10$, considering 40 shipment requests/day and $\$ 600$ revenue/shipment assumptions.

Figure 89 and 90 show the number of trucks across the network at each time period using a 3D surface. Both the top and the 3D views of the diagram are provided for both planning approaches. When the company uses the optimization method the trucks are concentrating at the center of the region (i.e. hubs 6, 7 and 12), as opposed to the literature-reported operation plans, based on which the trucks mainly stay at one side of the network (i.e. hubs 1-3 and 5-8). The observation shows that the optimization approach has better reaction to the demand pattern. Obviously, when the trucks are located at the central terminals better opportunity for consolidation would become available to the future demand, considering the fact that the origin and destination of requests are uniformly distributed.

The trucking company can use truck location 3D diagrams to make some decisions in strategic and tactical levels of planning to adjust the supply characteristics based on the demand pattern. 

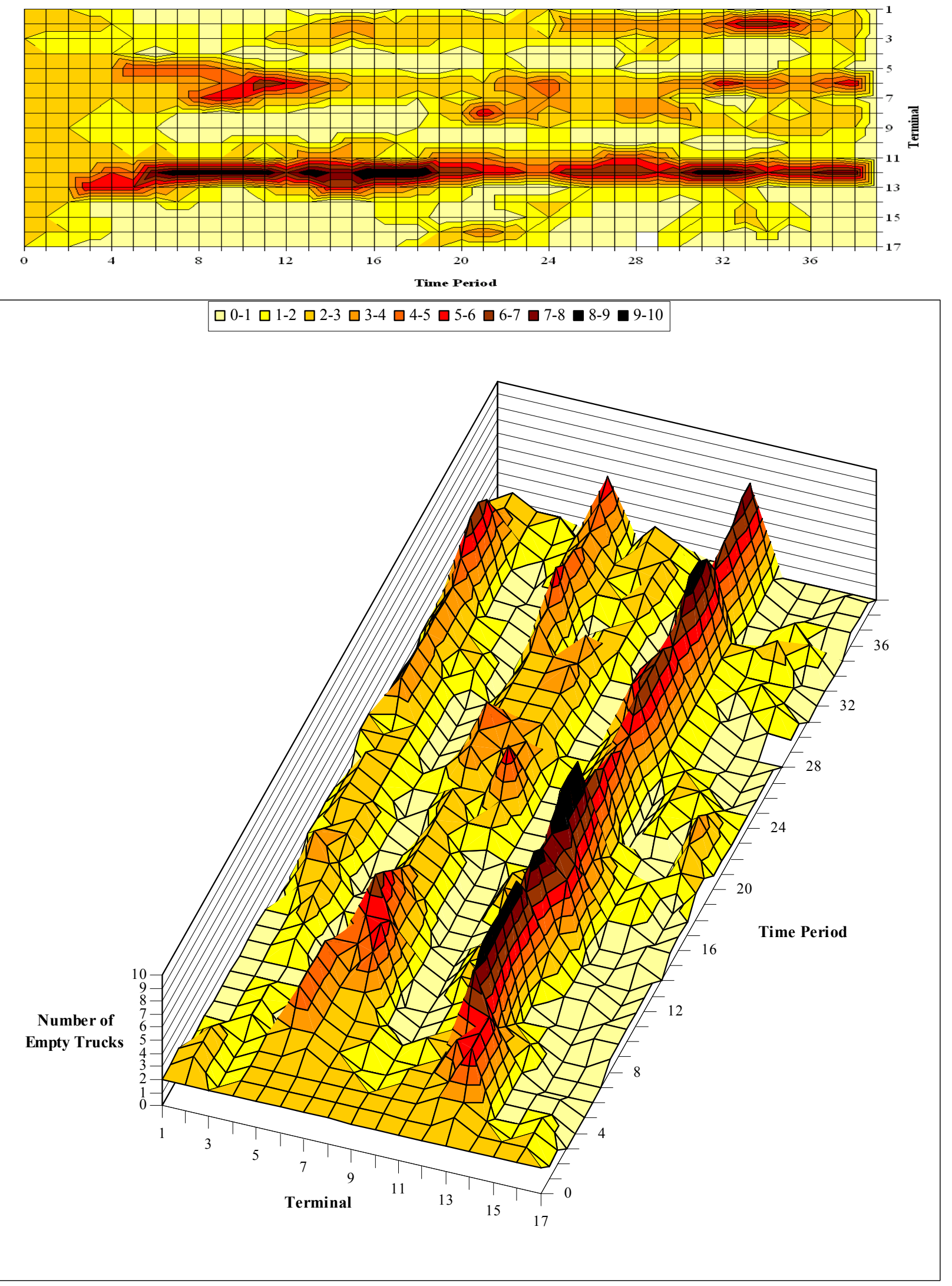

Figure 89 - Number of empty trucks at each terminal (CPLEX with SP-3) 

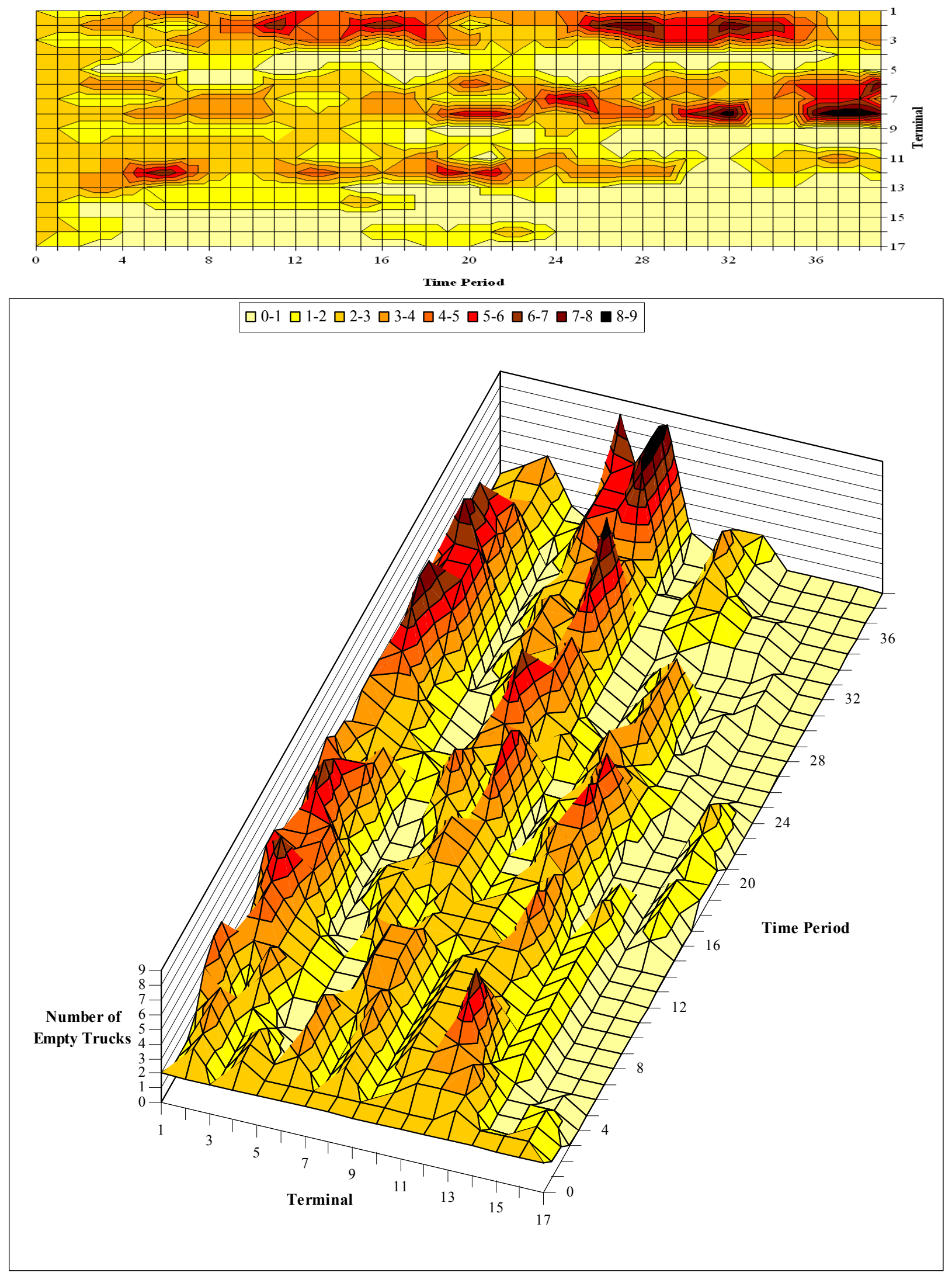

Figure 90 - Number of empty trucks at each terminal (literature-reported operation) 
Figures 91 and 92 present 2 other types of diagrams that show the performance of the decision making procedure. Figure 91 shows the waiting time of the first 250 shipment requests that are received and handled using the optimization approach. There are 4 set of shipments, shown with a circle on the diagram, with more than 3 days waiting time until their final delivery. Looking at the individual optimization results for the very first time period right after each shipment arrival, it appears that these shipments are among the 5-10\% that have not been delivered within the optimization horizon.

The results indicate that the dispatcher can rely on the optimization problem that is solved at each step as a tool to make the acceptance/rejection decisions. For instance, one criterion would be to reject a shipment if it cannot be delivered in two consecutive optimization runs.

Figure 92 shows the average cumulative waiting times for the first 250 shipments when the optimization method is used for decision making. The curve that appears on top is related to the case where all shipments are accepted for delivery. There are 23 shipments that waited for 2 or more days along the road before they reach their final destinations. If these 23 shipments get rejected, the waiting time curve would be similar to the one that appeared at the bottom on the diagram.

In this Chapter the result of the numerical experiments were reported. The first set of numerical experiments was conducted on a 10-terminal network to check the quality of solution provided by the proposed algorithm to solve the MIP problem. In the second numerical experiment the performance of the proposed methods were analyzed using a more realistic large-size 17-terminal network. Besides, a simulation framework was introduced and the effectiveness of the proposed decision making procedure was evaluated. 


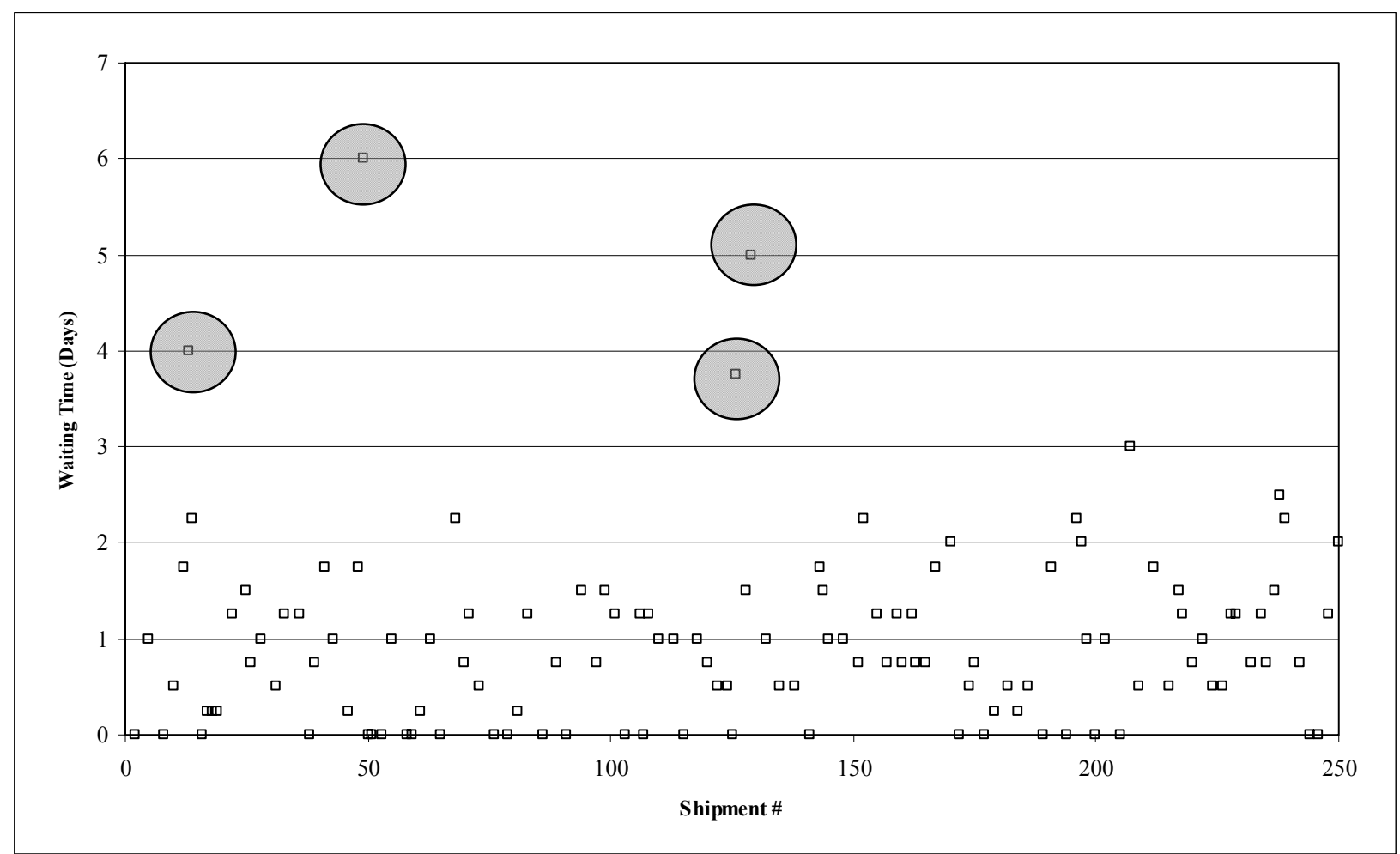

Figure 91 - Waiting time (CPLEX with SP-3)

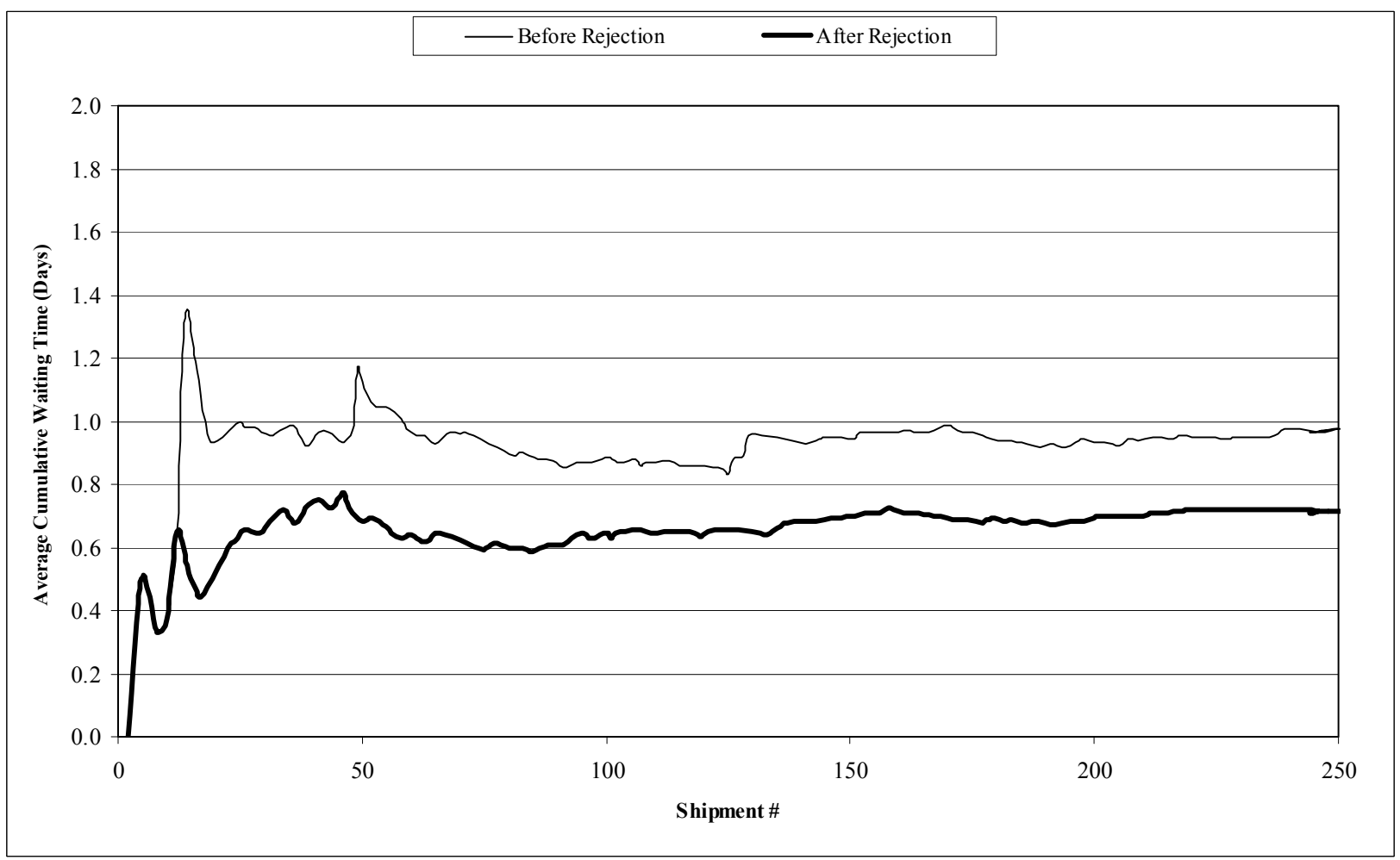

Figure 92 - Average cumulative waiting time (CPLEX with SP-3) 


\section{Chapter 8: Summary, Conclusions and Recommendations for Future Research}

The main contributions of this research were:

- To develop the a mixed integer programming (MIP) model to optimize the combined dynamic shipments routing and dynamic trucks routing and scheduling for LTL trucking operations

- To introduce a heuristic algorithm to solve the MIP problem

- To find a lower bound for the MIP problem, and check the quality of the solution provided by the heuristic algorithm

- To propose a decision making procedure to handle the requests for LTL shipments in an environment that changes dynamically

- To develop a set of simulation experiments to evaluate the effect of decision making techniques on LTL operations, and compare them to what the carriers do in practice (as the benchmark)

The dissertation started with an introduction of LTL trucking operations and different levels of planning for this type of motor carriers, followed by the review of literature that are related to tactical and operational planning. Following a brief discussion on MCNF problems and their solution algorithm, a mathematical model was proposed to deal with the combined shipment and routing problem.

A decision making procedure as well as a decision support application were presented. The main step in the decision making procedure was to solve the proposed mathematical problem. Three heuristic solution algorithms were proposed and the quality of the solutions was evaluated using a set of benchmark solutions.

Three levels of numerical experiments were conducted considering an auto carrier that operates on a hub-and-spoke network. The accuracy of the mathematical model and the behavior of the system under different demand/supply situations were 
examined. The performance of the proposed solution algorithms was compared and the Path-based portioning method was selected as the best solution method.

Fleet management products provide real-time, two-way data communication between trucks and dispatching centers, using which trucking companies move data as much as they move packages in order to improve their efficiency. This study proposed a decision making process that utilizes the data provided by the information network to improve productivity and customer service by optimizing the combined vehicle routing and shipment dispatching. This study suggested that a significant reduction in operational costs can be obtained as the result of using the proposed decision making process.

A list of suggestions and recommendation for the future research is as follows. These recommendations are divided into two groups. The first set includes those works that can be done based on the framework that is proposed in this study. These are research goals that can be achieved in short term to enhance the current system. The second part contains research recommendations that go beyond the scope of this study. Those studies are mainly time consuming and each can be a major research effort.

- Short-term studies:

Currently, some parts of the data handling efforts needed for decision making procedure are performed manually. Every time a new mathematical formulation is generated the resulting problem file is read and solved through the CPLEX dialog window. Then the solution is transferred from CPLEX $\log$ to a data file that is used by "Operation Plan" and "Data Update" C programs. One suggestion would be to used either the CPLEX concert technology or callable library and incorporate the CPLEX into the "Problem Generator" program or decision making application to facilitate the process of solving the MIP problem in a dynamic environment or simulation setting.

In Chapter 4 of this dissertation, 2 versions of the fast acceptance technique were proposed for decision making under high demand condition. These methods would increase the ability of handling the requests when solving the optimization 
problem is computationally expensive. A recommended future research would examine the performance of these techniques using the simulation framework that was introduced in the last Chapter of this dissertation.

The other direction for the future research would be to design additional acceptance/rejection criteria and decision making procedures and examine the effectiveness of these methods.

The heuristic search algorithm that is proposed in Chapter 6 of the dissertation is a locally optimal solution, which is not necessarily a "good" solution. One way to improve the solution quality is to apply the meta-heuristic methods incorporated into the proposed path-based search algorithm. In this study the simulated annealing procedure is used with the heuristic search algorithm, but the results were not promising. Future research efforts may focus on variations of the simulated annealing method or using other meta-heuristic methods (e.g. Genetic Algorithm or Tabu Search) to improve the quality of the solution provided by the path-based heuristic search.

In this study the "Operation Plan" program has been developed to interpret the solution of the mathematical problem and to generate the required operation plans. However, when the optimization is performed repeatedly the shipment and truck routing and scheduling plans are modified. A program is required to generate the overall operation plans based on the individual optimization results. Such a program can be used extensively to study the simulation results.

The proposed decision making procedure works based on the repeated optimization runs. A very crucial question is whether the mathematical problem must be solved at the end of each time period or a longer interval must be selected. Every time the operations plan changes the shipments that are already in the system need to adapt with the new plan. With longer intervals, there is more time to apply the previous routing plans before the new requests change the current operation plan. However, new shipments experience delay in delivery. Number of shipments would be more at each optimization run and this would increase the execution time. 
- Long-term studies:

An important dimension of the LTL trucking operations is driver management. Chapter 3 of this dissertation introduces the required additions to assumptions, notations, objective function and constraints of the original mathematical formulation in order to model the combined driver, truck and shipment routing and scheduling problem. Solving the resulting problem would be a direction for the future research.

In Chapter 3 of the dissertation, a new waiting cost element was introduced to deal with the partial delivery situation. Additional modifications applied to the latedelivery cost, waiting cost and shipment related constraints at shipment destination. The recommended future research effort would consider solving the resulting nonlinear optimization problem.

When satellite terminals are considered as a part of network, a more realistic problem is generated. A future study would deal with the local pickup and delivery problem in conjunction with the shipment and truck routing problem on the hub-andspoke network. The problem is non-homogeneous due to the fact that different types of trucks operate on different parts of the system.

The capacity of a hub and the efficiency of the hub operations have a significant impact on the performance of the trucking operations. Some previous research efforts focused on managing the hub operations (e.g. auto carrier's loading/unloading sequence) in order to reduce the associated operating costs. Another area for future research is to optimize the shipment routing and truck dispatching problem considering the hub operations.

The LTL trucking companies operate on intercity networks. However, most of the terminals (hubs) located in urban areas near the major metropolitans where congestions due to peak hour traffic, work-zones or accidents have a significant impact on the travel times and therefore the system performance. Imagine a truck that traveled over 600 miles from Charlotte to New York without any problem but cannot make the on-time delivery due to an accident that occurred within the last 5 miles of its trip. An idea for future study would be to consider travel time variability issue in the optimization model. 
Chapter 3 addressed the non-homogeneous fleet assumption by introducing a modified version of the proposed mathematical model. Chapter 6 discussed the impact of non-homogeneous fleet assumption on the complexity of the optimization model. A recommendation would be to study the impact of non-homogeneous fleet on the carrier decision making process, the carrier performance and also the customer satisfaction.

Considering the non-homogeneous fleet assumption, an important problem is to find the best fleet configuration. Another recommendation for future research is to consider solving this problem based on the demand pattern. This would help a company to figure out how many trucks, with what capacity must be either purchased or leased for each period of time. 


\section{References}

1. U.S. Department of Transportation (USDOT), 2006, Freight in America, Research and Innovative Technology Administration, Bureau of Transportation Statistics, Washington D.C.

2. U.S. Department of Transportation (USDOT), 2007, National Transportation Statistics, Research and Innovative Technology Administration, Bureau of Transportation Statistics, Washington D.C.

3. The American Trucking Associations Foundation (ATA), 1996, 21 ${ }^{\text {st }}$ Century Trucking: Volume 2- Profiles of the Future, Alexandria, Virginia

4. $\quad$ http://news.moneycentral.msn.com/ticker/article. aspx $?$ Feed=AP\&Date $=200$ 71029\&ID=7714102\&Symbol=US:SAIA

5. http://news.van.fedex.com/node/6585

6. Bureau of Transportation Statistics (BTS), Motor Carrier Financial and Operational Statistics, TranStats: http://transtats.bts.gov/

7. Hejazi, B. and Haghani, A., 2008, dynamic Decision Making for Less-ThanTruckload Trucking Operations, Transportation Research Record, 2032, 17 25

8. Regan A.C., H.S. Mahmassani, and P. Jaillet, 1995, Improving the Efficiency of commercial Vehicle Operations Using Real-Time Information: Potential Uses and Assignment strategies, Transportation Research Record, 1493, 188-198

9. Regan A.C., H.S. Mahmassani, and P. Jaillet, 1996, Dynamic Decision Making for Commercial Fleet Operations Using Real-Time Information, Transportation Research Record, 1537, 91-97

10. Regan A.C., H.S. Mahmassani, and P. Jaillet, 1998, Evaluation of Dynamic Fleet Management Systems: Simulation Framework, Transportation Research Record, 1645, 176-184

11. Yang J., P. Jaillet, and H.S. Mahmassani, 1998, On-line Algorithms for Truck Fleet Assignment and Scheduling under Real-time Information, Transportation Research Record, 1667, 107-11

12. Yang J., P. Jaillet, and H.S. Mahmassani, 2004, Real-time Multi-Vehicle Truckload Pick-up and Delivery Problem, Transportation Science, Vol. 38, No. 2, 135-148 
13. Jung S., 2000, A Genetic Algorithm for the Vehicle Routing Problem with Time-Dependant Travel Times, PhD Thesis, Department of Civil and Environmental Engineering, University of Maryland, College Park, Maryland

14. Mahmassani H.S., Y. Kim, and P. Jaillet, 2000, Local Optimization Approaches to Solve Dynamic Commercial Fleet Management Problems, Transportation Research Record, 1733, 71-79

15. Gendreau M., P. Badeau, F. Guertin, J.Y. Potvin, and E.D. Taillard, 1996, A Solution Procedure for Real-Time Routing and Dispatching of Commercial Vehicles, In $3^{\text {rd }}$ Annual World Congress on Intelligent Transportation Systems, Distributed on CD

16. Gendreau F. Guertin, J.Y. Potvin, and R. Seguin, 1998, Neighborhood Search heuristics for a Dynamic Vehicle Dispatching Problem with Pick-ups and Deliveries, Publication CRT-98-10, Center for Research on Transportation, University of Montreal

17. Gendreau M., F. Guertin, J.Y. Potvin, and E.D. Taillard, 1999, Tabu Search for Real-Time Vehicle Routing and Dispatching, Transportation Science, Vol. 33, No. 4, 381-390

18. Kim Y., H.S. Mahmassani, and P. Jaillet, 2002, Dynamic truckload Truck Routing and Scheduling in Over-Saturated Demand Situation, Transportation Research Record, 1783, 66-71

19. Kim Y., H.S. Mahmassani, P. Jaillet, 2004, Dynamic Truckload Routing, Scheduling, and Load Acceptance for Large Fleet Operation with Priority Demand, Presented at the $83^{\text {rd }}$ Annual Meeting of the Transportation Research Board

20. $\quad$ http://www.badbusinessbureau.com or http://www.ripoffreport.com

21. Delmore L., J. Roy, and J.M. Rousseau, 1988, Motor Carrier Operations Planning Models: A State of the Art, In Freight Planning and Logistics, Springer-Verlag, Berlin

22. Golden B.L. and A.A. Assad, 1988, Vehicle Routing: Methods and Studies, North Holland, Amsterdam

23. Crainic T.G. and G. Laporte, 1997, Planning Models for Freight Transportation, European Journal of Operational Research, Vol. 97, No. 3, $409-438$ 
24. Crainic T.G. and G. Laporte, 1998, Fleet Management and Logistics, Center for Research on Transportation, University of Montreal, Kluwer Academic Publisher, Boston

25. Roy J., 2001, Recent Trends in Logistics and the Need for Real-Time Decision Tolls in the Trucking Industry, Proceeding of the $34^{\text {th }}$ Hawaii International Conference on System Sciences

26. Powell W.B. and Y. Sheffi, 1983, The Load Planning Problem of Motor Carriers: Problem Description and a Proposed Solution Approach, Transportation Research, Vol. 17A, 471-480

27. Powell W.B. and Y. Sheffi, 1989, Design and Implementation of an Interactive Optimization System for the Network Design in Motor Carrier Industry, Operations Research, Vol. 37, 12-29

28. Powell W.B., 1986, A Local Improvement Heuristic for the Design of LTL Motor Carrier Networks, Transportation Science, Vol. 20, 246-257

29. Powell W.B., and Y.A. Koskosidis, 1992, Shipment Routing Algorithm with Tree Constraints, Transportation Science, Vol. 26, No.3, 230-245

30. Crainic T.G. and J. Roy, 1992, Design of Regular Intercity Driver Routes for the LTL Motor Carrier Industry, Transportation Science, Vol. 26, 280295

31. Roy L. and L. Delmore, 1989, NETPLAN: A Network Optimization Model for Tactical Planning in the Less-Than-Truckload Motor Carrier Industry, INFOR, Vol.27, No.1, 22-35

32. Crainic G. and J.M. Rousseau, 1986, Multicommodity, Multimode Freight Transportation: A General Modeling and Algorithmic Framework for the Service Network Design Problem, Transportation Research, Vol. 20B, 225242

33. Daganzo C., 1987, The Break-bulk Role of Terminals in Many-to-many Logistic Networks, Operations Research, Vol. 35, 543-555

34. Hall R.W., 1987, Comparison of Strategies for Routing Shipments through Transportation Terminals, Transportation Research, Vol. 21A, 421-429

35. Akyilmaz M.O., 1994, An Algorithmic Framework for Routing LTL Shipments, Journal of Operations Research Society, Vol. 45, 529-538 
36. Desrosiers J., M. Solomon, and F. Soumis, 1995, Time-Constrained Routing and Scheduling, Handbook in Operations Research and Management Science, Volume 8, Elsevier, Amsterdam

37. Assad A.A., 1978, Multicommodity Network Flows-A Survey, Networks, Vol.8, 37-91

38. Kennington J.L., 1978, A Survey of Linear Cost Multicommodity Network Flows, Operations Research, Vol.26, 209-236

39. Haghani, A.E., 1989, Formulation and Solution of Combined Train Routing and Makeup, and Empty Car Distribution Model, Transportation Research B: Methodological, Vol. 23B, No. 6, 433-452

40. Barnhart C. and Y. Sheffi, 1993, A Network-Based Primal-Dual Heuristic for the Solution of Multicommodity Network Flow Problem, Transportation Science, Vol. 27, 102-117

41. Farvolden J.M., W.B. Powell, and I.J. Lustig, 1993, A Primal Partitioning Solution for the Arc-Chain Formulation of a Multicommodity Network Flow Problem, Operations Research, Vol. 41, No. 4, 669-692

42. Farvolden J.M. and W.B. Powell, 1994, Subgradients for Service Network Design, Transportation Science, Vol. 28, 256-272

43. Kleywegt A.J. and J.D. Papastavrou, 1998, Acceptance and Dispatching Policies for a Distribution Problem, Transportation Science, Vol. 32, No. 2, $127-141$

44. Cheung R.K. and B. Muralidharan, 1999, Impact of Dynamic Decision Making on Hub-and-Spoke Freight Transportation Networks, Annals of Operations Research, Vol. 87, 49-71

45. Cheung R.K. and B. Muralidharan, 2000, Dynamic Routing for Priority Shipment in LTL Service Networks, Transportation Science, Vol. 34, No .1

46. Wang, I-Lin, 2003, Shortest Path and Multicommodity Network Flows, PhD Thesis, School of Industrial and Systems Engineering, Georgia Institute of Technology

47. Even S., Itai E.S. and Shamir A., 1976, On the Complexity of Timetable and Multicommodity Flow Problems, SIAM Journal on Computing, Vol. 5, No. 4, 691-703

48. Karp R., 1975, On the Computational Complexity of Combinatorial Problems, Networks, Vol. 5, No. 1, 45-68 
49. Dantzig G. and Wolfe P., 1961, The Decomposition Algorithm for Linear Programs, Econometrica, Vol. 29, 767-778

50. Ford Jr. L. and Fulkerson D., 1958, A Suggested Computation for Maximal Multicommodity Network Flows, Management Science, Vol. 5, 97-101

51. Barnhart C., Johnson E., Hane C. and Sigismondi G., 1995, An Alternative Formulation and Solution Strategy for Multicommodity Network Flow Problems, Telecommunication Systems, Vol. 3, 239-258

52. McBride R. and Mamer J., 2001, Solving the Undirected Multicommodity Flow Problem Using a Shortest Path-based Pricing Algorithm, Networks, Vol. 38, 181-188

53. Lin F. and Yee J., 1992, A New Multiplier Adjustment Procedure for the Distributed Computation of Routing Assignments in Virtual Circuit Data Networks, ORSA Journal on Computing, Vol. 4, No. 3, 250-266

54. Shepherd B. and Zhang L., 2001, A Cycle Augmentation Algorithm for Minimum Cost Multicommodity Flows on a Ring, Discrete Applied Mathematics, Vol. 110, No. 2-3, 301-315

55. Carolan W., Hill J., Kennington J., Niemi S. and Wichmann S., 1990, An Empirical Evaluation of the Korbx Algorithm for Military Airlift Applications, Operations Research, Vol. 38, No. 2, 240-248

56. Assad A., 1980, Modeling of Rail Networks: Toward a Routing/Makeup Model, Transportation Research, Part B: Methodological, Vol. 14B, No. 12, 101-114

57. Crainic T., Ferland J.-A. and Rousseau J.-M., 1984, A Tactical Planning Model for Rail Freight Transportation, Transportation Science, Vol. 18, $165-184$

58. Kwon O., Martland C. and Sussman J., 1998, Routing and Scheduling Temporal and Heterogeneous Freight Car Traffic on Rail Networks, Transportation Research Part E: Logistics and Transportation Review, Vol. 34, 101-115

59. Bellmore M., Bennington G. and Lubore S., 1971, A Multi-Vehicle Tanker Scheduling Problem, Transportation Science, Vol. 5, 36-47

60. Jewell W., 1957, Warehousing and Distribution of a Seasonal Product, Naval Research Logistics Quarterly, Vol. 4, No. 4, 29-34 
61. D'Amours S., Montreuil B. and Soumis F., 1996, Price-based Planning and Scheduling of Multiproduct Orders in Symbiotic Manufacturing Networks, European Journal of Operational Research, Vol. 96, No. 1, 148-166

62. Carden R. and Cheng C.K., 1991, A Global Router Using an Efficient Approximate Multicommodity Multiterminal Flow Algorithm, Proceedings of the $28^{\text {th }}$ ACM/IEEE Design Automation Conference, California, 316-321

63. Sarrafzadeh M. and Wong C., 1996, An Introduction to VLSI Physical Design, McGraw Hill, New York

64. Raghavan P., 1992, Integer Programming in VLSI Design, Discrete Applied Mathematics, Vol. 40, 29-43

65. Albrecht C., 2001, Global Routing by New Approximation Algorithms for Multicommodity Flow, IEEE Transactions on Computer-Aided Design of Integrated Circuits and Systems, Vol. 20, 622-632

66. LeBlanc L., Morlok E. and Pierskalla W., 1975, An Efficient Approach to Solving the Road Network Equilibrium Traffic Assignment Problem, Transportation Research, Vol. 9, 309-318

67. Ferris M., Meeraus A. and Rutherford T., 1999, Computing Wardropian Equilibria in a Complementarity Framework, Optimization Methods and Software, Vol. 10, No. 5, 669-685

68. Sensen N., 2001, Lower Bounds and Exact Algorithms for the Graph Partitioning Problem Using Multicommodity Flows, Proceeding of the $9^{\text {th }}$ annual European symposium, Aarhus, Denmark, Springer, 391-403

69. Leighton F. and Rao S., 1988, An Approximate Max-Flow Min-Cut Theorem for Uniform Multicommodity Flow Problems with Applications to Approximation Algorithms, Proceeding of the $29^{\text {th }}$ annual IEEE Symposium on Foundations of Computer Science, 422-431

70. Klein P., Agrawal A., Ravi R. and Rao S., 1990, Approximation Through Multicommodity Flow, Proceedings of the 31st annual IEEE Symposium on Foundations of Computer Science, Vol. 2, 726-737

71. Klein P., Rao S., Agrawal A. and Ravi R., 1995, An Approximate MaxFlow Min-Cut Relation for Undirected Multicommodity Flow with Applications, Combinatorica, Vol. 15, No. 2, 187-202

72. Leighton T. and Rao S., 1999, Multicommodity Max-Flow Min-Cut Theorems and their Use in Designing Approximation Algorithms, Journal of the ACM, Vol. 46, 787-832 
73. Gouveia L., 1996, Multicommodity Flow Models for Spanning Trees with Hop Constraints, European Journal of Operational Research, Vol. 95, No. $1,178-190$

74. Maurras J.-F. and Vaxes Y., 1997, Multicommodity Network Flow with Jump Constraints, Discrete Mathematics, Vol. 165-166, 481-486

75. Hadjiat M., Maurras J.-F. and Vaxes Y., 2000, A Primal Partitioning Approach for Single and Non-Simultaneous Multicommodity Flow Problems, European Journal of Operational Research, Vol. 123, 382-393

76. Girard A. and Sanso B., 1998, Multicommodity Flow Models, Failure Propagation and Reliable Loss Network Design, IEEE/ACM Transactions on Networking, Vol. 6, 82-93

77. Gendron B., Crainic T. and Frangioni A., 1999, Multicommodity Capacitated Network Design, Telecommunications Network Planning, Kluwer Academic Publisher, Boston

78. Tomlin J., 1966, Minimum-Cost Multicommodity Network Flows, Operations Research, Vol. 14, No. 1, 45-51

79. Ahuja R., Magnanti T.L. and Orlin J.B., 1993, Network Flows: Theory, Algorithms and Applications, Prentice Hall, Englewood Cliffs, New Jersey

80. Alvelos F., and Valério de Carvalho J.M., 2000, Solving Multicommodity Flow Problems with Branch-and-Price, Technical report, Dept. Produção e Sistemas, Universidade do Minho, Braga, Portugal

81. Karmarkar, N., 1984, A New Polynomial-Time Algorithm for Linear Programming, Combinatorial, Vol. 4, No. 4, 373-395

82. http://www.allaboutautotransport.com

83. Dijkstra, E., 1959, A Note on Two Problems in Connection with Graphs, Numerische Mathematik, Vol. 1, 269-271

84. Azevedo J.A., Santos Costa M.E.O., Silvestre Madeira J. J. E. R. and Martins E. Q. V., 1993, An Algorithm for the Ranking of Shortest Paths, European Journal of Operational Research, Vol. 69, 97-106

85. Brander A.W. and Sinclair M.C., 1995, A Comparative Study of K-Shortest Path Algorithms, Proc. $11^{\text {th }}$ UK Performance Engineering Workshop for Computer and Telecommunications Systems 
86. Carraresi P. and Sodini C., 1983, A Binary Enumeration Tree to Find K Shortest Paths, Proceedings of the $7^{\text {th }}$ Symposium of Operations Research, $177-188$

87. Chong E.I., Maddila S.R. and Morley S. T., 1995, On Finding SingleSource Single-Destination K Shortest Paths, Proceedings of the 7th International Conference on Computing and Information

88. Consiglio A. and Pecorella A., 1995, Using Simulated Annealing to Solve the K-Shortest Path Problem, Proceedings of Conference Italian Association of Operations Research

89. Dreyfus S.E., 1969, An Appraisal of Some Shortest Path Algorithms, Operations Research, Vol. 17, 395-412

90. Fox B.L., 1975, K-th Shortest Paths and Applications to the probabilistic networks, ORSA/TIMS Joint National Meeting, Vol. 23

91. Horne G.J., 1980, Finding the K Least Cost Paths in an Acyclic Activity Network, Journal of Operational Research Society, Vol. 31, 443-448

92. Kumar N. and Ghosh R. K., 1994, Parallel Algorithm for Finding First K Shortest Paths, Computer Science and Informatics, Vol. 24, No. 3, 21-28

93. Law A.G. and Rezazadeh A., 1993, Computing the K-Shortest Paths Under Nonnegative Weighting, Proceedings of the $22^{\text {nd }}$ Manitoba Conference on Numerical Mathematics and Computing, 277-280

94. Lawler E.L., 1972, A Procedure for Computing the K Best Solutions to Discrete Optimization Problems and its Application to the Shortest Path Problem, Management Science, Vol. 18, 401-405

95. Martins E.Q.V., 1984, An Algorithm for Ranking Paths that may Contain Cycles, European Journal of Operational Research, Vol. 18, No. 1, 123130

96. Minieka E., 1975, The K-th Shortest Path Problem, ORSA/TIMS Joint National Meeting, Vol. 23

97. Minieka E. and Shier D. R., 1973, A Note on Algebra for the K Best Routes in a Network, Journal of Institute of Mathematics and Its Applications, Vol. $11,145-149$

98. Perko A., 1986, Implementation of Algorithms for K Shortest Loopless Paths, Networks, Vol. 16, 149-160 
99. Ruppert E., 1997, Finding the K Shortest Paths in Parallel, Proceedings of 14th Symposium on Theoretical Aspects of Computer Science

100. Shibuya T., 1995, Finding the K Shortest Paths by AI Search Techniques, Cooperative Research Reports in Modeling and Algorithms, Vol. 7, No. 77, 212-222

101. Shier D.R., 1976, Algorithms for Finding the K Shortest Paths in a Network. ORSA/TIMS Joint National Meeting

102. Shier D.R., 1976, Iterative Methods for Determining the K Shortest Paths in a Network, Networks, Vol. 6, No. 3, 205-229

103. Shier D.R., 1979, On Algorithms for Finding the K Shortest Paths in a Network, Networks, Vol. 9, No. 3, 195-214

104. Skicism C.C. and Golden B.L., 1989, Solving K-Shortest and Constrained Shortest Path Problems Efficiently, Annals of Operations Research, Vol. 20, Network Optimization and Applications, 249-282

105. Weigand M.M., 1976, A New Algorithm for the Solution of the K-th Best Route Problem, Computing, Vol. 16, 139-151

106. Yen J.Y., 1971, Finding the K Shortest Loopless Paths in a Network, Management, Science, Vol. 17, 712-716

107. Yen J.Y., 1972, Another Algorithm for Finding the K Shortest Loopless Network Paths, Proceedings of $41^{\text {st }}$ Meeting of Operations Research Society of America, Vol. 20

108. Metropolis, N., Rosenbluth, A. W., Rosenbluth, M. N., Teller, A. H. and Teller, E., 1953, Equation of State Calculation by Fast Computing Machines, Journal of Chemical Physics, Vol. 21, 1087-1091

109. Kirkpatrick, S., Gellat, C. D. and Vecchi, M. P., 1983, Optimization by Simulated Annealing, Science, Vol. 220, 671-680

110. Dowsland, K. A., 1993, Some Experiments with Simulated Annealing Techniques for Packing Problems, European Journal of Operational Research, 68, 389-399

111. Dowsland, K. A., 1995, Simulated Annealing. In: Reeves, C. R. (Ed.), Modern Heuristic Techniques for Combinatorial Problems, McGraw-Hill, 21-69 
112. Johnson, D. S., Aragon, C. R., McGeoch, L. A. and Schevon, C., 1989, Optimization by Simulated Annealing: An Experimental Evaluation; Part I, Graph Partitioning, Operations Research, Vol. 37, 865-892

113. Johnson, D. S., Aragon, C. R., McGeoch, L. A. and Schevon, C., 1991, Optimization by Simulated Annealing: An Experimental Evaluation; Part II, Graph Colouring and Number Partitioning, Operations Research, Vol. 39, 378-406

114. Ben-Ameur, W., 2004, Computing the Initial Temperature of Simulated Annealing, Computational Optimization and Applications, Vol. 29, 369-385 